\title{
Ixcatec ethnoecology: plant management and biocultural heritage in Oaxaca, Mexico
}

\author{
Selene Rangel-Landa ${ }^{1,2}$, Alejandro Casas ${ }^{1 *}$, Erandi Rivera-Lozoya ${ }^{3}$, Ignacio Torres-García ${ }^{1}$ \\ and Mariana Vallejo-Ramos ${ }^{3}$
}

\begin{abstract}
Background: Studying motives of plant management allows understanding processes that originated agriculture and current forms of traditional technology innovation. Our work analyses the role of native plants in the Ixcatec subsistence, management practices, native plants biocultural importance, and motivations influencing management decisions. Cultural and ecological importance and management complexity may differ among species according with their use value and availability. We hypothesized that decreasing risk in availability of resources underlies the main motives of management, but curiosity, aesthetic, and ethical values may also be determinant.
\end{abstract}

Methods: Role of plants in subsistence strategies, forms of use and management was documented through 130 semi-structured interviews and participant observation. Free listing interviews to 38 people were used to estimate the cognitive importance of species used as food, medicine, fuel, fodder, ornament and ceremonial. Species ecological importance was evaluated through sampling vegetation in 22 points. Principal Components Analysis were performed to explore the relation between management, cultural and ecological importance and estimating the biocultural importance of native species.

Results: We recorded 627 useful plant species, 589 of them native. Livelihood strategies of households rely on agriculture, livestock and multiple use of forest resources. At least 400 species are managed, some of them involving artificial selection. Management complexity is the main factor reflecting the biocultural importance of plant species, and the weight of ecological importance and cultural value varied among use types. Management strategies aim to ensure resources availability, to have them closer, to embellish human spaces or satisfying ethical principles.

Conclusion: Decisions about plants management are influenced by perception of risk to satisfy material needs, but immaterial principles are also important. Studying such relation is crucial for understanding past and present technological innovation processes and understand the complex process of developing biocultural legacy.

Keywords: Biocultural heritage, Domestication, Ethnoecology, Tehuacán-Cuicatlán Valley, Ixcatec, Cultural value, Plant management

\section{Background}

In most rural areas of Mexico, especially in those inhabited by indigenous peoples, human subsistence patterns generally involve multiple strategies. Agriculture for direct consumption of products is commonly the main activity, complemented by small scale livestock and the use of numerous forest resources destined to direct consumption

\footnotetext{
* Correspondence: acasas@cieco.unam.mx

${ }^{1}$ Instituto de Investigaciones en Ecosistemas y Sustentabilidad, UNAM, Antigua Carretera a Pátzcuaro 8711, Morelia, Michoacán 58190, Mexico Full list of author information is available at the end of the article
}

and commercialization [1]. These activities occur in territories that are settings of multidimensional and complex interrelationships between humans and nature in socioecological systems, integrated as totalities with elements and processes mutually influencing their features and changes [2]. Expressions of these interrelationships are management of wild plant and animal species, domesticated organisms and territories of indigenous and local peoples, which constitute part of the biocultural heritage that are created and maintained through long term by the continuous use and management [3-5]. Management or 
transformations and decisions made by humans on ecosystems, and on their elements and functions [6], based on TEK are fundamental in the biocultural heritage development process, and constitute a traditional form of facing the uncertainty inherent to complex systems [3, 7-9].

Management may include a broad spectrum of strategies and interactions for appropriation and maintaining natural resources $[6,10,11]$; collective actions to protect them [12], as well as those directed to recover or restore them [6]. These practices (praxis) are based on TEK about species and ecosystems (corpus) that are in turn strongly linked to beliefs systems (kosmos) [7, 13], which have direct influence on resources and ecosystem management.

Plant management is influenced by ecological and social factors [14-17], including the cultural importance of plant species in human life. Some investigations have found positive correlation between cultural and ecological importance, suggesting that most conspicuous plants have more important use values, but numerous examples have been reported contradicting this hypothesis $[18,19]$. More informative for constructing ethnobiological theory has been analyzing the complex of the relationships between cultural significance, ecological importance and management complexity. In edible plants, it has been found that species with high cultural value and limited availability are more intensely managed, as a response to the risk in their availability [14-17]. However, humans are not only respondents of critical situations. Curiosity, attraction for beauty, experimentation, innovation, among other intentions are part of human nature and should also be taken into account as factors influencing people's decision to manage organisms [20-22].

Understanding the role of plant resources with different use types in human subsistence patterns, how management interactions are, and how are these influenced by social and ecological factors, may help to understand the principles of the construction of management techniques, management systems, how processes of domestication are originated, and how processes of current technical innovations are developed, in order to understand the process of construction of the biocultural heritage [6].

The Tehuacán-Cuicatlán Valley in central Mexico, is an important region of the Mexican biocultural heritage [3], harbouring more than 3,000 species of vascular plant species and human cultures with ancestors nearly 10,000 years old [23, 24]. Currently, the Popolocan, Mazatec, Mixtec, Chinantec, Cuicatec, Ixcatec, Chocho, Náhuatl and Mestizo communities make use of nearly 1,750 plant species, at least 610 of them receiving management practices $[11,25]$. These figures make the Tehuacán Valley an ideal setting for studying processes influencing decision, innovation and diffusion of experiences on plant management.

This study was performed in Santa María Ixcatlán, the only town where the Ixcatec currently live in the world.
It was directed to document subsistence strategies, plants use and management locally practiced, and the main motives to manage them. Also, we examined how cultural, ecological and management factors interact and determine the importance of native plants with different use type on Ixcatec biocultural heritage.

We analyzed the hypothesis that the main motive of managing plants is decreasing the risk that represent their low availability and in some cases to enhance their abundance and quality. Therefore, subsistence is based on multiple activities, diversified management strategies to prevent risks in staple resources availability; and the high cultural importance and management intensity may be associated with low ecological importance. But, attraction for beauty, curiosity and ethical concerns, beyond the satisfaction of primary needs, should also be important aspects in decisions to manage plant resources.

\section{Methods \\ Study area}

At present, the Ixcatec live only in the community of Santa María Ixcatlán, a town governed by the regime of traditional practices and customs. Land tenure is communal with 41,530 ha $[26,27]$ belonging to the TehuacánCuicatlán Biosphere Reserve, Mexico (Fig. 1). The whole territory is mountainous, with elevations ranging from 800 to $2600 \mathrm{~m}$. Soils in most of the territory derived from calcareous rocks, with thin layers of black organic soils. The town has temperate climate, with annual mean temperature of $17.2{ }^{\circ} \mathrm{C}$, and annual rainfall averaging $721 \mathrm{~mm}[28,29]$. The rest of the territory has semiarid climate [29]. Vegetation types are oak forests, tropical dry forest, induced grassland and secondary vegetation [30].

In Santa María Ixcatlán live 175 households and 516 people [31]. There is a high migration of young people to the cities of Tehuacán, México, Orizaba, and more recently to the US [32]. Local households' economy is based on direct consumption of agricultural products, livestock raising and use of forest products [32, 33]. The Communitarian Assembly, conformed by all adult men, is the maximum authority [32], and people obtain rights to have access to resources and lands of the territory through a system of charges and cooperation to communitarian activities [32]. Practically all families are Catholic [32], and have a complex calendar of ceremonies [27, 32, 33]. Nearly a dozen of persons are fluently speakers of Ixcatec, an almost extinct language [34, 35].

\section{Flora inventory}

We conducted ethnoecological studies in Ixcatlán in the period 1999-2001 and in the period 2011-2015 with 16 campaigns of field work. Trial walks accompanied with local informants were carried out to identify vegetation types [36] and collecting botanical voucher specimens 


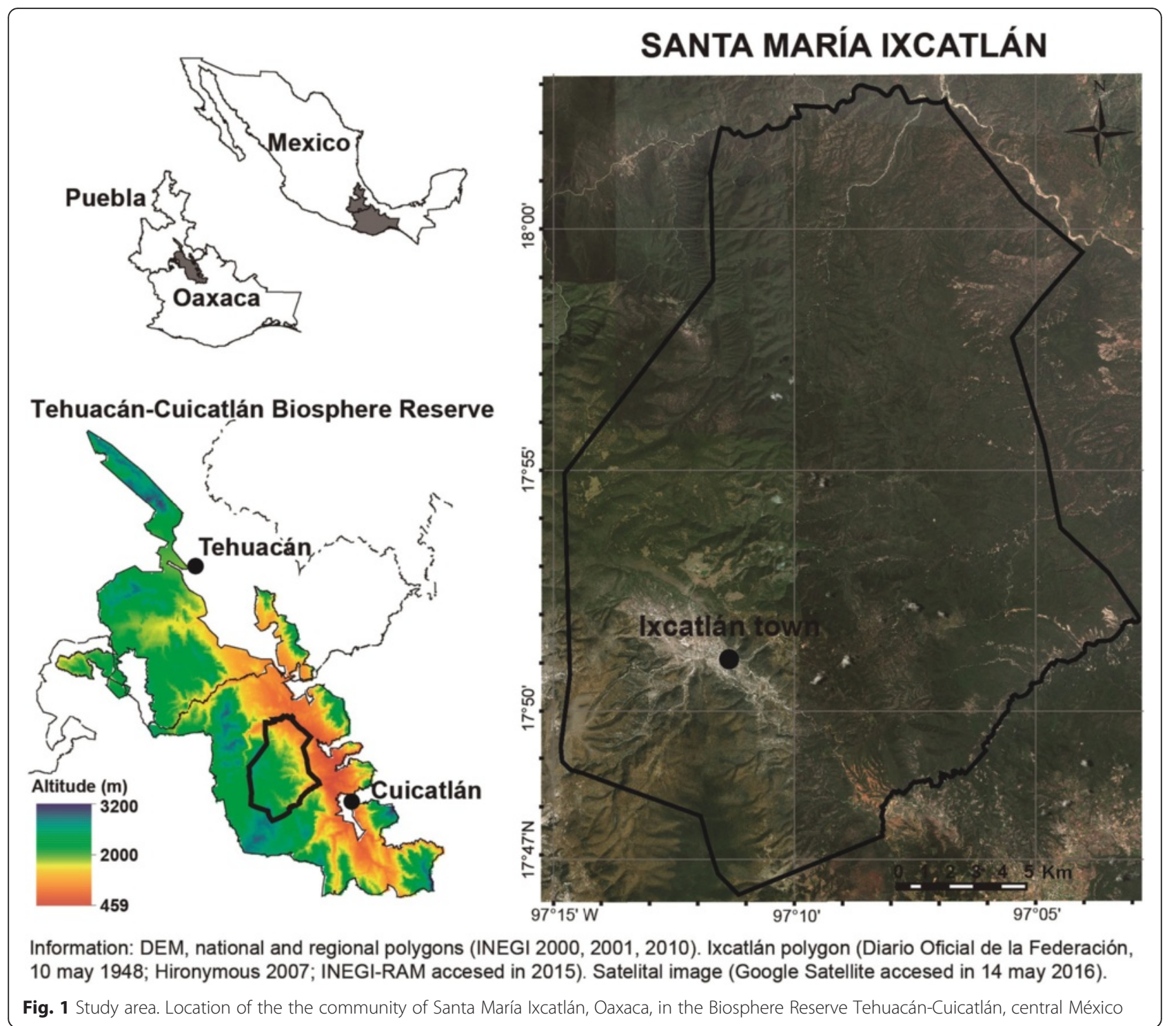

throughout the territory of the community. Voucher specimens were deposited at MEXU, EBUM, IE-BAJÍO and IBUG herbaria with Selene Rangel, Erandi Rivera, and Ricardo collection numbers. Nomenclature and classification of species are presented following the APG III classification system consulted in the site www.theplantlist.org [37].

\section{Interviews}

A total of 130 semi-structured interviews to 62 people were conducted to document common names of plants, their use, management practices and motivations to conduct them. Alive plants in their own homegardens, agricultural fields or seen in trial walks, fresh specimens collected a day before, dried specimens and pictures were used as stimulus in these interviews; 22 of the 62 interviewees ( 9 women and 13 men, with average age of 58.9 years, $\mathrm{SD}=22.5$ ) were considered key informants because of their deep knowledge of the territory and plants or because they were Ixcatec speakers. Key informants were selected by the snowball sampling technique, by asking for people with these skills; 15 of them were interviewed from 2 to 11 times in a total of 77 audio or video-recorded sessions, in which on average 17.2 $(\mathrm{SD}=23.4)$ species were reviewed per work session. The other 40 interviewees were considered occasional participants (21 female and 17 male, whose age averaged 53.2 years, $S D=20.8$ ), and they were selected randomly.

More detailed information about informants and activities are included in the Table 6 of Appendix. All interviews used for the analysis showed in this paper were performed in Spanish. All interviews and participant observation data about plant resources use and management were transcribed and systematized into the format of the ethnobotanical data base of Mexico (BADEPLAM) of the 
Botanical Garden, UNAM. Audio-visual material was stored in the Ixcatec Culture Archive and The Endangered Languages Archive.

\section{Surveys}

Semi-structured surveys with questions on agricultural production and consumption of plant resources were conducted in Spanish between 2000 and 2012 to 21 and 20 households representing the $12 \%$ of the households of Ixcatlán in each year (householders averaging 61.2 years old, SD = 17.2). In 2000 households were selected at random, while in 2012, $24 \%$ of the households surveyed in 2000 were selected, and the rest were selected at random.

\section{Free listing}

In order to identify the plant species with the higher cognitive importance, in 2013 we used the free listing method [38]. We requested in Spanish to 38 people (22 men and 16 women, aging on average 50.6 years, $\mathrm{SD}=$ 18.8) to spontaneously listing the names of plants that grow in the territory of Santa María Ixcatlán that are used: 1) as food, 2) to attend illnesses and take care of health, 3) as firewood, 4) to feed livestock, 5) to offer them to Saints, dead people or used in ceremonies, and 6) to embellish the houses and crop land. Once informants stopped listing plants for one use, we asked them to listing plants for other use, and we continued this procedure until finishing the lists of plants for the six uses. Of the 38 people interviewed, 19 were previous informants (13 considered key informants and 6 occasional informants), the other 19 people interviewed were selected at random. Details on the number of lists per use type, the number of items named, the levels of saturation of the datasets, and information about interviewees can be consulted in the Appendix.

\section{Vegetation sampling}

We conducted vegetation samplings in 22 points of nine natural and transformed vegetation types in order to estimate the ecological importance value of species [36]: Quercus liebmanni and Quercus laeta forest (3 points), Quercus urbanni forest (1 point), riparian forest of Taxodium huegelii (1 point), Juniperus flaccida forest (2 points), izotal of Beaucarnea stricta (2 points), mexical (2 points), palm scrubland of Brahea dulcis (2 points), grassland ( 2 points), and agricultural fields (7 points). At each point we established a $500 \mathrm{~m}^{2}$ quadrant, where all shrubs and trees were counted and their height and two canopy diameters were measured. Herbs were sampled in five subplots $\left(1 \mathrm{~m}^{2}\right.$ each) randomly placed within the area of each $500 \mathrm{~m}^{2}$ quadrant. Density and frequency was calculated for each species. Shrubs and trees biomass was calculated through volume formulas of geometric figures [39]. In addition, the floristic composition was sampled in 17 homegardens.

\section{Data analyses}

Livelihood analysis was conducted to assess the subsistence strategies [38], and descriptive data of use and management of plants species were estimated.

Series of Principal Component Analyses (PCA) with native plants species (species with wild populations or Mesoamerican species with naturalized populations in Ixcatlán territory), were performed. Species were considered as operational taxonomic units according to its number of uses, cognitive importance, consumption, ecological importance, complexity of management practices, and management place, all of them aspects involved in the definition of their importance to the biocultural heritage of plant species. The scores of the first principal component obtained in each PCA were considered as biocultural importance index by type of use, since these values are linear combinations that integrating information of the variables, species with positive and highest values were considered more important $[15,40]$. The most important variables and how they interact was identified by the correlation values between variables and the first two components [41]. We also identified how species are grouped according with all the variables studied by representing the cloud of species in terms of the two first components [41]. These PCAs were made in JMP 8. statistical software [42].

The cognitive importance was estimated through free listing data with the index of Sutrop $(S)$ with the formula $\mathrm{S}=\mathrm{F} /(\mathrm{N} \mathrm{mP})$, where $\mathrm{F}$ represents the frequency of the species, $\mathrm{N}$ the total number of interviewed people per use category, and $\mathrm{mP}$ is the medium position in which the term or species was named [43]. We calculated this index with the software FLAME v1.0 [44]. A zero value was assigned to all species that were not listed by consultants [43]. When an informant said that he/she does not know any plant for a given use or when he/she said that all plants could be used for the requested use, we excluded the list of the analysis.

The consumption of products was estimated as the percentage of households that consumed each plant species throughout the year, based on data documented with surveys conducted in 2012.

The ecological importance of species was estimated through the ecological importance value index EIVI $=($ Relative frequency + Relative abundance + Relative biomass $) / 3$, calculated by each plant species per sampled site [45]. The floristic composition of homegardens was similarly used to calculate ecological importance.

The complexity of management practices was calculated by the sum of numerical values of management practices. Values were assigned based on the typology proposed by Blancas et al. [11] as follows: a) gathering, simple or planned extraction strategies $=1 ; \mathrm{b}$ ) tolerance or let standing of plants $=2$; c) enhancement by promoting abundance of useful plant species or phenotypes $=3$; d) 
protection of desirable plants $=4$; e) transplanting entire individuals $=5 ; \mathrm{f}$ ) propagation as seed sowing and vegetative propagation $=6$. In addition, we assigned values of 0.5 to simple foraging by domestic animals, and uproot or deliberate removal individuals of the species in question. Values of each practices was summarized per plant species. The places of management were categorized in natural populations plants distribution sites (in situ $=1$ ) and sites out of their natural distribution $(e x$ situ $=2)[15,16]$.

\section{Results}

\section{Subsistence strategies}

Households are basic units making decisions on economic activities and forest resource management (Fig. 2). Agriculture is the main activity of all households, but maize and beans produced are insufficient to satisfy their annual requirements (Table 1). Multiple-cropping agriculture in the rainy season is carried out in terrains of 1 to 2 ha located around the town (95\% of households), and in homegardens ( 0.25 to 0.5 ha, managed by $30 \%$ of households) (Figs. 1, 3 and 4). Prayers and rituals drawing or putting crosses made with plants, offering alcoholic beverages to the earth, among other practices, are common during agricultural labours, seed selection and storage, sowing and harvest, as individual farmer or collective petitions for a good rainy season.

All people interviewed referred to difficulties in agriculture, mainly due to a low soil fertility and water scarcity. However, people deal with these problems in homegardens and agricultural fields by adding domestic animals manure, oak forest humus, ash, firewood debris and organic waste; agrochemicals are not used at all. In homegardens, recycling water and spatial arrangement of plants according with their water requirements are common. In agricultural fields, terraces and live fences are common for preventing soil erosion, as well as some dams for the accumulation of soil and moisture (Fig. 4).

Animal husbandry is practiced by almost all households as a saving for emergencies, animal power for agricultural and for gathering activities, only $5 \%$ of households commercialize animals in regional markets (Fig. 2). Nearly $55 \%$ of households raise animals in backyards (1-7 chickens, 1-9 turkeys or 1-4 pigs), $75 \%$ nurture draft animals (1-5 donkeys-mules or $1-4$ horses), and $25 \%$ raise livestock (5-80 cows, $10-16$ sheeps or $5-70$ goats) (Fig. 2). Animals feeding bases on domestic sub-products, maize straw, herbs managed in homegardens and agricultural fields, and foraging in communal lands (Figs. 3 and 4).

Gathering and management of native and introduced plants for direct consumption is practiced by all households (Figs. 2 and 3). Plants provide all the firewood and fodder needed and great part of food, medicines, materials for construction, tools, and other goods. Other important plants are ceremonial and ornamental, which are gathered and managed for direct use or as gifts to relatives (Fig. 2).

Few plant resources or their products are destined to economic interchange, the most important are Brahea dulcis and Agave potatorum (Fig. 2). The weaving of hats with Brahea dulcis leaves is carried out by nearly $84 \%$ of the households, while $10 \%$ are specialized in handcrafting baskets, covers for bottles and other products. Hats are interchanged almost every day for maize, food

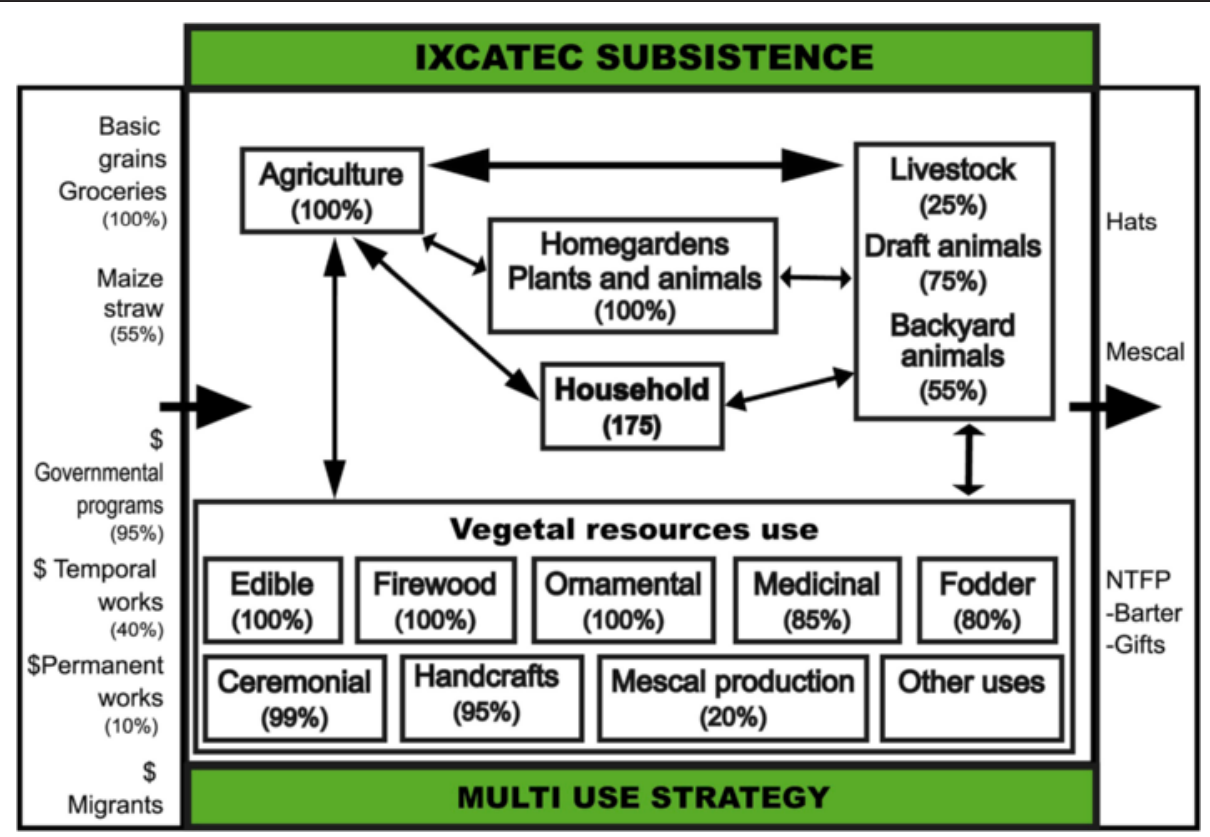

Fig. 2 General pattern of multiple use strategy of natural resources for subsistence in the community of Santa María Ixcatlán 
Table 1 Average and standard deviation of the amounts of maize and beans consumed, produced and productivity (kg/ha) achieved by people of Santa María Ixcatlán, Oaxaca for the periods of the years 1999-2000 and 2011-2012

\begin{tabular}{lllll}
\hline & Maize & & Bean \\
& $1999-2000$ & $2011-2012$ & $1999-2000$ & $2011-2012$ \\
\hline Consumption per year $(\mathrm{kg})$ & $766.38 \pm 94.34$ & $701.7 \pm 73.6$ & $155.6 \pm 19.4$ & $112.2 \pm 23$ \\
Production by household $(\mathrm{kg})$ & $285.5 \pm 79.9$ & $129.7 \pm 62.6$ & $76.2 \pm 26.9$ & $48 \pm 18.6$ \\
Productivity $(\mathrm{kg} / \mathrm{ha})$ & $289 \pm 70.5$ & $82.1 \pm 46.7$ & $43.9 \pm 10$ & $28.6 \pm 9.4$ \\
Community deficit $(\mathrm{T})$ & 82.7 & 100 & 13.7 & 11.2 \\
\hline
\end{tabular}

Data according to surveys realized to 21 households in 2000 and 20 households in 2012. Values are means and standard errors

or money in local stores. From 2011 to 2015 the price of each hat was 0.16 US dollars (based on an interchange rate of $\$ 20.00$ Mexican pesos by one American dollar), while in 2000 it was $\$ 0.12$. A household weave on average $28.9 \pm 3.65$ hats per week, and each hat requires 4.1 young leaves, which means approximately one million of leaves used in the whole community per year. Leaves extraction is carried out mainly in palm scrublands, where Brahea dulcis is promoted, protected and tolerated in areas of agricultural fields, but it is widely distributed throughout the whole territory (Figs. 3 and 4). For extracting palm leaves, people cut the young leaves without damaging the apical meristem and avoid gathering leaves during the new moon, otherwise they consider the growth of new leaves can be delayed. Harvesting palm leaves for direct use and local interchange is allowed but sale to regional sellers is forbidden. Palm is considered staple plant as people said "palms are our life because with palm leaves we make hats and we can get all we need to live".

Approximately $20 \%$ of households prepare mescal with Agave potatorum once to 10 times per year (4.8 \pm 1.49) (Fig. 2). For 2012 we estimated that the whole community produced 192 mescal batches, using $91.14 \pm$ 9.78 agaves per batch, in total nearly 17,500 agaves per year, whereas for the year 2000 we estimated the use of 4,900 individuals. The price of one litre of mescal was \$2.5 US dollars in 2000 and from $\$ 6$ to $\$ 9$ in 2011 to 2015. Although Agave potatorum is widely distributed in temperate and warm parts of the territory of the community (Figs. 3 and 4), the mescal producers said that they have to go progressively farer to extract agaves and they even complement their needs buying agaves to neighbouring communities; sometimes they complement their batches with the wild Agave vivipara extracted in the warm land of the territory. Agave extraction is

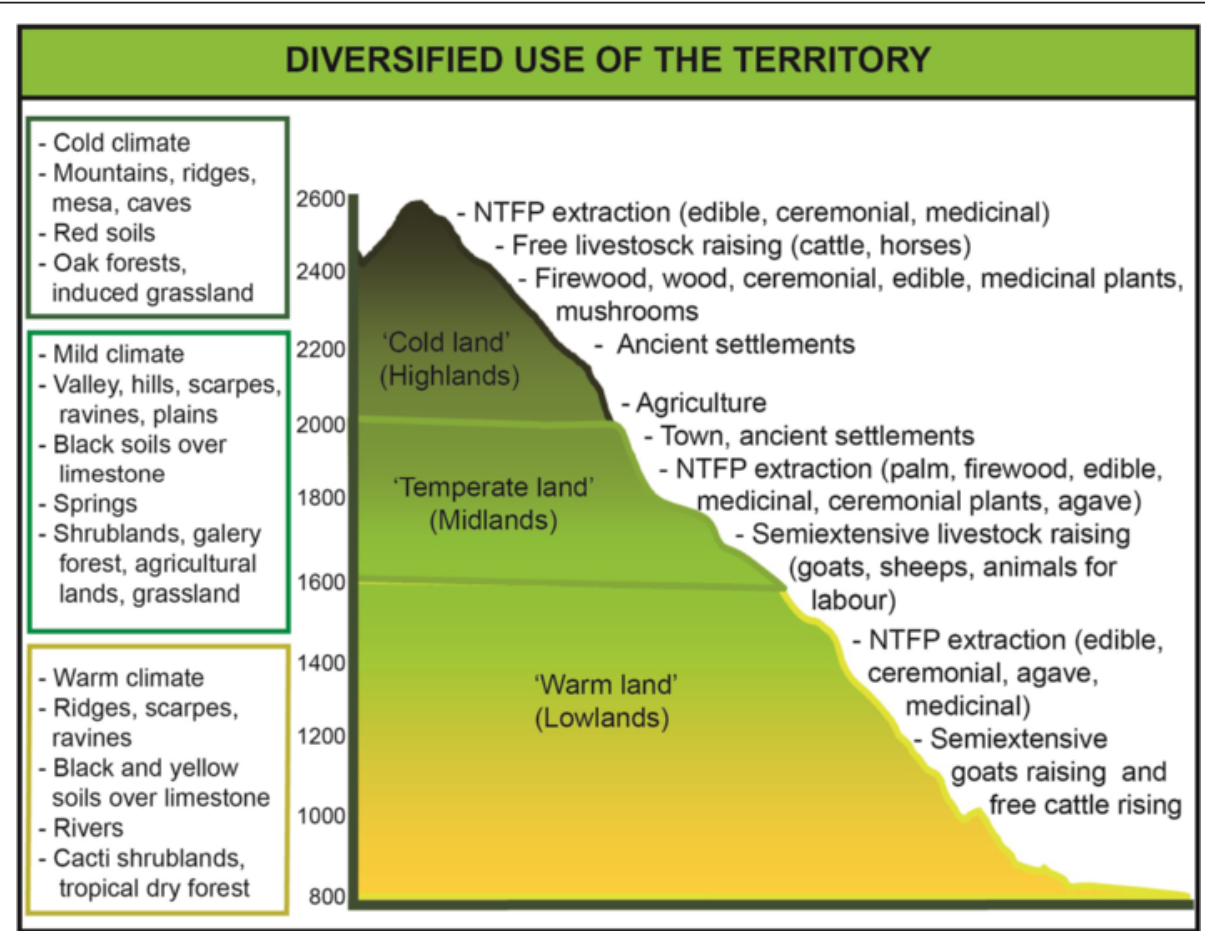

Fig. 3 Characteristics of landscapes, general environmental units recognized by people in the territory of Santa María Ixcatlán and plant resources use 

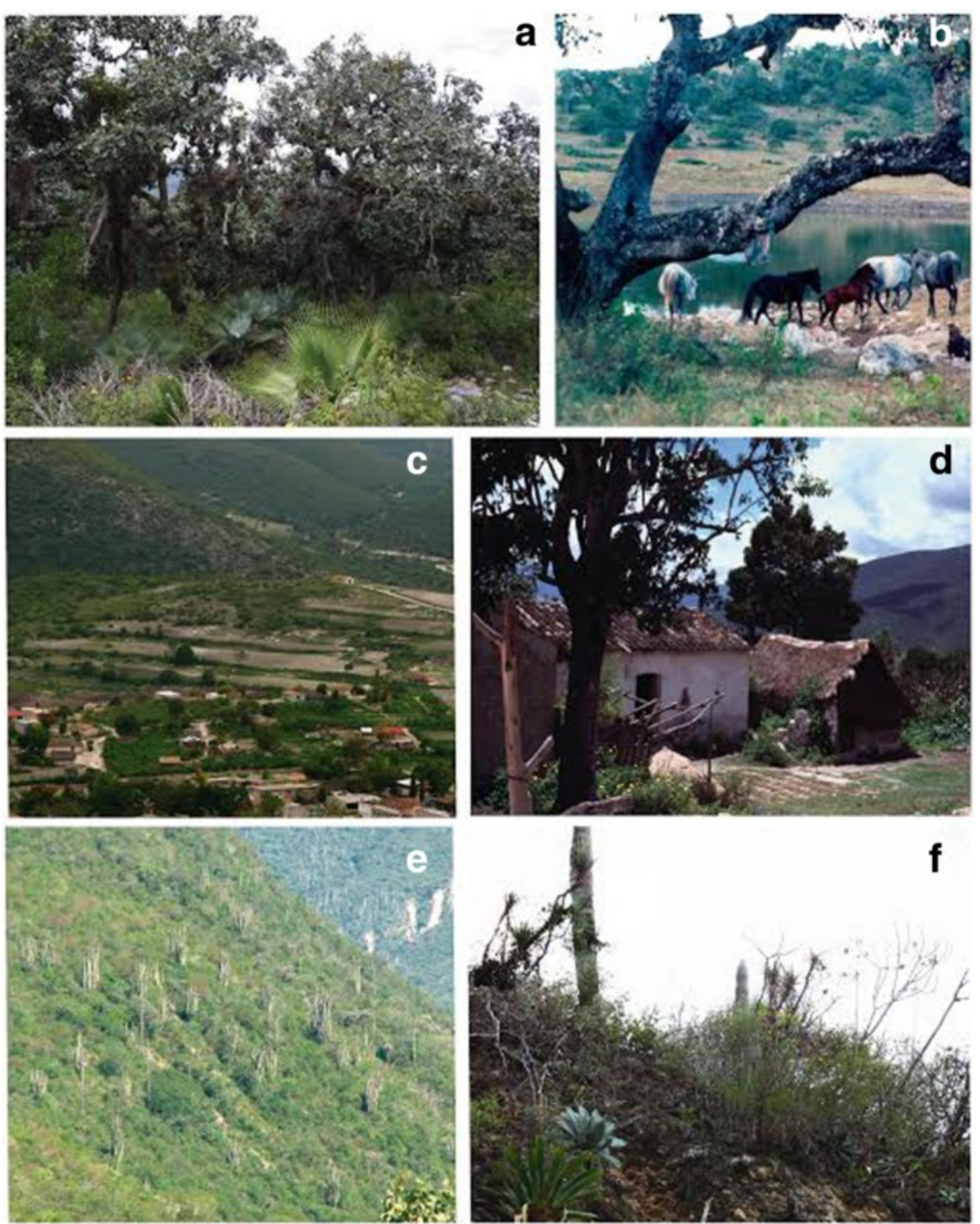

Fig. 4 View of environmental units. a) Quercus liebmanni and Quercus laeta forest; b) Dam "La Laguna", grassland and oak forest remnant; c) Homegardens, agricultural fields, palm scrublands and mexical in the southwest side of town; d) View of a homegarden and a traditional house with roof of palm leaves; e) Pseudomytrocereus fulviceps shrubland; f) Tillansia grandis and Agave potatorum in Cephalocereus colummna-trajanni shrubland

allowed for all community members; however, the relation between mescal producers and communal authorities has become tense in the last years, since federal environmental authorities are trying to regulate this activity in the region. Since 2011 some mescal producers started to enhance the availability of agaves near their houses or agricultural fields by spreading seeds or cultivating them in homegardens and green houses. Some mescal producers have participated in exchanges of experiences for agave management with other communities, and governmental programs have promoted some actions as reforestations and the construction of a communitarian greenhouse that stared to produce agave plants in 2015.
The activities described are supported by using different environments and sites of the territory (Figs. 3 and 4). The whole territory is of common use, but knowledge about distribution, abundance and quality of plant resources are recognized as basic issues to access to any locality and its resources. The subsistence strategy is complemented by economic subsidies from governmental programs for elderly, child scholarships, creole seeds conservation, and agriculture and stockbreeding development (Fig. 2). In 2000 assistance program started to support the $45 \%$ of households, by 2012 nearly $95 \%$ of the households received monetary incomes from those programs. In almost a half of the households at least one member has temporal or 
occasional employments at town that allow them to get additional monetary incomes (Fig. 2). Although irregularly, some migrants support their families to pay communal fees for celebrations, maintaining religious monuments and building public infrastructure (Fig. 2).

\section{Plants use}

We inventoried 780 vascular plants species belonging to 119 botanical families; 589 of them are native to Ixcatlán, and the other 191 have been introduced from other parts of Mexico and the world (Appendix). In order to satisfy their broad spectrum of needs people make use of 627 plants species with one to 27 use categories (Table 2), 267 species have one use and 360 have between 2 and 11 different use types.

Table 2 Use categories of Santa María Ixcatlán plant species. Data according to 62 people interviewed in 130 work sessions

\begin{tabular}{|c|c|c|c|}
\hline Use & Native & Introduced & Total \\
\hline Fodder & 238 & 30 & 268 \\
\hline Ornamental & 160 & 110 & $270^{a}$ \\
\hline Medicinal & 166 & 53 & 219 \\
\hline Edible & 72 & 66 & 138 \\
\hline Ceremonial & 73 & 55 & 128 \\
\hline Firewood & 44 & 2 & 46 \\
\hline Utensils & 29 & 4 & 33 \\
\hline Living fences & 24 & 6 & 30 \\
\hline Timber products and construction & 27 & 2 & 29 \\
\hline Shade & 12 & 11 & 23 \\
\hline Food aditive (flavor) & 9 & 6 & 15 \\
\hline Handcrafts & 11 & 1 & 11 \\
\hline Insects repellent & 8 & 0 & 8 \\
\hline Soil control & 6 & 2 & 8 \\
\hline Animals medicine & 1 & 1 & 6 \\
\hline Facilitator $^{b}$ & 3 & 2 & 5 \\
\hline Toys & 5 & 5 & 5 \\
\hline Alcoholic beverages & 2 & 1 & 3 \\
\hline Cosmetic & 2 & 1 & 3 \\
\hline Soap & 2 & 1 & 3 \\
\hline Paint & 3 & 0 & 3 \\
\hline Weather predictors & 2 & 0 & 2 \\
\hline Aromatizing & 1 & 0 & 1 \\
\hline Tannin source & 1 & 0 & 1 \\
\hline Water attracter & 1 & 0 & 1 \\
\hline Glues & 1 & 0 & 1 \\
\hline Poisons & 1 & 0 & 1 \\
\hline Unknown & 150 & 3 & 153 \\
\hline TOTAL & 589 & 191 & 780 \\
\hline
\end{tabular}

$\mathrm{a}=132$ species are considered "luxury of houses", 80 as "luxury of the mountain", and 59 as "luxury of houses and mountain"; $b=$ Plants used as stake, hosts and nurse plant

\section{Fodder}

A total of 268 plant species are consumed by domestic animals (Table 2, Appendix). 238 species being native to Ixcatlán and 165 of them have other uses mainly as edible, medicinal or as ornamental plants. Of the 30 introduced species 15 are propagated, and some of them are highly valued (Appendix). Zea mays is the most valuable specie as fodder, its stubble is used by the $80 \%$ of households and during periods of scarcity, $87 \%$ of the households have to buy it to regional sellers (Fig. 2, Appendix). Other important introduced plants are Avena fatua and Hordeum vulgare which are cultivated specifically for this use.

\section{Ornamental}

Ixcatlán people name as "luxury" ('lujo' in Spanish) the plant species that embellish or adornment houses, homegardens, agricultural fields and landscapes, in the two last cases these plants are called "mountain luxury". High variation was documented about which plants are considered as luxury, as most consultants said "it is something that depends on the appreciation of beauty of things by each person". People consider that luxury plants embellish the house, calls friendship, invites people to come into the house, allows to strength the heart or spirit and it is motive of proud for the owner. The importance of maintaining these plants varies among people, but generally are appreciated because in addition to the quality of embellish, these plants provide shade, good sites for resting and well-being or are used as fodder, edible and medicine. Nearly 270 species were recognized for its quality of embellish, 160 of them are native to Ixcatlán, 37 of them are not used in other form. 19 luxury plant species are transplanted from forest to houses or are propagated through sexual or asexual propagules. Introduced plants are highly valued (Table 2 , Appendix), and are common gift of outsiders that visit the town, or these are obtained through governmental programs or by interchanging palm leaves with outside sellers.

\section{Medicinal}

We documented 219 species used as medicine (Table 2), 61 of them exclusively used with this purpose, the rest have other uses mainly fodder, edible or are considered as "luxury plants". The medicinal plants commonly are used to treat stomach-ache, cold, fever, ear pain, sprains, and cultural illnesses like "sustos" (shocks caused by impressions), "aires" (malaise caused by uncomfortable situations) and "alferecia" (weakness, loss of appetite and irritability in children). Although knowledge about plants used in childbirth is extensive, few young women recognize to use them. In 2000, all people said to use medicinal plants, but in 2012, $15 \%$ of people interviewed said they only use allopathic therapies and the rest said to combine traditional and institutional medicine. Of the 53 introduced species some are highly valued for their 
medicinal use (Table 2, Appendix) and are cultivated to have them available as it is the cases of Matricaria chamomilla, Tanacetum parthenium and Artemisia ludoviciana.

\section{Edible}

We documented 138 plant species used as food, 99 of them have other uses, mainly as fodder, medicinal and ornamental (Appendix, Table 2). Nearly 50 species complement the diet of people which is based on maize tortillas, beans and chili sauces; 66 introduced edible species are cultivated, as it is the cases of maize, beans, vegetables, condiments and fruits (Appendix). These plants are available in the local stores but people say "the little that we harvest is a saving, these plants are things that we do not have to buy". Other reasons for cultivating are quality; people argued that vegetables locally produced are of better quality than others from outside particularly Coriandrum sativum and Solanum lycopersicum, they consider that local products have better taste, smell and texture.

\section{Ceremonial}

A total of 128 plant species are used to offer them to Catholic Saints in altars at homes, hermitages, thumbs, and the church. Some are used in ceremonies and processions (Table 2, Appendix); 117of them have other uses, 95 are used as ornamental or luxury (Table 2). The introduced plants are highly appreciated (Appendix), and particularly cultivated for their flowers, like Tagetes erecta used by $95 \%$ of households during the great feast of the Day of the Dead (Appendix). People recognize several varieties according to the size, colour and form of flowers, and it is common to store seeds of their favourite variants to be propagated in the next cycle. Local interchange of ceremonial plants flowers is common among households as gifts or trade, especially of introduced species as Tagetes erecta, Zantedeschia aethiopica, Leucanthemum maximum, between others.

\section{Firewood}

We recorded 48 species used as firewood (Table 2), 44 of them are native species, and 46 have other uses. These are the main source of cooking energy (only $35 \%$ of households have gas stoves, but all use firewood for cook "maize tortillas"), and is the unique fuel to mescal production and for baking bread. In the year 2000, consumption of firewood per household was of 143.4 \pm $11.3 \mathrm{~kg} /$ week, and in 2012 it was $108.8 \pm 12 \mathrm{~kg} /$ week, a decrease apparently due to a governmental program for installing efficient stoves. For mescal production the consumption increased from 16.2 ton in 2000 to 63.36 ton in 2012; nearly $52 \%$ of these quantities is from alive oaks, which is considered the appropriate wood for baking the agave stems in the process of mescal production.

\section{Plant management}

Nearly $82 \%$ of all plants species recorded (636 spp.) are recognized to be under interventions by humans or foraged by domestic animals (Appendix); 424 of them are managed through at least two different practice types and 401 species are under practices directed to maintain or increase their availability.

Gathering is the most common practice for obtaining products of native plants and it is the only practice for 83 species (Table 3). This practice was documented among wild and introduced species, some of which have become naturalized (Appendix). We recorded 251 native and introduced species having special protection (Table 3). In homegardens and agricultural fields protection comprises actions like irrigation, exclusion from herbivorous and competitors, nursing, adding of livestock manure, protection against frost, weeding, pruning, and providing or removing shade. In communal lands, protection of native plants is conducted by avoiding pastoral routes in sites where people know valuable plants occur. Also, the Communitarian Assembly construct regulations for protecting some species, based on principles of favoring direct consumption by local people, forbidding extraction for commercialization and cutting of alive trees. However these regulations as practices directed to prevent unnecessary damage not always are followed.

In total, 206 species are tolerated during clearing vegetation in homegardens and agricultural fields. The main reason is its utility, but 23 species that are not used are tolerated since people said that "plants could be useful in the future", and "do not interfere with the development of other plants" or because "plants have the right to live" and "are part of nature".

Propagation of 155 species is carried out by seeds, bulbs, corms, rhizomes, tubers, pseudo-bulbs, bulbils, plantlets, shoots, cladodes and sticks; 33 of them are native wild species used mainly as ornamental. Complete individuals of 139 species are transplanted, 71 of them

Table 3 Plants management practices realized substitute "realized" by "carried out" in Santa María Ixcatlán

\begin{tabular}{llll}
\hline Management practice & Native & Introduced & Total \\
\hline Gathering & 281 & 18 & 299 \\
Foraging & 223 & 20 & 243 \\
Tolerance & 152 & 54 & 206 \\
Protection & 91 & 160 & 251 \\
Trasplanting & 71 & 68 & 139 \\
Uproot & 63 & 13 & 76 \\
Propagation & 33 & 122 & 155 \\
Enhancement & 9 & 25 & 34 \\
Unknown & 143 & 1 & 144 \\
\hline
\end{tabular}

Data according to 62 people interviewed in 130 work sessions 
from wild populations in forests to homegardens and agricultural fields. Occasionally, some epiphytic bromeliads and orchid species are relocated from one branch or tree to other, when their host's branches are cutting to allow their survival.

The abundance of 26 species or some variants is promoted by tolerating them until seed production, and in some cases seeds are collected, stored and then sown or dispersed; 76 species (63 of them native) are constantly uprooted in agricultural fields and homegardens (Table 3), some of them are also under practices to maintain them and ensure their availability.

\section{Biocultural importance \\ Fodder}

Variation in biocultural importance of 238 fodder native species is mainly explained by management type and number of uses ( $38 \%$ of variation in the first principal component), and cognitive prominence and consumption ( $22 \%$ of variation in the second principal component; Table 4). Species with the highest biocultural importance (blue circle in Fig. 5a) are subject to several management practices, but its use as fodder is low with the exception of Quercus liebmani whose acorns are gathered and stored for feeding pigs, and inflorescences of Agave spp. that are occasionally consumed by cattle. Simsia lagascaeformis and Tithonia tubaeformis (pink circle in Fig. 5a) are the species with highest cognitive value, and are tolerated in homegardens or agricultural fields, where these are also uprooted to control their abundance. Similar situation occurs with Amaranthus hybridus, Mirabilis xalapa, Sicyos laciniatus and grass species (green circle in Fig. 5a).

Legumes, oak acorns, herb species and grasses are the main fodder for cattle, goats and sheep. Management practices to ensure their availability are poor or absent (orange and brown circles in Fig. 5a). Tillandsia gymnobotrya and Hechtia oaxacana are highly valued as fodder, substituting maize stubble (green circle in Fig. 5a). Shepherds drop the epiphytic plants for cattle and goats, and nearly $30 \%$ of households gather and carry them to town for feeding donkeys and horses, extracting 800 to 1920 individuals per year.

\section{Ornamental plants}

Biocultural importance of 160 native ornamental plants is explained mainly by their management complexity and number of uses ( $40 \%$ of the variation explained by the first principal component), and ecological importance and management ( $25 \%$ of variation explained by the second principal component) (Table 4). The most important plant species (Brahea dulcis, Juniperus flaccida, Quercus liebmanni, Morus celtidifolia and Agave potatorum), with exception of Morus celtidifolia are considered "luxury of the mountain", all of them are highly valued because of their multiple uses, and have high ecological importance (blue circle in Fig. 5b).

Oaks, grasses and numerous plant species producing beautiful flowers are appreciated to embellish the wilderness and some of them are maintained for this appraisal on agricultural fields or protected against livestock, as it is the case of the terrestrial orchids (Cyrtopodium macrobulbon and Govenia lagenophora), among others (brown circle in Fig. 5b).

Some valuable "luxury of the mountain" plants, are carried to homegardens; for instance, Euchile karwinskii, several spherical and barrel cacti species (Mammillaria spp., Coryphantha retusa, and Ferocactus spp.), Crassulaceae species, Tillandsia spp., among others. These plants are propagated and maintained for embellishing the house and 42 species are used for ceremonial purposes too (green circle in Fig. 5b).

\section{Medicinal plants}

The biocultural importance of the 166 native medicinal plant species is explained mainly by their complexity and site of management, and their cognitive prominence in the first principal component (43\% of variation). Number of uses, ecological importance, consumption and cognitive importance are important in the second principal component (29\% of variation) (Table 4). In general, native plants with the highest biocultural importance like

Table 4 Contribution of socio-ecological factors to explain the variation of native plant species biocultural importance

\begin{tabular}{|c|c|c|c|c|c|c|c|c|c|c|c|c|}
\hline \multirow{2}{*}{$\begin{array}{l}\text { Use type } \\
\text { Factor }\end{array}$} & \multicolumn{2}{|c|}{ Fodder } & \multicolumn{2}{|c|}{ Ornamental } & \multicolumn{2}{|c|}{ Medicinal } & \multicolumn{2}{|l|}{ Edible } & \multicolumn{2}{|c|}{ Ceremonial } & \multicolumn{2}{|c|}{ Firewood } \\
\hline & $\overline{P C 1}$ & PC2 & $\overline{P C 1}$ & PC2 & $\overline{P C 1}$ & PC2 & $\overline{P C 1}$ & PC2 & $\overline{P C 1}$ & $\mathrm{PC2}$ & $\overline{P C 1}$ & PC2 \\
\hline Cognitive importance & -0.09 & 0.78 & 0.55 & 0.31 & 0.72 & -0.58 & 0.44 & -0.18 & 0.54 & 0.24 & 0.69 & -0.17 \\
\hline Consump-tion & 0.04 & 0.77 & 0.55 & 0.12 & 0.63 & -0.64 & 0.39 & -0.32 & 0.35 & -0.63 & 0.33 & 0.67 \\
\hline Number of uses & 0.76 & 0.16 & 0.74 & 0.47 & 0.52 & 0.69 & 0.47 & 0.73 & 0.65 & 0.61 & 0.75 & 0.29 \\
\hline Ecological importance & 0.48 & 0.21 & 0.53 & 0.52 & 0.31 & 0.65 & 0.32 & 0.82 & 0.51 & 0.68 & 0.61 & 0.57 \\
\hline Management complexity & 0.93 & -0.01 & 0.81 & -0.52 & 0.82 & 0.33 & 0.93 & -0.13 & 0.89 & -0.29 & 0.9 & -0.24 \\
\hline Management site & 0.76 & -0.22 & 0.59 & -0.76 & 0.8 & -0.01 & 0.78 & -0.36 & 0.69 & -0.58 & 0.69 & -0.66 \\
\hline
\end{tabular}

Data are correlation values between variables and the first two components of Principal Components Analysis PCAs. Values in bold have high influence in principal components, therefore in the classification of biocultural importance too 


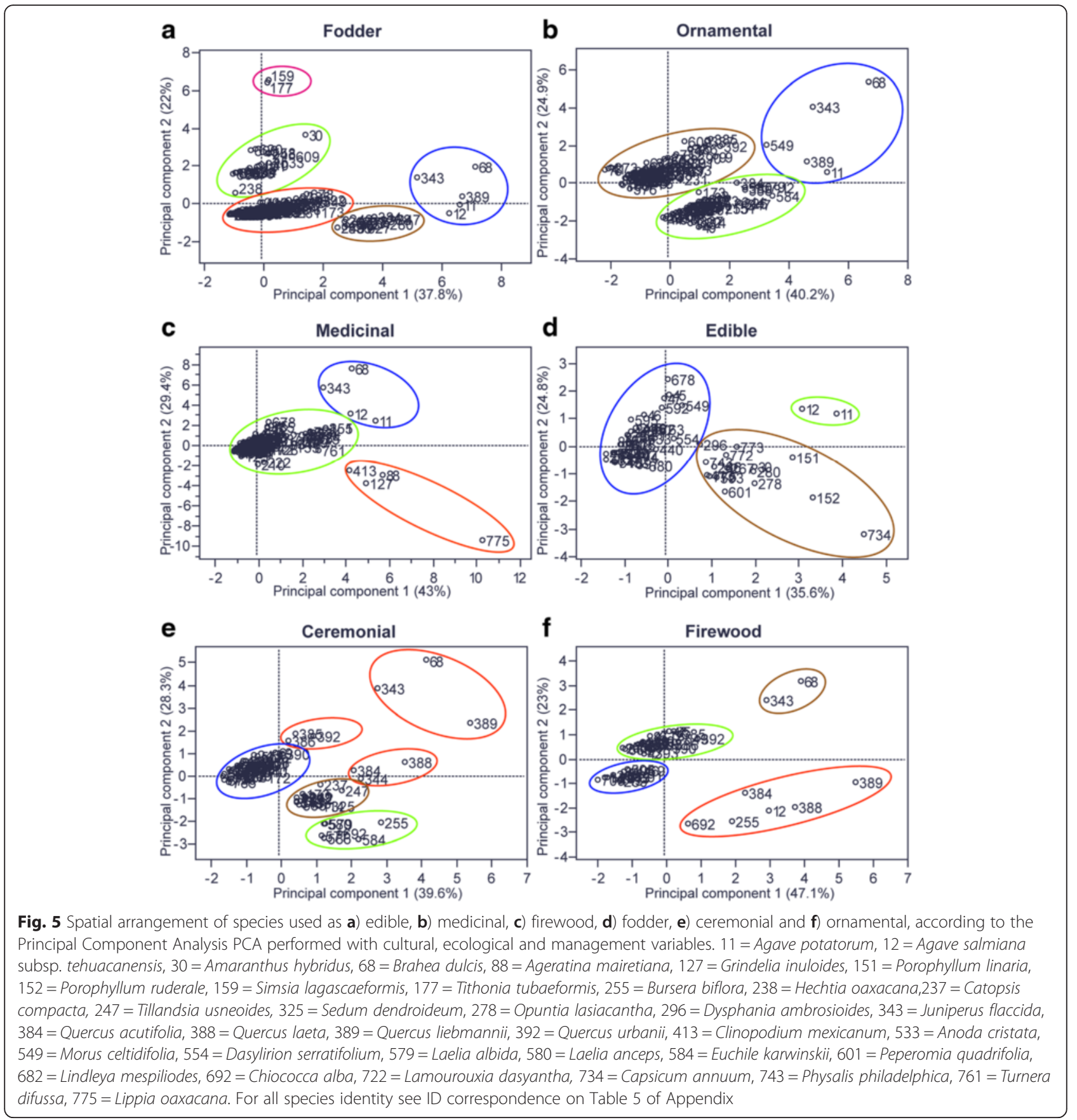

Lippia oaxacana, Ageratina mairetiana, Grindelia inuloides and Clinopodium mexicanum have few uses, high cognitive prominence and low ecological importance (orange circle in Fig. 5c). These plants are mainly gathered and stored to ensure their availability when it could be necessary. Some people have propagated these plants but said that "they are experimenting" but "quality of plants growing in nature is better than the cultivated ones".

There is another group of plants like Agave spp., Juniperus flaccida and Brahea dulcis, which have high ecological importance, are subject to complex management and used with numerous purposes, and occasionally used as medicine (blue circle in Fig. 5c). The rest of the species (green circle in Fig. 5c) are occasionally consumed, collected when they are needed, and some of them are also valued for other types of use.

\section{Edible}

Principal components analysis shows that biocultural importance of the 72 native plants is explained mainly 
by management practices complexity and management site (ex situ or in situ) in the first principal component (36 \% of variation), and ecological importance and number of uses in the second principal component ( $25 \%$ of variation) (Table 4). Native plants with higher biocultural importance are those with greater management complexity, consumed by more families and have few uses, regardless of their ecological importance (brown circle in Fig. 5d).

One of the most important plant species is Capsicum annuum, consumed by all households, mainly getting it by interchange, but it is also cultivated in homegardens but the wild variety is rarely gathered. Species like Porophyllum ruderale, Porophyllum linaria, Amaranthus hybridus, Opuntia lasiacantha, and Dysphania ambrosiodes are consumed by nearly all households and their contribution to diet is greatly important. For instance, the green Amaranthus hybridus is consumed on average $14.4 \pm 2.4$ times per year from June to September, almost always together with Porophyllum linaria; Dysphania ambrosiodes is cooked with beans and consumed every day by all households. These species are subject to management in agricultural fields and cultivated in homegardens to ensure their availability and to have them close and in case of scarcity are getting in the stores. Physalis philadelphica is consumed in sauces almost always raw to allow its seeds to germinate after dispersed when washing dishes in homegardens, where plants of this species are tolerated, transplanted and protected.

Other species are obtained by gathering (blue circle in Fig. 5d). Some of the most valuable (e. g. Dasylirion serratifolium and Peperomia cuadrifolia) are consumed by nearly all households and commonly are shared with relatives, especially elders who are unable to get them by themselves. Some people have tried to propagate them in homegardens but they said that their experiments were unsuccessful because they obtain low production, it was difficult to maintain them, and require long time to harvest their products. Agave species are grouped (green circle in Fig. 5d), have high biocultural values, are intensely managed, abundant and highly valued for multiple purposes, although the consumption of its flowers as food is currently uncommon.

\section{Ceremonial plants}

Variation in biocultural importance of the 73 native species is mainly explained by management complexity and number of uses in the first principal component $(40 \%$ of variation), ecological importance, consumption and number of uses ( $28 \%$ of variation explained by the second principal component; Table 4). The species with the highest biocultural value were those more intensely managed and valued for other uses (orange circles in Fig. 5e), for instance oaks that are part of the game of "El palo" played in the celebration of the Day of the
Dead, when teams of young men go to the forest to cut whole dead trees and carry them on to the town to be fired in front of the church. Other examples are Brahea dulcis leaves, which are used to weave shoes for deceased people and Juniperus flaccida whose resin is used when Bursera resin is scarce or unavailable.

The most cognitively salient species are appreciated for their flowers smell and beauty (green circle in Fig. 5e), which receive management practices and are extensively used regardless of their low ecological importance. In the extraction of orchid flowers people take care of leaving some bulbs, and after their ceremonial use, their bulbils are transplanted in homegradens as it occurs in the case of Euchile karwinskii. Laelia albida is cultivated in $65 \%$ of homegardens and Laelia anceps in $35 \%$ of them, this management is motivated by the appreciation of their beauty and scarcity in forests. Resin of Bursera biflora is particularly appreciated and used in a high number of rituals, this tree species is protected in situ, cannot be tamed or even damaged for extracting its resin and most people use only the resin of those trees naturally injured by insects located in warm lands to assure the resin quality (Fig. 3). Other species like Chiococca alba, Rhynchostele maculata and Epidendrum radioferens are highly valued and frequently used species but rarely transplanted into homegardens, in part because people consider they are abundant, but in part because of the difficulties for their propagation. Some species are used to embellish the "Nativity scenes" (Mammilaria spp., Catopsis compacta, Tillandsia spp.) are transplanted in homegardens after their use (brown circle in Fig. 5e). Most of ceremonial species are only gathered as it is the case of Lamourouxia dasyantha (blue circle in Fig. 5e) and in many cases are shared with relatives, especially old people.

\section{Firewood}

Principal components analysis shows that biocultural importance of plants used as firewood is mainly explained by the complexity of their management in the first principal component (47\% of variation), and consumption and ecological importance in the second component (23\% of variation) (Table 4). Species used as firewood with the highest biocultural importance are oaks Quercus spp. (orange circle in Fig. 5f), which are consumed by all households, and have the highest cognitive prominence. Oaks are tolerated and protected in agricultural fields, and sometimes people transplanted and take care of them in their houses as ornamental plants. In this group, Agave salmiana subsp. tehuacanensis is valued as good firewood, but its use is uncommon since people prefer to use its dry stalk for house construction. Two important species used as firewood are Brahea dulcis and Juniperus flaccida, which are intensely managed in agricultural fields and homegardens, have high ecological importance, are 
frequently used, and are highly culturally valued because of their multiple uses (brown circle in Fig. 5f).

The remaining species receive poor management (green and blue circles in Fig. 5f) and differ in their consumption, cognitive prominence and ecological importance. Some of these species have high biocultural value (Quercus urbanii, Quercus castanea, Quercus conspersa, Rhus chondroloma, Rhus standleyi, and Morus celtidifolia; green circle in Fig. 5f).

Although of the most valuable species for all interviewees are Quercus spp., Arbutus xalapensis and Juniperus flaccida, the "charges" (measurement unit which is the amount of material that a donkey is able to carry) composition highly varied among households, oaks being on average $(\bar{X}=79 \%)$, the rest are at least 30 species of shrubs managed in agricultural fields and homegardens being Dodonaea viscosa, Acacia spp., Comarostaphylis polyfolia, Eysenhardtia polystachya, and Garrya ovata, among the most common species.

\section{Discussion and conclusions}

\section{Subsistence strategy}

The multiple use of resources that including a great variety of ecosystems and resources and characterizing the Ixcatec subsistence are expressions of common patterns of interactions between humans and plants found among indigenous peoples of Mesoamerica [1, 3, 39, 46-49]. Such pattern is particularly important in a region like the Tehuacan Valley where the scarcity and uncertainty of rainfall and agricultural yield are also characteristic [17, 33, 39, 50]. Interchange of natural resources in the regional markets for obtaining staple food and other goods is clearly a strategy to face problems of availability of resources since preColumbian times [51]. For instance, commercialization and barter of local products like palm leaves, hats, mescal, and domestic animals, is a common strategy in numerous Mesoamerican communities [52-54] and many rural regions in the world to deal with the uncertainty [55].

Other activities like commerce and income subsidized by governmental programs, are part of the process of adaptation that may contribute to face eventual environmental and social adversities, similarly as recently documented among Mayan communities in southern Mexico [53]. The assistance support programmes from Government are progressively more important in the local subsistence strategies, but also, these programmes represent risks for the systems of management of natural resources, as it has been documented for programmes supporting agriculture, which promote the removal of trees and shrubs in agricultural land, thus affecting the maintenance of agroforestry systems $[8,21]$. Seasonal employments allow solving some problems [17], but also these may cause the regardless or abandonment of traditional activities, the loss of TEK and, in some cases, the abandonment of the community.

\section{Management diversity}

The widely management practices set and other cultural and social strategies documented have allowed to maintain plant species that sustain the multiuse subsistence strategies as it has been reported at regional level $[11,56,57]$

At regional level, gathering and foraging of plant resources by humans and their domestic animals are the most common and simple form of interaction between social and ecological systems [56], but for most useful species recorded people carry out practices directed to maintain and ensure their future availability [11], and a broad variety of strategies are being carried out for such a purpose [17]. These general trends were observed in Santa María Ixcatlán, is practiced in an even higher percentage of plant species (nearly $65 \%$ ), which is an expression of the particularly deep of TEK developed by the Ixcatec.

Management practices such as tolerance, enhancing, protection and cultivation (by sowing, planting or transplanting) look for ensuring availability of plant resources and controlling its uncertainty, are primary mechanism in the domestication process for some species $[10,58]$. It has allowed through selection of particular individual (phenotypes) and germplasm to start cultivation, maintaining and continuing processes of domestication. These processes were evident in the staple crops, as well as in wild and semi-domesticated Physalis philadelphica, Tagetes erecta and Cosmos bipinnatus in which selection to satisfy particular flavours, colours, and size, among others characteristics is carried out by people.

The socio-cultural strategies documented in all types of use as it is the mobility in resource gathering of valuable species, the diversification of resources to satisfy a need, and the substitution of one species with another or with other materials, have been recognized as buffer mechanisms to uncertainty $[17,59]$. Other important strategies based on social interactions as was the interchange of plants as gifts and interchange of information about management techniques, allow important diffusion of experiences among households and communities and are important mechanisms of social cohesion, an important issue to maintain traditional institutions $[17,60]$. Strategies associated to governance as it is the case of regulations are being effective for conserving some species. This is for instance the case of Litsea glaucescens and several oak species Quercus spp., whose populations are conserved in Ixcatlán through local regulations that only allow the extraction for direct consumption by househlds, but in other villages of the region have been severely affected and became extinct $[15,16]$. However, in other species regulations have been ineffective for controlling new intensities of extraction required because of socio-economic needs. This is clearly the case of Agave potatorum in which the increasing demand of mescal has been for the moment higher than the capacity for collective regulations and technical responses. 
Other interactions like removal (uprooting), opposed to maintenance, shows the complexity of interactions between humans and plants and the importance of detailed knowledge that people may have to take into account to make a decision based in the balance of the negative effects and utility that these species could provide [15]. For instance, in some cases like Thitonia tubaeiformis, Amaranthus hybridus and other weeds, which are valuable plants, people control its abundance inside of the agricultural field at begging the cycle in order to prevent competition with maize, but at the same time protect them in the borders to prevent fodder scarcity just in case that maize straw become scarce or to ensure the availability of greens.

The management practices have involved the transformation of ecosystems through intentional or incidental changes in the composition and structure of vegetation, the modification of relief, hydrological systems and biogeochemical processes in soils [61]. Concrete examples of this process are the creation and maintaining of secondary vegetation as induced grasslands and palm scrubland, changes in vegetation structure in forest zones where grazing routes are, erosive process in current and abandoned agricultural fields, and engineering works to retain soil and water for agriculture and livestock (Figs. 1, 2 and 3). Homegardens, crop fields and pasturelands distributed in the three types of environments recognized by the Ixcatec within their territory (Fig. 3), have originated a great variety of landscape units where management of wild and domesticated plant species take place, conforming forest, agroforestry, agro-silvo pastoral, and silvo-pastoral systems $[62,63]$. In these systems people maintain a high level of biodiversity; for instance, on average people of Ixcatlán maintain 29 woody native species in their agricultural plots [22]. These systems are biocultural expressions and areas continually generating new biocultural diversity through also continual observation and experimenting management techniques $[8,64]$. In the palm scrublands, for instance, which are highly important for the Ixcatec, people have shaped their conformation managing Brahea dulcis in order to increase its availability in agricultural and fallow plots, as well as in homegardens. This practice has happened most probably since pre-Columbian times, since this species is important for Ixcatec people [51, 52].

The role of plant species in the Ixcatec subsistence and in the interactions of humans to conserve plant resources may define particularities of their own culture $[3,65]$. Management of some plant species is closely related with the form of preparation of food stoves, as it was described for Physalis philadelphica. Relation of the Ixcatec with the palm Brahea dulcis is particularly significant, this species is part of almost all activities in their daily life, and it has been considered as an indissoluble element of Ixcatec culture [32, 33, 51, 52, 66, 67].
The high levels of diversity and interactions documented in Ixcatlán compared with the regional flora (30\% of the total regional flora, $36 \%$ of all useful plants recorded in the region, and $66 \%$ of managed species identified in the Tehuacán Valley) [11, 24, 25], confirm the importance of the Ixcatec biocultural heritage and the character of the Tehuacan Valley as a priority biocultural region of Mexico [3].

Our research and sampling effort is one of the highest carried out by ethnoecological studies in the Tehuacán Valley [11, 15-17, 56, 68-71]. This fact confirms that it is still needed continuing efforts to documenting TEK, biocultural processes of diversification and their connection with management innovation and domestication. In this region, archaeological records in caves has been source of information about biocultural construction since prehistory, whereas local studies should continue documenting one of the areas with highest richness of ethnobotanical knowledge of Mexico and a place where ongoing processes for sustainable resource management and local processes of domestication are taking place.

\section{Biocultural importance}

The integration of socio-cultural and ecological variables for understanding the importance of plant species, follows the proposal by Castaneda and Stepp [72] for estimating ethnoecological importance. Our evaluation found that variables associated to management complexity are in general those more contributing to explain the variation in the first principal component of the six use categories analysed. This fact suggests that management is representative of the socio-ecological factors interacting and mutually influencing their properties [73]. In other words, studying management of natural resources is a good methodological basis for understanding socio-ecological systems and construction of biocultural heritage.

Brahea dulcis, Juniperus flaccida, and Agave salmiana subsp. tehuacanensis have particularly high biocultural importance values in almost all use types analysed. This fact is because of their multipurpose use, their cultural and ecological importance and their intensive management. The positive relation between cultural and ecological importance might be explained through the hypothesis of ecological appearance $[18,74,75]$, but we rather propose that the ecological importance currently observed is in part a result of ancient ecosystem management directed to increase their availability. The high resistance to disturbance, reproductive capacity of these species, among other ecological factors have favoured the enhancing of their abundance.

The relation between ecological and cultural importance varied in the different use types analysed. Among plants used as ceremonial and medicinal, the species with higher cognitive prominence and consumption have low availability, and their management is mainly through 
socio-cultural strategies, directed to ensure their availability, as the harvest technics to ensure their survival after the harvest, but not necessarily are directed to increase their abundance.

The number of uses was an important factor in edible, medicinal, fodder, ceremonial and ornamental plants; however, among medicinal plants, the species with higher cognitive prominence were those with few uses, in other words their properties determining them specialized medicinal plants, which is apparently related with their quality as resource [76].

Highly cognitive valued species not always are the most consumed or managed. For instance, species highly valued as ceremonial, like orchids have a low consumption because the difficulty to obtain them or be manipulated to increase their availability. These results and those found by several authors studying factors influencing management of edible plants $[15,77,78]$, indicate that management motives may be variable not only related with cultural importance and scarcity, which suggests the importance of continuing research in this line.

\section{Conclusion}

\section{Management factors and motives}

A case that allows observing how people dynamically construct processes of decision making about management is Agave potatorum, in which the perception of risk of disappearing of the resource is the main factor detonating management actions, as documented for other plant resources of the Tehuacán Valley [15]. The strategies developed depend on TEK of both species and ecosystems [17], but there are external factors influencing experimenting innovation in management actions, as illustrated in the cases of several species of Agave $[40,79]$, in which markets have influenced increasing of extraction and pressures on agave populations and new management techniques $[16,17,40]$. This case illustrates that crises detonate innovation, activating processes of experimenting, monitoring, adapting, testing and interchanging local and external experiences, as well as enhancing processes of social organization, collaboration with governmental and academic sectors, learning and adaptation, in which the communitarian platforms of dialogue are crucial for facing risks and uncertainty $[80,81]$.

In other cases, the uncertainty in the availability of highly valued resources are motives for managing other species with redundant use and are able to substitute particular desirable resources, as are the cases of Tithonia tubaeformis and Simsia lagascaeformis whose abundance is promoted in controlled ways before the uncertainty of the main fodder of the study area (maize stubble). Such a complex decision making has important consequences in households' economy [82] and biodiversity conservation in agroforestry systems $[21,22,83]$.
Uncertainty operates associated to several factors, and ensuring the products quality is another management motive. People prefer consuming their own crops, which are considered of better quality over those commercialized in stores. Practices to assure the quality not are exclusively on crop plants, others like Bursera biflora have specialized resin extraction techniques that take advantage of natural processes assuring the resin quality avoiding injure the trees, instead of cutting trunks, a common practice in other localities [84]. Moreover, the perception of quality loss discourages ex situ management, in addition to energy investment and difficulties involved in maintaining these species outside their environments, as was noted in Bursera biflora and medicinal plants.

The aesthetical sense, expressed by people that consider that plants embellish the spaces where they occur, as Cook noted [33] in mid 20th century, appears to be an important motive that determining the permanence of numerous native species in homegardens and crop fields as forests conservation. This motive has been reported by other authors in agroforestry systems of the region [21, 22], and our study suggests its high importance because of the high number of species considered as house or "mountain luxury", which receive some type of management practices.

Ethical principles like the fact that people recognize that plants are living beings with a right to exist, that plants should not be damaged because of whim, are ethical principles that motive management practices as tolerance. Also the including of several species in belief systems and matching cycles of plant management with the rituals calendar, suggest that although the Ixcatec kosmos is permeated by Catholic thinking, it maintains features with other Mesoamerican views of the world reported by other authors [20, 85].

Curiosity was mentioned to be involved in all management practices in response to motives such as uncertainty in plant resources' availability or aesthetical needs. It enhances testing new techniques or new species or be persistent when reproductive requirements make difficult the plants propagation.

Deepen the study of motivations and socio-economic and cultural factors that influence plant management allow understanding the processes of decision making construction and biocultural legacy. Such studies could provide unique opportunities for strengthening conservation strategies of sustainable forms of management of resources and ecosystems.

\section{Appendix}

Plant species of Santa María Ixcatlán. Species, number of uses, management, socio-cultural and ecological aspects; rarefaction curves of S Index, Ixcatec participants details, and botanical experts. 
Table 5 Species, Spanish common names, number of uses, percentage of families that consume it; cognitive prominence values expressed as $S=$ Sutrop relative prominence inde ${ }^{2}$ and biocultural importance expressed as first component value of the principal component analysis by use type (edible, medicinal, firewood, fodder, ceremonial and ornamental; distribution on vegetal types, importance ecological index value (EIVI); specie origin region, ecological status, management practices and management site with respect to species wild populations

\begin{tabular}{|c|c|c|c|c|c|c|c|c|c|c|c|c|}
\hline \multirow[t]{2}{*}{ ID } & \multirow[t]{2}{*}{ Family } & \multirow[t]{2}{*}{ Specie } & \multirow[t]{2}{*}{ Voucher number $^{a}$} & \multirow[t]{2}{*}{ Common name } & \multirow{2}{*}{$\begin{array}{l}\text { Number } \\
\text { of uses }\end{array}$} & \multirow{2}{*}{$\begin{array}{l}\text { Consumption } \\
\text { by use } \\
\text { (Households \%) }\end{array}$} & \multicolumn{2}{|l|}{ Fodder } & \multicolumn{2}{|c|}{ Ornamental } & \multicolumn{2}{|c|}{ Medicinal } \\
\hline & & & & & & & $\begin{array}{l}\text { Sutrop } \\
\text { Index } \\
\text { value }^{b}\end{array}$ & $\begin{array}{l}\mathrm{PC} \\
\text { value }\end{array}$ & $\begin{array}{l}\text { Sutrop } \\
\text { Index } \\
\text { value }^{c}\end{array}$ & $\begin{array}{l}P C \\
\text { value }\end{array}$ & $\begin{array}{l}\text { Sutrop } \\
\text { Index } \\
\text { value }^{d}\end{array}$ & $\begin{array}{l}\mathrm{PC1} \\
\text { value }\end{array}$ \\
\hline 1 & Acanthaceae & $\begin{array}{l}\text { Carlowrightia neesiana } \\
\text { (Schauer ex Nees) T.F.Daniel }\end{array}$ & SRL-1385 & & & & 0 & & 0 & & 0 & \\
\hline 2 & Acanthaceae & $\begin{array}{l}\text { Justicia candicans (Nees) } \\
\text { L.D.Benson }\end{array}$ & SRL-1395 & & & & 0 & & 0 & & 0 & \\
\hline 3 & Acanthaceae & $\begin{array}{l}\text { Justicia gonzalezii (Greenm.) } \\
\text { Henr. \& Hiriart }\end{array}$ & SRL-1333, SRL-1362 & & & & 0 & & 0 & & 0 & \\
\hline 4 & Acanthaceae & Justicia spicigera Schltdl & $\begin{array}{l}\text { SRL-92, SRL-188, ERL-41, } \\
\text { ERL-58, ERL-216, ERL-224 }\end{array}$ & Tintonil & 1 & & 0 & & 0 & & 0.0101 & \\
\hline 5 & Acanthaceae & Ruellia lactea Cav. & Photo record & & & & 0 & & 0 & & 0 & \\
\hline 20 & Aizoaceae & $\begin{array}{l}\text { Aptenia cordifolia (L.f.) } \\
\text { Schwantes }\end{array}$ & $E R L-46$ & & 1 & Ornamental $=6$ & 0 & & 0 & & 0 & \\
\hline 21 & Aizoaceae & Carpobrotus sp. & Photo record & & 1 & Ornamental = 6 & 0 & & 0 & & 0 & \\
\hline 22 & Aizoaceae & Mesembryanthemum sp. & ERL-213 & & 1 & Ornamental $=6$ & 0 & & 0 & & 0 & \\
\hline 28 & Alstromeriaceae & $\begin{array}{l}\text { Bomarea hirtella (Kunth) } \\
\text { Herb. }\end{array}$ & RLF-290 & & & & 0 & & 0 & & 0 & \\
\hline 29 & Amaranthaceae & $\begin{array}{l}\text { Alternanthera caracasana } \\
\text { Kunth }\end{array}$ & ERL-21, SRL-93 & Maravilla & 2 & & 0 & -0.216 & 0 & & 0 & -0.5049 \\
\hline 30 & Amaranthaceae & Amaranthus hybridus L. & $\begin{array}{l}\text { SRL-79, SRL-80, SRL-1122, } \\
\text { SRL-1141, ERL-74, ERL-102 }\end{array}$ & Quelite tintonil & 3 & $\begin{array}{l}\text { Fodder }=60 \\
\text { edible }=95\end{array}$ & 0.0207 & 1.4999 & 0 & & 0 & 0.6125 \\
\hline 31 & Amaranthaceae & Beta vulgaris $\mathrm{L}$. & Photo record & Betabel, acelga & 1 & & 0 & & 0 & & 0 & \\
\hline 33 & Amaranthaceae & Celosia argentea $\mathrm{L}$. & Photo record & Moco de pavo & 2 & $\begin{array}{l}\text { Ornamental }=18, \\
\text { ceremonial }=30\end{array}$ & 0 & & 0 & & 0 & \\
\hline 34 & Amaranthaceae & Gomphrena serrata L. & $\begin{array}{l}\text { RLF-60, RLF-242, SRL-90, } \\
\text { SRL-378, SRL-1175 }\end{array}$ & Gallitos & 2 & & 0 & -0.3826 & 0 & & 0 & -0.6606 \\
\hline 35 & Amaranthaceae & Iresine schaffneri S.Watson & RLF-320 & & 1 & & 0 & -1.0555 & 0 & & 0 & \\
\hline 36 & Amaranthaceae & Iresine sp. & SRL-1488 & & & & 0 & & 0 & & 0 & \\
\hline 26 & Amaryllidaceae & $\begin{array}{l}\text { Agapanthus africanus (L.) } \\
\text { Hoffmanns. }\end{array}$ & Photo record & Pando morado & 2 & Ornamental = 18 & 0 & & 0.0016 & & 0 & \\
\hline 23 & Amaryllidaceae & Allium cepa $\mathrm{L}$. & ERL-177 & Cebolla & 1 & Edible $=100$ & 0 & & 0 & & 0 & \\
\hline 24 & Amaryllidaceae & Allium sativum $\mathrm{L}$. & Photo record & Ajo & 2 & Edible $=100$ & 0 & & 0 & & 0.0016 & \\
\hline 37 & Amaryllidaceae & Crinum $\times$ powellii Hort. & ERL-237 & Azucena blanca & 2 & $\begin{array}{l}\text { Ornamental }=35, \\
\text { ceremonial }=11\end{array}$ & 0 & & 0 & & 0 & \\
\hline
\end{tabular}


Table 5 Species, Spanish common names, number of uses, percentage of families that consume it; cognitive prominence values expressed as $\mathrm{S}=$ Sutrop relative prominence index ${ }^{2}$ and biocultural importance expressed as first component value of the principal component analysis by use type (edible, medicinal, firewood, fodder, ceremonial and ornamental; distribution on vegetal types, importance ecological index value (EIVI); specie origin region, ecological status, management practices and management site with respect to species wild populations (Continued)

\begin{tabular}{|c|c|c|c|c|c|c|c|c|c|c|c|c|}
\hline 38 & Amaryllidaceae & $\begin{array}{l}\text { Hippeastrum puniceum } \\
\text { (Lam.) Voss Herb. }\end{array}$ & Photo record & Azucena roja & 2 & $\begin{array}{l}\text { Ornamental }=29, \\
\text { ceremonial }=11\end{array}$ & 0 & & 0.0168 & & 0 & \\
\hline 39 & Amaryllidaceae & $\begin{array}{l}\text { Hymenocallis harrisiana } \\
\text { Herb. }\end{array}$ & Photo record & & 1 & & 0 & & 0.0042 & -1.4444 & 0 & \\
\hline 40 & Amaryllidaceae & $\begin{array}{l}\text { Sprekelia formosissima (L.) } \\
\text { Herb. }\end{array}$ & Photo record & Azucena roja & 1 & Ornamental $=6$ & 0 & & 0.0078 & 1.0545 & 0 & \\
\hline 41 & Amaryllidaceae & $\begin{array}{l}\text { Zephyranthes minuta (Kunth) } \\
\text { D.Dietr.Herb. }\end{array}$ & Photo record & & 1 & & 0 & & 0.0037 & -2.0057 & 0 & \\
\hline 42 & Anacardiaceae & $\begin{array}{l}\text { Actinocheita potentillifolia } \\
\text { (Turcz.) Bullock }\end{array}$ & $\begin{array}{l}\text { RLF-109, RLF-274, SRL-1183, } \\
\text { SRL-1368 }\end{array}$ & Tetlate & 2 & Firewood $=100$ & 0 & & 0 & & 0 & -0.1037 \\
\hline 43 & Anacardiaceae & Cyrtocarpa procera Kunth & SRL-1358 & Chupandio & 1 & Edible $=20$ & 0 & & 0 & & 0 & \\
\hline 44 & Anacardiaceae & Pistacia mexicana Kunth & $\begin{array}{l}\text { RLF-326, SRL-1211, SRL-1340, } \\
\text { SRL-1523 }\end{array}$ & Socoya & 7 & & 0.0161 & 1.24 & 0 & & 0.0069 & 0.4921 \\
\hline 45 & Anacardiaceae & Rhus chondroloma Standl. & RLF-282, SRL-1222, SRL-1460 & Zumaque & 7 & Firewood $=100$ & 0 & 1.6290 & 0.0147 & 0.9307 & 0.002 & 0.6407 \\
\hline 46 & Anacardiaceae & Rhus standleyi F.A.Barkley & $\begin{array}{l}\text { RLF-59, RLF-255, SRL-269, SRL- } \\
472 \text {, SRL-1248, SRL-1470 }\end{array}$ & $\begin{array}{l}\text { Encino chaparro, } \\
\text { zomaque grueso }\end{array}$ & 3 & Firewood $=100$ & 0 & & 0 & & 0 & -0.043 \\
\hline 47 & Anacardiaceae & Rhus virens Lindl. ex A.Gray & $\begin{array}{l}\text { RLF-58, RLF-219, SRL-275, SRL- } \\
468, \text { SRL-1218 }\end{array}$ & Zumaque & 6 & Firewood $=100$ & 0 & 1.4214 & 0.0147 & 0.7703 & 0.002 & 0.5159 \\
\hline 48 & Anacardiaceae & Schinus molle L. & SRL-19, ERL-164 & Pirul & 4 & Ornamental $=6$ & 0 & & 0.0098 & & 0.0083 & \\
\hline 49 & Annonaceae & Annona cherimola Mill. & Photo record & Chirimoya & 1 & & 0 & & 0 & & 0 & \\
\hline 52 & Apiaceae & Ammi majus $\mathrm{L}$. & $\begin{array}{l}\text { SRL-24, ERL-13, ERL-81, Erl-131, } \\
\text { ERL-184 }\end{array}$ & Encaje & 2 & $\begin{array}{l}\text { Ornamental }=47 \\
\text { ceremonial }=20\end{array}$ & 0 & & 0 & & 0 & \\
\hline 53 & Apiaceae & $\begin{array}{l}\text { Apium leptophyllum (Pers.) } \\
\text { F.Muell. ex Benth. }\end{array}$ & SRL-1525 & & & & 0 & & 0 & & 0 & \\
\hline 54 & Apiaceae & Coriandrum sativum $\mathrm{L}$. & ERL-40, ERL-135, ERL-236 & Cilandro & 2 & Edible $=100$ & 0 & & 0 & & 0 & \\
\hline 55 & Apiaceae & Daucus carota $\mathrm{L}$. & Photo record & Zanahoria & 1 & Edible $=100$ & 0 & & 0 & & 0 & \\
\hline 56 & Apiaceae & $\begin{array}{l}\text { Eryngium bonplandii } \\
\text { F.Delaroche }\end{array}$ & $\begin{array}{l}\text { RLF-6, SRL-132, SRL-384, SRL- } \\
1247\end{array}$ & Ojo de gallo & 1 & & 0 & & 0 & & 0 & -0.9929 \\
\hline 57 & Apiaceae & $\begin{array}{l}\text { Eryngium comosum } \\
\text { F.Delaroche }\end{array}$ & RLF-127 & espinuda & 1 & & 0 & & 0 & & 0 & -1.0487 \\
\hline 58 & Apiaceae & $\begin{array}{l}\text { Eryngium pectinatum C.Presl } \\
\text { ex DC. }\end{array}$ & RLF-52, SRL-315 & & 1 & & 0 & & 0 & & 0 & -1.0295 \\
\hline 59 & Apiaceae & Foeniculum vulgare Mill. & SRL-72, ERL-229 & Hinojo & 2 & & 0 & & 0 & & 0.0119 & \\
\hline 60 & Apiaceae & $\begin{array}{l}\text { Petroselinum crispum (Mill.) } \\
\text { Fuss }\end{array}$ & ERL-72 & Perejil & 2 & & 0 & & 0 & & 0 & \\
\hline 61 & Apiaceae & & RLF-165 & & 1 & & 0 & & 0 & & 0 & \\
\hline
\end{tabular}


Table 5 Species, Spanish common names, number of uses, percentage of families that consume it; cognitive prominence values expressed as $S=$ Sutrop relative prominence index ${ }^{2}$ and biocultural importance expressed as first component value of the principal component analysis by use type (edible, medicinal, firewood, fodder, ceremonial and ornamental; distribution on vegetal types, importance ecological index value (EIVI); specie origin region, ecological status, management practices and management site with respect to species wild populations (Continued)

\begin{tabular}{|c|c|c|c|c|c|c|c|c|c|c|c|c|}
\hline 75 & Apocynaceae & Asclepias curassavica L. & ERL-242 & & 1 & Ornamental $=6$ & 0 & & 0 & 0.0738 & 0 & \\
\hline 76 & Apocynaceae & Asclepias linaria Cav. & RLF-35, SRL-131 & Romero cimarrón & 1 & & 0 & & 0 & -2.1063 & 0 & \\
\hline 64 & Apocynaceae & $\begin{array}{l}\text { Cascabela thevetia (L.) } \\
\text { Lippold }\end{array}$ & SRL-1336 & & 1 & & 0 & & 0 & & 0 & -1.0487 \\
\hline 78 & Apocynaceae & $\begin{array}{l}\text { Funastrum elegans (Decne.) } \\
\text { Schltr. }\end{array}$ & SRL-443, SRL-1153, SRL-1544 & & 1 & & 0 & -1.0765 & 0 & & 0 & \\
\hline 79 & Apocynaceae & $\begin{array}{l}\text { Huernia macrocarpa } \\
\text { Schweinf. ex K.Schum. }\end{array}$ & Photo record & Órgano de Tehuacán & 1 & Ornamental $=6$ & 0 & & 0 & & 0 & \\
\hline 77 & Apocynaceae & Matelea purpusii Woodson & SRL-1123 & Tecacholo & 2 & & 0 & & 0 & & 0.0148 & 0.087 \\
\hline 80 & Apocynaceae & Metastelma sp. & RLF-321 & & & & 0 & & 0 & & 0 & \\
\hline 62 & Apocynaceae & Nerium oleander $\mathrm{L}$. & ERL-103, ERL-123, SRL-178 & Adelfa, laurel & 2 & $\begin{array}{l}\text { Ornamental }=35, \\
\text { ceremonial }=14\end{array}$ & 0 & & 0 & & 0 & \\
\hline 63 & Apocynaceae & Plumeria rubra L. & Photo record & Cacalosuchil & 2 & Ornamental $=12$ & 0 & & 0.0156 & 1.0147 & 0 & \\
\hline 81 & Apocynaceae & & SRL-397 & Tecacholo corriente & & & 0 & & 0 & & 0 & \\
\hline 65 & Araceae & $\begin{array}{l}\text { Zantedeschia aethiopica (L.) } \\
\text { Spreng. }\end{array}$ & SRL-220, ERL-203 & Cartucho & 2 & $\begin{array}{l}\text { Ornamental }=53 \\
\text { ceremonial }=17\end{array}$ & 0 & & 0.0252 & & 0 & \\
\hline 66 & Araliaceae & Aralia humilis Cav. & SRL-1482, SRL-1507 & Mata gallina & 3 & Ornamental $=6$ & 0 & & 0 & -0.3989 & 0 & \\
\hline 67 & Araliaceae & Schefflera sp. & Photo record & & 1 & Ornamental $=6$ & 0 & & 0 & & 0 & \\
\hline 68 & Arecaceae & Brahea dulcis (Kunth) Mart. & $\begin{array}{l}\text { RLF-155, RLF-191,SRL-462, SRL- } \\
\text { 463, SRL-1192, SRL-1193 }\end{array}$ & Palma criolla & 11 & $\begin{array}{l}\text { Ornamental = 35, } \\
\text { ceremonial = 1, } \\
\text { firewood = 100, } \\
\text { 1 ornamental = } 95\end{array}$ & 0.0092 & 7.1968 & 0.0241 & 6.7574 & 0.0035 & 4.3551 \\
\hline 69 & Arecaceae & $\begin{array}{l}\text { Brahea dulcis } \times \text { B. calcarea } \\
\text { Mart. } \times \text { Liebm. }\end{array}$ & SRL-1229 & Palma media sierra & 6 & 1 Ornamental = 95 & 0 & & 0.0049 & 0.1754 & 0 & \\
\hline 70 & Arecaceae & Brahea calcarea Liebm. & SRL-219, SRL-461, SRL-1194 & Palma blanca & 4 & & 0 & & 0.0042 & 0.8205 & 0 & \\
\hline 71 & Arecaceae & Phoenix canariensis Chabaud & Photo record & Palma & 2 & $\begin{array}{l}\text { Ornamental }=18, \\
\text { ceremonial }=1\end{array}$ & 0 & & 0 & & 0 & \\
\hline 72 & Arecaceae & $\begin{array}{l}\text { Washingtonia filifera (Linden } \\
\text { ex André) H.Wendl. ex de } \\
\text { Bary }\end{array}$ & Photo record & Palma & 1 & Ornamental $=12$ & 0 & & 0 & & 0 & \\
\hline 73 & Arecaceae & & ERL-50 & Palmera & 1 & Ornamental $=6$ & 0 & & 0 & & 0 & \\
\hline 74 & Aristolochiaceae & Aristolochia teretiflora Pfeifer & SRL-1130 & Orejita de ratón & 2 & & 0 & & 0 & & 0.0123 & -0.364 \\
\hline 6 & Asparagaceae & Agave americana L. & Photo record & $\begin{array}{l}\text { Maguey de pulque, } \\
\text { Maguey de listón }\end{array}$ & 4 & $\begin{array}{l}\text { Ornamental }=47 \\
18=30\end{array}$ & 0 & & 0 & & 0.0038 & \\
\hline 9 & Asparagaceae & $\begin{array}{l}\text { Agave applanata Lem. ex } \\
\text { Jacobi }\end{array}$ & Photo record & Maguey cenizo & 1 & & 0 & & 0 & & 0 & \\
\hline
\end{tabular}


Table 5 Species, Spanish common names, number of uses, percentage of families that consume it; cognitive prominence values expressed as $S=$ Sutrop relative prominence index ${ }^{2}$ and biocultural importance expressed as first component value of the principal component analysis by use type (edible, medicinal, firewood, fodder, ceremonial and ornamental; distribution on vegetal types, importance ecological index value (EIVI); specie origin region, ecological status, management practices and management site with respect to species wild populations (Continued)

\begin{tabular}{|c|c|c|c|c|c|c|c|c|c|c|c|c|}
\hline 10 & Asparagaceae & Agave kerchovei Lem. & Photo record & Maguey rabo de león & 3 & Edible $=20$ & 0.0020 & -0.2532 & 0 & & 0 & \\
\hline 11 & Asparagaceae & Agave potatorum Zucc. & RLF-285, SRL-403, SRL-1209 & Maguey papalomé & 8 & $\begin{array}{l}\text { Fodder }=5 \\
\text { ornamental }=29, \\
\text { medicinal }=5 \\
\text { edible }=25,18=20\end{array}$ & 0.0068 & 6.6941 & 0.046 & 5.3787 & 0.0388 & 5.4489 \\
\hline 12 & Asparagaceae & $\begin{array}{l}\text { Agave salmiana Otto ex } \\
\text { Salm-Dyck subsp. } \\
\text { tehuacanensis } \\
\text { (Karw. ex Salm-Dyck) García- } \\
\text { Mend. }\end{array}$ & Photo record & Maguey cimarrón & 10 & Ornamental = 12 & 0.0022 & 6.3299 & 0.0098 & 3.672 & 0.0085 & 4.315 \\
\hline 13 & Asparagaceae & Agave scaposa Gentry & Photo record & Maguey potrero & 3 & & 0 & & 0 & & 0.0074 & 2.0018 \\
\hline 14 & Asparagaceae & Agave stricta Salm-Dyck & SRL-1520 & & 1 & & 0 & & 0 & -0.0825 & 0 & \\
\hline 15 & Asparagaceae & Agave titanota Gentry & SRL-404 & Maguey tieso & 2 & & 0 & -0.6097 & 0 & & 0 & \\
\hline 16 & Asparagaceae & Agave triangularis Jacobi & SRL-437 & $\begin{array}{l}\text { Maguey rabo de león, } \\
\text { maguey tieso }\end{array}$ & 3 & & 0 & -0.2987 & 0 & & 0 & \\
\hline 17 & Asparagaceae & Agave tequilana F.A.C.Weber & Photo record & Agave azul & 1 & Ornamental $=6$ & 0 & & 0 & & 0 & \\
\hline 8 & Asparagaceae & Agave vivipara $\mathrm{L}$. & SRL-235, SRL-1353, SRL-1389 & Maguey espadín & 5 & Ornamental = 6 & 0 & & 0.0147 & 1.6977 & 0.0021 & 2.4585 \\
\hline 553 & Asparagaceae & Beaucarnea stricta Lem. & RLF-149 & Sotol & 2 & Ceremonial $=1$ & 0 & & 0 & & 0 & \\
\hline 554 & Asparagaceae & $\begin{array}{l}\text { Dasylirion serratifolium (Karw. } \\
\text { ex Schult. \& Schult.f.) Zucc. }\end{array}$ & $\begin{array}{l}\text { RLF-156, SRL-420, SRL-1473, } \\
\text { SRL-1521 }\end{array}$ & Cucharilla, manita & 5 & $\begin{array}{l}\text { Edible }=95 \\
\text { ceremonial }=5\end{array}$ & 0.0019 & 0.3359 & 0 & & 0 & \\
\hline 50 & Asparagaceae & Echeandia paniculata Rose & SRL-442, SRL-1114 & Cebolla de cacalote & 3 & & 0 & & 0 & & 0 & -0.6167 \\
\hline 51 & Asparagaceae & Echeandia sp. & SRL-319 & Pasto & 1 & & 0 & -1.0765 & 0 & & 0 & \\
\hline 25 & Asparagaceae & Milla biflora Cav. & SRL-1537 & Huele de noche & & & 0 & & 0 & & 0 & \\
\hline 555 & Asparagaceae & $\begin{array}{l}\text { Nolina longifolia (Karw. ex } \\
\text { Schult. \& Schult.f.) Hemsl. }\end{array}$ & SRL-228 & Sotol & 3 & & 0 & & 0 & & 0 & \\
\hline 19 & Asparagaceae & Yucca periculosa Baker & SRL-1505 & Tohuizote & 4 & & 0 & & 0 & & 0 & \\
\hline 18 & Asparagaceae & Yucca gigantea Lem. & SRL-1532 & Huizote, pita, tehuizote & 2 & Ornamental = 12 & 0 & & 0 & & 0 & \\
\hline 215 & Balsaminaceae & Impatiens walleriana Hook.f. & Photo record & Belén & 1 & Ornamental = 12 & 0 & & 0 & & 0 & \\
\hline 216 & Basellaceae & $\begin{array}{l}\text { Anredera cordifolia (Ten.) } \\
\text { Steenis }\end{array}$ & ERL-119 & & 1 & & 0 & & 0 & & 0 & \\
\hline 217 & Berberidaceae & $\begin{array}{l}\text { Berberis pallida Hartw. ex } \\
\text { Benth. }\end{array}$ & $\begin{array}{l}\text { SRL-216, SRL-217, SRL-401, SRL- } \\
\text { 1235, SRL-1399, SRL-1449 }\end{array}$ & Palo tostado & 2 & Firewood $=100$ & 0 & -0.5351 & 0 & & 0 & \\
\hline 218 & Berberidaceae & Berberis sp. & SRL-1428 & & & & 0 & & 0 & & 0 & \\
\hline 219 & Bignoniaceae & $\begin{array}{l}\text { Jacaranda mimosifolia } \\
\text { D.Don }\end{array}$ & ERL-226 & Jacaranda & 3 & Ornamental = 12 & 0 & & 0.0074 & & 0 & \\
\hline
\end{tabular}


Table 5 Species, Spanish common names, number of uses, percentage of families that consume it; cognitive prominence values expressed as $S=$ Sutrop relative prominence index ${ }^{2}$ and biocultural importance expressed as first component value of the principal component analysis by use type (edible, medicinal, firewood, fodder, ceremonial and ornamental; distribution on vegetal types, importance ecological index value (EIVI); specie origin region, ecological status, management practices and management site with respect to species wild populations (Continued)

\begin{tabular}{|c|c|c|c|c|c|c|c|c|c|c|c|c|}
\hline 220 & Bignoniaceae & $\begin{array}{l}\text { Podranea ricasoliana } \\
\text { (Tanfani) Sprague }\end{array}$ & ERL-252 & Flor tronador & 2 & Ornamental = 6 & 0 & & 0 & & 0 & \\
\hline 221 & Bignoniaceae & $\begin{array}{l}\text { Tecoma stans (L.) Juss. ex } \\
\text { Kunth }\end{array}$ & $\begin{array}{l}\text { RLF-13, RLF-56, RLF-249, SRL- } \\
\text { 438, SRL-465, SRL-1307 }\end{array}$ & Tronadora & 2 & & 0 & -0.3922 & 0 & & 0.0013 & -0.6459 \\
\hline 222 & Boraginaceae & $\begin{array}{l}\text { Antiphytum caespitosum } \\
\text { I.M.Johnst. }\end{array}$ & $\begin{array}{l}\text { RLF-125, SRL-99, SRL-1400, SRL- } \\
1466\end{array}$ & Semonilla & 1 & Medicinal $=10$ & 0 & & 0 & & 0.0317 & 0.3143 \\
\hline 223 & Boraginaceae & Borago officinalis $\mathrm{L}$. & SRL-52 & Gordolobo & 1 & & 0 & & 0 & & 0.0046 & \\
\hline 224 & Boraginaceae & $\begin{array}{l}\text { Cordia curassavica (Jacq.) } \\
\text { Roem. \& Schult. }\end{array}$ & SRL-1392 & & 1 & & 0 & -1.0765 & 0 & & 0 & \\
\hline 401 & Boraginaceae & $\begin{array}{l}\text { Nama dichotoma (Ruiz \& } \\
\text { Pav.) Choisy }\end{array}$ & SRL-98, SRL-1182 & & 1 & & 0 & -1.0765 & 0 & & 0 & \\
\hline 402 & Boraginaceae & Nama sp. & SRL-166 & & & & 0 & & 0 & & 0 & \\
\hline 403 & Boraginaceae & $\begin{array}{l}\text { Wigandia urens (Ruiz \& Pav.) } \\
\text { Kunth }\end{array}$ & SRL-1352 & $\begin{array}{l}\text { Chichicasle de tierra } \\
\text { caliente }\end{array}$ & & & 0 & & 0 & & 0 & \\
\hline 225 & Brassicaceae & Brassica oleracea L. & Photo record & Brócoli, Col & 1 & & 0 & & 0 & & 0 & \\
\hline 226 & Brassicaceae & Brassica rapa L. & SRL-1536 & Mostaza & 2 & & 0.0065 & & 0 & & 0 & \\
\hline 229 & Brassicaceae & $\begin{array}{l}\text { Capsella bursa-pastoris (L.) } \\
\text { Medik. }\end{array}$ & SRI-182, SRL-1324 & Lentejilla & 1 & & 0 & & 0 & & 0 & \\
\hline 230 & Brassicaceae & $\begin{array}{l}\text { Descurainia virletii (E.Fourn.) } \\
\text { O.E.Schulz }\end{array}$ & SRL-35 & Mostaza & 2 & & 0 & -0.6097 & 0 & & 0 & \\
\hline 227 & Brassicaceae & Eruca vesicaria (L.) Cav. & RLF-309, SRL-39, SRL-1131 & Jaramón & 2 & Fodder $=40$ & 0.0323 & & 0 & & 0 & \\
\hline 231 & Brassicaceae & Lepidium virginicum L. & $\begin{array}{l}\text { ERL-109, RLF-70, RLF-103, RLF- } \\
179, \text { SRL-1320 }\end{array}$ & Lentejilla & 3 & Ornamental = 35 & 0 & 0.6404 & 0 & 0.4307 & 0.0097 & 0.2534 \\
\hline 232 & Brassicaceae & Matthiola incana (L.) R.Br. & ERL-20, ERL-170 & Alelía & 2 & $\begin{array}{l}\text { Ornamental }=18, \\
\text { ceremonial }=10\end{array}$ & 0 & & 0.0042 & & 0 & \\
\hline 234 & Brassicaceae & Nasturtium officinale R.Br. & SRL-199 & Berro & 2 & Edible $=15$ & 0 & & 0 & & 0 & \\
\hline 233 & Brassicaceae & Raphanus sativus L. & $\begin{array}{l}\text { SRL-44, ERL-136, ERL-162, ERL- } \\
179\end{array}$ & Rábano & 2 & & 0 & & 0 & & 0 & \\
\hline 235 & Brassicaceae & & SRL-1319 & & & & 0 & & 0 & & 0 & \\
\hline 236 & Bromeliaceae & Ananas comosus (L.) Merr. & Photo record & Piña & 2 & Edible $=10$ & 0 & & 0 & & 0.0014 & \\
\hline 237 & Bromeliaceae & Catopsis compacta Mez & RLF-335, SRL1253 & Soluche de jarrita & 5 & $\begin{array}{l}\text { Ornamental = 6, } \\
\text { ceremonial = } 22\end{array}$ & 0 & 3.5020 & 0 & 1.1246 & 0 & 2.2591 \\
\hline 238 & Bromeliaceae & $\begin{array}{l}\text { Hechtia oaxacana Burt-Utley, } \\
\text { Utley \& García-Mend. }\end{array}$ & SRL-405, SRL-1524 & Lechugilla & 1 & Fodder $=10$ & 0.0384 & -0.866 & 0 & & 0 & \\
\hline 239 & Bromeliaceae & Hechtia sp. & SRL-1393 & $\begin{array}{l}\text { Lechugilla de terreno } \\
\text { caliente }\end{array}$ & & & 0 & & 0 & & 0 & \\
\hline
\end{tabular}


Table 5 Species, Spanish common names, number of uses, percentage of families that consume it; cognitive prominence values expressed as $S=$ Sutrop relative prominence index ${ }^{2}$ and biocultural importance expressed as first component value of the principal component analysis by use type (edible, medicinal, firewood, fodder, ceremonial and ornamental; distribution on vegetal types, importance ecological index value (EIVI); specie origin region, ecological status, management practices and management site with respect to species wild populations (Continued)

\begin{tabular}{|c|c|c|c|c|c|c|c|c|c|c|c|c|}
\hline 240 & Bromeliaceae & $\begin{array}{l}\text { Tillandsia acyrostachys } \\
\text { E.Morren ex Baker }\end{array}$ & SRL-1492 & & 2 & Ceremonial $=2$ & 0 & & 0.0221 & -0.4059 & 0 & \\
\hline 241 & Bromeliaceae & Tillandsia bourgaei Baker & SRL-1197 & Soluche blanco & 3 & & 0 & -0.3766 & 0.0221 & -0.5262 & 0 & \\
\hline 242 & Bromeliaceae & Tillandsia grandis Schltdl. & SRL-1472 & Jarrilla & 3 & $\begin{array}{l}\text { Ornamental }=6, \\
\text { ceremonial }=5\end{array}$ & 0.0290 & 2.8484 & 0.0027 & 0.6724 & 0 & \\
\hline 243 & Bromeliaceae & Tillandsia gymnobotrya Baker & SRL-1201, SRL-1435 & $\begin{array}{l}\text { Soluche blanco, } \\
\text { soluche de flor } \\
\text { colorada }\end{array}$ & 5 & $\begin{array}{l}\text { Fodder }=30 \\
\text { ceremonial }=2\end{array}$ & 0.0827 & 0.2377 & 0.0221 & -0.0008 & 0 & -0.2934 \\
\hline 244 & Bromeliaceae & $\begin{array}{l}\text { Tillandsia juncea (Ruiz \& Pav.) } \\
\text { Poir. }\end{array}$ & RLF-81, SRL-1246, SRL-1254 & Soluche & 3 & Ceremonial = 2 & 0 & -0.3767 & 0.0221 & -0.5262 & 0 & \\
\hline 245 & Bromeliaceae & $\begin{array}{l}\text { Tillandsia macdougallii } \\
\text { L.B.Sm. }\end{array}$ & $\begin{array}{l}\text { RLF-84, SRL-224, SRL-1242, SRL- } \\
1250\end{array}$ & Soluche & 3 & Ornamental $=6$ & 0 & 2.8801 & 0.0221 & 1.203 & 0 & \\
\hline 246 & Bromeliaceae & Tillandsia recurvata (L.) L. & SRL-211 & Soluchito & 3 & Ornamental $=6$ & 0.0081 & -0.0731 & 0.0221 & -0.1783 & 0 & -0.4357 \\
\hline 247 & Bromeliaceae & Tillandsia usneoides (L.) L. & SRL-138, SRL-1245 & Apasle & 5 & $\begin{array}{l}\text { Ornamental }=29, \\
\text { ceremonial }=2\end{array}$ & 0.0144 & 4.4242 & 0.009 & 2.5721 & 0 & \\
\hline 248 & Bromeliaceae & Tillandsia sp. & SRL-1244 & Soluche & 2 & Ornamental $=6$ & 0 & 2.5691 & 0.0221 & 0.9401 & 0 & \\
\hline 249 & Bromeliaceae & Tillandsia sp. & SRL-1252 & $\begin{array}{l}\text { Soluche cimarrón, } \\
\text { soluche ixtludo }\end{array}$ & 3 & Ceremonial $=2$ & 0 & 2.879 & 0.0221 & 1.0465 & 0 & \\
\hline 250 & Bromeliaceae & Tillandsia sp. & SRL-1243 & Soluche & 4 & Ceremonial = 2 & 0 & -0.0656 & 0.0221 & -0.2635 & 0 & \\
\hline 251 & Buddlejaceae & Buddleja parviflora Kunth & $\begin{array}{l}\text { ERL-197, SRL-371, SRL-1207, } \\
\text { SRL-1522 }\end{array}$ & $\begin{array}{l}\text { Lengua de vaca, } \\
\text { tepozán }\end{array}$ & 3 & Firewood $=100$ & 0 & & 0 & & 0.0025 & -0.1805 \\
\hline 252 & Buddlejaceae & Buddleja sp. & RLF-83, SRL-30 & & 1 & & 0 & -1.0519 & 0 & & 0 & \\
\hline 253 & Buddlejaceae & Buddleja sp. & SRL-118 & & & & 0 & & 0 & & 0 & \\
\hline 254 & Buddlejaceae & Buddleja sp. & RLF-218, RLF-284 & Tepozán & & & 0 & & 0 & & 0 & \\
\hline 255 & Burseraceae & Bursera biflora (Rose) Standl. & RJS-11, RLF-122, SRL-1219 & $\begin{array}{l}\text { Copal colorado, copal } \\
\text { amarillo, copal criollo }\end{array}$ & 7 & Ceremonial = 99 & 0 & & 0 & 1.805 & 0 & 3.1524 \\
\hline 256 & Burseraceae & $\begin{array}{l}\text { Bursera fagaroides (Kunth) } \\
\text { Engl. }\end{array}$ & SRL-349 & Copalillo & 3 & & 0.0046 & -0.2997 & 0 & & 0 & \\
\hline 257 & Burseraceae & Bursera galeottiana Engl. & RLF-323 & Cuajilote & & & 0 & & 0 & & 0 & \\
\hline 258 & Burseraceae & Bursera morelensis Ramírez & SRL-1345 & & & & 0 & & 0 & & 0 & \\
\hline 259 & Burseraceae & $\begin{array}{l}\text { Bursera pontiveteris Rzed., } \\
\text { Calderón \& Medina }\end{array}$ & SRL-1271 & Copalillo blanco & 2 & & 0 & & 0 & & 0 & \\
\hline 260 & Burseraceae & Bursera schlechtendalii Engl. & SRL-1367 & Aceitillo & 2 & & 0.0027 & -0.7685 & 0 & & 0 & -0.9186 \\
\hline 261 & Burseraceae & Bursera submoniliformis Engl. & SRL-1341, SRL-1346 & Copalillo blanco & & & 0 & & 0 & & 0 & \\
\hline 262 & Cactaceae & $\begin{array}{l}\text { Acanthocereus subinermis } \\
\text { Britton \& Rose }\end{array}$ & Photo record & Nopalito de cruz & 1 & & 0 & & 0 & & 0 & \\
\hline
\end{tabular}


Table 5 Species, Spanish common names, number of uses, percentage of families that consume it; cognitive prominence values expressed as $S=$ Sutrop relative prominence index ${ }^{2}$ and biocultural importance expressed as first component value of the principal component analysis by use type (edible, medicinal, firewood, fodder, ceremonial and ornamental; distribution on vegetal types, importance ecological index value (EIVI); specie origin region, ecological status, management practices and management site with respect to species wild populations (Continued)

\begin{tabular}{|c|c|c|c|c|c|c|c|c|c|c|c|c|}
\hline 263 & Cactaceae & $\begin{array}{l}\text { Cephalocereus columna- } \\
\text { trajani (Karw. ex Pfeiff.) } \\
\text { K.Schum. }\end{array}$ & Photo record & $\begin{array}{l}\text { Cardón pachón, } \\
\text { soldadillo }\end{array}$ & 1 & & 0 & & 0 & -0.1783 & 0 & \\
\hline 264 & Cactaceae & $\begin{array}{l}\text { Coryphantha retusa (Pfeiff) } \\
\text { Britton \& Rose }\end{array}$ & Photo record & Bizniaga & 1 & Ornamental $=12$ & 0 & & 0.0074 & 0.3458 & 0 & \\
\hline 265 & Cactaceae & $\begin{array}{l}\text { Escontria chiotilla (A.A.Weber } \\
\text { ex K.Schum.) Rose }\end{array}$ & Photo record & Jiotilla & 1 & & 0 & & 0 & & 0 & \\
\hline 266 & Cactaceae & $\begin{array}{l}\text { Ferocactus macrodiscus } \\
\text { (Mart.) Britton \& Rose }\end{array}$ & SRL-402 & Bizniaga & 3 & Ornamental = 6 & 0.0161 & 2.7969 & 0.0074 & 0.7647 & 0 & \\
\hline 267 & Cactaceae & $\begin{array}{l}\text { Ferocactus recurvus (Mill.) } \\
\text { Borg }\end{array}$ & SRL-1419 & Bizniaga grande & 3 & Ornamental = 12 & 0.0161 & 3.2785 & 0.0074 & 1.2215 & 0 & \\
\hline 268 & Cactaceae & $\begin{array}{l}\text { Hylocereus undatus (Haw.) } \\
\text { Britton \& Rose }\end{array}$ & Photo record & Pitahaya & 2 & Ornamental = 35 & 0 & & 0 & & 0 & \\
\hline 270 & Cactaceae & $\begin{array}{l}\text { Mammillaria carnea Zucc. ex } \\
\text { Pfeiff. }\end{array}$ & SRL-387 & Biznaga & & & 0 & & 0 & & 0 & \\
\hline 271 & Cactaceae & $\begin{array}{l}\text { Mammillaria haageana } \\
\text { Pfeiff. }\end{array}$ & SRL-387, SRL-1480 & Bizniaga chiquita & 2 & $\begin{array}{l}\text { Ornamental }=18, \\
\text { ceremonial }=2\end{array}$ & 0 & & 0.0074 & 0.8648 & 0 & \\
\hline 272 & Cactaceae & $\begin{array}{l}\text { Mammillaria sphacelata } \\
\text { Mart. }\end{array}$ & Photo record & Biznaga & 2 & Ceremonial $=2$ & 0 & & 0.0074 & 0.6323 & 0 & \\
\hline 273 & Cactaceae & Mammillaria sp. & Photo record & Biznaga & 1 & & 0 & & 0.0074 & -1.9051 & 0 & \\
\hline 274 & Cactaceae & Mammillaria sp. & Photo record & Bizniaga & 1 & Ornamental $=6$ & 0 & & 0.0074 & 0.1793 & 0 & \\
\hline 269 & Cactaceae & $\begin{array}{l}\text { Marginatocereus marginatus } \\
\text { (DC.) Backeb. }\end{array}$ & SRL-237 & Órgano & 5 & $\begin{array}{l}\text { Ornamental }=59 \\
\text { ceremonial }=40\end{array}$ & 0 & & 0 & & 0 & \\
\hline 275 & Cactaceae & Opuntia depressa Rose & SRL-238 & Nopal de coyote & 3 & & 0.007 & 0.0052 & 0 & & 0.0139 & -0.1499 \\
\hline 276 & Cactaceae & Opuntia ficus-indica (L.) Mill. & Photo record & $\begin{array}{l}\text { Nopal de castilla, nopal } \\
\text { pelón }\end{array}$ & 2 & Edible $=100$ & 0 & & 0 & & 0 & \\
\hline 277 & Cactaceae & Opuntia huajuapensis Bravo & SRL-239 & Nopal & 3 & & 0.0072 & 1.1617 & 0 & & 0 & \\
\hline 278 & Cactaceae & Opuntia lasiacantha Pfeiff. & SRL-477 & Nopal pachón & 2 & Edible $=100$ & 0 & & 0 & & 0 & \\
\hline 279 & Cactaceae & Opuntia sp. & SRL-236 & Nopal amarillo & 3 & & 0.0072 & & 0 & & 0 & \\
\hline 280 & Cactaceae & Opuntia sp. & Photo record & $\begin{array}{l}\text { Nopal de coyote, } \\
\text { nopal tuna roja }\end{array}$ & 3 & & 0.0072 & 4.1969 & 0 & & 0 & \\
\hline 281 & Cactaceae & Opuntia sp. & Photo record & Nopal de sacristán & 2 & & 0.0072 & 1.0967 & 0 & & 0 & \\
\hline 282 & Cactaceae & $\begin{array}{l}\text { Pachycereus weberi (J.M. } \\
\text { Coult.) Backeb. }\end{array}$ & Photo record & Cardón verde & & & 0 & & 0 & & 0 & \\
\hline 283 & Cactaceae & $\begin{array}{l}\text { Pseudomitrocereus fulviceps } \\
\text { (F.A.C.Weber ex K.Schum.) } \\
\text { Bravo \& Buxb. }\end{array}$ & SRL-1451, SRL-1501 & Cardón & & & 0 & & 0 & & 0 & \\
\hline
\end{tabular}


Table 5 Species, Spanish common names, number of uses, percentage of families that consume it; cognitive prominence values expressed as $S=$ Sutrop relative prominence index ${ }^{2}$ and biocultural importance expressed as first component value of the principal component analysis by use type (edible, medicinal, firewood, fodder, ceremonial and ornamental; distribution on vegetal types, importance ecological index value (EIVI); specie origin region, ecological status, management practices and management site with respect to species wild populations (Continued)

\begin{tabular}{|c|c|c|c|c|c|c|c|c|c|c|c|c|}
\hline 284 & Cactaceae & & SRL-1452 & & & & 0 & & 0 & & 0 & \\
\hline 285 & Calochortaceae & $\begin{array}{l}\text { Calochortus barbatus (Kunth) } \\
\text { J.H.Painter }\end{array}$ & SRL-1204 & & & & 0 & & 0 & & 0 & \\
\hline 286 & Campanulaceae & $\begin{array}{l}\text { Diastatea micrantha (Kunth) } \\
\text { McVaugh }\end{array}$ & SRL-156, SRL-157 & & 2 & & 0 & -0.7655 & 0 & -1.4404 & 0 & \\
\hline 763 & Cannabaceae & Celtis caudata Planch. & $\begin{array}{l}\text { ERL-79, ERL-155, ERL-194, ERL- } \\
\text { 222, SRL-1475 }\end{array}$ & Malintze, moralillo & 5 & & 0.0161 & 1.0117 & 0 & & 0 & \\
\hline 287 & Cannaceae & Canna indica L. & SRL-57, ERL-43, ERL-217 & Platanillo & 2 & $\begin{array}{l}\text { Ornamental }=35 \\
\text { ceremonial }=10\end{array}$ & 0 & & 0 & & 0 & \\
\hline 288 & Capparaceae & Capparis pringlei Briq. & SRL-1354 & & & & 0 & & 0 & & 0 & \\
\hline 213 & Caprifoliaceae & Scabiosa atropurpurea $\mathrm{L}$. & ERL-23, ERL-239 & Barín & 2 & Ornamental $=6$ & 0 & & 0 & & 0 & \\
\hline 767 & Caprifoliaceae & Valeriana sp. & RLF-28, RLF-199, SRL-1300 & & 1 & & 0 & -1.0758 & 0 & & 0 & \\
\hline 289 & Caricaceae & Carica papaya L. & Photo record & Papaya & 1 & Edible $=5$ & 0 & & 0 & & 0 & \\
\hline 290 & Caryophyllaceae & Dianthus caryophyllus L. & Photo record & Clavel & 2 & & 0 & & 0.0118 & & 0 & \\
\hline 291 & Casuarinaceae & Casuarina equisetifolia $\mathrm{L}$. & SRL-41 & Ocote corriente, pino & 4 & Ornamental = 35 & 0 & & 0.0588 & & 0.0015 & \\
\hline 292 & Celastraceae & $\begin{array}{l}\text { Acanthothamnus aphyllus } \\
\text { (Schltdl.) Standl. }\end{array}$ & SRL-1504 & & & & 0 & & 0 & & 0 & \\
\hline 293 & Celastraceae & Cassine xylocarpa Vent. & SRL-1334 & & & & 0 & & 0 & & 0 & \\
\hline 294 & Chenopodiaceae & $\begin{array}{l}\text { Chenopodium berlandieri } \\
\text { Moq. }\end{array}$ & SRL-1139 & $\begin{array}{l}\text { Quelite de manteca, } \\
\text { flor de huizontle }\end{array}$ & 2 & Edible $=15$ & 0.0046 & -0.3023 & 0 & & 0 & \\
\hline 295 & Chenopodiaceae & Chenopodium murale L. & $\begin{array}{l}\text { RLF-184, SRL-194, SRL-1121, } \\
\text { SRL-1140, SRL-1321 }\end{array}$ & Quelite de guajolote & 3 & $\begin{array}{l}\text { Fodder }=10 \\
\text { medicinal }=40\end{array}$ & 0.0054 & & 0 & & 0 & \\
\hline 296 & Chenopodiaceae & $\begin{array}{l}\text { Dysphania ambrosioides (L.) } \\
\text { Mosyakin \& Clemants }\end{array}$ & $\begin{array}{l}\text { ERL-32, ERL-33, ERL-168, RLF-89, } \\
\text { SRL-1136 }\end{array}$ & Epazote & 4 & Edible $=100$ & 0 & & 0 & & 0.0123 & 1.3678 \\
\hline 297 & Chenopodiaceae & Spinacia oleracea L. & Photo record & Espinaca & 1 & & 0 & & 0 & & 0 & \\
\hline 298 & Cistaceae & Helianthemum sp. & RLF-17 & & & & 0 & & 0 & & 0 & \\
\hline 299 & Commelinaceae & Commelina erecta $\mathrm{L}$. & RLF-19, RLF-73, SRL-159 & & 2 & & 0 & -0.6240 & 0 & -1.3004 & 0 & \\
\hline 300 & Commelinaceae & Commelina sp. & SRL-48 & & 1 & & 0 & & 0 & & 0 & \\
\hline 301 & Commelinaceae & Gibasis consobrina D.R.Hunt & RLF-190, SRL-430 & $\begin{array}{l}\text { Milpa, lengua de } \\
\text { cucho }\end{array}$ & & & 0 & & 0 & & 0 & \\
\hline 302 & Commelinaceae & $\begin{array}{l}\text { Gibasis geniculata (Jacq.) } \\
\text { Rohweder }\end{array}$ & ERL-44 & & 1 & Ornamental $=6$ & 0 & & 0 & & 0 & \\
\hline 303 & Commelinaceae & Tradescantia crassifolia Cav. & SRL-149 & & 1 & & 0 & & 0 & & 0 & -1.0487 \\
\hline 304 & Commelinaceae & $\begin{array}{l}\text { Tripogandra purpurascens } \\
\text { (S.Schauer) Handlos }\end{array}$ & RLF-15 & & 1 & & 0 & -0.8960 & 0 & & 0 & \\
\hline
\end{tabular}


Table 5 Species, Spanish common names, number of uses, percentage of families that consume it; cognitive prominence values expressed as $\mathrm{S}=$ Sutrop relative prominence index ${ }^{2}$ and biocultural importance expressed as first component value of the principal component analysis by use type (edible, medicinal, firewood, fodder, ceremonial and ornamental; distribution on vegetal types, importance ecological index value (EIVI); specie origin region, ecological status, management practices and management site with respect to species wild populations (Continued)

\begin{tabular}{|c|c|c|c|c|c|c|c|c|c|c|c|c|}
\hline 84 & Compositae & $\begin{array}{l}\text { Acourtia scapiformis (Bacig.) } \\
\text { B.L.Turner }\end{array}$ & SRL-163 & & 1 & & 0 & & 0 & -2.1063 & 0 & \\
\hline 85 & Compositae & Acourtia sp. & SRL-215,SRL-1468 & Oreja de conejo & & & 0 & & 0 & & 0 & \\
\hline 86 & Compositae & $\begin{array}{l}\text { Adenophyllum glandulosum } \\
\text { (Cav.) Strother }\end{array}$ & SRL-1264 & & 1 & & 0 & -1.0766 & 0 & & 0 & \\
\hline 87 & Compositae & $\begin{array}{l}\text { Ageratina espinosarum } \\
\text { (A.Gray) R.M.King \& H.Rob. }\end{array}$ & $\begin{array}{l}\text { RLF-36, SRL-114, SRL-291, SRL- } \\
\text { 325, SRL-363, SRL-1279 }\end{array}$ & & 2 & & 0 & 0.0391 & 0 & & 0 & -0.347 \\
\hline 88 & Compositae & $\begin{array}{l}\text { Ageratina mairetiana (DC.) } \\
\text { R.M.King \& H.Rob. }\end{array}$ & SRL-186, SRL-390 & Hierba de ángel & 3 & $\begin{array}{l}\text { Ornamental }=6 \\
\text { medicinal }=15\end{array}$ & 0 & 3.3978 & 0 & 0.9653 & 0.15 & 5.7983 \\
\hline 89 & Compositae & $\begin{array}{l}\text { Ageratina tomentella } \\
\text { (Schrad.) R.M.King \& H.Rob. }\end{array}$ & $\begin{array}{l}\text { RLF-217, SRL-119, SRL-289, SRL- } \\
\text { 335, SRL-391, SRL-1191, SRL- } \\
\text { 1398, SRL-1406 }\end{array}$ & & 1 & & 0 & -0.7561 & 0 & & 0 & \\
\hline 90 & Compositae & Ageratina sp. & RLF-116, SRL-74 & Hierba de ángel & 3 & & 0 & -0.2987 & 0 & -1.0819 & 0 & -0.6123 \\
\hline 91 & Compositae & Ageratina sp. & RLF-4, SRL-153, SRL-287 & Niebla & 2 & & 0 & -0.6843 & 0 & -1.36 & 0 & \\
\hline 92 & Compositae & Ageratina sp. & SRL-208 & Oreganillo & 1 & & 0 & & 0 & & 0 & -0.8137 \\
\hline 93 & Compositae & $\begin{array}{l}\text { Ageratum tehuacanum } \\
\text { R.M.King \& H.Rob. }\end{array}$ & RLF-26, SRL-113 & Cara blanca & 1 & & 0 & -1.0765 & 0 & & 0 & \\
\hline 94 & Compositae & Ambrosia psilostachya DC. & RLF-9 & & 1 & Medicinal $=5$ & 0 & & 0 & & 0 & -0.5778 \\
\hline 97 & Compositae & $\begin{array}{l}\text { Archibaccharis serratifolia } \\
\text { (Kunth) S. F. Blake }\end{array}$ & $\begin{array}{l}\text { RLF-257, SRL-267, SRL-292, SRL- } \\
1241\end{array}$ & & 1 & & 0 & -0.9975 & 0 & & 0 & \\
\hline 95 & Compositae & Artemisia ludoviciana Nutt. & Photo record & $\begin{array}{l}\text { Ajenjo, estafiate, hierba } \\
\text { maistra }\end{array}$ & 1 & Medicinal $=10$ & 0 & & 0 & & 0.0591 & \\
\hline 214 & Compositae & Baccharis conferta Kunth & Photo record & & 1 & & 0 & & 0 & & 0 & \\
\hline 98 & Compositae & $\begin{array}{l}\text { Baccharis salicina Torr. \& } \\
\text { A.Gray }\end{array}$ & SRL-1151 & Chamizo & 1 & & 0 & & 0 & & 0 & -1.0487 \\
\hline 99 & Compositae & $\begin{array}{l}\text { Barkleyanthus salicifolius } \\
\text { (Kunth) H.Rob. \& Brettell }\end{array}$ & $\begin{array}{l}\text { SRL-190, SRL-1531, ERL-27, ERL- } \\
\text { 83, ERL-190, ERL-218 }\end{array}$ & Somiate & 6 & $\begin{array}{l}\text { Ornamental =65, } \\
\text { firewood }=100\end{array}$ & 0.0323 & 0.9183 & 0 & 1.6175 & 0.0291 & 0.6711 \\
\hline 100 & Compositae & Bidens bigelovii A.Gray & RLF-140, RLF-196 & Cahual cimarrón & 1 & & 0 & -1.0765 & 0 & & 0 & \\
\hline 101 & Compositae & Bidens pilosa L. & SRL-4, SRL-1285 & Oaxaqueña & 2 & Fodder $=40$ & 0 & -0.0737 & 0 & & 0 & -0.485 \\
\hline 102 & Compositae & Bidens sp. & $\begin{array}{l}\text { RLF-221, SRL-316, SRL-395, SRL- } \\
1288\end{array}$ & & 1 & & 0 & -0.6451 & 0 & & 0 & \\
\hline 103 & Compositae & $\begin{array}{l}\text { Brickellia veronicifolia (Kunth) } \\
\text { A.Gray }\end{array}$ & $\begin{array}{l}\text { RLF-11, RLF-203, RLF-206, SRL- } \\
\text { 293, SRL-361, SRL-1276, ERL-101 }\end{array}$ & $\begin{array}{l}\text { Oreganillo, orejita de } \\
\text { ratón }\end{array}$ & 3 & & 0 & 0.4263 & 0 & & 0 & -0.1215 \\
\hline 104 & Compositae & Brickellia sp. & SRL-1418 & & 1 & & 0 & -1.0765 & 0 & & 0 & \\
\hline 105 & Compositae & Calendula officinalis $\mathrm{L}$. & SRL-49, ERL-22, ERL-24 & Mercader amarillo & 3 & Ornamental $=18$ & 0 & & 0 & & 0 & \\
\hline
\end{tabular}


Table 5 Species, Spanish common names, number of uses, percentage of families that consume it; cognitive prominence values expressed as $\mathrm{S}=$ Sutrop relative prominence index ${ }^{2}$ and biocultural importance expressed as first component value of the principal component analysis by use type (edible, medicinal, firewood, fodder, ceremonial and ornamental; distribution on vegetal types, importance ecological index value (EIVI); specie origin region, ecological status, management practices and management site with respect to species wild populations (Continued)

\begin{tabular}{|c|c|c|c|c|c|c|c|c|c|c|c|c|}
\hline 106 & Compositae & $\begin{array}{l}\text { Carminatia alvarezii Rzed. \& } \\
\text { Calderón }\end{array}$ & RLF-186, SRL-127, SRL-1308 & Oaxaqueña & 1 & & 0 & -1.0765 & 0 & & 0 & \\
\hline 107 & Compositae & Chrysactinia mexicana A.Gray & RLF-154, SRL-1163 & Hierba de San Nicolás & 1 & & 0 & & 0 & & 0.0167 & -0.3058 \\
\hline 109 & Compositae & $\begin{array}{l}\text { Chrysanthemum morifolium } \\
\text { Ramat. }\end{array}$ & ERL-143, ERL-163, ERL-230 & $\begin{array}{l}\text { Cresentena, nora, } \\
\text { teresita }\end{array}$ & 2 & $\begin{array}{l}\text { Ornamental }=53 \\
\text { ceremonial }=29\end{array}$ & 0 & & 0 & & 0 & \\
\hline 110 & Compositae & Cirsium mexicanum DC. & SRL-435 & Lechuga cimarrón & 2 & & 0 & -0.6097 & 0 & & 0 & \\
\hline 111 & Compositae & Cirsium sp. & SRL-400, SRL-1427 & $\begin{array}{l}\text { Espino del diablo, } \\
\text { chicalote de monte }\end{array}$ & 1 & & 0 & -1.0765 & 0 & & 0 & \\
\hline 112 & Compositae & Coreopsis sp. & SRL-314 & & 1 & & 0 & 0.0527 & 0 & & 0 & \\
\hline 113 & Compositae & Cosmos bipinnatus Cav. & $\begin{array}{l}\text { ERL-4, ERL-166, ERL-167, SRL-45, } \\
\text { SRL-47 }\end{array}$ & Jazmín & 2 & $\begin{array}{l}\text { Ornamental }=24, \\
\text { ceremonial }=8\end{array}$ & 0 & & 0 & & 0 & \\
\hline 114 & Compositae & $\begin{array}{l}\text { Dahlia apiculata (Sherff) } \\
\text { P.D.Sorensen }\end{array}$ & $\begin{array}{l}\text { RLF-259, SRL-91, SRL-1199, ERL- } \\
\text { 133, ERL-148 }\end{array}$ & $\begin{array}{l}\text { Dalia corriente, } \\
\text { ticurrichi }\end{array}$ & 2 & Ornamental $=12$ & 0 & & 0 & 1.0674 & 0 & \\
\hline 115 & Compositae & Dahlia coccinea Cav. & $\begin{array}{l}\text { RLF-96, RLF-260, SRL-423, SRL- } \\
\text { 1160, SRL-1186 }\end{array}$ & Dalia & 2 & & 0 & & 0 & 0.7547 & 0 & \\
\hline 116 & Compositae & Dahlia sp. & $\begin{array}{l}\text { ERL-132, ERL-251, ERL-253, ERL- } \\
452\end{array}$ & Dalia & 2 & Ceremonial $=8$ & 0 & & 0.015 & & 0 & \\
\hline 117 & Compositae & Desmanthodium sp. & SRL-270 & & & & 0 & & 0 & & 0 & \\
\hline 118 & Compositae & $\begin{array}{l}\text { Dyssodia papposa (Vent.) } \\
\text { Hitchc. }\end{array}$ & $\begin{array}{l}\text { RLF-240, SRL-5, SRL-410, SRL- } \\
1290\end{array}$ & Cempasuchito & 1 & & 0 & -1.0765 & 0 & & 0 & \\
\hline 119 & Compositae & Dyssodia sp. & RLF-24, SRL-121, SRL-379 & & 1 & & 0 & & 0 & -2.1063 & 0 & \\
\hline 120 & Compositae & Erigeron karvinskianus DC. & RLF-270 & & 1 & & 0 & -1.0765 & 0 & & 0 & \\
\hline 121 & Compositae & Erigeron sp. & SRL-409 & & & & 0 & & 0 & & 0 & \\
\hline 122 & Compositae & $\begin{array}{l}\text { Flaveria trinervia (Spreng.) } \\
\text { C.Mohr }\end{array}$ & SRL-16 & Romero cimarrón & & & 0 & & 0 & & 0 & \\
\hline 123 & Compositae & Galinsoga parviflora Cav. & RLF-269, SRL-1176 & & 1 & & 0 & -1.0765 & 0 & & 0 & \\
\hline 108 & Compositae & $\begin{array}{l}\text { Glebionis coronaria (L.) Cass. } \\
\text { ex Spach }\end{array}$ & $\begin{array}{l}\text { SRL-10, ERL-16, ERL-17, ERL-34, } \\
\text { ERL-35, ERL-255, ERL-256 }\end{array}$ & Linda & 2 & $\begin{array}{l}\text { Ornamental }=29 \\
\text { ceremonial }=29\end{array}$ & 0 & & 0.0131 & & 0 & \\
\hline 124 & Compositae & Gnaphalium sp. & RLF-188 & & 1 & & 0 & & 0 & -2.1063 & 0 & \\
\hline 125 & Compositae & Gnaphalium sp. & SRL-297 & & 1 & Medicinal $=5$ & 0 & & 0 & & 0 & -0.6864 \\
\hline 126 & Compositae & $\begin{array}{l}\text { Gochnatia hypoleuca (DC.) } \\
\text { A.Gray }\end{array}$ & SRL-1464 & & & & 0 & & 0 & & 0 & \\
\hline 127 & Compositae & Grindelia inuloides Willd. & $\begin{array}{l}\text { RLF-14, SRL-66, SRL-107, SRL- } \\
\text { 278, SRL-295, SRL-365, SRL-1547 }\end{array}$ & Árnica & 1 & Medicinal $=25$ & 0 & & 0 & & 0.0938 & 5.0025 \\
\hline 128 & Compositae & $\begin{array}{l}\text { Gymnosperma glutinosum } \\
\text { (Spreng.) Less. }\end{array}$ & $\begin{array}{l}\text { RLF-72, RLF-121, SRL-75, SRL- } \\
\text { 290, SRL-1117, SRL-1287, ERL-25 }\end{array}$ & Cerilla, popote & 2 & Medicinal $=5$ & 0.0036 & 0.2317 & 0 & & 0.0309 & 0.6436 \\
\hline
\end{tabular}


Table 5 Species, Spanish common names, number of uses, percentage of families that consume it; cognitive prominence values expressed as $S=$ Sutrop relative prominence index ${ }^{2}$ and biocultural importance expressed as first component value of the principal component analysis by use type (edible, medicinal, firewood, fodder, ceremonial and ornamental; distribution on vegetal types, importance ecological index value (EIVI); specie origin region, ecological status, management practices and management site with respect to species wild populations (Continued)

\begin{tabular}{|c|c|c|c|c|c|c|c|c|c|c|c|c|}
\hline 129 & Compositae & Helenium mexicanum Kunth & RLF-25, SRL-1116, SRL-1134 & Chiche de perro & 2 & & 0 & & 0 & & 0 & -0.8599 \\
\hline 130 & Compositae & Helianthus annuus L. & Photo record & Girasol & 2 & Ornamental $=6$ & 0 & & 0 & & 0 & \\
\hline 132 & Compositae & Lactuca sativa $\mathrm{L}$. & Photo record & Lechuga & 1 & & 0 & & 0 & & 0 & \\
\hline 131 & Compositae & $\begin{array}{l}\text { Launaea intybacea (Jacq.) } \\
\text { Beauverd }\end{array}$ & SRL-69 & Mostaza & 1 & & 0 & & 0 & & 0 & \\
\hline 133 & Compositae & $\begin{array}{l}\text { Leucanthemum maximum } \\
\text { (Ramond) DC. }\end{array}$ & ERL-138 & Margarita, margaritón & 2 & $\begin{array}{l}\text { Ornamental }=24, \\
\text { ceremonial }=8\end{array}$ & 0 & & 0.0095 & & 0 & \\
\hline 134 & Compositae & Matricaria chamomilla L. & SRL-175 & Manzanilla & 1 & Medicinal $=55$ & 0 & & 0 & & 0.0868 & \\
\hline 135 & Compositae & $\begin{array}{l}\text { Melampodium divaricatum } \\
\text { (Rich. ex Rich.) DC. }\end{array}$ & RLF-205 & Chimalacate & 2 & & 0 & -0.7656 & 0 & -1.4404 & 0 & \\
\hline 136 & Compositae & $\begin{array}{l}\text { Melampodium longifolium } \\
\text { Cerv. ex Cav. }\end{array}$ & SRL-129, RLF-261 & & 1 & & 0 & & 0 & -1.5115 & 0 & \\
\hline 137 & Compositae & Melampodium sp. & RLF-220 & & 1 & & 0 & & 0 & -2.1063 & 0 & \\
\hline 138 & Compositae & Montanoa tomentosa Cerv. & RLF-300, SRL-2 & Oaxaqueña & 1 & & 0 & & 0 & & 0 & -1.0367 \\
\hline 139 & Compositae & Montanoa sp. & RLF-299 & & 1 & & 0 & -1.0354 & 0 & & 0 & \\
\hline 150 & Compositae & $\begin{array}{l}\text { Neurolaena lobata (L.) R.Br. } \\
\text { ex Cass. }\end{array}$ & SRL-198 & Naranjillo & 2 & & 0 & & 0 & & 0 & -0.8599 \\
\hline 140 & Compositae & $\begin{array}{l}\text { Parthenium bipinnatifidum } \\
\text { (Ortega) Rollins }\end{array}$ & $\begin{array}{l}\text { ERL-9, RLF-87, RLF-178, SRL-34, } \\
\text { SRL-82, SRL-445, SRL-1325 }\end{array}$ & Hierba cenizo & 2 & & 0 & -0.2194 & 0 & & 0 & -0.507 \\
\hline 141 & Compositae & Parthenium tomentosum DC. & SRL-1213, SRL-1375 & Palo prieto & 2 & & 0 & & 0 & & 0 & -0.86 \\
\hline 142 & Compositae & Parthenium sp. & RLF-198 & & & & 0 & & 0 & & 0 & \\
\hline 143 & Compositae & Perymenium discolor Schrad. & SRL-277, SRL-1266 & & 1 & & 0 & -0.2154 & 0 & & 0 & \\
\hline 144 & Compositae & $\begin{array}{l}\text { Perymenium mendezii var. } \\
\text { angustifolium (Brandegee) } \\
\text { J.J.Fay }\end{array}$ & RLF-110, SRL-351 & Cahual delgado & 1 & & 0 & -1.0332 & 0 & & 0 & \\
\hline 145 & Compositae & Perymenium sp. & RLF-251 & Cahual & 2 & & 0 & -0.6097 & 0 & & 0 & -0.8011 \\
\hline 146 & Compositae & Philactis zinnioides Schrad. & RLF-322 & & & & 0 & & 0 & & 0 & \\
\hline 147 & Compositae & $\begin{array}{l}\text { Pinaropappus roseus (Less.) } \\
\text { Less. }\end{array}$ & RJS-8, SRL-407, SRL-1526 & Chipule & 1 & & 0 & & 0 & & 0.0119 & -0.8163 \\
\hline 148 & Compositae & Piqueria trinervia Cav. & RLF-8 & & 2 & & 0 & -0.6097 & 0 & & 0 & -0.8011 \\
\hline 151 & Compositae & $\begin{array}{l}\text { Porophyllum linaria (Cav.) } \\
\text { DC. }\end{array}$ & $\begin{array}{l}\text { RLF-18, SRL-158, SRL-357, SRL- } \\
\text { 1150, ERL-141 }\end{array}$ & Pepitza & 4 & Edible $=95$ & 0 & & 0.0098 & 2.0349 & 0 & 3.1943 \\
\hline 153 & Compositae & $\begin{array}{l}\text { Porophyllum punctatum } \\
\text { (Mill.) S.F.Blake }\end{array}$ & SRL-207 & Papaloquelite & 1 & & 0 & & 0 & & 0 & \\
\hline
\end{tabular}


Table 5 Species, Spanish common names, number of uses, percentage of families that consume it; cognitive prominence values expressed as $S=$ Sutrop relative prominence index ${ }^{2}$ and biocultural importance expressed as first component value of the principal component analysis by use type (edible, medicinal, firewood, fodder, ceremonial and ornamental; distribution on vegetal types, importance ecological index value (EIVI); specie origin region, ecological status, management practices and management site with respect to species wild populations (Continued)

\begin{tabular}{|c|c|c|c|c|c|c|c|c|c|c|c|c|}
\hline 152 & Compositae & $\begin{array}{l}\text { Porophyllum ruderale subsp. } \\
\text { macrocephalum } \\
\text { (DC.) R.R.Johnson }\end{array}$ & RLF-318, SRL-1539 & Papaloquelite & 2 & Edible $=90$ & 0 & & 0 & & 0 & \\
\hline 154 & Compositae & $\begin{array}{l}\text { Psacalium amplifolium (DC.) } \\
\text { H.Rob. \& Brettell }\end{array}$ & RLF-39, SRL-266 & & & & 0 & & 0 & & 0 & \\
\hline 155 & Compositae & $\begin{array}{l}\text { Psacalium paucicapitatum } \\
\text { (B.L.Rob. \& Greenm.) } \\
\text { H.Rob. \& Brettell }\end{array}$ & RLF-193, SRL-1159 & $\begin{array}{l}\text { Hierba de camote de } \\
\text { venado }\end{array}$ & 1 & & 0 & & 0 & & 0.0069 & 1.6353 \\
\hline 156 & Compositae & Psacalium sp. & RLF-40 & $\begin{array}{l}\text { Malangar chico, hierba } \\
\text { de cruz }\end{array}$ & & & 0 & & 0 & & 0 & \\
\hline 157 & Compositae & $\begin{array}{l}\text { Roldana oaxacana (Hemsl.) } \\
\text { H.Rob. \& Brettell }\end{array}$ & SRL-1411 & & & & 0 & & 0 & & 0 & \\
\hline 189 & Compositae & $\begin{array}{l}\text { Roldana ehrenbergiana } \\
\text { (Klatt) H.Rob. \& Brettell }\end{array}$ & SRL-1152 & Hierba de perro & 2 & & 0 & & 0 & & 0 & -0.8599 \\
\hline 158 & Compositae & Sanvitalia procumbens Lam. & RLF-42, SRL-12, SRL-1179 & Ojo de gallo & 2 & & 0 & -0.1373 & 0 & & 0 & -0.4562 \\
\hline 149 & Compositae & Senecio praecox (Cav.) DC. & ERL-191, SRL-1487 & $\begin{array}{l}\text { Consuelda, pata de } \\
\text { león }\end{array}$ & 3 & Ornamental $=12$ & 0 & & 0.0059 & 0.9165 & 0.0093 & 2.0335 \\
\hline 159 & Compositae & Simsia lagascaeformis A.Gray & $\begin{array}{l}\text { RLF-310, RLF-297, SRL-28, SRL- } \\
37\end{array}$ & $\begin{array}{l}\text { Cahual de burro, } \\
\text { cahual }\end{array}$ & 2 & $\begin{array}{l}\text { Fodder }=80 \\
\text { ornamental }=35\end{array}$ & 0.1501 & 0.2470 & 0 & 0.235 & 0 & \\
\hline 160 & Compositae & Simsia sanguinea A.Gray & RLF-55, SRL-112 & & 1 & & 0 & -1.0765 & 0 & & 0 & \\
\hline 161 & Compositae & Simsia sp. & RLF-80 & Cahual chiquito & 1 & & 0 & -1.0765 & 0 & & 0 & \\
\hline 162 & Compositae & Sonchus oleraceus (L.) L. & ERL-10, SRL-1126 & Chicoria & 1 & & 0 & & 0 & & 0 & \\
\hline 168 & Compositae & Stevia caracasana DC. & $\begin{array}{l}\text { RLF-211, SRL-1289, SRL-1293, } \\
\text { SRL-1402 }\end{array}$ & & 2 & Ceremonial $=8$ & 0 & & 0 & -1.201 & 0 & \\
\hline 163 & Compositae & Stevia lucida Lag. & SRL-332, SRL-339 & Chamalacate & 2 & & 0 & -0.2391 & 0 & & 0 & \\
\hline 164 & Compositae & Stevia serrata Cav. & SRL-298 & & & & 0 & & 0 & & 0 & \\
\hline 165 & Compositae & Stevia sp. & $\begin{array}{l}\text { RLF-2, SRL-282, SRL-288, SRL- } \\
313\end{array}$ & & & & 0 & & 0 & & 0 & \\
\hline 166 & Compositae & Stevia sp. & $\begin{array}{l}\text { RLF-170, RLF-183, SRL-32, SRL- } \\
\text { 97, SRL-1281 }\end{array}$ & Cahual delgado & 2 & & 0 & -0.2980 & 0 & & 0 & -0.5662 \\
\hline 167 & Compositae & Stevia sp. & RLF-276 & Cahual prieto & 1 & & 0 & & 0 & & 0 & -1.0487 \\
\hline 169 & Compositae & Stevia sp. & SRL-1262 & & 2 & & 0 & & 0 & & 0 & -0.8599 \\
\hline 170 & Compositae & Stevia sp. & SRL-1295 & & 2 & & 0 & -0.2980 & 0 & & 0 & -0.5662 \\
\hline 96 & Compositae & $\begin{array}{l}\text { Symphyotrichum novi-belgii } \\
\text { (L.) G.L.Nesom }\end{array}$ & $\begin{array}{l}\text { SRL-56, ERL-66, ERL-86, ERL-154, } \\
\text { ERL-225 }\end{array}$ & Esther & 2 & $\begin{array}{l}\text { Ornamental }=47 \\
\text { ceremonial }=14\end{array}$ & 0 & & 0.0017 & & 0 & \\
\hline
\end{tabular}


Table 5 Species, Spanish common names, number of uses, percentage of families that consume it; cognitive prominence values expressed as $S=$ Sutrop relative prominence index ${ }^{2}$ and biocultural importance expressed as first component value of the principal component analysis by use type (edible, medicinal, firewood, fodder, ceremonial and ornamental; distribution on vegetal types, importance ecological index value (EIVI); specie origin region, ecological status, management practices and management site with respect to species wild populations (Continued)

\begin{tabular}{|c|c|c|c|c|c|c|c|c|c|c|c|c|}
\hline 171 & Compositae & Tagetes erecta $\mathrm{L}$. & $\begin{array}{l}\text { ERL-12, ERL-62, ERL-117, ERL- } \\
\text { 118, ERL-134, ERL-149, ERL-151, } \\
\text { ERL-152, ERL-159, SRL-7, SRL- } \\
408, \text { SRL-1142 }\end{array}$ & Cempasuchí & 3 & $\begin{array}{l}\text { Ornamental }=71 \\
\text { ceremonial }=99\end{array}$ & 0 & & 0.0189 & & 0.0026 & \\
\hline 172 & Compositae & Tagetes lucida Cav. & $\begin{array}{l}\text { RLF-3, SRL-377, SRL-1232, SRL- } \\
1426\end{array}$ & Pericón & 4 & Ceremonial $=50$ & 0 & & 0.0241 & -0.1211 & 0.0523 & 0.4295 \\
\hline 173 & Compositae & Tagetes Iunulata Ortega & ERL-137 & Cempasuchí chiquito & 3 & $\begin{array}{l}\text { Ornamental }=29, \\
\text { ceremonial }=40\end{array}$ & 0.0027 & 1.8836 & 0 & 1.0404 & 0 & \\
\hline 174 & Compositae & $\begin{array}{l}\text { Tanacetum parthenium (L.) } \\
\text { Sch.Bip. }\end{array}$ & $\begin{array}{l}\text { ERL-77, ERL-153, ERL-178, ERL- } \\
\text { 202, SRL-64 }\end{array}$ & Santa María & 3 & $\begin{array}{l}\text { Ornamental }=53, \\
\text { ceremonial }=10\end{array}$ & 0 & & 0 & & 0.0646 & \\
\hline 175 & Compositae & $\begin{array}{l}\text { Taraxacum campylodes } \\
\text { G.E.Haglund }\end{array}$ & ERL-106, SRL-89 & Achicoria & 3 & Ornamental = 18 & 0 & & 0 & & 0.0046 & \\
\hline 176 & Compositae & $\begin{array}{l}\text { Tithonia rotundifolia (Mill.) } \\
\text { S.F.Blake }\end{array}$ & $\begin{array}{l}\text { ERL-1, ERL-42, ERL-76, ERL-157, } \\
\text { ERL-169 }\end{array}$ & Cahual rojo & 3 & $\begin{array}{l}\text { Ornamental }=65, \\
\text { ceremonial }=10\end{array}$ & 0 & & 0.0062 & & 0 & \\
\hline 177 & Compositae & $\begin{array}{l}\text { Tithonia tubaeformis (Jacq.) } \\
\text { Cass. }\end{array}$ & $\begin{array}{l}\text { RLF-177, SRL-126; RLF-57, SRL- } \\
1144\end{array}$ & Cahual & 3 & $\begin{array}{l}\text { Fodder }=80, \\
\text { ornamental }=41\end{array}$ & 0.1501 & 0.1872 & 0.002 & 0.3403 & 0 & \\
\hline 178 & Compositae & $\begin{array}{l}\text { Tridax coronopifolia (Kunth) } \\
\text { Hemsl. }\end{array}$ & SRL-104 & & 1 & & 0 & & 0 & & 0 & -1.0487 \\
\hline 179 & Compositae & Verbesina gracilipes B.L.Rob. & SRL-392 & Chimalacate & 2 & & 0 & -0.6097 & 0 & & 0 & \\
\hline 180 & Compositae & Vernonia karvinskiana DC. & RLF-187, RLF-210 & & 1 & & 0 & & 0 & -2.1063 & 0 & \\
\hline 181 & Compositae & $\begin{array}{l}\text { Viguiera cordata (Hook. \& } \\
\text { Arn.) D'Arcy }\end{array}$ & SRL-95 & $\begin{array}{l}\text { Cahual menudito, } \\
\text { cahual prieto }\end{array}$ & 1 & & 0 & -1.0765 & 0 & & 0 & \\
\hline 182 & Compositae & $\begin{array}{l}\text { Viguiera dentata (Cav.) } \\
\text { Spreng. }\end{array}$ & $\begin{array}{l}\text { RLF-227, SRL-164, SRL-1277, } \\
\text { SRL-1302 }\end{array}$ & Chimalacate & 5 & & 0 & 0.7128 & 0 & & 0 & 0.0591 \\
\hline 183 & Compositae & $\begin{array}{l}\text { Viguiera grammatoglossa } \\
\text { DC. }\end{array}$ & $\begin{array}{l}\text { RLF-233, RLF-298, SRL-347, SRL- } \\
1286\end{array}$ & Cahual prieto & 2 & & 0 & -0.2201 & 0 & & 0 & -0.5074 \\
\hline 184 & Compositae & Viguiera purpusii Brandegee & RLF-248 & Cahual cimarrón & 1 & & 0 & -1.0765 & 0 & & 0 & \\
\hline 185 & Compositae & Zaluzania sp. & RLF-238, SRL-1387 & Cahualito & 1 & & 0 & -1.0765 & 0 & & 0 & \\
\hline 186 & Compositae & Zinnia elegans L. & ERL-156 & Gallito & 2 & Ornamental $=6$ & 0 & & 0 & & 0 & \\
\hline 187 & Compositae & Zinnia peruviana (L.) L. & $\begin{array}{l}\text { RLF-12, RLF-234, SRL-367, SRL- } \\
1173, \text { SRL-1261, SRL-1317 }\end{array}$ & Gallito & 3 & & 0 & 0.3455 & 0 & & 0 & -0.161 \\
\hline 188 & Compositae & & SRL-1421 & & & & 0 & & 0 & & 0 & \\
\hline 191 & Compositae & & SRL-1465 & & & & 0 & & 0 & & 0 & \\
\hline 192 & Compositae & & SRL-1422 & & & & 0 & & 0 & & 0 & \\
\hline 193 & Compositae & & SRL-1527 & & & & 0 & & 0 & & 0 & \\
\hline
\end{tabular}


Table 5 Species, Spanish common names, number of uses, percentage of families that consume it; cognitive prominence values expressed as $\mathrm{S}=$ Sutrop relative prominence index ${ }^{2}$ and biocultural importance expressed as first component value of the principal component analysis by use type (edible, medicinal, firewood, fodder, ceremonial and ornamental; distribution on vegetal types, importance ecological index value (EIVI); specie origin region, ecological status, management practices and management site with respect to species wild populations (Continued)

\begin{tabular}{|c|c|c|c|c|c|c|c|c|c|c|c|}
\hline 194 & Compositae & & SRL-1214 & $\begin{array}{l}\text { Jazmincillo, cahual } \\
\text { blanco }\end{array}$ & 1 & 0 & & 0 & & 0 & \\
\hline 195 & Compositae & & SRL-1236 & & 1 & 0 & -1.0765 & 0 & & 0 & \\
\hline 196 & Compositae & & SRL-1442, SRL-1530 & & & 0 & & 0 & & 0 & \\
\hline 197 & Compositae & & SRL-1372 & & 1 & 0 & & 0 & & 0 & -1.0487 \\
\hline 198 & Compositae & & SRL-1445 & & & 0 & & 0 & & 0 & \\
\hline 199 & Compositae & & SRL-1355 & & & 0 & & 0 & & 0 & \\
\hline 200 & Compositae & & SRL-1381 & Cahual de hembra & & 0 & & 0 & & 0 & \\
\hline 201 & Compositae & & SRL-1407 & & 1 & 0 & -1.0765 & 0 & & 0 & \\
\hline 202 & Compositae & & SRL-1224 & Cahual & 1 & 0 & & 0 & -2.1063 & 0 & \\
\hline 203 & Compositae & & SRL-1205 & & & 0 & & 0 & & 0 & \\
\hline 204 & Compositae & & SRL-1335 & & & 0 & & 0 & & 0 & \\
\hline 205 & Compositae & & SRL-1360 & & & 0 & & 0 & & 0 & \\
\hline 206 & Compositae & & SRL-1337 & & & 0 & & 0 & & 0 & \\
\hline 207 & Compositae & & SRL-1383 & & & 0 & & 0 & & 0 & \\
\hline 208 & Compositae & & SRL-1377 & & & 0 & & 0 & & 0 & \\
\hline 209 & Compositae & & ERL-121, SRL-1275 & Cahual prieto & 1 & 0 & & 0 & & 0 & -0.8133 \\
\hline 210 & Compositae & & SRL-1478 & $\begin{array}{l}\text { Hierba de ángel, } \\
\text { oaxaqueña }\end{array}$ & Medicinal $=15$ & 0 & & 0 & & 0 & 0.0384 \\
\hline 211 & Compositae & & SRL-1339 & $\begin{array}{l}\text { Cempasuchí de molito } \\
\text { de campo }\end{array}$ & 1 & 0 & & 0 & & 0 & -1.0487 \\
\hline 305 & Convolvulaceae & Cuscuta sp. & RLF-264, RLF-315, SRL-447 & & & 0 & & 0 & & 0 & \\
\hline 306 & Convolvulaceae & Cuscuta sp. & SRL-1540, SRL-1545 & & & 0 & & 0 & & 0 & \\
\hline 307 & Convolvulaceae & $\begin{array}{l}\text { Dichondra argentea Humb. } \\
\text { \& Bonpl. ex Wild. }\end{array}$ & RLF-71, SRL-134, SRL-167 & Orejita de ratón & 1 & 0 & & 0 & & 0 & -0.7399 \\
\hline 309 & Convolvulaceae & Ipomoea conzattii Greenm. & SRL-1491, SRL-1510 & Jícama de cerro & 2 & 0 & -0.6097 & 0 & & 0 & \\
\hline 310 & Convolvulaceae & Ipomoea elongata Choisy & $\begin{array}{l}\text { RLF-130, RLF-192, SRL-327, SRL- } \\
1203\end{array}$ & $\begin{array}{l}\text { Manto de la virgen del } \\
\text { campo }\end{array}$ & 1 & 0 & & 0 & -2.1063 & 0 & \\
\hline 311 & Convolvulaceae & $\begin{array}{l}\text { Ipomoea pauciflora } \\
\text { M.Martens \& Galeotti }\end{array}$ & SRL-1366 & & & 0 & & 0 & & 0 & \\
\hline 308 & Convolvulaceae & Ipomoea aff. populina House & SRL-1306 & Jícama & 2 & 0 & -0.6097 & 0 & & 0 & \\
\hline 312 & Convolvulaceae & Ipomoea purpurea (L.) Roth & $\begin{array}{l}\text { ERL-14, RLF-44, RLF-45, SRL-145, } \\
\text { SRL-448 }\end{array}$ & Quiebra platos & 1 & 0 & & 0 & & 0 & -0.7546 \\
\hline
\end{tabular}


Table 5 Species, Spanish common names, number of uses, percentage of families that consume it; cognitive prominence values expressed as $S=$ Sutrop relative prominence index ${ }^{2}$ and biocultural importance expressed as first component value of the principal component analysis by use type (edible, medicinal, firewood, fodder, ceremonial and ornamental; distribution on vegetal types, importance ecological index value (EIVI); specie origin region, ecological status, management practices and management site with respect to species wild populations (Continued)

\begin{tabular}{|c|c|c|c|c|c|c|c|c|c|c|c|c|}
\hline 313 & Convolvulaceae & Ipomoea ternifolia Cav. & SRL-1363 & & 1 & & 0 & -1.0765 & 0 & & 0 & \\
\hline 314 & Convolvulaceae & Ipomoea tricolor Cav & Photo record & Manto de la Virgen & 1 & Ornamental $=12$ & 0 & & 0.0147 & & 0 & \\
\hline 315 & Crassulaceae & $\begin{array}{l}\text { Aeonium arboreum Webb \& } \\
\text { Berthel. }\end{array}$ & Photo record & & 1 & Ornamental $=6$ & 0 & & 0 & & 0 & \\
\hline 316 & Crassulaceae & $\begin{array}{l}\text { Bryophyllum delagoense } \\
\text { (Eckl. \& Zeyh.) Druce }\end{array}$ & ERL-3, SRL-61 & Viborita & 2 & Ornamental $=12$ & 0 & & 0 & & 0 & \\
\hline 317 & Crassulaceae & $\begin{array}{l}\text { Echeveria gigantea Rose \& } \\
\text { Purpus }\end{array}$ & SRL-1313 & $\begin{array}{l}\text { Siempreviva grande, } \\
\text { lengua de vaca, oreja } \\
\text { de toro }\end{array}$ & 2 & Ornamental = 18 & 0 & & 0.0107 & 0.9419 & 0.0025 & 1.7348 \\
\hline 318 & Crassulaceae & $\begin{array}{l}\text { Echeveria nodulosa (Baker) } \\
\text { Otto }\end{array}$ & $\begin{array}{l}\text { SRL-356, SRL-1187, SRL-1255, } \\
\text { SRL-1436 }\end{array}$ & Siempreviva chiquita & 2 & & 0 & & 0.0033 & 0.2914 & 0 & 1.7058 \\
\hline 319 & Crassulaceae & Echeveria pulvinata Rose & Photo record & Siempreviva & 2 & $\begin{array}{l}\text { Ornamental =6, } \\
\text { ceremonial =1 }\end{array}$ & 0 & & 0 & & 0 & \\
\hline 320 & Crassulaceae & Echeveria sp. & Photo record & Siempreviva & 1 & Ornamental $=6$ & 0 & & 0 & -0.0219 & 0 & \\
\hline 321 & Crassulaceae & Echeveria sp. & Photo record & Siempreviva & 1 & & 0 & & 0 & -0.1783 & 0 & \\
\hline 322 & Crassulaceae & $\begin{array}{l}\text { Kalanchoe blossfeldiana } \\
\text { Poelln. }\end{array}$ & ERL-96 & Juanita & 1 & Ornamental $=6$ & 0 & & 0 & & 0 & \\
\hline 323 & Crassulaceae & Kalanchoe sp. & ERL-26, ERL-183, SRL-1552 & Oreja de elefante & 2 & $\begin{array}{l}\text { Ornamental }=41, \\
\text { ceremonial }=14\end{array}$ & 0 & & 0 & & 0 & \\
\hline 324 & Crassulaceae & Sedum allantoides Rose & ERL-67, ERL-192 & Dedito de Dios & 2 & Ornamental $=18$ & 0 & & 0 & & 0 & \\
\hline 325 & Crassulaceae & $\begin{array}{l}\text { Sedum dendroideum Moc. \& } \\
\text { Sessé ex DC. }\end{array}$ & $\begin{array}{l}\text { SRL-77, SRL-195, ERL-97, ERL- } \\
174\end{array}$ & Siempreviva & 3 & $\begin{array}{l}\text { Ornamental }=29, \\
\text { ceremonial }=14\end{array}$ & 0 & & 0.0272 & 2.4485 & 0.0056 & 2.5616 \\
\hline 326 & Crassulaceae & Sedum hemsleyanum Rose & SRL-1311 & & & & 0 & & 0 & & 0 & \\
\hline 327 & Crassulaceae & $\begin{array}{l}\text { Sedum liebmannianum } \\
\text { Hemsl. }\end{array}$ & $\begin{array}{l}\text { ERL-57, ERL-68, SRL-147, SRL- } \\
373, \text { SRL-1174 }\end{array}$ & Siempreviva chiquita & 2 & Ornamental = 6 & 0 & 3.4262 & 0.0037 & 0.9638 & 0 & \\
\hline 328 & Crassulaceae & Sedum stahlii Solms & SRL-1554 & & & & 0 & & 0 & & 0 & \\
\hline 329 & Crassulaceae & Sedum palmeri S.Watson & Photo record & Siempreviva & 1 & Ornamental = 6 & 0 & & 0 & & 0 & \\
\hline 330 & Crassulaceae & Sedum potosinum Rose & Photo record & & 1 & Ornamental $=12$ & 0 & & 0 & & 0 & \\
\hline 331 & Crassulaceae & $\begin{array}{l}\text { Villadia albiflora (Hemsl.) } \\
\text { Rose }\end{array}$ & SRL-1310 & Borreguito & & & 0 & & 0 & & 0 & \\
\hline 332 & Crassulaceae & Villadia guatemalensis Rose & ERL-45, SRL-1312, SRL-1484 & Colita de borrego & 2 & Ornamental $=6$ & 0 & 3.4262 & 0 & 0.8632 & 0 & \\
\hline 336 & Cucurbitaceae & Cucumis melo L. & Photo record & Melón & 1 & & 0 & & 0 & & 0 & \\
\hline 333 & Cucurbitaceae & Cucurbita ficifolia Bouché & Photo record & Chilacayota & 1 & Edible $=100$ & 0 & & 0 & & 0 & \\
\hline 334 & Cucurbitaceae & $\begin{array}{l}\text { Cucurbita pedatifolia } \\
\text { L.H.Bailey }\end{array}$ & ERL-120, RLF-268, SRL-1135 & Calabacita amarga & 3 & & 0 & 0.0916 & 0 & & 0 & -0.3182 \\
\hline
\end{tabular}


Table 5 Species, Spanish common names, number of uses, percentage of families that consume it; cognitive prominence values expressed as $S=$ Sutrop relative prominence index ${ }^{2}$ and biocultural importance expressed as first component value of the principal component analysis by use type (edible, medicinal, firewood, fodder, ceremonial and ornamental; distribution on vegetal types, importance ecological index value (EIVI); specie origin region, ecological status, management practices and management site with respect to species wild populations (Continued)

\begin{tabular}{|c|c|c|c|c|c|c|c|c|c|c|c|c|}
\hline 335 & Cucurbitaceae & Cucurbita pepo L. & SRL-184 & Calabaza & 2 & Edible $=100$ & 0 & & 0 & & 0 & \\
\hline 337 & Cucurbitaceae & $\begin{array}{l}\text { Cyclanthera dissecta (Torr. \& } \\
\text { A.Gray) Arn. }\end{array}$ & SRL-151 & Chayotito & 2 & & 0 & -0.2201 & 0 & & 0 & -0.5074 \\
\hline 338 & Cucurbitaceae & $\begin{array}{l}\text { Schizocarpum filiforme } \\
\text { Schrad. }\end{array}$ & SRL-1260 & Chayotito & 2 & & 0 & -0.2201 & 0 & & 0 & -0.5074 \\
\hline 339 & Cucurbitaceae & $\begin{array}{l}\text { Sechium edule subsp. edule } \\
\text { (Jacq.) Sw. }\end{array}$ & ERL-56, ERL-215 & Chayote & 1 & Edible $=100$ & 0 & & 0 & & 0 & \\
\hline 340 & Cucurbitaceae & Sicyos laciniatus L. & ERL-100, RLF-90, SRL-14 & Chayotillo, pegajosa & 2 & Fodder $=40$ & 0 & -0.0182 & 0 & & 0 & -0.4506 \\
\hline 342 & Cupressaceae & Cupressus sempervirens L. & Photo record & Ciprés & 1 & Ornamental $=24$ & 0 & & 0.0294 & & 0 & \\
\hline 341 & Cupressaceae & $\begin{array}{l}\text { Cupressus lusitanica var. } \\
\text { benthamii (Endl.) Carrière }\end{array}$ & RLF-129, SRL-36 & Nebro fino & 3 & Ornamental = 6 & 0 & & 0 & & 0 & \\
\hline 343 & Cupressaceae & Juniperus flaccida Schltdl. & $\begin{array}{l}\text { ERL-187, RLF-126, RLF-134, SRL- } \\
123, \text { SRL-412, SRL-1119 }\end{array}$ & Nebro & 8 & $\begin{array}{l}\text { Ornamental = 35, } \\
\text { firewood }=100\end{array}$ & 0.0054 & 5.2489 & 0.0147 & 4.8804 & 0 & 3.0378 \\
\hline 344 & Cupressaceae & Taxodium huegelii C.Lawson & SRL-210, SRL-434, SRL-1294 & Sabino & 5 & Ornamental $=6$ & 0 & & 0 & 2.3689 & 0 & \\
\hline 345 & Cupressaceae & Thuja occidentalis L. & ERL-122 & Tuja & 1 & Ornamental $=6$ & 0 & & 0 & & 0 & \\
\hline 347 & Cyperaceae & $\begin{array}{l}\text { Bulbostylis juncoides (Vahl) } \\
\text { Kük. ex Herter }\end{array}$ & SRL-310 & Pasto & 1 & & 0 & -0.4243 & 0 & & 0 & \\
\hline 348 & Cyperaceae & Carex sp. & RLF-133 & Pasto & 2 & & 0 & -0.6097 & 0 & & 0 & -0.8011 \\
\hline 349 & Cyperaceae & $\begin{array}{l}\text { Cyperus aggregatus (Willd.) } \\
\text { Endl. }\end{array}$ & SRL-382 & Pasto & 1 & & 0 & -1.0538 & 0 & & 0 & \\
\hline 351 & Cyperaceae & Cyperus spectabilis Link & RLF-334 & Pasto & & & 0 & & 0 & & 0 & \\
\hline 352 & Cyperaceae & $\begin{array}{l}\text { Eleocharis acicularis (L.) } \\
\text { Roem. \& Schult. }\end{array}$ & RLF-138 & Pasto de arroyo & 1 & & 0 & & 0 & & 0 & -1.0487 \\
\hline 353 & Cyperaceae & $\begin{array}{l}\text { Eleocharis montevidensis } \\
\text { Kunth }\end{array}$ & SRL-197 & Pasto de arroyo & 1 & & 0 & & 0 & & 0 & \\
\hline 346 & Cyperaceae & Fimbristylis mexicana Palla & SRL-304 & Pasto & 1 & & 0 & -0.2720 & 0 & & 0 & \\
\hline 354 & Cyperaceae & Fuirena simplex Vahl & SRL-431 & Pasto & 1 & & 0 & -1.0765 & 0 & & 0 & \\
\hline 350 & Cyperaceae & $\begin{array}{l}\text { Pycreus niger (Ruiz \& Pav.) } \\
\text { Cufod. }\end{array}$ & RLF-144 & Pasto & 1 & & 0 & -1.0765 & 0 & & 0 & \\
\hline 355 & Cyperaceae & Rhynchospora sp. & RLF-145 & Pasto fino & 1 & & 0 & -1.0765 & 0 & & 0 & \\
\hline 356 & Ebenaceae & Diospyros oaxacana Standl. & SRL-1446 & Zapotito & 2 & & 0 & -0.6097 & 0 & & 0 & \\
\hline 357 & Equisetaceae & Equisetum sp. & SRL-422 & & & & 0 & & 0 & & 0 & \\
\hline 358 & Ericaceae & Arbutus xalapensis Kunth & $\begin{array}{l}\text { ERL-172, RLF-124, RLF-279, SRL- } \\
1477\end{array}$ & Madroño, ollita & 4 & $\begin{array}{l}\text { Ceremonial = 14, } \\
\text { firewood }=100\end{array}$ & 0 & & 0 & & 0 & -0.1056 \\
\hline
\end{tabular}


Table 5 Species, Spanish common names, number of uses, percentage of families that consume it; cognitive prominence values expressed as $\mathrm{S}=$ Sutrop relative prominence index ${ }^{2}$ and biocultural importance expressed as first component value of the principal component analysis by use type (edible, medicinal, firewood, fodder, ceremonial and ornamental; distribution on vegetal types, importance ecological index value (EIVI); specie origin region, ecological status, management practices and management site with respect to species wild populations (Continued)

\begin{tabular}{|c|c|c|c|c|c|c|c|c|c|c|c|}
\hline 359 & Ericaceae & $\begin{array}{l}\text { Comarostaphylis polifolia } \\
\text { (Kunth) Zucc. ex Klotzsch }\end{array}$ & $\begin{array}{l}\text { RLF-118, SRL-130, SRL-250, SRL- } \\
1495\end{array}$ & Palo prieto & 3 & Firewood $=100$ & 0 & & 0 & 0 & \\
\hline 360 & Euphorbiaceae & $\begin{array}{l}\text { Acalypha aff. purpurascens } \\
\text { Kunth }\end{array}$ & RLF-189, SRL-256 & & & & 0 & & 0 & 0 & \\
\hline 361 & Euphorbiaceae & Bernardia sp. & SRL-1386 & & & & 0 & & 0 & 0 & \\
\hline 362 & Euphorbiaceae & $\begin{array}{l}\text { Cnidosculus tehuacanensis } \\
\text { Breckon }\end{array}$ & Photo record & Mala mujer & 1 & & 0 & & 0 & 0.0043 & -0.9341 \\
\hline 363 & Euphorbiaceae & Croton sp. & SRL-441 & & & & 0 & & 0 & 0 & \\
\hline 364 & Euphorbiaceae & Croton sp. & SRL-1444 & & & & 0 & & 0 & 0 & \\
\hline 365 & Euphorbiaceae & Euphorbia colletioides Benth. & SRL-1359 & & 1 & & 0 & -1.0765 & 0 & 0 & \\
\hline 366 & Euphorbiaceae & $\begin{array}{l}\text { Euphorbia cyathophora } \\
\text { Murray }\end{array}$ & SRL-1369 & & & & 0 & & 0 & 0 & \\
\hline 367 & Euphorbiaceae & $\begin{array}{l}\text { Euphorbia cymbifera } \\
\text { (Schltdl.) V.W.Steinm. }\end{array}$ & SRL-1500 & & & & 0 & & 0 & 0 & \\
\hline 368 & Euphorbiaceae & Euphorbia cyri V.W.Steinm. & SRL-1128 & Cordobán & 2 & Ornamental $=12$ & 0 & & 0 & 0 & \\
\hline 369 & Euphorbiaceae & Euphorbia dentata Michx. & $\begin{array}{l}\text { RLF-51, SRL-102, SRL-299, SRL- } \\
376\end{array}$ & Lechillo, limil & 1 & & 0.0025 & -0.1758 & 0 & 0 & \\
\hline 370 & Euphorbiaceae & Euphorbia dioeca Kunth & ERL-107, RLF-7, SRL-359 & Celedonia & 1 & & 0 & & 0 & 0 & -0.7546 \\
\hline 371 & Euphorbiaceae & Euphorbia graminea Jacq. & RLF-288, RLF-311, SRL-317 & & 1 & & 0 & & 0 & 0 & \\
\hline 372 & Euphorbiaceae & Euphorbia lactea Haw. & Photo record & & 1 & & 0 & & 0 & 0 & \\
\hline 373 & Euphorbiaceae & $\begin{array}{l}\text { Euphorbia macropus } \\
\text { (Klotzsch \& Garcke) Boiss. }\end{array}$ & SRL-1120 & Hierba de chicle & 2 & & 0 & & 0 & 0 & -0.8599 \\
\hline 374 & Euphorbiaceae & $\begin{array}{l}\text { Euphorbia pulcherrima Willd. } \\
\text { ex Klotzsch }\end{array}$ & Photo record & Noche buena & 2 & $\begin{array}{l}\text { Ornamental }=47, \\
\text { ceremonial }=11\end{array}$ & 0 & & 0.1246 & 0 & \\
\hline 375 & Euphorbiaceae & Euphorbia rossiana Pax & SRL-1450 & & & & 0 & & 0 & 0 & \\
\hline 376 & Euphorbiaceae & Euphorbia sp. & RLF-141 & Mastrancito & & & 0 & & 0 & 0 & \\
\hline 377 & Euphorbiaceae & Euphorbia sp. & RLF-301, SRL-254 & & & & 0 & & 0 & 0 & \\
\hline 378 & Euphorbiaceae & Euphorbia sp. & $\begin{array}{l}\text { RLF-119, RLF-152, RLF-167, SRL- } \\
283\end{array}$ & & & & 0 & & 0 & 0 & \\
\hline 379 & Euphorbiaceae & Jatropha neopauciflora Pax & SRL-1357 & $\begin{array}{l}\text { Sangre de grado, } \\
\text { aceitillo }\end{array}$ & 2 & & 0 & -0.6097 & 0 & 0 & -0.8011 \\
\hline 380 & Euphorbiaceae & Ricinus communis L. & $\begin{array}{l}\text { ERL-1 16, ERL-144, ERL-145, ERL- } \\
243, \text { SRL-23, SRL-1129 }\end{array}$ & Gría & 5 & & 0 & & 0 & 0.0161 & \\
\hline 381 & Euphorbiaceae & $\begin{array}{l}\text { Sebastiania aff. pavoniana } \\
\text { (Müll.Arg.) Müll.Arg. }\end{array}$ & SRL-263 & Hierba de venado & 1 & & 0 & -1.0683 & 0 & 0 & \\
\hline
\end{tabular}

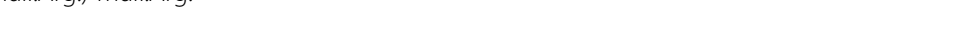


Table 5 Species, Spanish common names, number of uses, percentage of families that consume it; cognitive prominence values expressed as $\mathrm{S}=$ Sutrop relative prominence index ${ }^{2}$ and biocultural importance expressed as first component value of the principal component analysis by use type (edible, medicinal, firewood, fodder, ceremonial and ornamental; distribution on vegetal types, importance ecological index value (EIVI); specie origin region, ecological status, management practices and management site with respect to species wild populations (Continued)

\begin{tabular}{|c|c|c|c|c|c|c|c|c|c|c|c|c|}
\hline 382 & Euphorbiaceae & Tragia nepetifolia Cav. & SRL-318 & & & & 0 & & 0 & & 0 & \\
\hline 383 & Euphorbiaceae & & RLF-252 & & & & 0 & & 0 & & 0 & \\
\hline 384 & Fagaceae & Quercus acutifolia Née & SRL-1226, SRL-1516 & Encino colorado & 7 & Firewood $=100$ & 0.0153 & 3.7957 & 0.0392 & 2.304 & 0.0101 & 2.6129 \\
\hline 385 & Fagaceae & Quercus castanea Née & $\begin{array}{l}\text { RLF-78, SRL-1233, SRL-1408, } \\
\text { SRL-1425, SRL-1431 }\end{array}$ & $\begin{array}{l}\text { Encino prieto, encino } \\
\text { blanco }\end{array}$ & 7 & Firewood $=100$ & 0.0215 & 1.4099 & 0.0392 & 1.4528 & 0 & 0.4908 \\
\hline 386 & Fagaceae & Quercus conspersa Benth. & SRL-1156 & Encino colorado & 7 & Firewood $=100$ & 0.0153 & 0.6176 & 0.0392 & 0.7792 & 0.0101 & 0.1196 \\
\hline 393 & Fagaceae & Quercus x dysophylla Benth. & SRL-1108 & Encino de tesmole & 3 & Firewood $=100$ & 0.0091 & 0.6263 & 0.0392 & 0.5657 & 0 & \\
\hline 387 & Fagaceae & $\begin{array}{l}\text { Quercus glaucoides M. } \\
\text { Martens \& Galeotti }\end{array}$ & $\begin{array}{l}\text { SRL-1 109, SRL-1459, SRL-1486, } \\
\text { SRL-1513 }\end{array}$ & Encino chaparro & 5 & Firewood $=100$ & 0.0161 & 0.3057 & 0.0686 & 1.3213 & 0 & \\
\hline 388 & Fagaceae & Quercus laeta Liebm. & $\begin{array}{l}\text { RLF-68, SRL-143, SRL-253, SRL- } \\
\text { 385, SRL-1230 }\end{array}$ & $\begin{array}{l}\text { Encino prieto, encino } \\
\text { amarillo }\end{array}$ & 6 & $\begin{array}{l}\text { Ornamental =6, } \\
\text { firewood }=100\end{array}$ & 0.0129 & 4.1162 & 0.0392 & 2.6146 & 0 & \\
\hline 389 & Fagaceae & $\begin{array}{l}\text { Quercus liebmannii Oerst. ex } \\
\text { Trel. }\end{array}$ & SRL-1107, SRL-1514 & Encino amarillo & 8 & $\begin{array}{l}\text { Fodder }=5 \\
\text { firewood }=100\end{array}$ & 0.0108 & 6.7493 & 0.0392 & 4.6656 & 0 & \\
\hline 390 & Fagaceae & Quercus obtusata Bonpl. & SRL-1423 & Encino prieto & 6 & Firewood $=100$ & 0.0092 & 0.9366 & 0.0392 & 0.8996 & 0 & \\
\hline 391 & Fagaceae & $\begin{array}{l}\text { Quercus polymorpha Schltdl. } \\
\& \text { Cham. }\end{array}$ & SRL-1503 & Encino prieto & 5 & & 0 & 0.6356 & 0.0392 & 0.6369 & 0 & \\
\hline 392 & Fagaceae & Quercus urbanii Trel & $\begin{array}{l}\text { RLF-161, SRL-252, SRL-475, SRL- } \\
1228\end{array}$ & Encino cucharilla & 6 & Firewood $=100$ & 0.0081 & 1.9079 & 0.0392 & 1.7423 & 0 & \\
\hline 395 & Garryaceae & Garrya ovata Benth. & SRL-330, SRL-469 & Hierba de ardilla & 2 & Firewood $=100$ & 0.0323 & -0.0578 & 0 & & 0 & \\
\hline 396 & Geraniaceae & Geranium sp. & RLF-278, SRL-136 & & & & 0 & & 0 & & 0 & \\
\hline 397 & Geraniaceae & $\begin{array}{l}\text { Pelargonium peltatum (L.) } \\
\text { L'Hér. }\end{array}$ & Photo record & Geranio, malva rosa & 2 & Ornamental $=6$ & 0 & & 0 & & 0 & \\
\hline 398 & Geraniaceae & $\begin{array}{l}\text { Pelargonium zonale (L.) } \\
\text { L'Hér. ex Aiton }\end{array}$ & ERL-84, ERL-200 & Geranio, malva rosa & 2 & $\begin{array}{l}\text { Ornamental }=88, \\
\text { ceremonial }=43\end{array}$ & 0 & & 0.0888 & & 0 & \\
\hline 399 & Geraniaceae & & SRL-81 & & & & 0 & & 0 & & 0 & \\
\hline 400 & Hydrangeaceae & $\begin{array}{l}\text { Hydrangea macrophylla } \\
\text { (Thunb.) Ser. }\end{array}$ & Photo record & Hortensia & 2 & & 0 & & 0 & & 0 & \\
\hline 404 & Hypoxidaceae & Hypoxis sp. & RLF-37, SRL-141 & Pasto & 2 & & 0 & -0.5563 & 0 & & 0 & \\
\hline 405 & Iridaceae & $\begin{array}{l}\text { Gladiolus hortulanus L.H. } \\
\text { Bailey }\end{array}$ & Photo record & Gladiolo & 2 & $\begin{array}{l}\text { Ornamental }=41 \\
\text { ceremonial }=22\end{array}$ & 0 & & 0 & & 0 & \\
\hline 406 & Iridaceae & Iris $\times$ germanica $\mathrm{L}$. & SRL-225 & Lirio corriente & 2 & Ornamental $=29$ & 0 & & 0 & & 0 & \\
\hline 407 & Iridaceae & Neomarica sp. & Photo record & Lirio & 2 & & 0 & & 0 & & 0 & \\
\hline 408 & Iridaceae & $\begin{array}{l}\text { Sisyrinchium tenuifolium } \\
\text { Humb. \& Bonpl. ex Willd. }\end{array}$ & RLF-146, SRL-1548 & $\begin{array}{l}\text { Hierba de camino } \\
\text { corriente }\end{array}$ & 1 & & 0 & -0.9652 & 0 & & 0 & \\
\hline 409 & Iridaceae & Tigridia illecebrosa Cruden & RJS-10 & Flor de gamito & 2 & & 0 & -0.7655 & 0 & -1.4404 & 0 & \\
\hline
\end{tabular}


Table 5 Species, Spanish common names, number of uses, percentage of families that consume it; cognitive prominence values expressed as $S=$ Sutrop relative prominence index ${ }^{2}$ and biocultural importance expressed as first component value of the principal component analysis by use type (edible, medicinal, firewood, fodder, ceremonial and ornamental; distribution on vegetal types, importance ecological index value (EIVI); specie origin region, ecological status, management practices and management site with respect to species wild populations (Continued)

\begin{tabular}{|c|c|c|c|c|c|c|c|c|c|c|c|c|}
\hline 410 & Iridaceae & Tigridia pavonia (L.f.) DC. & RLF-201 & & 1 & & 0 & & 0 & -2.1063 & 0 & \\
\hline 669 & Iteaceae & $\begin{array}{l}\text { Pterostemon rotundifolius } \\
\text { Ramírez }\end{array}$ & RLF-272, RLF-273, SRL-331 & $\begin{array}{l}\text { Encino redondo o } \\
\text { chaparro }\end{array}$ & 1 & Firewood $=100$ & 0 & & 0 & & 0 & \\
\hline 411 & Juglandaceae & Juglans regia L. & ERL-193 & Nuez & 1 & & 0 & & 0 & & 0 & \\
\hline 412 & Krameriaceae & Krameria cytisoides Cav. & $\begin{array}{l}\text { RLF-97, SRL-251, SRL-1265, SRL- } \\
1376\end{array}$ & $\begin{array}{l}\text { Chayotillo de burro, } \\
\text { borreguito }\end{array}$ & 2 & & 0 & -0.5482 & 0 & & 0.004 & -0.6981 \\
\hline 413 & Lamiaceae & $\begin{array}{l}\text { Clinopodium mexicanum } \\
\text { (Benth.) Govaerts }\end{array}$ & $\begin{array}{l}\text { RLF-131, RLF-262, SRL-1190, } \\
\text { SRL-1280, SRL-1403 }\end{array}$ & Chipito & 2 & Medicinal $=5$ & 0 & & 0 & & 0.1359 & 4.2857 \\
\hline 414 & Lamiaceae & Hyptis sp. & RLF-38 & & & & 0 & & 0 & & 0 & \\
\hline 415 & Lamiaceae & Hyptis sp. & SRL-209 & & 1 & & 0 & & 0 & & 0 & -0.8137 \\
\hline 416 & Lamiaceae & Leonotis nepetifolia (L.) R.Br. & SRL-1315 & & 2 & Ornamental $=6$ & 0 & & 0 & & 0 & \\
\hline 417 & Lamiaceae & Marrubium vulgare L. & $\begin{array}{l}\text { ERL-80, RLF-64, SRL-29, SRL- } \\
1146\end{array}$ & Manrrubio & 1 & Medicinal $=10$ & 0 & & 0 & & 0.056 & \\
\hline 418 & Lamiaceae & Mentha × piperita L. & $\begin{array}{l}\text { ERL-19, ERL-61, ERL-95, SRL-70, } \\
\text { SRL-1137 }\end{array}$ & Hierba buena & 3 & Medicinal = 35 & 0 & & 0 & & 0.0296 & \\
\hline 419 & Lamiaceae & Ocimum basilicum L. & ERL-186, ERL-211, SRI-176 & Albhacar & 2 & Ornamental = 18 & 0 & & 0.0294 & & 0.0222 & \\
\hline 420 & Lamiaceae & Origanum majorana L. & $\begin{array}{l}\text { ERL-15, ERL-53, ERL-85, ERL-142, } \\
\text { SRL-73, SRL-206 }\end{array}$ & Orégano & 3 & Medicinal $=5$ & 0 & & 0 & & 0 & \\
\hline 421 & Lamiaceae & $\begin{array}{l}\text { Plectranthus hadiensis } \\
\text { (Forssk.) Schweinf. ex } \\
\text { Sprenger }\end{array}$ & ERL-212 & & 1 & & 0 & & 0 & & 0 & \\
\hline 422 & Lamiaceae & Rosmarinus officinalis $\mathrm{L}$. & Photo record & Romero cimarrón & 2 & & 0 & & 0 & & 0.0093 & \\
\hline 424 & Lamiaceae & $\begin{array}{l}\text { Salvia aspera M.Martens \& } \\
\text { Galeotti }\end{array}$ & SRL-345, SRL-1263 & Oreganillo & 1 & & 0 & -0.9559 & 0 & & 0 & \\
\hline 425 & Lamiaceae & $\begin{array}{l}\text { Salvia candicans M.Martens } \\
\text { \& Galeotti }\end{array}$ & SRL-155, SRL-1456 & & 1 & & 0 & & 0 & & 0 & -1.0487 \\
\hline 423 & Lamiaceae & Salvia circinnata Cav. & RLF-215, SRL-1291 & & 1 & & 0 & & 0 & & 0 & -1.0487 \\
\hline 426 & Lamiaceae & Salvia keerlii Benth. & SRL-155, SRL-1456 & Oreganillo & & & 0 & & 0 & & 0 & \\
\hline 427 & Lamiaceae & Salvia oaxacana Fernald & RLF-232, SRL-1161, SRL-1188 & Mirto cimarrón & 2 & & 0 & -0.6097 & 0 & & 0 & -0.8011 \\
\hline 428 & Lamiaceae & Salvia pannosa Fernald & RLF-181 & & & & 0 & & 0 & & 0 & \\
\hline 429 & Lamiaceae & Salvia purpurea Cav. & $\begin{array}{l}\text { RLF-1, RLF-194, SRL-116, SRL- } \\
\text { 273, SRL-1195, SRL-1202, SRL- } \\
\text { 1397, SRL-1420 }\end{array}$ & Terciopelo & 3 & & 0 & & 0 & & 0 & -0.5649 \\
\hline 430 & Lamiaceae & Salvia sessei Benth. & RLF-33, RLF-195, SRL-1162 & Oaxaqueña & 1 & & 0 & & 0 & & 0 & -1.0487 \\
\hline 431 & Lamiaceae & Salvia thymoides Benth. & RLF-245, SRL-1469 & Oreganillo cenizo & 1 & & 0 & & 0 & & 0 & -1.0487 \\
\hline
\end{tabular}


Table 5 Species, Spanish common names, number of uses, percentage of families that consume it; cognitive prominence values expressed as $\mathrm{S}=$ Sutrop relative prominence index ${ }^{2}$ and biocultural importance expressed as first component value of the principal component analysis by use type (edible, medicinal, firewood, fodder, ceremonial and ornamental; distribution on vegetal types, importance ecological index value (EIVI); specie origin region, ecological status, management practices and management site with respect to species wild populations (Continued)

\begin{tabular}{|c|c|c|c|c|c|c|c|c|c|c|c|}
\hline 432 & Lamiaceae & Salvia tiliifolia Vahl & ERL-28-ERL-112, RLF-162, SRL-3 & Chía & 2 & & 0 & & 0 & 0 & -0.5632 \\
\hline 433 & Lamiaceae & Salvia villosa Fernald & SRL-285 & & & & 0 & & 0 & 0 & \\
\hline 434 & Lamiaceae & Salvia sp. & Photo record & Mirto & 1 & & 0 & & 0 & 0.0035 & -0.7569 \\
\hline 435 & Lamiaceae & Salvia sp. & RLF-20 & & & & 0 & & 0 & 0 & \\
\hline 436 & Lamiaceae & Salvia sp. & RLF-150 & & & & 0 & & 0 & 0 & \\
\hline 437 & Lamiaceae & Salvia sp. & SRL-140 & Marrubio macho & 1 & & 0 & & 0 & 0 & -1.0487 \\
\hline 438 & Lamiaceae & & SRL-1304 & & 1 & & 0 & -1.0765 & 0 & 0 & \\
\hline 439 & Lamiaceae & & SRL-1448 & & & & 0 & & 0 & 0 & \\
\hline 440 & Lauraceae & Litsea glaucescens Kunth & SRL-1157, SRL-1515 & Laurel & 3 & Ceremonial $=2$ & 0 & & 0 & 0 & \\
\hline 441 & Lauraceae & Persea americana Mill. & $\begin{array}{l}\text { ERL-52, ERL-65, RLF-106, SRL- } \\
432\end{array}$ & Aguacate & 2 & Edible $=100$ & 0 & & 0 & 0.0013 & \\
\hline 442 & Leguminosae & Acacia cochliacantha Willd. & SRL-1374 & Guaje de espino & 1 & & 0 & -1.0765 & 0 & 0 & \\
\hline 443 & Leguminosae & Acacia farnesiana (L.) Willd. & Photo record & Espino & 2 & & 0.0086 & -0.2900 & 0 & 0 & \\
\hline 444 & Leguminosae & $\begin{array}{l}\text { Acacia pennatula (Schltdl. \& } \\
\text { Cham.) Benth. }\end{array}$ & SRL-1471 & Espino & 2 & & 0.0076 & 0.0810 & 0 & 0 & \\
\hline 445 & Leguminosae & $\begin{array}{l}\text { Acacia schaffneri (S.Watson) } \\
\text { F.J.Herm. }\end{array}$ & SRL-183,SRL-460 & Espino & 3 & & 0.0068 & 0.0056 & 0 & 0 & \\
\hline 446 & Leguminosae & $\begin{array}{l}\text { Acaciella tequilana } \\
\text { (S.Watson) Britton \& Rose }\end{array}$ & RLF-53 & Barba de chivo & 1 & & 0 & -1.0765 & 0 & 0 & \\
\hline 447 & Leguminosae & Bauhinia sp. & SRL-160, SRL-1443 & & & & 0 & & 0 & 0 & \\
\hline 448 & Leguminosae & Calliandra sp. & SRL-276 & Guaje de gamito & 2 & Edible $=6$ & 0 & -0.63 & 0 & 0 & \\
\hline 449 & Leguminosae & Calliandra sp. & Photo record & Crin de caballo & & & 0 & & 0 & 0 & \\
\hline 450 & Leguminosae & $\begin{array}{l}\text { Calliandropsis nervosus } \\
\text { (Britton \& Rose) H.M.Hern. \& } \\
\text { P. }\end{array}$ & SRL-1511 & & & & 0 & & 0 & 0 & \\
\hline 451 & Leguminosae & Canavalia villosa Benth. & RLF-226, SRL-1439 & & 1 & & 0 & -1.0765 & 0 & 0 & \\
\hline 452 & Leguminosae & $\begin{array}{l}\text { Cologania broussonetii (Balb.) } \\
\text { DC. }\end{array}$ & SRL-106 & & 1 & & 0 & -1.0765 & 0 & 0 & \\
\hline 453 & Leguminosae & Cologania sp. & RLF-153 & Hierba de venado & 1 & & 0 & -1.0765 & 0 & 0 & \\
\hline 454 & Leguminosae & Cologania sp. & SRL-324 & Lentejilla corriente & 1 & & 0 & -0.7835 & 0 & 0 & \\
\hline 455 & Leguminosae & Crotalaria pumila Ortega & SRL-103, SRL-364 & & 2 & & 0 & -0.6097 & 0 & 0 & -0.8011 \\
\hline 456 & Leguminosae & Crotalaria sp. & SRL-13 & & & & 0 & & 0 & 0 & \\
\hline 457 & Leguminosae & Dalea bicolor Willd. & SRL-1461 & & & & 0 & & 0 & 0 & \\
\hline
\end{tabular}


Table 5 Species, Spanish common names, number of uses, percentage of families that consume it; cognitive prominence values expressed as $S=$ Sutrop relative prominence index ${ }^{2}$ and biocultural importance expressed as first component value of the principal component analysis by use type (edible, medicinal, firewood, fodder, ceremonial and ornamental; distribution on vegetal types, importance ecological index value (EIVI); specie origin region, ecological status, management practices and management site with respect to species wild populations (Continued)

\begin{tabular}{|c|c|c|c|}
\hline 458 & Leguminosae & $\begin{array}{l}\text { Dalea carthagenensis (Jacq.) } \\
\text { J.F.Macbr. }\end{array}$ & $\begin{array}{l}\text { RLF-115, RLF-168, RLF-222, SRL- } \\
\text { 154, SRL-417, SRL-1185, SRL- } \\
1299\end{array}$ \\
\hline 459 & Leguminosae & Dalea hegewischiana Steud. & SRL-1283 \\
\hline 460 & Leguminosae & $\begin{array}{l}\text { Dalea tomentosa (Cav.) } \\
\text { Willd. }\end{array}$ & RLF-214, SRL-214 \\
\hline 461 & Leguminosae & Dalea sp. & RLF-328 \\
\hline 462 & Leguminosae & Dalea sp. & SRL-348 \\
\hline 463 & Leguminosae & Dalea sp. & SRL-111, SRL-168 \\
\hline 465 & Leguminosae & $\begin{array}{l}\text { Desmanthus virgatus (L.) } \\
\text { Willd. }\end{array}$ & SRL-368 \\
\hline 464 & Leguminosae & Desmanthus sp. & RLF-225 \\
\hline 466 & Leguminosae & Desmodium axillare (Sw.) DC. & $\begin{array}{l}\text { RLF-74, SRL-101, SRL-286, SRL- } \\
425\end{array}$ \\
\hline 467 & Leguminosae & $\begin{array}{l}\text { Desmodium orbiculare } \\
\text { Schltdl. }\end{array}$ & RLF-216, SRL-1269 \\
\hline 468 & Leguminosae & $\begin{array}{l}\text { Desmodium subsessile } \\
\text { Schltdl. }\end{array}$ & RLF-114 \\
\hline 469 & Leguminosae & Erythrina americana Mill. & ERL-175, SRL-181, SRL-458 \\
\hline 470 & Leguminosae & $\begin{array}{l}\text { Eysenhardtia polystachya } \\
\text { (Ortega) Sarg. }\end{array}$ & RLF-253, SRL-346, SRL-476 \\
\hline 472 & Leguminosae & Harpalyce formosa DC. & RLF-176, RLF-286, SRL-343 \\
\hline 473 & Leguminosae & Havardia sp. & RLF-325 \\
\hline 471 & Leguminosae & $\begin{array}{l}\text { Hybosema ehrenbergii } \\
\text { (Schltdl.) Harms }\end{array}$ & RLF-123, SRL-259 \\
\hline 474 & Leguminosae & Lens culinaris Medik. & Photo record \\
\hline 475 & Leguminosae & $\begin{array}{l}\text { Leucaena esculenta (DC.) } \\
\text { Benth. }\end{array}$ & $\begin{array}{l}\text { ERL-31, ERL-87, ERL-110, RLF- } \\
\text { 107, RLF-174, SRL-1167, SRL- } \\
1216, \text { SRL-1251, SRL-1343 }\end{array}$ \\
\hline 476 & Leguminosae & $\begin{array}{l}\text { Leucaena leucocephala } \\
\text { (Lam.) de Wit }\end{array}$ & ERL-88, ERL-209 \\
\hline 478 & Leguminosae & Leucaena sp. & SRL-1158 \\
\hline 477 & Leguminosae & $\begin{array}{l}\text { Lupinus leptophyllus Cham. \& } \\
\text { Schltdl. }\end{array}$ & SRL-1410 \\
\hline 479 & Leguminosae & $\begin{array}{l}\text { Macroptilium atropurpureum } \\
\text { (DC.) Urb. }\end{array}$ & SRL-426 \\
\hline
\end{tabular}

Hierba de Obo
Guajito de gabito
Tepeguaje cimarrón
Lentejilla corriente
Papaloquelite de chivo
Gierba de pipi
Coatillo
Guaje de caballo
Guajia caballo, guaje de
Guajillo de chivo
Lenteja
ruaje corde gañada,

\begin{tabular}{|c|c|c|c|c|c|}
\hline & 0 & -0.2201 & 0 & 0.0096 & -0.5388 \\
\hline & 0 & & 0 & 0 & \\
\hline & 0 & -0.5455 & 0 & 0 & -0.7614 \\
\hline & 0 & -1.0765 & 0 & 0 & \\
\hline & 0 & & 0 & 0 & \\
\hline & 0 & & 0 & 0 & \\
\hline & 0 & & 0 & 0 & \\
\hline & 0 & -0.6097 & 0 & 0 & \\
\hline & 0 & -0.3076 & 0 & 0 & \\
\hline & 0.0036 & -1.0538 & 0 & 0 & \\
\hline & 0 & -0.9207 & 0 & 0 & \\
\hline & 0.0023 & & 0 & 0.0025 & \\
\hline Ornamental = 6, & 0.0194 & 0.5698 & -0.1759 & 0 & \\
\hline & 0 & -1.06 & 0 & 0 & \\
\hline & 0 & & 0 & 0 & \\
\hline & 0 & -0.8214 & 0 & 0 & \\
\hline Edible $=100$ & 0 & & 0 & 0 & \\
\hline $\begin{array}{l}\text { Ornamental =94, } \\
\text { edible }=100 \\
\text { firewood }=100\end{array}$ & 0.0161 & & 0 & 0 & \\
\hline Edible $=47$ & 0 & & 0 & 0 & \\
\hline Edible $=6$ & 0 & & 0 & 0 & \\
\hline & 0 & & 0 & 0 & \\
\hline & 0 & -1.0539 & 0 & 0 & \\
\hline
\end{tabular}


Table 5 Species, Spanish common names, number of uses, percentage of families that consume it; cognitive prominence values expressed as $\mathrm{S}=$ Sutrop relative prominence index ${ }^{2}$ and biocultural importance expressed as first component value of the principal component analysis by use type (edible, medicinal, firewood, fodder, ceremonial and ornamental; distribution on vegetal types, importance ecological index value (EIVI); specie origin region, ecological status, management practices and management site with respect to species wild populations (Continued)

\begin{tabular}{|c|c|c|c|c|c|c|c|c|c|c|c|c|}
\hline 480 & Leguminosae & $\begin{array}{l}\text { Macroptilium gibossifolium } \\
\text { (Ortega) A.Delgado }\end{array}$ & RLF-63, SRL-108 & & 2 & & 0 & -0.7428 & 0 & & 0 & \\
\hline 481 & Leguminosae & Medicago lupulina L. & SRL-192 & & 1 & & 0 & & 0 & & 0 & \\
\hline 482 & Leguminosae & Medicago polymorpha L. & RLF-69, SRL-15, SRL-1328 & & 1 & & 0 & & 0 & & 0 & \\
\hline 483 & Leguminosae & Melilotus indicus (L.) All. & SRL-88, SRL-120 & & 1 & & 0 & & 0 & & 0 & \\
\hline 484 & Leguminosae & Mimosa lacerata Rose & RLF-283 & Espino & 1 & & 0 & & 0 & & 0 & \\
\hline 485 & Leguminosae & Mimosa sp. & RLF-85 & Garabato, espino & 1 & & 0 & & 0 & & 0 & \\
\hline 486 & Leguminosae & Nissolia sp. & RLF-163 & & & & 0 & & 0 & & 0 & \\
\hline 487 & Leguminosae & $\begin{array}{l}\text { Parkinsonia praecox (Ruiz \& } \\
\text { Pav.) Hawkins }\end{array}$ & SRL-1396 & Palo verde & & & 0 & & 0 & & 0 & \\
\hline 488 & Leguminosae & Phaseolus coccineus L. & ERL-7, ERL-161 & Frijol ayocote & 2 & Edible $=12$ & 0 & & 0 & & 0 & \\
\hline 489 & Leguminosae & Phaseolus vulgaris L. & $\begin{array}{l}\text { ERL-8, ERL-47, ERL-48, ERL-49, } \\
\text { ERL-139, ERL-160, SRL-9 }\end{array}$ & $\begin{array}{l}\text { Frijol de tierra, frijol de } \\
\text { milpa, bayo, amarillo, } \\
\text { negro, enredador }\end{array}$ & 2 & Edible $=100$ & 0.0352 & & 0 & & 0 & \\
\hline 490 & Leguminosae & Phaseolus sp. & SRL-144 & & 1 & & 0 & -1.0765 & 0 & & 0 & \\
\hline 491 & Leguminosae & Phaseolus sp. & RLF-169 & & & & 0 & & 0 & & 0 & \\
\hline 492 & Leguminosae & Phaseolus sp. & SRL-1206 & Ejote de venado & 2 & & 0 & -0.6097 & 0 & & 0 & \\
\hline 493 & Leguminosae & Phaseolus sp. & SRL-1231 & & 1 & & 0 & -1.0765 & 0 & & 0 & \\
\hline 494 & Leguminosae & $\begin{array}{l}\text { Piscidia grandifolia } \\
\text { (Donn.Sm.) I.M.Johnst. }\end{array}$ & SRL-1210 & & 2 & & 0 & & 0 & & 0 & -0.8599 \\
\hline 495 & Leguminosae & Pisum sativum $\mathrm{L}$. & Photo record & Alberjón & 1 & & 0 & & 0 & & 0 & \\
\hline 496 & Leguminosae & $\begin{array}{l}\text { Prosopis laevigata (Willd.) } \\
\text { M.C.Johnst. }\end{array}$ & SRL-1388 & Mezquite & 5 & & 0 & 0.4025 & 0 & & 0.0035 & -0.1182 \\
\hline 497 & Leguminosae & Rhynchosia pringlei Rose & RLF-247, SRL-1440 & Hierba de venado & 1 & & 0 & -1.0765 & 0 & & 0 & \\
\hline 498 & Leguminosae & Rhynchosia senna Hook. & SRL-284, SRL-366 & & 1 & & 0 & -1.0598 & 0 & & 0 & \\
\hline 499 & Leguminosae & $\begin{array}{l}\text { Senna guatemalensis } \\
\text { (Donn.Sm.) H.S.Irwin \& } \\
\text { Barneby }\end{array}$ & RLF-246, RLF-295 & & 3 & Ceremonial = 1 & 0 & -0.2593 & 0 & & 0 & -0.588 \\
\hline 500 & Leguminosae & $\begin{array}{l}\text { Senna holwayana (Rose) } \\
\text { H.S.Irwin \& Barneby }\end{array}$ & $\begin{array}{l}\text { ERL-223, RLF-75, RLF-230, SRL- } \\
1437\end{array}$ & Mostaza corriente & 2 & Ornamental = 6 & 0 & -0.4532 & 0 & -1.0925 & 0 & \\
\hline 501 & Leguminosae & $\begin{array}{l}\text { Teramnus labialis (L.f.) } \\
\text { Spreng. }\end{array}$ & SRL-396 & & & & 0 & & 0 & & 0 & \\
\hline 502 & Leguminosae & Trifolium sp. & SRL-375 & & 2 & & 0 & & 0 & & 0 & \\
\hline 503 & Leguminosae & Vicia faba L. & Photo record & Haba & 1 & & 0 & & 0 & & 0 & \\
\hline 504 & Leguminosae & Zornia reticulata Sm. & SRL-300 & & 2 & & 0 & -0.5973 & 0 & & 0 & -0.7935 \\
\hline
\end{tabular}


Table 5 Species, Spanish common names, number of uses, percentage of families that consume it; cognitive prominence values expressed as $\mathrm{S}=$ Sutrop relative prominence index ${ }^{2}$ and biocultural importance expressed as first component value of the principal component analysis by use type (edible, medicinal, firewood, fodder, ceremonial and ornamental; distribution on vegetal types, importance ecological index value (EIVI); specie origin region, ecological status, management practices and management site with respect to species wild populations (Continued)

\begin{tabular}{|c|c|c|c|c|c|c|c|c|c|c|c|c|}
\hline 505 & Leguminosae & & RLF-327, SRL-1227 & Timbre & 5 & & 0.0029 & 0.3201 & 0 & & 0 & \\
\hline 506 & Leguminosae & & SRL-1212 & Tepeguaje & 3 & & 0 & -0.4545 & 0 & & 0 & -0.7298 \\
\hline 507 & Leguminosae & & SRL-1556 & & & & 0 & & 0 & & 0 & \\
\hline 508 & Leguminosae & & SRL-1538 & & & & 0 & & 0 & & 0 & \\
\hline 509 & Leguminosae & & SRL-1113 & $\begin{array}{l}\text { Guaje que come el } \\
\text { venado }\end{array}$ & & & 0 & & 0 & & 0 & \\
\hline 510 & Leguminosae & & RJS-7 & & 1 & & 0 & -1.0765 & 0 & & 0 & \\
\hline 511 & Leguminosae & & SRL-1166 & Timbre & 1 & & 0 & & 0 & -0.0825 & 0 & \\
\hline 512 & Leguminosae & & SRL-1350 & & & & 0 & & 0 & & 0 & \\
\hline 513 & Leguminosae & & SRL-1370 & Guaje de gamito & & & 0 & & 0 & & 0 & \\
\hline 514 & Leguminosae & & SRL-1371 & Espino & & & 0 & & 0 & & 0 & \\
\hline 515 & Leguminosae & & SRL-1498 & & & & 0 & & 0 & & 0 & \\
\hline 516 & Leguminosae & & SRL-1217 & & 2 & & 0 & -0.6097 & 0 & & 0 & -0.8011 \\
\hline 517 & Lentibulariaceae & Pinguicula moranensis Kunth & $\begin{array}{l}\text { RLF-148, SRL-436, SRL-1553, } \\
\text { SRL, } 1496\end{array}$ & Siempreviva & & & 0 & & 0 & & 0 & \\
\hline 518 & Linaceae & Linum scabrellum Planch. & SRL-1462 & & & & 0 & & 0 & & 0 & \\
\hline 519 & Linaceae & Linum sp. & RLF-175 & & 2 & & 0 & -0.2201 & 0 & & 0 & -0.5074 \\
\hline 520 & Loasaceae & Mentzelia hispida Willd. & RLF-54, RLF-94, SRL-428 & Pegajosa & 1 & & 0 & & 0 & & 0 & -0.755 \\
\hline 521 & Loranthaceae & $\begin{array}{l}\text { Psittacanthus calyculatus } \\
\text { (DC.) G.Don }\end{array}$ & SRL-1502 & Injerto & 1 & & 0 & -0.7648 & 0 & & 0 & \\
\hline 522 & Lythraceae & Cuphea sp. & $\begin{array}{l}\text { RLF-100, RLF-143, RLF-172, SRL- } \\
\text { 20, SRL-350, SRL-1178 }\end{array}$ & & 3 & & 0 & 0.0939 & 0 & & 0 & -0.3167 \\
\hline 523 & Lythraceae & Cuphea sp. & SRL-25 & & 1 & & 0 & & 0 & & 0 & \\
\hline 524 & Lythraceae & Cuphea sp. & SRL-105, SRL-296 & & 1 & & 0 & & 0 & & 0 & \\
\hline 670 & Lythraceae & Punica granatum $\mathrm{L}$. & $\begin{array}{l}\text { ERL-38, ERL-39, ERL-70, ERL-71, } \\
\text { ERL-104, ERL-206, SRL-43 }\end{array}$ & Granada & 5 & $\begin{array}{l}\text { Ornamental = } 71 \\
\text { edible }=10\end{array}$ & 0 & & 0.0147 & & 0 & \\
\hline 525 & Malpighiaceae & Bunchosia sp. & SRL-451 & Huevo de gato & 2 & & 0 & & 0 & & 0 & \\
\hline 526 & Malpighiaceae & Bunchosia sp. & SRL-1351 & & & & 0 & & 0 & & 0 & \\
\hline 527 & Malpighiaceae & $\begin{array}{l}\text { Echinopterys eglandulosa } \\
\text { (A.Juss.) Small }\end{array}$ & SRL-1384 & & & & 0 & & 0 & & 0 & \\
\hline 528 & Malpighiaceae & Galphimia multicaulis A.Juss. & RLF-65, RLF-293, SRL-1177 & Flor de chivo & 2 & & 0 & -0.5325 & 0 & & 0 & \\
\hline 529 & Malpighiaceae & $\begin{array}{l}\text { Gaudichaudia galeottiana } \\
\text { (Nied.) Chodat }\end{array}$ & RLF-241 & & 1 & & 0 & & 0 & & 0 & -1.0487 \\
\hline
\end{tabular}


Table 5 Species, Spanish common names, number of uses, percentage of families that consume it; cognitive prominence values expressed as $S=$ Sutrop relative prominence index ${ }^{2}$ and biocultural importance expressed as first component value of the principal component analysis by use type (edible, medicinal, firewood, fodder, ceremonial and ornamental; distribution on vegetal types, importance ecological index value (EIVI); specie origin region, ecological status, management practices and management site with respect to species wild populations (Continued)

\begin{tabular}{|c|c|c|c|c|c|c|c|c|c|c|c|c|}
\hline 530 & Malpighiaceae & $\begin{array}{l}\text { Heteropterys brachiata (L.) } \\
\text { DC. }\end{array}$ & SRL-1342 & & & & 0 & & 0 & & 0 & \\
\hline 531 & Malpighiaceae & Malpighia galeottiana A.Juss. & SRL-362, SRL-471, SRL-1272 & Nanche & 4 & Edible $=10$ & 0.0018 & 0.3567 & 0 & & 0 & \\
\hline 532 & Malvaceae & Alcea rosea $\mathrm{L}$. & $\begin{array}{l}\text { ERL-140, ERL-201, ERL-227, SRL- } \\
62, \text { SRL-187 }\end{array}$ & Flor de San José & 2 & Ornamental = 29 & 0 & & 0.0042 & & 0 & \\
\hline 533 & Malvaceae & Anoda cristata (L.) Schltdl. & $\begin{array}{l}\text { RLF-67, RLF-277, SRL-6, SRL-446, } \\
\text { SRL-1125 }\end{array}$ & $\begin{array}{l}\text { Quelite de malva, } \\
\text { violeta }\end{array}$ & 4 & $\begin{array}{l}\text { Fodder }=40, \\
\text { ornamental }=6 \text {, } \\
\text { edible }=5\end{array}$ & 0 & 0.5126 & 0 & -0.4235 & 0 & -0.1293 \\
\hline 534 & Malvaceae & Gossypium hirsutum L. & Photo record & Algodón & 1 & & 0 & & 0 & & 0 & \\
\hline 755 & Malvaceae & $\begin{array}{l}\text { Hermannia inflata Link \& } \\
\text { Otto }\end{array}$ & SRL-1301 & & & & 0 & & 0 & & 0 & \\
\hline 535 & Malvaceae & Hibiscus rosa-sinensis $\mathrm{L}$. & ERL-207 & Tulipán & 2 & Ornamental $=6$ & 0 & & 0.0042 & & 0 & \\
\hline 536 & Malvaceae & Hibiscus sp. & SRL-1474 & & 1 & & 0 & & 0 & & 0 & \\
\hline 537 & Malvaceae & Malva parviflora $\mathrm{L}$. & $\begin{array}{l}\text { ERL-30, ERL-90, SRL-205, SRL- } \\
1124, \text { SRL-1143 }\end{array}$ & Malva & 3 & $\begin{array}{l}\text { Fodder }=5 \\
\text { ornamental }=47 \\
\text { medicinal }=50\end{array}$ & 0.0194 & & 0 & & 0.0324 & \\
\hline 538 & Malvaceae & Malva sylvestris $\mathrm{L}$. & ERL-111, ERL-210 & $\begin{array}{l}\text { Malva rosa, malva de } \\
\text { castilla }\end{array}$ & 2 & Ornamental = 12 & 0 & & 0 & & 0 & \\
\hline 539 & Malvaceae & Sida sp. & SRL-21 & & 1 & & 0 & -1.0765 & 0 & & 0 & \\
\hline 540 & Martyniaceae & $\begin{array}{l}\text { Proboscidea louisianica (Mill.) } \\
\text { Thell. }\end{array}$ & SRL-1318 & Cuerno de toro & 1 & & 0 & & 0 & & 0 & \\
\hline 541 & Meliaceae & Cedrela sp. & ERL-60 & & 1 & Ornamental = 18 & 0 & & 0 & & 0 & \\
\hline 542 & Meliaceae & Melia azedarach L. & ERL-2, SRL-53 & Clavo, paraíso & 2 & Ornamental $=6$ & 0 & & 0 & & 0 & \\
\hline 543 & Meteoriaceae & $\begin{array}{l}\text { Meteorium deppei (Hornsch. } \\
\text { ex Müll. Hal.) Mitt. }\end{array}$ & SRL-1432 & Musgo & 2 & Ceremonial $=2$ & 0 & & 0.0165 & 0.6329 & 0 & \\
\hline 544 & Moraceae & Ficus benjamina $\mathrm{L}$. & SRL-1170 & Laurel de la India & 2 & & 0 & & 0.0294 & & 0 & \\
\hline 545 & Moraceae & Ficus carica $\mathrm{L}$. & ERL-125 & Higo & 1 & Edible $=15$ & 0 & & 0 & & 0 & \\
\hline 546 & Moraceae & $\begin{array}{l}\text { Ficus crocata (Miq.) Mart. ex } \\
\text { Miq. }\end{array}$ & SRL-76, SRL-1171 & Amate & 3 & Ornamental = 6 & 0 & & 0.0049 & -0.6478 & 0 & \\
\hline 547 & Moraceae & Ficus microcarpa L. f. & ERL-115 & Laurel & 2 & Ornamental = 18 & 0 & & 0.0294 & & 0 & \\
\hline 548 & Moraceae & Ficus pertusa L.f. & SRL-433 & & & & 0 & & 0 & & 0 & \\
\hline 549 & Moraceae & Morus celtidifolia Kunth & $\begin{array}{l}\text { ERL-55, ERL-78, ERL-55, ERL-78, } \\
\text { ERL-124, ERL-128, ERL-129, ERL- } \\
\text { 214, ERL-220, ERL-221, RLF-92, } \\
\text { SRL-55, SRL-1517 }\end{array}$ & Moral, morera & 8 & $\begin{array}{l}\text { Ornamental = 88, } \\
\text { firewood }=100\end{array}$ & 0.0116 & 1.9551 & 0.0118 & 3.3295 & 0 & \\
\hline
\end{tabular}


Table 5 Species, Spanish common names, number of uses, percentage of families that consume it; cognitive prominence values expressed as $\mathrm{S}=$ Sutrop relative prominence index ${ }^{2}$ and biocultural importance expressed as first component value of the principal component analysis by use type (edible, medicinal, firewood, fodder, ceremonial and ornamental; distribution on vegetal types, importance ecological index value (EIVI); specie origin region, ecological status, management practices and management site with respect to species wild populations (Continued)

\begin{tabular}{|c|c|c|c|c|c|c|c|c|c|c|c|c|}
\hline 550 & Musaceae & Musa $\times$ paradisiaca $\mathrm{L}$. & Photo record & Plátano & 2 & $\begin{array}{l}\text { Ornamental }=12, \\
\text { edible }=100\end{array}$ & 0 & & 0.0074 & & 0 & \\
\hline 551 & Myrtaceae & $\begin{array}{l}\text { Eucalyptus camaldulensis } \\
\text { Dehnh. }\end{array}$ & SRL-203 & Eucalipto & 2 & & 0 & & 0 & & 0.0019 & \\
\hline 552 & Myrtaceae & Psidium guajava $\mathrm{L}$. & SRL-1528 & Guayaba & 1 & & 0 & & 0 & & 0 & \\
\hline 556 & Nyctaginaceae & Boerhavia anisophylla Torr. & $\begin{array}{l}\text { SRL-162, SRL-193, SRL-370, SRL- } \\
\text { 1184, SRL-1303 }\end{array}$ & & 1 & & 0 & -0.5246 & 0 & & 0 & \\
\hline 557 & Nyctaginaceae & $\begin{array}{l}\text { Bougainvillea spectabilis } \\
\text { Willd. }\end{array}$ & SRL-33, SRL-191 & Bugambilia & 3 & Ornamental $=18$ & 0 & & 0.0529 & & 0 & \\
\hline 558 & Nyctaginaceae & Mirabilis jalapa L. & $\begin{array}{l}\text { ERL-29, ERL-99, SRL-11, SRL-421, } \\
\text { SRL-1145 }\end{array}$ & Hierba cuchi, maravilla & 3 & $\begin{array}{l}\text { Fodder }=50, \\
\text { ornamental }=29\end{array}$ & 0 & 0.2319 & 0 & -0.0608 & 0 & -0.3165 \\
\hline 559 & Oleaceae & $\begin{array}{l}\text { Forestiera rotundifolia } \\
\text { (Brandegee) Standl. }\end{array}$ & RLF-306, SRL-1259 & Tlasisle & 3 & & 0.0025 & 0.0567 & 0 & & 0 & \\
\hline 560 & Oleaceae & Fraxinus purpusii Brandegee & SRL-341, SRL-1463, SRL-1512 & Zapotillo, fresno & 3 & Firewood $=100$ & 0.0076 & -0.307 & 0 & & 0 & \\
\hline 561 & Oleaceae & $\begin{array}{l}\text { Fraxinus uhdei (Wenz.) } \\
\text { Lingelsh. }\end{array}$ & SRL-1409 & Fresno & 1 & & 0 & & 0 & & 0 & \\
\hline 562 & Oleaceae & Ligustrum japonicum Thunb. & $\begin{array}{l}\text { ERL-105, ERL-238, SRL-59, SRL- } \\
453\end{array}$ & Trueno & 4 & $\begin{array}{l}\text { Ornamental }=18, \\
\text { ceremonial }=22\end{array}$ & 0 & & 0.0235 & & 0 & \\
\hline 563 & Onagraceae & Fuchsia sp. & SRL-386, SRL-393 & & & & 0 & & 0 & & 0 & \\
\hline 564 & Onagraceae & $\begin{array}{l}\text { Gaura coccinea Nutt. ex } \\
\text { Pursh }\end{array}$ & SRI-17, SRL-411 & Gradiolita & 2 & & 0 & -0.2194 & 0 & & 0 & -0.507 \\
\hline 565 & Onagraceae & Lopezia racemosa Cav. & $\begin{array}{l}\text { ERL-114, SRL-1, SRL-94, SRL- } \\
1323\end{array}$ & & 1 & & 0 & & 0 & & 0 & \\
\hline 566 & Onagraceae & $\begin{array}{l}\text { Oenothera pubescens Willd. } \\
\text { ex Spreng. }\end{array}$ & $\begin{array}{l}\text { RLF-76, RLF-113, SRL-22, SRL-40, } \\
\text { SRL-150, SRL-213 }\end{array}$ & Campanita grande & 2 & Ornamental $=12$ & 0 & & 0 & -0.8404 & 0 & -0.5653 \\
\hline 567 & Onagraceae & $\begin{array}{l}\text { Oenothera rosea L'Her. ex } \\
\text { Aiton }\end{array}$ & SRL-1127, SRL-1322 & Sanguinaria & 2 & Ornamental $=12$ & 0 & & 0 & -0.8404 & 0 & -0.5653 \\
\hline 568 & Orchidaceae & $\begin{array}{l}\text { Barkeria lindleyana subsp. } \\
\text { vanneriana (Rchb.f.) Thien }\end{array}$ & SRL-1509 & Monjita de peña & 2 & Ceremonial $=8$ & 0 & & 0 & 0.1802 & 0 & \\
\hline 569 & Orchidaceae & Corallorhiza sp. & RLF-207 & Flor de jarrita & & & 0 & & 0 & & 0 & \\
\hline 571 & Orchidaceae & $\begin{array}{l}\text { Cyrtopodium macrobulbon } \\
\text { (Lex.) G.A.Romero \& } \\
\text { Carnevali }\end{array}$ & Photo record & Jarrito & 2 & & 0 & -0.1422 & 0 & -1.0573 & 0 & \\
\hline 572 & Orchidaceae & $\begin{array}{l}\text { Dichromanthus cinnabarinus } \\
\text { (Lex.) Garay }\end{array}$ & $\begin{array}{l}\text { RLF-223, RLF-289, SRL-1155, } \\
\text { SRL-1172 }\end{array}$ & Cola de león & 3 & & 0 & & 0 & -1.1298 & 0 & -0.6711 \\
\hline 574 & Orchidaceae & $\begin{array}{l}\text { Encyclia hanburyi (Lindl.) } \\
\text { Schltr. }\end{array}$ & SRL-1519 & $\begin{array}{l}\text { Monjita morada de } \\
\text { campo }\end{array}$ & 2 & & 0 & & 0.0074 & 0.3814 & 0 & \\
\hline
\end{tabular}


Table 5 Species, Spanish common names, number of uses, percentage of families that consume it; cognitive prominence values expressed as $\mathrm{S}=$ Sutrop relative prominence index ${ }^{2}$ and biocultural importance expressed as first component value of the principal component analysis by use type (edible, medicinal, firewood, fodder, ceremonial and ornamental; distribution on vegetal types, importance ecological index value (EIVI); specie origin region, ecological status, management practices and management site with respect to species wild populations (Continued)

\begin{tabular}{|c|c|c|c|c|c|c|c|c|c|c|c|c|}
\hline 575 & Orchidaceae & Epidendrum lignosum Lex. & RJS-9, RLF-50, SRL-139 & Flor de cañada & 1 & & 0 & & 0 & -0.0825 & 0 & \\
\hline 576 & Orchidaceae & $\begin{array}{l}\text { Epidendrum longipetalum } \\
\text { A.Rich. \& Galeotti }\end{array}$ & RJS-6 & $\begin{array}{l}\text { Monjita moradita de } \\
\text { varas }\end{array}$ & 1 & & 0 & & 0 & -1.2721 & 0 & \\
\hline 577 & Orchidaceae & $\begin{array}{l}\text { Epidendrum radioferens } \\
\text { (Ames, F.T.Hubb. \& } \\
\text { C.Schweinf.) } \\
\text { Hágsater }\end{array}$ & RJS-3 & Monjita colorada & 2 & $\begin{array}{l}\text { Ornamental }=12, \\
\text { ceremonial }=85\end{array}$ & 0 & & 0.0139 & 0.8741 & 0 & \\
\hline 584 & Orchidaceae & $\begin{array}{l}\text { Euchile karwinskii (Mart.) } \\
\text { Christenson }\end{array}$ & RJS-1 & Monjita amarilla & 3 & $\begin{array}{l}\text { Ornamental }=47 \\
\text { ceremonial }=99\end{array}$ & 0 & & 0.045 & 3.5005 & 0.0017 & 2.6178 \\
\hline 578 & Orchidaceae & Govenia lagenophora Lindl. & SRL-1270 & Jarrito & 3 & & 0 & 0.1688 & 0 & -0.7946 & 0 & \\
\hline 573 & Orchidaceae & $\begin{array}{l}\text { Homalopetalum kienastii } \\
\text { (Rchb.f.) Withner }\end{array}$ & SRL-1249 & & 1 & & 0 & & 0 & -0.1783 & 0 & \\
\hline 579 & Orchidaceae & $\begin{array}{l}\text { Laelia albida Bateman ex } \\
\text { Lindl. }\end{array}$ & ERL-126 & Monjita blanca & 2 & $\begin{array}{l}\text { Ornamental }=59, \\
\text { ceremonial }=77\end{array}$ & 0 & & 0.0433 & 3.0505 & 0 & \\
\hline 580 & Orchidaceae & Laelia anceps Lindl. & SRL-1541 & Monjita morada & 2 & $\begin{array}{l}\text { Ornamental }=35, \\
\text { ceremonial }=77\end{array}$ & 0 & & 0.0497 & 2.6014 & 0 & \\
\hline 581 & Orchidaceae & Malaxis unifolia Michx. & SRL-1196 & & & & 0 & & 0 & & 0 & \\
\hline 582 & Orchidaceae & $\begin{array}{l}\text { Oncidium brachyandrum } \\
\text { Lindl. }\end{array}$ & RJS-5 & Monjita pinta amarilla & 1 & & 0 & & 0 & -1.2721 & 0 & \\
\hline 570 & Orchidaceae & $\begin{array}{l}\text { Ponthieva mexicana (A.Rich. } \\
\text { \& Galeotti) Salazar }\end{array}$ & RLF-256, RLF-267 & & & & 0 & & 0 & & 0 & \\
\hline 583 & Orchidaceae & $\begin{array}{l}\text { Prosthechea concolor (Lex.) } \\
\text { W.E.Higgins }\end{array}$ & RJS-2, SRL-1189 & Monjita pintita chiquita & 1 & & 0 & & 0 & -0.1783 & 0 & \\
\hline 585 & Orchidaceae & $\begin{array}{l}\text { Prosthechea vitellina (Lindl.) } \\
\text { W.E.Higgins }\end{array}$ & Photo record & Monjita & 1 & & 0 & & 0 & -1.2721 & 0 & \\
\hline 586 & Orchidaceae & $\begin{array}{l}\text { Rhynchostele maculata (Lex.) } \\
\text { Soto Arenas \& Salazar }\end{array}$ & ERL-173, SRL-1476 & Monjita pinta & 2 & $\begin{array}{l}\text { Ornamental =6, } \\
\text { ceremonial = } 92\end{array}$ & 0 & & 0.0174 & 0.8134 & 0 & \\
\hline 587 & Orchidaceae & Spiranthes sp. & RLF-208 & Monjita de peña & 1 & & 0 & & 0 & & 0 & \\
\hline 588 & Orchidaceae & & Photo record & Monjita & 1 & & 0 & & 0 & -0.1783 & 0 & \\
\hline 589 & Orchidaceae & & Photo record & Monjita & 1 & & 0 & & 0 & -0.1783 & 0 & \\
\hline 590 & Orchidaceae & & Photo record & $\begin{array}{l}\text { Monjita de camotito } \\
\text { largo }\end{array}$ & 1 & Ornamental $=6$ & 0 & & 0 & -0.0219 & 0 & \\
\hline 719 & Orobanchaceae & Buchnera pusilla Kunth & RLF-235 & & 1 & & 0 & & 0 & -2.1063 & 0 & \\
\hline 720 & Orobanchaceae & $\begin{array}{l}\text { Castilleja tenuifolia } \\
\text { M.Martens \& Galeotti }\end{array}$ & $\begin{array}{l}\text { SRL-117, SRL-223, SRL-329, SRL- } \\
\text { 1438, SRL-1485 }\end{array}$ & Romero cimarrón & 3 & & 0 & -0.1987 & 0 & & 0 & -0.5504 \\
\hline 591 & Orobanchaceae & Conopholis alpina Liebm. & SRL-218, SRL-1481 & Flor de elote & 2 & & 0 & -0.7655 & 0 & & 0 & -0.9186 \\
\hline
\end{tabular}


Table 5 Species, Spanish common names, number of uses, percentage of families that consume it; cognitive prominence values expressed as $\mathrm{S}=$ Sutrop relative prominence index ${ }^{2}$ and biocultural importance expressed as first component value of the principal component analysis by use type (edible, medicinal, firewood, fodder, ceremonial and ornamental; distribution on vegetal types, importance ecological index value (EIVI); specie origin region, ecological status, management practices and management site with respect to species wild populations (Continued)

\begin{tabular}{|c|c|c|c|c|c|c|c|c|c|c|c|c|}
\hline 722 & Orobanchaceae & $\begin{array}{l}\text { Lamourouxia dasyantha } \\
\text { (Cham. \& Schltdl.) W.R.Ernst }\end{array}$ & SRL-1379, SRL-1429 & Lisión & 2 & Ceremonial $=17$ & 0 & & 0.0059 & -1.2315 & 0 & \\
\hline 723 & Orobanchaceae & Lamourouxia viscosa Kunth & RLF-209, SRL-372, SRL-1292 & $\begin{array}{l}\text { Moco de pavo, flor de } \\
\text { miel }\end{array}$ & 1 & & 0 & & 0 & & 0 & \\
\hline 594 & Oxalidaceae & Oxalis corniculata L. & SRL-1534 & Coyule & & & 0 & & 0 & & 0 & \\
\hline 592 & Oxalidaceae & Oxalis aff. latifolia Kunth & ERL-75, RLF-142, SRL-148 & Coyule & 2 & Edible $=45$ & 0 & 1.1914 & 0 & & 0 & \\
\hline 593 & Oxalidaceae & $\begin{array}{l}\text { Oxalis aff. nelsonii (Small) } \\
\text { R.Knuth }\end{array}$ & SRL-1273 & Coyule & 2 & Edible $=45$ & 0 & 2.8029 & 0 & & 0 & \\
\hline 595 & Oxalidaceae & Oxalis sp. & RLF-139 & Coyule delgado & 2 & & 0 & 0.095 & 0 & & 0 & \\
\hline 596 & Papaveracea & Argemone mexicana $\mathrm{L}$. & ERL-244, RLF-180, SRL-455 & Chicalote & 3 & & 0 & & 0 & & 0 & -0.3555 \\
\hline 597 & Passifloraceae & Passiflora bryonioides Kunth & SRL-1148 & Granadilla & 1 & & 0 & & 0 & & 0 & \\
\hline 598 & Passifloraceae & Passiflora suberosa L. & SRL-444, SRL-1164, SRL-1165 & & 1 & & 0 & & 0 & & 0 & -0.8137 \\
\hline 761 & Passifloraceae & $\begin{array}{l}\text { Turnera diffusa Willd. ex } \\
\text { Schult. }\end{array}$ & SRL-1220, SRL-1356, SRL-1467 & Tamorreal & 3 & Medicinal = 5 & 0 & & 0 & & 0.037 & 2.85 \\
\hline 721 & Phrymaceae & $\begin{array}{l}\text { Berendtiella levigata (B.L.Rob. } \\
\text { \& Greenm.) Thieret }\end{array}$ & RLF-229 & Hierba de pajarito & 1 & & 0 & -1.0765 & 0 & & 0 & \\
\hline 599 & Phytolaccaceae & Phytolacca icosandra L. & RLF-236 & & 1 & & 0 & & 0 & & 0 & -1.0487 \\
\hline 600 & Pinaceae & Pinus sp. & SRL-185 & Pino, ocote & 3 & Ornamental = 47 & 0 & & 0.0331 & & 0 & \\
\hline 601 & Piperaceae & $\begin{array}{l}\text { Peperomia quadrifolia (L.) } \\
\text { Kunth }\end{array}$ & ERL-146, SRL-1404, 1430 & Verdolaga & 1 & Edible $=95$ & 0 & & 0 & & 0 & \\
\hline 602 & Piperaceae & Peperomia sp. & RJS-4 & & 1 & & 0 & -1.0765 & 0 & & 0 & \\
\hline 603 & Piperaceae & Piper auritum Kunth & ERL-59, SRL-67, SRL-418 & Hierba santa & 2 & & 0 & & 0 & & 0 & \\
\hline 717 & Plantaginaceae & Antirrhinum majus L. & Photo record & Perrito & 2 & Ornamental = 12 & 0 & & 0.0147 & & 0 & \\
\hline 718 & Plantaginaceae & Bacopa monnieri (L.) Wettst. & SRL-301, SRL-1132 & Verdolaga de agua & 3 & Edible $=5$ & 0 & -0.2864 & 0 & & 0 & -0.6047 \\
\hline 724 & Plantaginaceae & Maurandya barclaiana Lindl. & ERL-171 & & 1 & Ornamental = 18 & 0 & & 0 & -1.0904 & 0 & \\
\hline 725 & Plantaginaceae & $\begin{array}{l}\text { Penstemon barbatus (Cav.) } \\
\text { Roth }\end{array}$ & $\begin{array}{l}\text { RLF-23, RLF-49, SRL-133, SRL- } \\
\text { 464, SRL-1314 }\end{array}$ & Bandera & 2 & & 0 & & 0 & & 0 & -0.8535 \\
\hline 726 & Plantaginaceae & $\begin{array}{l}\text { Penstemon roseus (Cerv. ex } \\
\text { Sweet) G.Don }\end{array}$ & SRL-124, SRL-1405 & Bandera & 1 & & 0 & -1.0765 & 0 & & 0 & \\
\hline 604 & Plantaginaceae & Plantago major L. & SRL-419 & & & & 0 & & 0 & & 0 & \\
\hline 727 & Plantaginaceae & Russelia obtusata S.F.Blake & $\begin{array}{l}\text { RLF-263, SRL-234, SRL-342, SRL- } \\
424, \text { SRL-1494 }\end{array}$ & Bandera & 1 & & 0 & & 0 & & 0 & -0.9867 \\
\hline 728 & Plantaginaceae & Veronica persica Poir. & SRL-177, SRL-1327 & & 1 & & 0 & & 0 & & 0 & \\
\hline 729 & Plantaginaceae & & SRL-1198 & Bandera & 1 & & 0 & & 0 & & 0 & -1.0487 \\
\hline
\end{tabular}
SRL-1198
Bandera 
Table 5 Species, Spanish common names, number of uses, percentage of families that consume it; cognitive prominence values expressed as $S=$ Sutrop relative prominence index ${ }^{2}$ and biocultural importance expressed as first component value of the principal component analysis by use type (edible, medicinal, firewood, fodder, ceremonial and ornamental; distribution on vegetal types, importance ecological index value (EIVI); specie origin region, ecological status, management practices and management site with respect to species wild populations (Continued)

\begin{tabular}{|c|c|c|c|c|c|c|c|c|c|c|c|}
\hline 605 & Plumbaginaceae & Plumbago pulchella Boiss. & SRL-189, SRL-1278 & & & & 0 & & 0 & & 0 \\
\hline 606 & Poaceae & $\begin{array}{l}\text { Aegopogon cenchroides } \\
\text { Humb. \& Bonpl. ex Willd. }\end{array}$ & SRL-83 & Pasto & 2 & Fodder $=20$ & 0.1738 & -0.3545 & 0.0074 & -0.904 & 0 \\
\hline 607 & Poaceae & Aristida adscensionis L. & RLF-239, SRL-354 & Pasto & 3 & & 0.1738 & & 0.0074 & & 0 \\
\hline 608 & Poaceae & Aristida jorullensis Kunth & SRL-142 & Pasto de semilla & 2 & & 0.1738 & -0.955 & 0.0074 & -1.2392 & 0 \\
\hline 609 & Poaceae & $\begin{array}{l}\text { Aristida schiedeana Trin. \& } \\
\text { Rupr. }\end{array}$ & SRL-309 & Pasto & 2 & & 0.1738 & 1.0277 & 0.0074 & 0.5759 & 0 \\
\hline 610 & Poaceae & Arundo donax L. & ERL-147, SRL-429 & Carrizo & 4 & & 0 & & 0 & & 0 \\
\hline 611 & Poaceae & Avena fatua $\mathrm{L}$. & SRL-1546 & Avena & 1 & Fodder $=10$ & 0.1041 & & 0 & & 0 \\
\hline 612 & Poaceae & $\begin{array}{l}\text { Bouteloua curtipendula } \\
\text { (Michx.) Torr. }\end{array}$ & RLF-98, RLF-237, RLF-296 & Pasto & 2 & Fodder $=20$ & 0.1738 & -0.3545 & 0.0074 & -0.904 & 0 \\
\hline 614 & Poaceae & Chloris rufescens Lag. & RLF-99 & Pastón, cebadia, gabilla & 2 & Fodder $=20$ & 0.1738 & -0.3545 & 0.0074 & -0.904 & 0 \\
\hline 615 & Poaceae & Chloris submutica Kunth & SRL-38 & Pastón & 2 & Fodder $=20$ & 0.1738 & -0.3545 & 0.0074 & -0.904 & 0 \\
\hline 613 & Poaceae & $\begin{array}{l}\text { Chondrosum simplex (Lag.) } \\
\text { Kunth }\end{array}$ & SRL-305 & Pasto & 2 & & 0.1738 & -0.8225 & 0.0074 & -1.1081 & 0 \\
\hline 616 & Poaceae & $\begin{array}{l}\text { Cymbopogon citratus (DC.) } \\
\text { Stapf }\end{array}$ & Photo record & Té limón, té de pasto & 1 & & 0 & & 0 & & 0 \\
\hline 617 & Poaceae & $\begin{array}{l}\text { Dactyloctenium aegyptium } \\
\text { (L.) Willd. }\end{array}$ & SRL-86 & Pasto de semilla & 2 & & 0.1738 & & 0.0074 & & 0 \\
\hline 618 & Poaceae & $\begin{array}{l}\text { Digitaria bicornis (Lam.) } \\
\text { Roem. \& Schult. }\end{array}$ & SRL-312 & Pasto & 2 & & 0.1738 & & 0.0074 & & 0 \\
\hline 620 & Poaceae & Eragrostis intermedia Hitchc. & RLF-164, SRL-306 & Pasto & 2 & Fodder $=20$ & 0.1738 & -0.2115 & 0.0074 & -0.7625 & 0 \\
\hline 621 & Poaceae & $\begin{array}{l}\text { Eragrostis mexicana } \\
\text { (Hornem.) Link }\end{array}$ & SRL-84 & Pasto & 2 & Fodder $=20$ & 0.1738 & -0.3545 & 0.0074 & -0.904 & 0 \\
\hline 619 & Poaceae & $\begin{array}{l}\text { Eragrostis aff. pectinacea } \\
\text { (Michx.) Nees }\end{array}$ & SRL-85 & Pasto legítimo & 2 & Fodder $=20$ & 0.1738 & -0.3545 & 0.0074 & -0.904 & 0 \\
\hline 622 & Poaceae & $\begin{array}{l}\text { Erioneuron avenaceum } \\
\text { (Humb., Bonpl. \& Kunth) } \\
\text { Tateoka }\end{array}$ & RLF-292 & Pasto & 2 & & 0.1738 & -0.955 & 0.0074 & -1.2392 & 0 \\
\hline 623 & Poaceae & $\begin{array}{l}\text { Heteropogon contortus (L.) } \\
\text { P.Beauv. ex Roem. \& Schult. }\end{array}$ & RLF-202 & Pasto & 2 & & 0.1738 & -0.955 & 0.0074 & -1.2392 & 0 \\
\hline 624 & Poaceae & Hilaria cenchroides Kunth & SRL-281, SRL-308 & Pasto & 2 & & 0.1738 & -0.8824 & 0.0074 & -1.1673 & 0 \\
\hline 625 & Poaceae & Hordeum vulgare L. & Photo record & Cebada & 1 & Fodder $=10$ & 0.0794 & & 0 & & 0 \\
\hline 626 & Poaceae & Lasiacis sp. & SRL-1506 & Otate & 1 & & 0 & & 0.0074 & -1.9051 & 0 \\
\hline 627 & Poaceae & Lycurus phleoides Kunth & SRL-307 & Pasto & 2 & & 0.1738 & -0.8745 & 0.0074 & -1.1595 & 0 \\
\hline
\end{tabular}


Table 5 Species, Spanish common names, number of uses, percentage of families that consume it; cognitive prominence values expressed as $\mathrm{S}=$ Sutrop relative prominence index ${ }^{2}$ and biocultural importance expressed as first component value of the principal component analysis by use type (edible, medicinal, firewood, fodder, ceremonial and ornamental; distribution on vegetal types, importance ecological index value (EIVI); specie origin region, ecological status, management practices and management site with respect to species wild populations (Continued)

\begin{tabular}{|c|c|c|c|c|c|c|c|c|c|c|c|c|}
\hline 628 & Poaceae & $\begin{array}{l}\text { Muhlenbergia gigantea } \\
\text { (E.Fourn.) Hitchc. }\end{array}$ & RLF-305 & Pastón & 2 & & 0 & & 0.0074 & -1.1597 & 0 & \\
\hline 629 & Poaceae & $\begin{array}{l}\text { Muhlenbergia robusta } \\
\text { (E.Fourn.) Hitchc. }\end{array}$ & RLF-66, SRL-169 & Pastón & 2 & & 0 & & 0.0074 & -1.0966 & 0 & \\
\hline 630 & Poaceae & $\begin{array}{l}\text { Nassella tenuissima (Trin.) } \\
\text { Barkworth }\end{array}$ & RLF-258 & Pasto & 2 & & 0.1738 & -0.955 & 0.0074 & -1.2392 & 0 & \\
\hline 631 & Poaceae & Oryza sativa L. & Photo record & Arroz & 1 & & 0 & & 0 & & 0 & \\
\hline 632 & Poaceae & $\begin{array}{l}\text { Otatea acuminata (Munro) } \\
\text { C.E.Calderón \& Soderstr. }\end{array}$ & RLF-250 & Otate & 2 & & 0 & & 0 & -1.3925 & 0 & \\
\hline 638 & Poaceae & Panicum maximum Jacq. & RLF-147 & Pasto cenizo, pastón & 2 & & 0.1738 & & 0.0074 & & 0 & \\
\hline 633 & Poaceae & Phalaris canariensis L. & ERL-231 & Alpiste & 1 & & 0 & & 0 & & 0 & \\
\hline 634 & Poaceae & $\begin{array}{l}\text { Piptochaetium fimbriatum } \\
\text { (Humb., Bonpl. \& Kunth) } \\
\text { Hitchc. }\end{array}$ & RLF-137, SRL-260, SRL-413 & Pasto & 3 & & 0.1738 & -0.3887 & 0.0074 & -0.7823 & 0 & -0.5508 \\
\hline 635 & Poaceae & Setaria grisebachii E.Fourn. & RLF-231,RL-358 & Pasto de semilla & 3 & & 0.1738 & -0.4232 & 0.0074 & -0.8164 & 0 & -0.5721 \\
\hline 636 & Poaceae & Sporobolus indicus (L.) R.Br. & RLF-132 & Pastón & 3 & & 0.1738 & -0.4882 & 0.0074 & -0.8807 & 0 & \\
\hline 637 & Poaceae & Triticum aestivum L. & SRL-172 & Trigo & 2 & Edible $=95$ & 0.0573 & & 0 & & 0 & \\
\hline 639 & Poaceae & Zea mays $\mathrm{L}$. & SRL-174 & Maíz & 3 & $\begin{array}{l}\text { Fodder }=80, \\
\text { edible }=100 \\
\text { ceremonial }=1\end{array}$ & 0.3047 & & 0 & & 0 & \\
\hline 640 & Poaceae & & RLF-157 & Pasto & 3 & & 0.1738 & -0.4882 & 0.0074 & -0.8807 & 0 & \\
\hline 641 & Poaceae & & SRL-311 & Pasto de semilla & 3 & & 0.1738 & -0.3818 & 0.0074 & -0.7755 & 0 & -0.5465 \\
\hline 642 & Poaceae & & SRL-258 & Pasto & 2 & & 0.1738 & -0.7199 & 0.0074 & -1.0066 & 0 & \\
\hline 643 & Poaceae & & RLF-291 & Pasto & 2 & & 0.1738 & -0.4149 & 0.0074 & -0.705 & 0 & \\
\hline 644 & Poaceae & & RLF-316 & Pasto & 2 & & 0.1738 & -0.955 & 0.0074 & -1.2392 & 0 & \\
\hline 645 & Poaceae & & RLF-331 & Pasto & 2 & & 0.1738 & -0.955 & 0.0074 & -1.2392 & 0 & \\
\hline 646 & Poaceae & & RLF-332 & Pasto & 2 & & 0.1738 & -0.955 & 0.0074 & -1.2392 & 0 & \\
\hline 647 & Poaceae & & RLF-333 & Pasto & 2 & & 0.1738 & -0.955 & 0.0074 & -1.2392 & 0 & \\
\hline 648 & Poaceae & & SRL-394 & Pasto & 2 & & 0.1738 & -0.955 & 0.0074 & -1.2392 & 0 & \\
\hline 649 & Poaceae & & RLF-317 & Pasto & 2 & Fodder $=20$ & 0.1738 & -0.3545 & 0.0074 & -0.904 & 0 & \\
\hline 650 & Polemoniaceae & $\begin{array}{l}\text { Loeselia caerulea (Cav.) } \\
\text { G.Don }\end{array}$ & $\begin{array}{l}\text { RLF-265, SRL-96, SRL-353, SRL- } \\
\text { 1267, SRL-1282, SRL-1364, SRL- } \\
\text { 1401, SRL-1458 }\end{array}$ & & 2 & & 0 & -0.2933 & 0 & & 0 & -0.6054 \\
\hline 651 & Polygalaceae & Polygala compacta Rose & SRL-255 & & & & 0 & & 0 & & 0 & \\
\hline 652 & Polygalaceae & Polygala scoparia Kunth & RLF-224, RLF-287 & & 2 & & 0 & -0.4 & 0 & & 0 & -0.8599 \\
\hline
\end{tabular}


Table 5 Species, Spanish common names, number of uses, percentage of families that consume it; cognitive prominence values expressed as $\mathrm{S}=$ Sutrop relative prominence index ${ }^{2}$ and biocultural importance expressed as first component value of the principal component analysis by use type (edible, medicinal, firewood, fodder, ceremonial and ornamental; distribution on vegetal types, importance ecological index value (EIVI); specie origin region, ecological status, management practices and management site with respect to species wild populations (Continued)

\begin{tabular}{|c|c|c|c|c|c|c|c|c|c|c|c|c|}
\hline 653 & Polygonaceae & Rumex crispus $\mathrm{L}$. & SRL-1533 & & & & 0 & & 0 & & 0 & \\
\hline 654 & Polypodiaceae & $\begin{array}{l}\text { Pleopeltis conzattii (Weath.) } \\
\text { R.M.Tryon \& A.F.Tryon }\end{array}$ & RLF-46, SRL-135, SRL-1237 & & 1 & & 0 & -1.0765 & 0 & & 0 & \\
\hline 655 & Polypodiaceae & $\begin{array}{l}\text { Pleopeltis polylepis (Roemer } \\
\text { ex Kunze) T.Moore }\end{array}$ & SRL-1434 & & & & 0 & & 0 & & 0 & \\
\hline 656 & Polypodiaceae & Polypodium martensii Mett. & RLF-47, SRL-137, SRL-1433 & Cilandrillo & 2 & & 0 & -0.7655 & 0 & -1.4404 & 0 & \\
\hline 658 & Polypodiaceae & $\begin{array}{l}\text { Polypodium thyssanolepis } \\
\text { A.Braun ex Klotzsch }\end{array}$ & RLF-294 & Cilandrillo & & & 0 & & 0 & & 0 & \\
\hline 657 & Polypodiaceae & Polypodium sp. & SRL-352 & Cilandrillo & & & 0 & & 0 & & 0 & \\
\hline 660 & Portulacaceae & & SRL-415 & & 1 & & 0 & & 0 & & 0 & \\
\hline 661 & Primulaceae & Anagallis arvensis L. & $\begin{array}{l}\text { ERL-108, ERL-228, RLF-200, SRL- } \\
87, \text { SRL-100, SRL-1133 }\end{array}$ & $\begin{array}{l}\text { Jabonera, hierba de } \\
\text { pollo }\end{array}$ & 3 & & 0 & & 0 & & 0.0065 & \\
\hline 759 & Primulaceae & $\begin{array}{l}\text { Bonellia macrocarpa (Cav.) } \\
\text { B.Ståhl \& Källersjö }\end{array}$ & SRL-1330 & & & & 0 & & 0 & & 0 & \\
\hline 662 & Proteaceae & $\begin{array}{l}\text { Grevillea robusta A.Cunn. ex } \\
\text { R.Br. }\end{array}$ & ERL-6 & & 2 & Ornamental = 12 & 0 & & 0.0042 & & 0 & \\
\hline 663 & Pteridaceae & Adiantum capillus-veneris $\mathrm{L}$. & SRL-1518 & & & & 0 & & 0 & & 0 & \\
\hline 664 & Pteridaceae & Adiantum poiretii Wikstr. & SRL-202,SRL-427 & & 1 & & 0 & & 0 & & 0 & -0.9676 \\
\hline 665 & Pteridaceae & $\begin{array}{l}\text { Astrolepis crassifolia } \\
\text { (Houlston \& T.Moore) } \\
\text { D.M.Benham \& Windham }\end{array}$ & RLF-34, SRL-389 & & & & 0 & & 0 & & 0 & \\
\hline 666 & Pteridaceae & $\begin{array}{l}\text { Cheiloplecton rigidum (Sw.) } \\
\text { Fée }\end{array}$ & $\begin{array}{l}\text { RLF-112, RLF-213, RLF-254, SRL- } \\
1457\end{array}$ & Cilandrillo & & & 0 & & 0 & & 0 & \\
\hline 667 & Pteridaceae & Notholaena sp. & SRL-230 & & & & 0 & & 0 & & 0 & \\
\hline 668 & Pteridaceae & Pellaea sp. & RLF-185 & & & & 0 & & 0 & & 0 & \\
\hline 671 & Ranunculaceae & Anemone mexicana Kunth & $\begin{array}{l}\text { RLF-43, RLF-128, RLF-271, SRL- } \\
1240\end{array}$ & Mariposa & 2 & & 0 & -0.7655 & 0 & -1.4404 & 0 & \\
\hline 672 & Ranunculaceae & Clematis dioica $\mathrm{L}$. & SRL-303, SRL-1305 & & & & 0 & & 0 & & 0 & \\
\hline 673 & Ranunculaceae & Consolida ajacis (L.) Schur & ERL-182 & Conejito & 2 & Ceremonial $=14$ & 0 & & 0.0147 & & 0 & \\
\hline 674 & Ranunculaceae & $\begin{array}{l}\text { Delphinium bicornutum } \\
\text { Hemsl. }\end{array}$ & SRL-1200 & Conejito & 1 & Ceremonial $=8$ & 0 & & 0 & & 0 & \\
\hline 675 & Ranunculaceae & Thalictrum gibbosum Lecoy. & RLF-212, RLF-302 & Chichicasle & 1 & & 0 & & 0 & & 0 & -1.0487 \\
\hline 676 & Rhamnaceae & Condalia mexicana Schltdl. & RLF-86, SRL-457, SRL-1147 & Espino capulín & 3 & Ornamental $=29$ & 0 & & 0.0074 & 0.0446 & 0 & \\
\hline 677 & Rhamnaceae & $\begin{array}{l}\text { Ziziphus amole (Sessé \& } \\
\text { Moc.) M.C.Johnst. }\end{array}$ & SRL-1329 & Cholulo & 1 & & 0 & & 0 & & 0 & \\
\hline
\end{tabular}


Table 5 Species, Spanish common names, number of uses, percentage of families that consume it; cognitive prominence values expressed as $\mathrm{S}=$ Sutrop relative prominence index ${ }^{2}$ and biocultural importance expressed as first component value of the principal component analysis by use type (edible, medicinal, firewood, fodder, ceremonial and ornamental; distribution on vegetal types, importance ecological index value (EIVI); specie origin region, ecological status, management practices and management site with respect to species wild populations (Continued)

\begin{tabular}{|c|c|c|c|c|c|c|c|c|c|c|c|c|}
\hline 679 & Rosaceae & $\begin{array}{l}\text { Cercocarpus fothergilloides } \\
\text { Kunth }\end{array}$ & SRL-1489 & Ramoncillo & 2 & & 0 & & 0 & & 0 & \\
\hline 680 & Rosaceae & $\begin{array}{l}\text { Crataegus mexicana Moc. \& } \\
\text { Sess, ex DC }\end{array}$ & SRL-1424 & Tejocote & 1 & Edible $=35$ & 0 & & 0 & & 0 & \\
\hline 681 & Rosaceae & $\begin{array}{l}\text { Eriobotrya japonica (Thunb.) } \\
\text { Lindl. }\end{array}$ & SRL-50 & Níspero & 2 & $\begin{array}{l}\text { Ornamental }=47, \\
\text { edible }=15\end{array}$ & 0 & & 0.0042 & & 0 & \\
\hline 682 & Rosaceae & Lindleya mespiloides Kunth & SRL-1223, SRL-1493 & $\begin{array}{l}\text { Hierba de pajarito, } \\
\text { campanita grande }\end{array}$ & 2 & & 0 & & 0 & & 0 & -0.8599 \\
\hline 678 & Rosaceae & $\begin{array}{l}\text { Malacomeles denticulata } \\
\text { (Kunth) G.N.Jones }\end{array}$ & $\begin{array}{l}\text { RLF-10, RLF-243, SRL-261, SRL- } \\
338, \text { SRL-474, SRL-1257, SRL- } \\
1258\end{array}$ & Tlasisle & 4 & & 0.0121 & 1.5381 & 0 & & 0 & 0.571 \\
\hline 686 & Rosaceae & Malus domestica Borkh. & ERL-82, ERL-205, SRL-227 & Manzana & 2 & $\begin{array}{l}\text { Ornamental = 94, } \\
\text { edible }=5\end{array}$ & 0 & & 0 & & 0 & \\
\hline 683 & Rosaceae & Prunus armeniaca L. & ERL-51, ERL-198 & Chabacano & 1 & & 0 & & 0 & & 0 & \\
\hline 684 & Rosaceae & Prunus persica (L.) Batsch & SRL-226, ERL-199 & Durazno & 2 & $\begin{array}{l}\text { Ornamental }=82, \\
\text { edible }=25\end{array}$ & 0 & & 0.0098 & & 0 & \\
\hline 685 & Rosaceae & $\begin{array}{l}\text { Prunus serotina subsp. capuli } \\
\text { (Cav. ex Spreng.) McVaugh }\end{array}$ & SRL-1412 & Capulí & 1 & & 0 & & 0 & & 0 & \\
\hline 687 & Rosaceae & Rosa sp. & Photo record & Rosa & 2 & $\begin{array}{l}\text { Ornamental }=59, \\
\text { ceremonial }=14\end{array}$ & 0 & & 0.1298 & & 0 & \\
\hline 688 & Rosaceae & Rosa sp. & ERL-240 & Rosa de ramito & 2 & Ornamental $=6$ & 0 & & 0.0165 & & 0 & \\
\hline 689 & Rosaceae & $\begin{array}{l}\text { Xerospiraea hartwegiana } \\
\text { (Rydb.) Henrickson }\end{array}$ & SRL-1490 & & & & 0 & & 0 & & 0 & \\
\hline 690 & Rubiaceae & $\begin{array}{l}\text { Bouvardia longiflora (Cav.) } \\
\text { Kunth }\end{array}$ & Photo record & Huele de noche & 1 & & 0 & & 0 & & 0 & \\
\hline 691 & Rubiaceae & $\begin{array}{l}\text { Bouvardia ternifolia (Cav.) } \\
\text { Schltdl. }\end{array}$ & $\begin{array}{l}\text { RLF-41, RLF-166, SRL-262, SRL- } \\
\text { 334, SRL-1417 }\end{array}$ & Ventorilla, flor de triste & 4 & Ceremonial $=8$ & 0 & 0.4335 & 0.0294 & 0.2563 & 0 & -0.1102 \\
\hline 692 & Rubiaceae & Chiococca alba (L.) Hitchc. & $\begin{array}{l}\text { SRL-336, SRL-470, SRL-1111, } \\
\text { SRL-1331, SRL-1441 }\end{array}$ & Campanita & 3 & Ceremonial = 99 & 0 & & 0.0294 & 1.2554 & 0 & \\
\hline 693 & Rubiaceae & $\begin{array}{l}\text { Coutaportla ghiesbreghtiana } \\
\text { (Baill.) Urb. }\end{array}$ & SRL-406 & & & & 0 & & 0 & & 0 & \\
\hline 694 & Rubiaceae & $\begin{array}{l}\text { Crusea diversifolia (Kunth) } \\
\text { W.R.Anderson }\end{array}$ & $\begin{array}{l}\text { RLF-21, RLF-111, SRL-381, SRL- } \\
1181\end{array}$ & & & & 0 & & 0 & & 0 & \\
\hline 695 & Rubiaceae & Crusea sp. & RLF-136, SRL-1180 & & 1 & & 0 & & 0 & & 0 & \\
\hline 696 & Rubiaceae & $\begin{array}{l}\text { Didymaea alsinoides (Cham. } \\
\text { \& Schltdl.) Standl. }\end{array}$ & SRL-322 & & & & 0 & & 0 & & 0 & \\
\hline 697 & Rubiaceae & Galium sp. & RLF-82, RLF-280, SRL-344 & & 1 & & 0 & & 0 & & 0 & -0.9933 \\
\hline
\end{tabular}


Table 5 Species, Spanish common names, number of uses, percentage of families that consume it; cognitive prominence values expressed as $S=$ Sutrop relative prominence index ${ }^{2}$ and biocultural importance expressed as first component value of the principal component analysis by use type (edible, medicinal, firewood, fodder, ceremonial and ornamental; distribution on vegetal types, importance ecological index value (EIVI); specie origin region, ecological status, management practices and management site with respect to species wild populations (Continued)

\begin{tabular}{|c|c|c|c|c|c|c|c|c|c|c|c|c|}
\hline 698 & Rubiaceae & Randia capitata DC. & RLF-281, SRL-1208 & Limoncito de coyote & 1 & & 0 & & 0 & & 0 & -1.0487 \\
\hline 699 & Rubiaceae & Randia thurberi S.Watson & SRL-1344 & & & & 0 & & 0 & & 0 & \\
\hline 700 & Rutaceae & Casimiroa edulis La Llave & ERL-130, ERL-176 & Zapote blanco & 4 & Edible $=5$ & 0 & & 0 & & 0.021 & \\
\hline 701 & Rutaceae & $\begin{array}{l}\text { Citrus aurantiifolia (Christm.) } \\
\text { Swingle }\end{array}$ & Photo record & Limón & 3 & $\begin{array}{l}\text { Ornamental = 71, } \\
\text { medicinal }=5 \\
\text { edible }=100\end{array}$ & 0 & & 0.0189 & & 0.0046 & \\
\hline 704 & Rutaceae & Citrus maxima (Burm.) Merr. & Photo record & Toronja & 1 & Ornamental $=6$ & 0 & & 0.0147 & & 0 & \\
\hline 703 & Rutaceae & Citrus sinensis (L.) Osbeck & Photo record & Naranja & 4 & $\begin{array}{l}\text { Ornamental }=12 \\
\text { edible }=100\end{array}$ & 0 & & 0.0147 & & 0.0015 & \\
\hline 705 & Rutaceae & Citrus reticulata Blanco & Photo record & Mandarina & 1 & & 0 & & 0 & & 0 & \\
\hline 702 & Rutaceae & $\begin{array}{l}\text { Citrus × latifolia (Yu.Tanaka) } \\
\text { Yu.Tanaka }\end{array}$ & Photo record & Lima & 2 & & 0 & & 0 & & 0.0056 & \\
\hline 706 & Rutaceae & Ptelea trifoliata L. & $\begin{array}{l}\text { ERL-196, RLF-27, RLF-308, SRL- } \\
274, \text { SRL-466, SRL-467 }\end{array}$ & Hierba de zorrillo & 3 & Firewood $=100$ & 0 & & 0 & & 0.0028 & -0.2649 \\
\hline 707 & Rutaceae & Ruta chalepensis $\mathrm{L}$. & $\begin{array}{l}\text { ERL-93, ERL-127, ERL-208, ERL- } \\
\text { 241, SRL-68 }\end{array}$ & Ruda & 2 & Ornamental $=53$ & 0 & & 0 & & 0.0427 & \\
\hline 708 & Rutaceae & Zanthoxylum sp. & SRL-1221 & & & & 0 & & 0 & & 0 & \\
\hline 709 & Rutaceae & Zanthoxylum sp. & SRL-326 & Hierba de zorrillo & 1 & & 0 & & 0 & & 0 & \\
\hline 710 & Rutaceae & Zanthoxylum sp. & SRL-1348 & & 1 & & 0 & & 0 & & 0 & -1.0487 \\
\hline 394 & Salicaceae & $\begin{array}{l}\text { Neopringlea viscosa (Liebm.) } \\
\text { Rose }\end{array}$ & SRL.337 & & & & 0 & & 0 & & 0 & \\
\hline 711 & Salicaceae & Salix bonplandiana Kunth & SRL-204 & Sauce & 3 & & 0 & & 0 & & 0 & \\
\hline 779 & Santalaceae & $\begin{array}{l}\text { Phoradendron } \\
\text { reichenbachianum (Seem.) } \\
\text { Oliv. }\end{array}$ & RLF-329, SRL-1483 & Injerto & & & 0 & & 0 & & 0 & \\
\hline 780 & Santalaceae & Phoradendron sp. & ERL-180, SRL-1558 & Injerto, chahuistle & & & 0 & & 0 & & 0 & \\
\hline 781 & Santalaceae & Phoradendron sp. & RLF-228, SRL-1268 & Injerto & 2 & & 0 & -0.298 & 0 & & 0 & -0.5662 \\
\hline 712 & Sapindaceae & Dodonaea viscosa (L.) Jacq. & $\begin{array}{l}\text { RLF-30, SRL-294, SRL-473, SRL- } \\
1118, \text { ERL-189 }\end{array}$ & Cachovenado & 4 & Firewood $=100$ & 0 & & 0.0147 & 0.2881 & 0 & \\
\hline 713 & Sapindaceae & Urvillea ulmacea Kunth & SRL-1332 & & & & 0 & & 0 & & 0 & \\
\hline 715 & Sapotaceae & $\begin{array}{l}\text { Sideroxylon palmeri (Rose) } \\
\text { T.D.Penn. }\end{array}$ & ERL-219, SRL-454 & Tempesquistle & 1 & Edible $=90$ & 0 & & 0 & & 0 & \\
\hline 716 & Sapotaceae & $\begin{array}{l}\text { Sideroxylon salicifolium (L.) } \\
\text { Lam. }\end{array}$ & RLF-244 & $\begin{array}{l}\text { Tempesquitle } \\
\text { cimarrón, laurelillo }\end{array}$ & 1 & & 0 & -1.0765 & 0 & & 0 & \\
\hline 714 & Sapotaceae & $\begin{array}{l}\text { Sideroxylon capiri (A.DC.) } \\
\text { Pittier }\end{array}$ & SRL-1508 & & & & 0 & & 0 & & 0 & \\
\hline
\end{tabular}

(A) 
Table 5 Species, Spanish common names, number of uses, percentage of families that consume it; cognitive prominence values expressed as $\mathrm{S}=$ Sutrop relative prominence index ${ }^{2}$ and biocultural importance expressed as first component value of the principal component analysis by use type (edible, medicinal, firewood, fodder, ceremonial and ornamental; distribution on vegetal types, importance ecological index value (EIVI); specie origin region, ecological status, management practices and management site with respect to species wild populations (Continued)

\begin{tabular}{|c|c|c|c|c|c|c|c|c|c|c|}
\hline 730 & Selaginellaceae & $\begin{array}{l}\text { Selaginella lepidophylla } \\
\text { (Hook. \& Grev.) Spring }\end{array}$ & SRL-374, SRL-1497 & & 1 & & 0 & 0 & 0 & -1.0487 \\
\hline 731 & Simaroubaceae & Castela erecta Turpin & SRL-1382 & & & & 0 & 0 & 0 & \\
\hline 732 & Smilacaceae & $\begin{array}{l}\text { Smilax moranensis } \\
\text { M.Martens \& Galeottii }\end{array}$ & SRL-233 & & & & 0 & 0 & 0 & \\
\hline 733 & Solanaceae & Brugmansia $\times$ candida Pers. & SRL-63 & Floribundio & 2 & $\begin{array}{l}\text { Ornamental }=12, \\
\text { ceremonial }=17\end{array}$ & 0 & 0 & 0 & \\
\hline 734 & Solanaceae & Capsicum annuum L. & ERL-165, ERL-204 & $\begin{array}{l}\text { Chilar de arbolito, } \\
\text { caquita de ratón, } \\
\text { chiltepe, cuaresmeño, } \\
\text { guajillo, piquín, verde }\end{array}$ & 3 & Edible $=100$ & 0 & 0 & 0 & \\
\hline 735 & Solanaceae & $\begin{array}{l}\text { Capsicum pubescens Ruiz \& } \\
\text { Pav. }\end{array}$ & ERL-181 & Chile canario & 2 & & 0 & 0 & 0 & \\
\hline 736 & Solanaceae & Capsicum sp. & RLF-135 & & 1 & & 0 & 0 & 0 & -1.0487 \\
\hline 737 & Solanaceae & Capsicum sp. & SRL-165 & & 1 & & 0 & -1.07650 & 0 & \\
\hline 738 & Solanaceae & Datura stramonuim L. & SRL-1284 & & & & 0 & 0 & 0 & \\
\hline 739 & Solanaceae & $\begin{array}{l}\text { Jaltomata procumbens (Cav.) } \\
\text { J.L.Gentry }\end{array}$ & SRL-180, SRL-1297 & Hierba mora & 2 & & 0 & 0 & 0 & -0.6249 \\
\hline 740 & Solanaceae & $\begin{array}{l}\text { Lycianthes ciliolata } \\
\text { (M.Martens \& Galeotti) Bitter }\end{array}$ & SRL-1149 & Ojo de toro & 2 & & 0 & 0 & 0.0051 & -0.5422 \\
\hline 741 & Solanaceae & Nicotiana glauca Graham & $\begin{array}{l}\text { ERL-37, RLF-105, SRL-171, SRL- } \\
1274\end{array}$ & Gigante & 4 & $\begin{array}{l}\text { Ornamental =6, } \\
\text { firewood }=100\end{array}$ & 0 & 0 & 0.0028 & \\
\hline 742 & Solanaceae & Nicotiana tabacum L. & SRL-240 & Tabaco & 1 & & 0 & 0 & 0 & \\
\hline 743 & Solanaceae & Physalis philadelphica Lam. & $\begin{array}{l}\text { ERL-36, ERL-63, ERL-64, ERL-113, } \\
\text { RLF-312, SRL-26, SRL-1138, SRL- } \\
1298\end{array}$ & $\begin{array}{l}\text { Miltomate, tomate, } \\
\text { tomate de milpa }\end{array}$ & 2 & Edible $=100$ & 0 & 0 & 0.0069 & 1.5091 \\
\hline 744 & Solanaceae & $\begin{array}{l}\text { Solandra maxima (Moc. \& } \\
\text { Sessé ex Dunal) P.S.Green }\end{array}$ & Photo record & Copa de oro & 1 & & 0 & 0.0059 & 0 & \\
\hline 745 & Solanaceae & Solanum americanum Mill. & SRL-1234 & Ticungo & 1 & & 0 & 0 & 0 & \\
\hline 746 & Solanaceae & Solanum erianthum D.Don. & ERL-91 & Tepozán & 1 & & 0 & 0 & 0.0046 & -0.7375 \\
\hline 747 & Solanaceae & Solanum lanceolatum Cav & ERL-195 & Tepozán & 1 & & 0 & 0 & 0.0046 & -0.6538 \\
\hline 748 & Solanaceae & $\begin{array}{l}\text { Solanum lesteri Hawkes \& } \\
\text { Hjert. }\end{array}$ & RLF-151 & $\begin{array}{l}\text { Hierba del tomate } \\
\text { pinto }\end{array}$ & 1 & & 0 & 0 & 0 & \\
\hline 749 & Solanaceae & Solanum Iycopersicum L. & Photo record & Jitomate & 1 & Edible $=100$ & 0 & 0 & 0 & \\
\hline 750 & Solanaceae & Solanum rostratum Dunal & SRL-380 & Chicalote de burro & 1 & & 0 & 0 & 0 & -1.0487 \\
\hline
\end{tabular}


Table 5 Species, Spanish common names, number of uses, percentage of families that consume it; cognitive prominence values expressed as $S=$ Sutrop relative prominence index ${ }^{2}$ and biocultural importance expressed as first component value of the principal component analysis by use type (edible, medicinal, firewood, fodder, ceremonial and ornamental; distribution on vegetal types, importance ecological index value (EIVI); specie origin region, ecological status, management practices and management site with respect to species wild populations (Continued)

\begin{tabular}{|c|c|c|c|c|c|c|c|c|c|c|c|c|}
\hline 751 & Solanaceae & Solanum rudepannum Dunal & $\begin{array}{l}\text { RLF-22, RLF-95, RLF-120, RLF- } \\
\text { 275, SRL-128, SRL-302 }\end{array}$ & Tepozán & 2 & & 0 & & 0 & & 0.0046 & -0.784 \\
\hline 753 & Solanaceae & Solanum tridynamum Dunal & SRL-1361, SRL-1391 & & & & 0 & & 0 & & 0 & \\
\hline 754 & Solanaceae & Solanum tuberosum L. & Photo record & Papa & 1 & Edible $=100$ & 0 & & 0 & & 0 & \\
\hline 752 & Solanaceae & Solanum sp. & SRL-27 & & & & 0 & & 0 & & 0 & \\
\hline 756 & Sterculiaceae & Melochia sp. & SRL-1555 & & & & 0 & & 0 & & 0 & \\
\hline 659 & Talinaceae & Talinum sp. & SRL-414 & & 1 & & 0 & -1.023 & 0 & & 0 & \\
\hline 757 & Thelypteridaceae & $\begin{array}{l}\text { Thelypteris albicaulis (Fée) } \\
\text { A.R.Sm. }\end{array}$ & SRL-200 & Pojalillo & & & 0 & & 0 & & 0 & \\
\hline 758 & Thelypteridaceae & Thelypteris sp. & SRL-161, RLF-303 & & 1 & & 0 & & 0 & & 0 & \\
\hline 760 & Tropaeolaceae & Tropaeolum majus L. & $\begin{array}{l}\text { ERL-18, ERL-89, RLF-182, SRL-60, } \\
\text { SRL-196 }\end{array}$ & Mastuerzo & 3 & Ornamental = 18 & 0 & & 0.0033 & & 0 & \\
\hline 762 & Typhaceae & Typha sp. & Photo record & & & & 0 & & 0 & & 0 & \\
\hline 764 & Urticaceae & $\begin{array}{l}\text { Parietaria pensylvanica Muhl. } \\
\text { ex Willd. }\end{array}$ & ERL-73, RLF-88, RLF-266, SRL-18 & Paletaria & 1 & & 0 & & 0 & & 0.0159 & -0.5533 \\
\hline 765 & Urticaceae & Pilea microphylla (L.) Liebm. & RLF-171, SRL-1256, SRL-1309 & Pinolillo & 1 & Ornamental $=6$ & 0 & & 0 & 0.0738 & 0 & \\
\hline 766 & Urticaceae & $\begin{array}{l}\text { Urera caracasana (Jacq.) } \\
\text { Gaudich. ex Griseb. }\end{array}$ & SRL-1543 & Chichicasle & 2 & & 0 & & 0 & & 0.0031 & -0.5744 \\
\hline 768 & Verbenaceae & $\begin{array}{l}\text { Citharexylum aff. } \\
\text { bourgeauianum Greenm. }\end{array}$ & SRL-1215 & & 1 & & 0 & & 0 & -2.1063 & 0 & \\
\hline 769 & Verbenaceae & $\begin{array}{l}\text { Citharexylum tetramerum } \\
\text { Brandegee }\end{array}$ & Photo record & & & & 0 & & 0 & & 0 & \\
\hline 770 & Verbenaceae & $\begin{array}{l}\text { Glandularia elegans (Kunth) } \\
\text { Umber }\end{array}$ & $\begin{array}{l}\text { RLF-5, SRL-110, SRL-279, SRL- } \\
1326, \text { SRL-1479 }\end{array}$ & & 1 & & 0 & & 0 & & 0 & -1.0167 \\
\hline 771 & Verbenaceae & Lantana achyranthifolia Desf. & $\begin{array}{l}\text { RLF-61, RLF-62, SRL-109, SRL- } \\
152, \text { SRL-369, SRL-1296 }\end{array}$ & $\begin{array}{l}\text { Hierba buena de } \\
\text { monte }\end{array}$ & 2 & & 0 & -0.2001 & 0 & & 0 & -0.4950 \\
\hline 772 & Verbenaceae & Lantana camara L. & $\begin{array}{l}\text { RLF-91, RLF-197, SRL-115, SRL- } \\
\text { 459, SRL-1112, SRL-1154, SRL- } \\
\text { 1169, SRL-1365 }\end{array}$ & Tiundica, siete negritos & 4 & & 0.0054 & 3.3596 & 0 & 0.8495 & 0.0056 & 2.2797 \\
\hline 773 & Verbenaceae & $\begin{array}{l}\text { Lantana velutina M.Martens } \\
\text { \& Galeotti }\end{array}$ & $\begin{array}{l}\text { ERL-185, RLF-31, RLF-204, SRL- } \\
272 \text {, SRL-1115, SRL-1168 }\end{array}$ & $\begin{array}{l}\text { Tiundica blanca, cinco } \\
\text { negritos }\end{array}$ & 4 & Ornamental = 12 & 0 & & 0 & 1.484 & 0 & 2.4772 \\
\hline 774 & Verbenaceae & Lippia graveolen Kunth & & $\begin{array}{l}\text { Oreganillo, salvarreal } \\
\text { de castilla }\end{array}$ & 4 & Medicinal $=5$ & 0.0065 & 0.0052 & 0 & & 0.0069 & 0.0526 \\
\hline 775 & Verbenaceae & $\begin{array}{l}\text { Lippia oaxacana B.L.Rob. \& } \\
\text { Greenm. }\end{array}$ & $\begin{array}{l}\text { SRL-71, SRL-1378, SRL-1454, } \\
\text { SRL-1549 }\end{array}$ & Salvarreal & 2 & Medicinal $=60$ & 0 & & 0 & & 0.2636 & 10.3582 \\
\hline 776 & Verbenaceae & Priva mexicana (L.) Pers. & RLF-29 & Piojito & & & 0 & & 0 & & 0 & \\
\hline
\end{tabular}


Table 5 Species, Spanish common names, number of uses, percentage of families that consume it; cognitive prominence values expressed as $S=$ Sutrop relative prominence index ${ }^{2}$ and biocultural importance expressed as first component value of the principal component analysis by use type (edible, medicinal, firewood, fodder, ceremonial and ornamental; distribution on vegetal types, importance ecological index value (EIVI); specie origin region, ecological status, management practices and management site with respect to species wild populations (Continued)

\begin{tabular}{|c|c|c|c|c|c|c|c|c|c|c|c|}
\hline 777 & Verbenaceae & $\begin{array}{l}\text { Stachytarpheta acuminata } \\
\text { A.DC. }\end{array}$ & SRL-1380 & & & & 0 & 0 & & 0 & \\
\hline 778 & Verbenaceae & Verbena carolina L. & $\begin{array}{l}\text { RLF-93, SRL-125, SRL-173, SRL- } \\
456\end{array}$ & & 1 & & 0 & 0 & -1.5594 & 0 & \\
\hline 782 & Vitaceae & Cissus sp. & $\begin{array}{l}\text { RLF-101, RLF-173, SRL-1373, } \\
\text { SRL-1535 }\end{array}$ & Tripa de diablo & 2 & & 0 & 0 & -1.2488 & 0 & -0.6837 \\
\hline 783 & Vitaceae & Vitis vinifera $\mathrm{L}$. & SRL-54 & Uva & 2 & & 0 & 0 & & 0 & \\
\hline 27 & Xanthorrhoeaceae & Aloe vera (L.) Burm.f. & ERL-188, SRL-78 & Sábila & 5 & Ornamental = 47 & 0 & 0 & & 0.0552 & \\
\hline 82 & Xanthorrhoeaceae & Asphodelus fistulosus L. & SRL-388, SRL-1415 & & 1 & Ornamental $=6$ & 0 & 0 & & 0 & \\
\hline 83 & Xanthorrhoeaceae & Kniphofia uvaria (L.) Oken & ERL-158 & Bandera española & 2 & Ornamental = 24 & 0 & 0 & & 0 & \\
\hline 784 & Zygophyllaceae & $\begin{array}{l}\text { Morkillia mexicana (DC.) } \\
\text { Rose \& Painter }\end{array}$ & SRL-1338, SRL-1349 & & & & 0 & 0 & & 0 & \\
\hline 785 & & & & Octavillo & 1 & Ceremonial $=17$ & 0 & 0 & & 0 & \\
\hline
\end{tabular}

a Collectors name: ERL Erandi Rivera Lozoya, RLF Ricardo Lemus Fernández, RJS José Rosario Jiménez Salazar, SRL Selene Rangel Landa

${ }^{\mathrm{b}}$ Fodder plants Sutrop Index details: Number of lists $=31$; Average length of lists $=6$; Number of cited items $=65$; Total number of cited items $=195$; Number of collected lists for no new information addition $=14$. Sutrop Index rarefaction curve 1

c Ornamental plants Sutrop Index details: Number of lists = 34; Average length of lists =6; Number of cited items = 85; Total number of cited items $=200$; Number of collected lists for no new information addition = 25 . Sutrop Index rarefaction curve 2

Medicinal plants Sutrop Index details: Number of lists = 36; Average lengthof lists = 8; Number of cited items =76; Total number of cited items = 285; Number of collected lists for no new information addition = 19. Sutrop Index rarefaction curve 3

${ }^{e}$ Edible plants Sutrop Index details: Number of lists $=38$; Average length of lists $=10$; Number of cited items $=83$; Total number of cited items $=387$; Number of collected lists for no new information addition $=19$.

Sutrop Index rarefaction curve 4

${ }_{f}$ Ceremonial plants Sutrop Index details: Number of lists $=36$; Average length of lists $=5$; Number of cited items $=41$; Total number of cited items $=185$; Number of collected lists for no new information addition $=13$. Sutrop Index rarefaction curve 5

${ }^{9}$ Firewood Sutrop Index details: Number of lists $=35$; Average length of lists $=7$; Number of cited items $=39$; Total number of cited items $=244$; Number of collected lists for no new information addition $=9$. Sutrop Index rarefaction curve 6

${ }^{\mathrm{h}}$ Key to vegetation type: $A A$ Anicent settlements, Bal Urban secondary vegetation, BEA Quercus liebmanni and Quercus laeta forest, BEC Quercus urbanni forest, BEM Quercus spp.forest, BG Gallery forest (Taxodium mucronatum), $B N$ Juniperus flaccida forest, CaCe Cephalocereus colummna-trajanni shrubland, CaMy Pseudomytrocereus fulviceps shrubland, $l z$ Izotal (shrubland dominated by rosettes), Me Mexical, Pal Mescal factories, Palm Palm shrubland of Brahea dulcis, Paz grassland, SB Tropical dry forest, Sol Homegardens, TS Agricultural fields, VR Riparian vegetation

' Key to Area of Origin: AC American Continent, EAAA Europa, Asia, Africa, Australia, Ixc Ixcatlán (species with wild populations in Ixcatlán territory, and Mesoamerican area native species that have naturalized populations in Ixcatlán territory), Mex Mexico, TCV Tehuacán-Cuicatlán Valley (plants natives of VTC but in Ixcatlan only could be finding in settlements under cultivation), Uk Unknown

j: Key to Ecological Status: $D$ Domesticated, $R$ - $W$ Ruderal-Weedy, $W$ Wild

$k_{\text {: }}$ Key to Management practices: $E$ Enhancement, $F$ Forage, $G$ Gathering, P Protection, Prp Propagation, $T$ Tolerance, $T i$ Transplanting of individuals, Ur Uproot

: Key to Management site: In situ = when management take place in sites where species wild populations are distributed; ex situ = when management take place in sites out of species wild populations distribution 
Table 5 Species, Spanish common names, number of uses, percentage of families that consume it; cognitive prominence values expressed as $\mathrm{S}=$ Sutrop relative prominence index ${ }^{2}$ and biocultural importance expressed as first component value of the principal component analysis by use type (edible, medicinal, firewood, fodder, ceremonial and ornamental; distribution on vegetal types, importance ecological index value (EIVI); specie origin region, ecological status, management practices and management site with respect to species wild populations (Continued)

\begin{tabular}{|c|c|c|c|c|c|c|c|c|c|c|c|c|}
\hline \multirow[t]{2}{*}{ ID } & \multirow{2}{*}{$\begin{array}{l}\text { Edible } \\
\text { Sutrop Index } \\
\text { value }^{\mathrm{e}}\end{array}$} & \multirow[b]{2}{*}{ PC1 value } & \multicolumn{2}{|l|}{ Ceremonial } & \multicolumn{2}{|l|}{ Firewood } & \multirow{2}{*}{$\begin{array}{l}\text { Vegetation } \\
\text { type }\end{array}$} & \multirow{2}{*}{$\begin{array}{l}\text { EIVI } \\
\text { (ecological } \\
\text { importance } \\
\text { value index) }\end{array}$} & \multirow[t]{2}{*}{ Origin $^{i}$} & \multirow{2}{*}{$\begin{array}{l}\text { Ecological } \\
\text { status }\end{array}$} & \multirow{2}{*}{$\begin{array}{l}\text { Management } \\
\text { practices }^{k}\end{array}$} & \multirow{2}{*}{$\begin{array}{l}\text { Management } \\
\text { site }\end{array}$} \\
\hline & & & $\begin{array}{l}\text { Sutrop Index } \\
\text { value }^{f}\end{array}$ & PC value & $\begin{array}{l}\text { Sutrop Index } \\
\text { value }^{\mathrm{g}}\end{array}$ & PC value & & & & & & \\
\hline 1 & 0 & & 0 & & 0 & & SB & 0 & $1 \times c$ & W & & \\
\hline 2 & 0 & & 0 & & 0 & & $\mathrm{CaCe}$ & 0 & Ixc & W & & \\
\hline 3 & 0 & & 0 & & 0 & & $\mathrm{CaCe}$ & 0 & Ixc & W & & \\
\hline 4 & 0 & & 0 & & 0 & & Sol & 0.000153 & TCV & W & P, Prp & ex situ \\
\hline 5 & 0 & & 0 & & 0 & & $\mathrm{BG}, \mathrm{Pal}$ & 0 & Ixc & W & T & in situ \\
\hline 20 & 0 & & 0 & & 0 & & Sol & 0.000026 & EAAA & W & P, Prp & ex situ \\
\hline 21 & 0 & & 0 & & 0 & & Sol & 0.000026 & EAAA & W & P, Prp & ex situ \\
\hline 22 & 0 & & 0 & & 0 & & Sol & 0.000026 & EAAA & W & P, Prp & ex situ \\
\hline 28 & 0 & & 0 & & 0 & & Iz & 0 & Ixc & W & & \\
\hline 29 & 0 & & 0 & & 0 & & Bal, Sol & 0.000153 & Ixc & R-W & $F, G, T, U r$ & in situ \\
\hline 30 & 0.2516 & 2.025 & 0 & & 0 & & Bal, Sol, TS & 0.006548 & Ixc & R-W & $E, F, G, P, T, U r$ & in situ \\
\hline 31 & 0.0218 & & 0 & & 0 & & Sol & 0.000051 & EAAA & $\mathrm{D}$ & P, Prp & ex situ \\
\hline 33 & 0 & & 0.0296 & & 0 & & Sol & 0.000077 & TCV & $\mathrm{D}$ & $E, P, \operatorname{Prp}$ & ex situ \\
\hline 34 & 0 & & 0 & & 0 & & Bal, BEA, BN, Iz, Me, Palm & 0.008464 & Ixc & W & $F, G$ & in situ \\
\hline 35 & 0 & & 0 & & 0 & & Iz & 0.000784 & Ixc & W & $\mathrm{F}$ & in situ \\
\hline 36 & 0 & & 0 & & 0 & & Me & 0 & Ixc & W & & \\
\hline 26 & 0 & & 0 & & 0 & & Pal, Sol & 0.000077 & EAAA & $\mathrm{D}$ & P, Prp & ex situ \\
\hline 23 & 0 & & 0 & & 0 & & Sol & 0.000051 & EAAA & $\mathrm{D}$ & P, Prp & ex situ \\
\hline 24 & 0 & & 0 & & 0 & & Sol & 0.000153 & EAAA & $\mathrm{D}$ & P, Prp & ex situ \\
\hline 37 & 0 & & 0.0588 & & 0 & & Pal, Sol & 0.000153 & EAAA & $\mathrm{D}$ & P, Prp & ex situ \\
\hline 38 & 0 & & 0.0056 & & 0 & & Pal, Sol & 0.000128 & $A C$ & $\mathrm{D}$ & P, Prp & ex situ \\
\hline 39 & 0 & & 0 & & 0 & & Pal & 0 & Ixc & W & T & in situ \\
\hline 40 & 0 & & 0 & & 0 & & Me, Sol & 0.000026 & Ixc & W & E, P, Prp, Ti & ex situ, in situ \\
\hline 41 & 0 & & 0 & & 0 & & Me & 0 & Ixc & W & & \\
\hline 42 & 0 & & 0 & & 0.0092 & -0.5723 & $\mathrm{CaCe}, \mathrm{Me}, \mathrm{Iz}, \mathrm{Palm}$ & 0.003085 & Ixc & W & G, Prp & in situ \\
\hline 43 & 0 & -1.3811 & 0 & & 0 & & $\mathrm{CaCe}$ & 0 & Ixc & W & G & in situ \\
\hline 44 & 0 & & 0 & & 0 & & $\mathrm{BG}, \mathrm{CaCe}, \mathrm{Iz}, \mathrm{SB}, \mathrm{Pal}$, Sol & 0.000026 & Ixc & W & $F, G, T$ & in situ \\
\hline 45 & 0 & 0.06 & 0 & & 0.03 & 0.2684 & BEA, BEC, Me, Pal, SB, TS & 0.013869 & Ixc & W & $F, G, T$ & in situ \\
\hline
\end{tabular}


Table 5 Species, Spanish common names, number of uses, percentage of families that consume it; cognitive prominence values expressed as $S=$ Sutrop relative prominence index ${ }^{2}$ and biocultural importance expressed as first component value of the principal component analysis by use type (edible, medicinal, firewood, fodder, ceremonial and ornamental; distribution on vegetal types, importance ecological index value (EIVI); specie origin region, ecological status, management practices and management site with respect to species wild populations (Continued)

\begin{tabular}{|c|c|c|c|c|c|c|c|c|c|c|c|c|}
\hline 46 & 0 & -0.5044 & 0 & & 0.03 & -0.422 & BEA, BEC, Iz, Me, Pal, Palm, Sol, TS & 0.023686 & Ixc & W & $G, T$ & in situ \\
\hline 47 & 0 & -0.0476 & 0 & & 0.03 & 0.134 & $\mathrm{BEA}, \mathrm{BN}, \mathrm{Iz}, \mathrm{Me}, \mathrm{BB}, \mathrm{TS}$ & 0.017724 & Ixc & W & $F, G, T$ & in situ \\
\hline 48 & 0 & & 0 & & 0 & & Sol & 0.000026 & $A C$ & W & $\mathrm{E}, \mathrm{P}, \mathrm{T}, \mathrm{Ti}$ & ex situ \\
\hline 49 & 0.0088 & & 0 & & 0 & & Sol & 0.000026 & $A C$ & $\mathrm{D}$ & P, Prp & ex situ \\
\hline 52 & 0 & & 0.0469 & & 0 & & Sol & 0.000205 & EAAA & D & E, P, Prp, T, Ti & ex situ \\
\hline 53 & 0 & & 0 & & 0 & & Bal, BG, Sol & 0 & IXc & W & $\mathrm{T}$ & in situ \\
\hline 54 & 0.0610 & & 0 & & 0 & & Sol & 0.000205 & EAAA & $\mathrm{D}$ & E, P, Prp, T, Ti & ex situ \\
\hline 55 & 0.0075 & & 0 & & 0 & & Sol & 0 & EAAA & $\mathrm{D}$ & P, Prp & ex situ \\
\hline 56 & 0 & & 0 & & 0 & & BEA, Paz & 0.003360 & Ixc & W & G & in situ \\
\hline 57 & 0 & & 0 & & 0 & & Me & 0 & Ixc & W & G & in situ \\
\hline 58 & 0 & & 0 & & 0 & & BEA, BEC & 0.001155 & Ixc & W & G & in situ \\
\hline 59 & 0.0066 & & 0 & & 0 & & Sol & 0.000051 & EAAA & W & P, Prp & ex situ \\
\hline 60 & 0.0263 & & 0 & & 0 & & Sol & 0.000128 & EAAA & $D$ & P, Prp & ex situ \\
\hline 61 & 0 & & 0 & & 0 & & $\mathrm{NE}, \mathrm{TS}$ & 0 & Nat-Uk & W & $F, T, U r$ & ex situ \\
\hline 75 & 0 & & 0 & & 0 & & Sol & 0.000026 & Ixc & W & P, Prp & ex situ, in situ \\
\hline 76 & 0 & & 0 & & 0 & & BEA & 0 & Ixc & W & & \\
\hline 64 & 0 & & 0 & & 0 & & $\mathrm{CaCe}$ & 0 & Ixc & W & G & in situ \\
\hline 78 & 0 & & 0 & & 0 & & BEA, Sol & 0 & Ixc & W & $\mathrm{F}$ & in situ \\
\hline 79 & 0 & & 0 & & 0 & & Sol & 0.000026 & EAAA & W & P, Prp & ex situ \\
\hline 77 & 0.0022 & -0.5798 & 0 & & 0 & & BEA, Pal, Sol & 0 & Ixc & W & $G, P, T$ & in situ \\
\hline 80 & 0 & & 0 & & 0 & & Iz & 0 & Ixc & W & & \\
\hline 62 & 0 & & 0.004 & & 0 & & Sol & 0.000153 & EAAA & $D$ & P, Prp, Ti & ex situ \\
\hline 63 & 0 & & 0.007 & 0.608 & 0 & & CaMy, Sol & 0.000051 & Ixc & W & G, P, Prp & ex situ, in situ \\
\hline 81 & 0 & & 0 & & 0 & & BEA & 0 & Ixc & W & & \\
\hline 65 & 0 & & 0.12 & & 0 & & Pal, Sol & 0.000230 & EAAA & $\mathrm{D}$ & P, Prp, Ti & ex situ \\
\hline 66 & 0 & & 0 & & 0 & & BEA, Sol & 0.000026 & Ixc & W & $G, P, T$ & in situ \\
\hline 67 & 0 & & 0 & & 0 & & Sol & 0.000026 & EAAA & W & $\mathrm{P}, \mathrm{Ti}$ & ex situ \\
\hline 68 & 0.0015 & 3.3156 & 0 & 4.1723 & 0.0086 & 3.952 & BEA, BEC, BG, BN, Iz, Me, Pal, Palm, Sol, TS & 0.105714 & Ixc & W & $E, F, G, P, T, T i$ & in situ \\
\hline 69 & 0 & & 0 & -0.1118 & 0 & -0.4762 & BEA & 0 & Ixc & W & $G, P$ & in situ \\
\hline 70 & 0 & & 0 & & 0 & & BEA, Me, Sol & 0 & Ixc & W & $\mathrm{G}, \mathrm{P}, \mathrm{Ti}$ & ex situ, in situ \\
\hline 71 & 0 & & 0 & & 0 & & Sol & 0.000077 & EAAA & W & $\mathrm{P}, \operatorname{Prp}, \mathrm{T}, \mathrm{Ti}$ & ex situ \\
\hline
\end{tabular}


Table 5 Species, Spanish common names, number of uses, percentage of families that consume it; cognitive prominence values expressed as $\mathrm{S}=$ Sutrop relative prominence index ${ }^{2}$ and biocultural importance expressed as first component value of the principal component analysis by use type (edible, medicinal, firewood, fodder, ceremonial and ornamental; distribution on vegetal types, importance ecological index value (EIVI); specie origin region, ecological status, management practices and management site with respect to species wild populations (Continued)

\begin{tabular}{|c|c|c|c|c|c|c|c|c|c|c|c|c|}
\hline 72 & 0 & & 0 & & 0 & & Sol & 0.000051 & $A C$ & W & $\mathrm{P}, \operatorname{Prp}, \mathrm{T}, \mathrm{Ti}$ & ex situ \\
\hline 73 & 0 & & 0 & & 0 & & Sol & 0.000026 & EAAA & W & $\mathrm{P}, \mathrm{T}, \mathrm{Ti}$ & ex situ \\
\hline 74 & 0 & & 0 & & 0 & & Sol, TS & 0 & Ixc & W & $G, T, U r$ & in situ \\
\hline 6 & 0 & & 0 & & 0 & & Sol, TS & 0.000205 & Mex & D & P, Prp, Ti & ex situ \\
\hline 9 & 0 & & 0 & & 0 & & Me, Pal, TS & 0 & Ixc & W & $G, T$ & in situ \\
\hline 10 & 0.0148 & -0.8621 & 0 & & 0 & & $|z, P a|$ & 0.001780 & Ixc & W & $F, G$ & in situ \\
\hline 11 & 0.0717 & 3.9275 & 0 & & 0 & & BEA, Iz, Me, Pal, Palm, SB, Sol, TS & 0.020100 & Ixc & W & $E, F, G, P, \operatorname{Prp}, T, T i$ & ex situ, in situ \\
\hline 12 & 0 & 3.1267 & 0 & & 0.0104 & 3.0362 & BEA, BN, Pal, Palm, Sol, TS & 0.009780 & Ixc & W & $F, G, P, \operatorname{Prp}, T, T i$ & ex situ, in situ \\
\hline 13 & 0 & & 0 & & 0 & & BEM, Sol & 0.000026 & Ixc & W & $\mathrm{G}, \mathrm{P}, \mathrm{Ti}$ & ex situ, in situ \\
\hline 14 & 0 & & 0 & & 0 & & Me, Sol & 0 & Ixc & W & $\mathrm{G}, \mathrm{P}, \mathrm{Ti}$ & ex situ, in situ \\
\hline 15 & 0 & -1.1696 & 0 & & 0 & & Iz & 0 & Ixc & W & $F, G$ & in situ \\
\hline 16 & 0 & -1.0057 & 0 & & 0 & & Iz & 0 & Ixc & W & $F, G$ & in situ \\
\hline 17 & 0 & & 0 & & 0 & & $\mathrm{Pal}$, Sol & 0.000026 & Mex & D & P, Prp, Ti & ex situ \\
\hline 8 & 0 & & 0 & & 0 & & CaCe, Iz, Pal, SB, Sol, Ts & 0.002851 & Ixc & $D, W$ & G, P, Prp & ex situ, in situ \\
\hline 553 & 0 & & 0 & -0.75 & 0 & & Iz & 0.012638 & Ixc & W & $G, P$ & in situ \\
\hline 554 & 0.1098 & 0.1909 & 0 & -0.6392 & 0 & & $\mathrm{BG}, \mathrm{Me}$ & 0.000547 & Ixc & W & $F, G$ & in situ \\
\hline 50 & 0 & -1.0101 & 0 & & 0 & & BEA, Iz, Me & 0.003272 & Ixc & W & G & in situ \\
\hline 51 & 0 & & 0 & & 0 & & BEA, BEC & 0 & Ixc & W & $\mathrm{F}$ & in situ \\
\hline 25 & 0 & & 0 & & 0 & & Me, Palm, TS & 0 & Ixc & W & $\mathrm{T}$ & in situ \\
\hline 555 & 0 & & 0 & -1.1913 & 0 & & $\mathrm{BEA}, \mathrm{Me}$ & 0 & Ixc & W & G & in situ \\
\hline 19 & 0 & & 0 & & 0 & & AA & 0 & Ixc & W & G & in situ \\
\hline 18 & 0.0066 & & 0 & & 0 & & Sol & 0.000051 & Mex & W & P, Prp & ex situ \\
\hline 215 & 0 & & 0 & & 0 & & Sol & 0.000051 & EAAA & W & P, Prp & ex situ \\
\hline 216 & 0 & & 0 & & 0 & & Sol & 0 & EAAA & W & P, Prp & ex situ \\
\hline 217 & 0 & & 0 & & 0.0086 & -1.2037 & BEA, Iz, Me, Palm & 0.002781 & Ixc & W & $F, G$ & in situ \\
\hline 218 & 0 & & 0 & & 0 & & BEM & 0 & Ixc & W & & \\
\hline 219 & 0 & & 0 & & 0 & & Sol & 0.000051 & $A C$ & $\mathrm{D}$ & $\mathrm{P}, \mathrm{Ti}$ & ex situ \\
\hline 220 & 0 & & 0 & & 0 & & Sol & 0.000026 & EAAA & w & $\mathrm{P}, \mathrm{Ti}$ & ex situ \\
\hline 221 & 0 & & 0 & & 0 & & $\mathrm{BEA}, \mathrm{BN}, \mathrm{Iz}, \mathrm{Me}$ & 0.008107 & Ixc & W & $F, G$ & in situ \\
\hline 222 & 0 & & 0 & & 0 & & BN, Me, Palm & 0.007127 & Ixc & $R-W, W$ & G & in situ \\
\hline 223 & 0 & & 0 & & 0 & & Sol & 0 & EAAA & W & P, Prp & ex situ \\
\hline
\end{tabular}


Table 5 Species, Spanish common names, number of uses, percentage of families that consume it; cognitive prominence values expressed as $S=$ Sutrop relative prominence index ${ }^{2}$ and biocultural importance expressed as first component value of the principal component analysis by use type (edible, medicinal, firewood, fodder, ceremonial and ornamental; distribution on vegetal types, importance ecological index value (EIVI); specie origin region, ecological status, management practices and management site with respect to species wild populations (Continued)

\begin{tabular}{|c|c|c|c|c|c|c|c|c|c|c|c|c|}
\hline 224 & 0 & & 0 & & 0 & & $\mathrm{CaCe}$ & 0 & Ixc & W & $F$ & in situ \\
\hline 401 & 0 & & 0 & & 0 & & BN, Palm & 0 & Ixc & W & $F$ & in situ \\
\hline 402 & 0 & & 0 & & 0 & & Pal & 0 & Ixc & W & $\mathrm{T}$ & in situ \\
\hline 403 & 0 & & 0 & & 0 & & $\mathrm{CaCe}$ & 0 & Ixc & W & & \\
\hline 225 & 0.0075 & & 0 & & 0 & & Sol & 0.000026 & EAAA & D & P, Prp & ex situ \\
\hline 226 & 0.0038 & & 0 & & 0 & & Bal, Sol, TS & 0.002183 & Nat-EAAA & R-W & $G, T, U r$ & ex situ \\
\hline 229 & 0 & & 0 & & 0 & & Bal, Sol & 0 & Nat-EAAA & R-W & $G, T, U r$ & ex situ \\
\hline 230 & 0 & -1.1696 & 0 & & 0 & & Bal, Sol & 0 & IXc & R-W & $F, G$ & in situ \\
\hline 227 & 0 & & 0 & & 0 & & Bal, Sol, TS & 0.000026 & Nat-EAAA & R-W & $F, G, T, U r$ & ex situ \\
\hline 231 & 0 & & 0 & & 0 & & Bal, BEA, Sol & 0.000153 & Ixc & R-W & $F, G, P, T$ & in situ \\
\hline 232 & 0 & & 0.0261 & & 0 & & Sol & 0.000077 & EAAA & D & P, Prp & ex situ \\
\hline 234 & 0.0132 & & 0 & & 0 & & VR & 0 & Nat-EAAA & R-W & G & ex situ \\
\hline 233 & 0.0445 & & 0 & & 0 & & Sol & 0.000153 & EAAA & D & P, Prp & ex situ \\
\hline 235 & 0 & & 0 & & 0 & & Bal & 0 & Nat-Uk & R-W & T & ex situ \\
\hline 236 & 0 & & 0 & & 0 & & Sol & 0.000026 & $A C$ & D & P, Prp & ex situ \\
\hline 237 & 0 & & 0 & 1.1707 & 0 & & BEA, lz, Sol & 0.000026 & Ixc & W & $\mathrm{G}, \mathrm{P}, \mathrm{Ti}$ & ex situ, in situ \\
\hline 238 & 0 & & 0 & & 0 & & $\mathrm{BG}, \mathrm{Iz}, \mathrm{Me}$ & 0.002571 & Ixc & W & $F, G$ & in situ \\
\hline 239 & 0 & & 0 & & 0 & & $\mathrm{CaCe}$ & 0 & Ixc & W & & \\
\hline 240 & 0 & & 0 & -0.9895 & 0 & & Me & 0 & Ixc & W & $\mathrm{Ti}$ & in situ \\
\hline 241 & 0 & -1.0578 & 0 & & 0 & & BEA & 0 & Ixc & W & G & in situ \\
\hline 242 & 0 & & 0.0093 & 0.7779 & 0 & & $\mathrm{CaCe}, \mathrm{Me}$, Sol & 0.000026 & Ixc & W & $\mathrm{G}, \mathrm{P}, \mathrm{Ti}$ & ex situ, in situ \\
\hline 243 & 0.0044 & -0.6966 & 0 & -0.7305 & 0 & & BEM & 0 & Ixc & W & G & in situ \\
\hline 244 & 0 & & 0 & -1.1767 & 0 & & BEA, Sol & & Ixc & W & G & in situ \\
\hline 245 & 0 & 1.116 & 0 & & 0 & & BEA, Pal, Sol, VR & 0.000026 & Ixc & W & $\mathrm{G}, \mathrm{P}, \mathrm{Ti}$ & ex situ, in situ \\
\hline 246 & 0 & & 0 & & 0 & & Palm, Sol & 0.000026 & Ixc & $\mathrm{R}-\mathrm{W}$ & $G, T$ & in situ \\
\hline 247 & 0 & & 0 & 1.7881 & 0 & & BEA, BEM, Pal, Sol & 0.000128 & Ixc & W & G, P, Prp, Ti & ex situ, in situ \\
\hline 248 & 0 & & 0 & & 0 & & BEA, Sol & 0.000026 & Ixc & W & $\mathrm{G}, \mathrm{P}, \mathrm{Ti}$ & ex situ, in situ \\
\hline 249 & 0 & & 0 & 0.7241 & 0 & & BEA, Pal & 0 & Ixc & W & $\mathrm{G}, \mathrm{P}, \mathrm{Ti}$ & ex situ, in situ \\
\hline 250 & 0 & -0.894 & 0 & -0.9536 & 0 & & BEA & 0 & Ixc & W & G & in situ \\
\hline 251 & 0 & & 0 & & 0.0071 & -0.6566 & BEA, BG, Palm, Sol & 0.001533 & Ixc & W & $\mathrm{G}, \mathrm{T}$ & in situ \\
\hline 252 & 0 & & 0 & & 0 & & BEA, BN, Sol & 0.000917 & Ixc & W & $\mathrm{F}$ & in situ \\
\hline
\end{tabular}


Table 5 Species, Spanish common names, number of uses, percentage of families that consume it; cognitive prominence values expressed as $\mathrm{S}=$ Sutrop relative prominence index ${ }^{2}$ and biocultural importance expressed as first component value of the principal component analysis by use type (edible, medicinal, firewood, fodder, ceremonial and ornamental; distribution on vegetal types, importance ecological index value (EIVI); specie origin region, ecological status, management practices and management site with respect to species wild populations (Continued)

\begin{tabular}{|c|c|c|c|c|c|c|c|c|c|c|c|c|}
\hline 253 & 0 & & 0 & & 0 & & BEA & 0 & Ixc & W & & \\
\hline 254 & 0 & & 0 & & 0 & & Iz & 0 & Ixc & W & & \\
\hline 255 & 0 & & 0.0278 & 2.8995 & 0.0036 & 1.9672 & $\mathrm{Iz}, \mathrm{Me}, \mathrm{SB}$ & 0 & Ixc & W & G, P, Prp, Ti & ex situ, in situ \\
\hline 256 & 0 & & 0 & -1.1371 & 0.0036 & -1.4632 & Me & 0.000149 & Ixc & W & $F, G$ & in situ \\
\hline 257 & 0 & & 0 & & 0 & & Iz & 0 & IXc & W & & \\
\hline 258 & 0 & & 0 & & 0 & & $\mathrm{CaCe}$ & 0 & Ixc & W & & \\
\hline 259 & 0 & & 0 & -1.0042 & 0.0036 & -1.2693 & Me & 0 & Ixc & W & $G, P$ & in situ \\
\hline 260 & 0 & & 0 & & 0 & & $\mathrm{CaCe}$ & 0 & IXc & W & $\mathrm{F}$ & in situ \\
\hline 261 & 0 & & 0 & & 0 & & $\mathrm{CaCe}$ & 0 & Ixc & W & & \\
\hline 262 & 0 & & 0 & & 0 & & Sol & 0.000026 & Mex & W & P, Prp & ex situ \\
\hline 263 & 0 & & 0 & & 0 & & $\mathrm{CaCe}$, Sol & 0 & IXc & W & $\mathrm{P}, \mathrm{Ti}$ & ex situ, in situ \\
\hline 264 & 0 & & 0 & & 0 & & Me, Palm, Sol & 0.000433 & Ixc & W & $\mathrm{P}, \mathrm{Ti}$ & ex situ, in situ \\
\hline 265 & 0.0018 & & 0 & & 0 & & TS & 0 & TCV & D & Prp & ex situ \\
\hline 266 & 0.0033 & 1.0957 & 0 & & 0 & & Paz, Sol & 0.000484 & Ixc & W & $\mathrm{F}, \mathrm{P}, \mathrm{Ti}$ & ex situ, in situ \\
\hline 267 & 0.0033 & 1.4159 & 0 & & 0 & & Paz, Sol, TS & 0.001008 & Ixc & W & $\mathrm{F}, \mathrm{P}, \mathrm{T}, \mathrm{Ti}$ & ex situ, in situ \\
\hline 268 & 0.0016 & & 0 & & 0 & & Sol & 0.000153 & Mex & $D$ & P, Prp & ex situ \\
\hline 270 & 0 & & 0 & & 0 & & $\mathrm{CaCe}$ & 0 & IXc & W & & \\
\hline 271 & 0 & & 0 & 0.4819 & 0 & & BEA, Iz, Me, Palm, Sol & 0.004228 & Ixc & W & $\mathrm{P}, \mathrm{Ti}$ & ex situ, in situ \\
\hline 272 & 0 & & 0 & 0.719 & 0 & & BEA, BN, Me, Pal, Sol, TS & 0.005848 & Ixc & $R-W, W$ & $\mathrm{P}, \mathrm{T}, \mathrm{Ti}$ & ex situ, in situ \\
\hline 273 & 0 & & 0 & & 0 & & CaMy & 0 & Ixc & W & & \\
\hline 274 & 0 & & 0 & & 0 & & $\mathrm{NE}$, Sol & 0.000026 & Ixc & W & $\mathrm{P}, \mathrm{Ti}$ & ex situ, in situ \\
\hline 269 & 0 & & 0 & & 0 & & AA, Sol & 0.000256 & TCV & W & $P, \operatorname{Prp}, T, T i$ & ex situ \\
\hline 275 & 0 & & 0 & & 0 & & BEA,TS & 0 & Ixc & W & $F, G, T$ & in situ \\
\hline 276 & 0.0536 & & 0 & & 0 & & Sol & 0.000281 & Mex & $\mathrm{D}$ & P, Prp & ex situ \\
\hline 277 & 0 & & 0 & & 0 & & BEA, BEC, BN, Iz, Me, Palm, Paz, TS & 0.014065 & Ixc & $R-W, W$ & $\mathrm{~F}, \mathrm{G}, \mathrm{T}, \mathrm{Ti}$ & in situ \\
\hline 278 & 0.0433 & 2.0372 & 0 & & 0 & & Sol, TS & 0.000179 & Ixc & $R-W, W$ & P, Prp, T, Ti & ex situ, in situ \\
\hline 279 & 0 & & 0 & & 0 & & Sol & 0 & TCV & W & F, P, Prp, T, Ti & ex situ \\
\hline 280 & 0 & 2.0015 & 0 & & 0 & & Palm, Sol & 0.000026 & Ixc & W & F, Prp, T, Ti & ex situ, in situ \\
\hline 281 & 0 & & 0 & & 0 & & Palm, TS & 0 & Ixc & W & $F, P, T, T i$ & in situ \\
\hline 282 & 0 & & 0 & & 0 & & SB & 0 & Ixc & W & & \\
\hline 283 & 0 & & 0 & & 0 & & CaMy & 0 & Ixc & W & & \\
\hline
\end{tabular}


Table 5 Species, Spanish common names, number of uses, percentage of families that consume it; cognitive prominence values expressed as $\mathrm{S}=$ Sutrop relative prominence index ${ }^{2}$ and biocultural importance expressed as first component value of the principal component analysis by use type (edible, medicinal, firewood, fodder, ceremonial and ornamental; distribution on vegetal types, importance ecological index value (EIVI); specie origin region, ecological status, management practices and management site with respect to species wild populations (Continued)

\begin{tabular}{|c|c|c|c|c|c|c|c|c|c|c|}
\hline 284 & 0 & & 0 & 0 & CaMy & 0 & IXC & W & & \\
\hline 285 & 0 & & 0 & 0 & BEA & 0 & IXC & W & & \\
\hline 286 & 0 & & 0 & 0 & $\mathrm{BEA}, \mathrm{Me}, \mathrm{Pal}$ & 0 & IXC & W & $F$ & in situ \\
\hline 763 & 0 & -0.2066 & 0 & 0 & Me, Sol & 0.000179 & IXC & W & $P, T$ & in situ \\
\hline 287 & 0 & & 0 & 0 & Sol & 0.000153 & TCV & W & P, Prp & ex situ \\
\hline 288 & 0 & & 0 & 0 & $\mathrm{CaCe}$ & 0 & IXC & W & & \\
\hline 213 & 0 & & 0 & 0 & Sol & 0.000026 & EAAA & W & P, Prp & ex situ \\
\hline 767 & 0 & & 0 & 0 & VR & 0.000026 & IXC & W & F & in situ \\
\hline 289 & 0.0053 & & 0 & 0 & Sol & 0.000051 & Mex & D & P, Prp & ex situ \\
\hline 290 & 0 & & 0.0147 & 0 & Sol & 0 & EAAA & $\mathrm{D}$ & P, Prp & ex situ \\
\hline 291 & 0 & & 0 & 0.0095 & Sol & 0.000153 & EAAA & W & $\mathrm{P}, \mathrm{Ti}$ & ex situ \\
\hline 292 & 0 & & 0 & 0 & Me & 0 & IXC & W & & \\
\hline 293 & 0 & & 0 & 0 & $\mathrm{CaCe}$ & 0 & Ixc & W & & \\
\hline 294 & 0.0222 & -0.788 & 0 & 0 & Sol & 0.000026 & Ixc & R-W & $F, G, T$ & in situ \\
\hline 295 & 0.0081 & & 0 & 0 & Bal, Sol & 0.000128 & Nat-EAAA & R-W & $F, G, T, U r$ & ex situ \\
\hline 296 & 0.0237 & 0.7706 & 0 & 0 & Bal, Sol & 0.000179 & Ixc & $R-W$ & $E, P, \operatorname{Prp}, T$ & in situ \\
\hline 297 & 0.0053 & & 0 & 0 & Sol & 0 & EAAA & D & P, Prp & ex situ \\
\hline 298 & 0 & & 0 & 0 & BEA & 0 & IXC & W & & \\
\hline 299 & 0 & & 0 & 0 & BEA, Pal & 0.005276 & IxC & $R-W, W$ & F & in situ \\
\hline 300 & 0 & & 0 & 0 & Sol & 0 & EAAA & W & P, Prp & ex situ \\
\hline 301 & 0 & & 0 & 0 & $B G, I z$ & 0 & IXC & W & & \\
\hline 302 & 0 & & 0 & 0 & Sol & 0.000026 & TCV & W & P, Prp & ex situ \\
\hline 303 & 0 & & 0 & 0 & $\mathrm{Me}$ & 0 & Ixc & W & G & in situ \\
\hline 304 & 0 & & 0 & 0 & BEA, Me & 0.000920 & IXC & W & $F, G$ & in situ \\
\hline 84 & 0 & & 0 & 0 & BEA & 0 & IXC & W & & \\
\hline 85 & 0 & & 0 & 0 & $\mathrm{Me}$ & 0.004331 & Ixc & W & & \\
\hline 86 & 0 & & 0 & 0 & $\mathrm{Me}$ & 0 & IXC & W & $\mathrm{F}$ & in situ \\
\hline 87 & 0 & & 0 & 0 & BEA, BEC, BG, BN, Iz, Me, Pal, Palm, Sol, TS & 0.009661 & IxC & $R-W, W$ & $F, G, T, U r$ & in situ \\
\hline 88 & 0 & & 0 & 0 & BEA, Pal, Sol & 0.004801 & IXC & $R-W, W$ & $\mathrm{~F}, \mathrm{P}, \mathrm{T}, \mathrm{Ti}$ & ex situ, in situ \\
\hline 89 & 0 & & 0 & 0 & BEA, BG, BN, Iz, Me, Palm & 0.011943 & Ixc & $R-W, W$ & $\mathrm{~F}$ & in situ \\
\hline 90 & 0 & & 0 & 0 & Me, Sol & 0 & IXC & W & $F, G$ & in situ \\
\hline
\end{tabular}


Table 5 Species, Spanish common names, number of uses, percentage of families that consume it; cognitive prominence values expressed as $S=$ Sutrop relative prominence index ${ }^{2}$ and biocultural importance expressed as first component value of the principal component analysis by use type (edible, medicinal, firewood, fodder, ceremonial and ornamental; distribution on vegetal types, importance ecological index value (EIVI); specie origin region, ecological status, management practices and management site with respect to species wild populations (Continued)

\begin{tabular}{|c|c|c|c|c|c|c|c|c|c|c|c|}
\hline 91 & 0 & 0 & & 0 & & $\mathrm{BEA}, \mathrm{BN}, \mathrm{Me}$ & 0.003029 & IXC & W & $\mathrm{F}$ & in situ \\
\hline 92 & 0 & 0 & & 0 & & Pal, Sol & 0 & Ixc & W & $\mathrm{G}, \mathrm{T}$ & in situ \\
\hline 93 & 0 & 0 & & 0 & & BEA, BN & 0 & Ixc & W & $\mathrm{F}$ & in situ \\
\hline 94 & 0 & 0 & & 0 & & $\mathrm{BEA}, \mathrm{BN}, \mathrm{Me}, \mathrm{Paz}$ & 0.006536 & Ixc & R-W, W & G & in situ \\
\hline 97 & 0 & 0 & & 0 & & $\mathrm{BEA}, \mathrm{BEC}, \mathrm{BN}, \mathrm{Iz}$ & 0.002943 & IXC & R-W, W & $\mathrm{F}$ & in situ \\
\hline 95 & 0 & 0 & & 0 & & Sol & 0.000026 & TCV & W & P, Prp & ex situ \\
\hline 214 & 0 & 0 & & 0 & & Sol, TS & 0.008509 & Ixc & W & T & in situ \\
\hline 98 & 0 & 0 & & 0 & & BEA & 0 & Ixc & $R-W, W$ & G & in situ \\
\hline 99 & 0 & 0 & & 0.0082 & -0.2179 & BG, Pal, Palm, Sol & 0.000281 & Ixc & $R-W, W$ & $F, G, T$ & in situ \\
\hline 100 & 0 & 0 & & 0 & & VR & 0 & Ixc & W & $\mathrm{F}$ & in situ \\
\hline 101 & 0 & 0 & & 0 & & BG, Pal, Sol, TS & 0.001353 & IXC & R-W, W & $F, G, T, U r$ & in situ \\
\hline 102 & 0 & 0 & & 0 & & BEA, BEC, BG, Iz, Pal & 0.016081 & Ixc & W & $\mathrm{F}$ & in situ \\
\hline 103 & 0 & 0 & & 0 & & BEA, BN, Iz, Me, Pal, Palm, Sol, TS & 0.015409 & Ixc & $R-W, W$ & $F, G, T$ & in situ \\
\hline 104 & 0 & 0 & & 0 & & Paz & 0 & IXC & $R-W, W$ & $\mathrm{~F}$ & in situ \\
\hline 105 & 0 & 0 & & 0 & & Sol & 0.000077 & EAAA & $\mathrm{D}$ & $\mathrm{E}, \mathrm{P}, \operatorname{Prp}, \mathrm{T}, \mathrm{Ti}$ & ex situ \\
\hline 106 & 0 & 0 & & 0 & & $B E A, I z, M e$ & 0 & Ixc & W & $\mathrm{F}$ & in situ \\
\hline 107 & 0 & 0 & & 0 & & Palm & 0 & Ixc & W & $G, P$ & in situ \\
\hline 109 & 0 & 0.1021 & & 0 & & Sol & 0.000230 & EAAA & $\mathrm{D}$ & E, P, Prp, Ti & ex situ \\
\hline 110 & -1.1696 & 0 & & 0 & & $\mathrm{BG}, \mathrm{Pal}$ & 0 & IXC & W & $F, G$ & in situ \\
\hline 111 & 0 & 0 & & 0 & & BEA, BEM & 0 & Ixc & W & $\mathrm{F}$ & in situ \\
\hline 112 & 0 & 0 & & 0 & & BEA, BEC, BN, Me, Palm, Paz & 0.042091 & Ixc & $R-W, W$ & $\mathrm{~F}$ & in situ \\
\hline 113 & 0 & 0 & & 0 & & Sol & 0.000102 & Nat-Mex & W & $E, P, \operatorname{Prp}, T$ & ex situ \\
\hline 114 & 0 & 0 & 1.1027 & 0 & & BEA, BEM, Pal, Sol & 0.000051 & IXC & W & G, P, Prp, Ti & ex situ, in situ \\
\hline 115 & 0 & 0 & 1.1017 & 0 & & BEA, BEM, BG, Me, Pal, Sol & 0 & Ixc & W & G, P, Prp, Ti & ex situ, in situ \\
\hline 116 & 0 & 0.0093 & & 0 & & Sol & 0 & TCV & $\mathrm{D}$ & P, Prp & ex situ \\
\hline 117 & 0 & 0 & & 0 & & BEA & 0.006577 & Ixc & W & & \\
\hline 118 & 0 & 0 & & 0 & & Iz, Pal, Sol & 0 & IXC & $R-W$ & F & in situ \\
\hline 119 & 0 & 0 & & 0 & & BEA & 0 & IXC & W & & \\
\hline 120 & 0 & 0 & & 0 & & BEA & 0 & IXC & W & F & in situ \\
\hline 121 & 0 & 0 & & 0 & & Bal & 0 & IXC & W & T & in situ \\
\hline 122 & 0 & 0 & & 0 & & Bal, Sol & 0 & IXC & $R-W$ & $\mathrm{~T}$ & in situ \\
\hline
\end{tabular}


Table 5 Species, Spanish common names, number of uses, percentage of families that consume it; cognitive prominence values expressed as $S=$ Sutrop relative prominence index ${ }^{2}$ and biocultural importance expressed as first component value of the principal component analysis by use type (edible, medicinal, firewood, fodder, ceremonial and ornamental; distribution on vegetal types, importance ecological index value (EIVI); specie origin region, ecological status, management practices and management site with respect to species wild populations (Continued)

\begin{tabular}{|c|c|c|c|c|c|c|c|c|c|c|c|}
\hline 123 & 0 & & 0 & & 0 & BEA, Palm & 0 & $\mathrm{IXC}$ & W & $\mathrm{F}$ & in situ \\
\hline 108 & 0 & & 0.0023 & & 0 & Sol & 0.000128 & EAAA & $\mathrm{D}$ & $E, P, \operatorname{Prp}, T$ & ex situ \\
\hline 124 & 0 & & 0 & & 0 & Iz & 0 & Ixc & $R-W, W$ & & \\
\hline 125 & 0 & & 0 & & 0 & Paz & 0 & Ixc & $R-W, W$ & G & in situ \\
\hline 126 & 0 & & 0 & & 0 & Me & 0 & Ixc & W & & \\
\hline 127 & 0 & & 0 & & 0 & BEA, BN, Pal, Palm, Paz, Sol & 0.002068 & IXC & W & $G, P, \operatorname{Prp}$ & ex situ, in situ \\
\hline 128 & 0 & & 0 & & 0 & Bal, BEA, BN, Iz, Me, Pal, Palm, Sol, TS & 0.016987 & Ixc & $R-W, W$ & $F, G, T, U r$ & in situ \\
\hline 129 & 0 & & 0 & & 0 & BEA, Palm & 0 & Ixc & W & G & in situ \\
\hline 130 & 0 & & 0 & & 0 & Sol & 0.000026 & Mex & $D$ & P, Prp & ex situ \\
\hline 132 & 0.0175 & & 0 & & 0 & Sol & 0.000026 & EAAA & $D$ & P, Prp & ex situ \\
\hline 131 & 0 & & 0 & & 0 & Bal, Sol & 0 & Nat-EAAA & $\mathrm{R}-\mathrm{W}$ & F & ex situ \\
\hline 133 & 0 & & 0.0417 & & 0 & Pal, Sol & 0.000102 & EAAA & $\mathrm{D}$ & P, Prp & ex situ \\
\hline 134 & 0 & & 0 & & 0 & Sol & 0.000051 & EAAA & $D, R-W$ & E, P, Prp, T, Ti & ex situ \\
\hline 135 & 0 & & 0 & & 0 & $\mathrm{Iz}$ & 0 & IXC & W & $\mathrm{F}$ & in situ \\
\hline 136 & 0 & & 0 & & 0 & BEA, Pal & 0 & IXC & W & $\mathrm{T}, \mathrm{Ur}$ & in situ \\
\hline 137 & 0 & & 0 & & 0 & Iz & 0 & Ixc & W & & \\
\hline 138 & 0 & & 0 & & 0 & Iz, Sol & 0.000728 & Ixc & $R-W, W$ & G & in situ \\
\hline 139 & 0 & & 0 & & 0 & Iz & 0.001532 & IXC & W & $\mathrm{F}$ & in situ \\
\hline 150 & 0 & & 0 & -1.4144 & 0 & VR & 0 & IXC & W & G & in situ \\
\hline 140 & 0 & & 0 & & 0 & Sol & 0.000026 & Ixc & $R-W$ & $F, G, T, U r$ & in situ \\
\hline 141 & 0 & & 0 & & -1.7316 & $\mathrm{CaCe}, \mathrm{SB}$ & 0 & IXC & W & G & in situ \\
\hline 142 & 0 & & 0 & & 0 & Iz & 0 & IXC & W & & \\
\hline 143 & 0 & & 0 & & 0 & BEA, BEC, BN, Me, Palm, Paz, TS & 0.017574 & IXC & W & $F, T, U r$ & in situ \\
\hline 144 & 0 & & 0 & & 0 & Me & 0.001615 & Ixc & W & $\mathrm{F}$ & in situ \\
\hline 145 & 0 & & 0 & & 0 & $\mathrm{Iz}$ & 0 & Ixc & W & $F, G$ & in situ \\
\hline 146 & 0 & & 0 & & 0 & Iz & 0 & IxC & W & & \\
\hline 147 & 0 & & 0 & & 0 & Bal, BG, Iz, Paz & 0.002255 & IxC & W & G & in situ \\
\hline 148 & 0 & & 0 & & 0 & BEA & 0 & IXC & W & $F, G$ & in situ \\
\hline 151 & 0.0784 & 2.8958 & 0 & & 0 & BEA, BN, Me, Palm, Paz, Sol, TS & 0.011119 & Ixc & $R-W, W$ & G, P, Prp, T, Ti & ex situ, in situ \\
\hline 153 & 0 & & 0 & & 0 & Sol & 0 & TCV & W & $\mathrm{P}, \mathrm{Ti}$ & ex situ \\
\hline 152 & 0.1613 & 3.3603 & 0 & & 0 & Me, Sol & 0.000625 & IXC & W & E, G, P, Prp, T, Ti & ex situ, in situ \\
\hline
\end{tabular}


Table 5 Species, Spanish common names, number of uses, percentage of families that consume it; cognitive prominence values expressed as $\mathrm{S}=$ Sutrop relative prominence index ${ }^{2}$ and biocultural importance expressed as first component value of the principal component analysis by use type (edible, medicinal, firewood, fodder, ceremonial and ornamental; distribution on vegetal types, importance ecological index value (EIVI); specie origin region, ecological status, management practices and management site with respect to species wild populations (Continued)

\begin{tabular}{|c|c|c|c|c|c|c|c|c|c|c|c|c|}
\hline 154 & 0 & & 0 & & 0 & & BEA, BEC & 0.002022 & IXC & W & & \\
\hline 155 & 0 & & 0 & & 0 & & BEA, Iz & 0.001101 & IXC & W & $\mathrm{G}, \mathrm{P}, \mathrm{Ti}$ & ex situ, in situ \\
\hline 156 & 0 & & 0 & & 0 & & BEA & 0 & Ixc & W & & \\
\hline 157 & 0 & & 0 & & 0 & & BEA & 0 & IXC & W & & \\
\hline 189 & 0 & & 0 & & 0 & & BEA & 0 & IXC & W & G & in situ \\
\hline 158 & 0 & & 0 & & 0 & & Me, Palm, Sol, TS & 0.003088 & Ixc & $R-W, W$ & $F, G, T, U r$ & in situ \\
\hline 149 & 0 & & 0 & & 0 & & Me, Sol & 0.000051 & Ixc & W & $\mathrm{G}, \mathrm{P}, \mathrm{Ti}$ & ex situ, in situ \\
\hline 159 & 0 & & 0 & & 0 & & Palm, Sol, TS & 0.015309 & IXC & W & $F, G, T, U r$ & in situ \\
\hline 160 & 0 & & 0 & & 0 & & BEA, BN & 0 & IXC & W & $\mathrm{F}$ & in situ \\
\hline 161 & 0 & & 0 & & 0 & & BEA & 0 & IXC & W & $\mathrm{F}$ & in situ \\
\hline 162 & 0 & & 0 & & 0 & & Sol & 0.000102 & Nat-EAAA & R-W & $G, T, U r$ & ex situ \\
\hline 168 & 0 & & 0 & -1.1394 & 0 & & Iz, Pal, Palm & 0 & Ixc & W & $\mathrm{G}, \mathrm{T}$ & in situ \\
\hline 163 & 0 & & 0 & -1.1086 & 0 & & BN, Iz, Me, Palm, TS & 0.005100 & Ixc & R-W, W & $F, T, U r$ & in situ \\
\hline 164 & 0 & & 0 & & 0 & & Paz & 0.000463 & Ixc & W & & \\
\hline 165 & 0 & & 0 & & 0 & & BEA, BN & 0.002541 & Ixc & W & & \\
\hline 166 & 0 & & 0 & & 0 & & BN, Pal, Sol, TS & 0 & Ixc & W & $F, G, T$ & in situ \\
\hline 167 & 0 & & 0 & & 0 & & BEA, Pal & 0 & Ixc & W & G & in situ \\
\hline 169 & 0 & & 0 & -1.4144 & 0 & & $\mathrm{Me}$ & 0 & IXC & W & G & in situ \\
\hline 170 & 0 & & 0 & & 0 & & Pal & 0 & IXC & W & $F, G$ & in situ \\
\hline 96 & 0 & & 0 & & 0 & & Sol & 0.000205 & $A C$ & $R-W, W$ & E, P, Prp, T, Ti & ex situ \\
\hline 171 & 0 & & 0.3832 & & 0 & & Sol, TS & 0.000307 & TCV & $\mathrm{D}$ & E, P, Prp, T, Ti & ex situ \\
\hline 172 & 0.0053 & -0.8056 & 0.0069 & -0.4661 & 0 & & BEA, Paz & 0.003298 & Ixc & W & G & in situ \\
\hline 173 & 0 & & 0.0069 & 0.6516 & 0 & & Sol & 0.000128 & IXC & R-W, W & $E, F, P, \operatorname{Prp}, T$ & in situ \\
\hline 174 & 0 & & 0 & & 0 & & Sol & 0.000230 & EAAA & W & E, P, Prp, Ti & ex situ \\
\hline 175 & 0.0033 & & 0 & & 0 & & Sol & 0.000077 & Nat-EAAA & R-W & $G, T, U r$ & ex situ \\
\hline 176 & 0 & & 0 & & 0 & & Sol & 0.000281 & TCV & W & $E, F, P, \operatorname{Prp}$ & ex situ \\
\hline 177 & 0 & & 0 & & 0.0026 & -1.1591 & BEA, Iz, Me, Sol, TS & 0.001488 & IXC & $R-W, W$ & $F, G, T, U r$ & in situ \\
\hline 178 & 0 & & 0 & & 0 & & $\mathrm{BN}$ & 0 & IXC & $R-W, W$ & G & in situ \\
\hline 179 & 0 & & 0 & & 0.0029 & -1.668 & BEA & 0 & IxC & W & $F, G$ & in situ \\
\hline 180 & 0 & & 0 & & 0 & & Iz & 0 & IXC & W & & \\
\hline 181 & 0 & & 0 & & 0 & & Bal & 0 & Ixc & R-W, W & $\mathrm{F}$ & in situ \\
\hline
\end{tabular}


Table 5 Species, Spanish common names, number of uses, percentage of families that consume it; cognitive prominence values expressed as $S=$ Sutrop relative prominence index ${ }^{2}$ and biocultural importance expressed as first component value of the principal component analysis by use type (edible, medicinal, firewood, fodder, ceremonial and ornamental; distribution on vegetal types, importance ecological index value (EIVI); specie origin region, ecological status, management practices and management site with respect to species wild populations (Continued)

\begin{tabular}{|c|c|c|c|c|c|c|c|c|c|c|c|}
\hline 182 & 0 & -0.4176 & 0 & -0.4375 & 0 & BEA, BEC, BG, Iz, Me, Pal, Sol, TS & 0 & $\mathrm{IXC}$ & $R-W, W$ & $F, G, T, U r$ & in situ \\
\hline 183 & 0 & & 0 & & 0 & BG, Iz, Me, Pal, Palm, TS & 0 & Ixc & $R-W, W$ & $F, G, T, U r$ & in situ \\
\hline 184 & 0 & & 0 & & 0 & Iz & 0 & Ixc & $R-W, W$ & $\mathrm{~F}$ & in situ \\
\hline 185 & 0 & & 0 & & 0 & $\mathrm{I}, \mathrm{SB}$ & 0 & Ixc & W & $\mathrm{F}$ & in situ \\
\hline 186 & 0 & & 0 & & 0 & Sol & 0.000026 & Mex & $\mathrm{D}$ & P, Prp & ex situ \\
\hline 187 & 0 & & 0 & -0.6963 & 0 & BEA, BN, Iz, Me, Palm, TS & 0.009492 & IXC & $R-W, W$ & $F, G, T, U r$ & in situ \\
\hline 188 & 0 & & 0 & & 0 & BEA, BEM & 0 & Ixc & W & & \\
\hline 191 & 0 & & 0 & & 0 & Me & 0 & Ixc & W & & \\
\hline 192 & 0 & & 0 & & 0 & BEM & 0 & Ixc & W & & \\
\hline 193 & 0 & & 0 & & 0 & BG & 0 & IXC & W & & \\
\hline 194 & 0 & & 0 & -1.6375 & 0 & SB & 0 & IXC & W & G & in situ \\
\hline 195 & 0 & & 0 & & 0 & BEA & 0 & IXC & W & $\mathrm{F}$ & in situ \\
\hline 196 & 0 & & 0 & & 0 & $B G, V R$ & 0 & Ixc & W & & \\
\hline 197 & 0 & & 0 & & 0 & $\mathrm{CaCe}$ & 0 & IXC & W & G & in situ \\
\hline 198 & 0 & & 0 & & 0 & VR & 0 & IXC & W & & \\
\hline 199 & 0 & & 0 & & 0 & $\mathrm{CaCe}$ & 0 & Ixc & W & & \\
\hline 200 & 0 & & 0 & & 0 & SB & 0 & IXC & W & & \\
\hline 201 & 0 & & 0 & & 0 & BEA & 0 & Ixc & W & F & in situ \\
\hline 202 & 0 & & 0 & & 0 & SB & 0 & IXC & W & & \\
\hline 203 & 0 & & 0 & & 0 & Paz & 0 & Ixc & W & & \\
\hline 204 & 0 & & 0 & & 0 & $\mathrm{CaCe}$ & 0 & IXC & W & & \\
\hline 205 & 0 & & 0 & & 0 & $\mathrm{CaCe}$ & 0 & Ixc & W & & \\
\hline 206 & 0 & & 0 & & 0 & $\mathrm{CaCe}$ & 0 & IXC & W & & \\
\hline 207 & 0 & & 0 & & 0 & SB & 0 & IXC & W & & \\
\hline 208 & 0 & & 0 & & 0 & Me & 0 & IXC & W & & \\
\hline 209 & 0 & & 0 & & 0 & Pal, Sol, VR & 0.000026 & IXC & W & $\mathrm{G}, \mathrm{T}$ & in situ \\
\hline 210 & 0 & & 0 & & 0 & BEA & 0 & Ixc & W & G & in situ \\
\hline 211 & 0 & & 0 & & 0 & $\mathrm{CaCe}$ & 0 & IXC & W & G & in situ \\
\hline 305 & 0 & & 0 & & 0 & BEA, BN, Sol, TS & 0.000758 & IXC & $R-W$ & Ur & in situ \\
\hline 306 & 0 & & 0 & & 0 & Sol & 0 & IXC & R-W & Ur & in situ \\
\hline 307 & 0 & & 0 & & 0 & BEA, BEC, BN, Me, Palm & 0.018603 & Ixc & W & G & in situ \\
\hline
\end{tabular}


Table 5 Species, Spanish common names, number of uses, percentage of families that consume it; cognitive prominence values expressed as $S=$ Sutrop relative prominence index ${ }^{2}$ and biocultural importance expressed as first component value of the principal component analysis by use type (edible, medicinal, firewood, fodder, ceremonial and ornamental; distribution on vegetal types, importance ecological index value (EIVI); specie origin region, ecological status, management practices and management site with respect to species wild populations (Continued)

\begin{tabular}{|c|c|c|c|c|c|c|c|c|c|c|c|}
\hline 309 & 0.0042 & -1.1374 & 0 & & 0 & CaMy, Me & 0 & Ixc & W & $F, G$ & in situ \\
\hline 310 & 0 & & 0 & & 0 & BEA, Iz, Paz & 0 & Ixc & $R-W, W$ & & \\
\hline 311 & 0 & & 0 & & 0 & $\mathrm{CaCe}$ & 0 & Ixc & W & & \\
\hline 308 & 0.0042 & -1.1374 & 0 & & 0 & Me & 0 & Ixc & W & $F, G$ & in situ \\
\hline 312 & 0 & & 0 & & 0 & BEA, Me, Paz, Sol, TS & 0.000026 & IXc & $\mathrm{R}-\mathrm{W}$ & $G, T, U r$ & in situ \\
\hline 313 & 0 & & 0 & & 0 & $\mathrm{CaCe}$ & 0 & Ixc & W & $\mathrm{F}$ & in situ \\
\hline 314 & 0 & & 0 & & 0 & Sol & 0.000051 & TCV & W & Prp & ex situ \\
\hline 315 & 0 & & 0 & & 0 & Sol & 0.000026 & EAAA & W & P, Prp & ex situ \\
\hline 316 & 0 & & 0 & & 0 & Sol & 0.000051 & EAAA & W & P, Prp, T & ex situ \\
\hline 317 & 0 & & 0 & & 0 & MR, Sol & 0.000077 & Ixc & W & $\mathrm{G}, \mathrm{P}, \mathrm{Ti}$ & ex situ, in situ \\
\hline 318 & 0 & & 0 & & 0 & BEA, Me, Iz, Palm, Sol & 0.000823 & Ixc & W & $\mathrm{G}, \mathrm{P}, \mathrm{Ti}$ & ex situ, in situ \\
\hline 319 & 0 & & 0 & & 0 & Sol & 0.000026 & TCV & W & P, Prp & ex situ \\
\hline 320 & 0 & & 0 & & 0 & $\mathrm{NE}$, Sol & 0.000026 & Ixc & W & $\mathrm{P}, \mathrm{Ti}$ & ex situ, in situ \\
\hline 321 & 0 & & 0 & & 0 & Sol, VN & 0 & Ixc & W & $\mathrm{P}, \mathrm{Ti}$ & ex situ, in situ \\
\hline 322 & 0 & & 0 & & 0 & Sol & 0.000026 & EAAA & W & P, Prp & ex situ \\
\hline 323 & 0 & & 0 & & 0 & Sol & 0.000179 & EAAA & W & P, Prp & ex situ \\
\hline 324 & 0 & & 0 & & 0 & Sol & 0.000077 & TCV & W & P, Prp & ex situ \\
\hline 325 & 0 & & 0.0069 & 1.3626 & 0 & $\mathrm{NE}$, Sol & 0.000128 & Ixc & W & P, Prp, Ti & ex situ, in situ \\
\hline 326 & 0 & & 0 & & 0 & MR & 0 & Ixc & W & & \\
\hline 327 & 0 & & 0 & & 0 & BEA, BN, Me, Palm, Sol & 0.000026 & Ixc & W & F, P, Prp, Ti & ex situ, in situ \\
\hline 328 & 0 & & 0 & & 0 & Me & 0 & Ixc & W & & \\
\hline 329 & 0 & & 0 & & 0 & Sol & 0.000026 & Mex & W & P, Prp & ex situ \\
\hline 330 & 0 & & 0 & & 0 & Sol & 0.000051 & Mex & W & P, Prp & ex situ \\
\hline 331 & 0 & & 0 & & 0 & $\mathrm{MR}, \mathrm{Me}$ & 0 & Ixc & W & & \\
\hline 332 & 0 & & 0 & & 0 & Me, MR, Sol & 0.000026 & Ixc & W & F, P, Prp, Ti & ex situ, in situ \\
\hline 336 & 0 & & 0 & & 0 & Sol & 0 & EAAA & D & $P, T$ & ex situ \\
\hline 333 & 0 & & 0 & & 0 & Sol & 0.000256 & Mex & D & E, P, Prp, Ti & ex situ \\
\hline 334 & 0 & & 0 & & 0 & Bal, Pal, Sol & 0.000026 & Ixc & W & $F, G, T, U r$ & in situ \\
\hline 335 & 0.0411 & & 0 & & 0 & Sol, TS & 0.000256 & TCV & D & E, P, Prp, Ti & ex situ \\
\hline 337 & 0 & & 0 & & 0 & $\mathrm{Me}$, TS & 0 & IXc & R-W & $F, G, T, U r$ & in situ \\
\hline 338 & 0 & & 0 & & 0 & Sol, TS & 0 & Ixc & W & $F, G, T, U r$ & in situ \\
\hline
\end{tabular}


Table 5 Species, Spanish common names, number of uses, percentage of families that consume it; cognitive prominence values expressed as $\mathrm{S}=$ Sutrop relative prominence index ${ }^{2}$ and biocultural importance expressed as first component value of the principal component analysis by use type (edible, medicinal, firewood, fodder, ceremonial and ornamental; distribution on vegetal types, importance ecological index value (EIVI); specie origin region, ecological status, management practices and management site with respect to species wild populations (Continued)

\begin{tabular}{|c|c|c|c|c|c|c|c|c|c|c|c|c|}
\hline 339 & 0.0128 & & 0 & & 0 & & Sol & 0.000179 & TCV & $\mathrm{D}$ & P, Prp & ex situ \\
\hline 340 & 0 & & 0 & & 0 & & Sol, TS & 0.003422 & IxC & $\mathrm{R}-\mathrm{W}$ & $F, G, T, U r$ & in situ \\
\hline 342 & 0 & & 0 & & 0 & & Sol & 0.000102 & EAAA & W & $\mathrm{P}, \mathrm{Ti}$ & ex situ \\
\hline 341 & 0 & & 0 & & 0 & & Sol & 0.000026 & TCV & W & $\mathrm{P}, \mathrm{Ti}$ & ex situ \\
\hline 343 & 0 & & 0 & 2.7845 & 0.14 & 2.9782 & BEA, BEC, BG, BN, Iz, Me, Pal, Palm, Sol, TS & 0.085151 & IXC & W & $F, G, P, T, T i$ & in situ \\
\hline 344 & 0 & & 0 & 2.3325 & 0 & & BG, Pal, Palm, Sol & 0.018054 & IxC & W & G, P, Prp, T, Ti & ex situ, in situ \\
\hline 345 & 0 & & 0 & & 0 & & Sol & 0.000026 & $A C$ & W & $\mathrm{P}, \mathrm{Ti}$ & ex situ \\
\hline 347 & 0 & & 0 & & 0 & & Me, Palm, Paz, TS & 0.009787 & IXC & $R-W, W$ & $F, T, U r$ & in situ \\
\hline 348 & 0 & & 0 & & 0 & & $\mathrm{Me}$ & 0 & IXC & W & $F, G$ & in situ \\
\hline 349 & 0 & & 0 & & 0 & & Paz & 0.000846 & IXC & W & F & in situ \\
\hline 351 & 0 & & 0 & & 0 & & $\mathrm{Iz}$ & 0 & IXC & W & & \\
\hline 352 & 0 & & 0 & & 0 & & VR & 0 & IXC & W & G & in situ \\
\hline 353 & 0 & & 0 & & 0 & & VR & 0 & Ixc & W & G & in situ \\
\hline 346 & 0 & & 0 & & 0 & & Me, Palm, Paz, TS & 0.015465 & Ixc & W & $F, T, U r$ & in situ \\
\hline 354 & 0 & & 0 & & 0 & & BG, Pal & 0 & IXC & W & $\mathrm{F}$ & in situ \\
\hline 350 & 0 & & 0 & & 0 & & Paz & 0 & IXC & W & $\mathrm{F}$ & in situ \\
\hline 355 & 0 & & 0 & & 0 & & Paz & 0 & IXC & W & F & in situ \\
\hline 356 & 0 & -1.1696 & 0 & & 0 & & VR & 0 & IXC & W & $F, G$ & in situ \\
\hline 357 & 0 & & 0 & & 0 & & $\mathrm{Bg}$ & 0 & Ixc & W & & \\
\hline 358 & 0 & & 0 & -0.4749 & 0.12 & -0.2619 & BEA, BEC, BEM, BN, Me, TS & 0.008534 & IxC & W & $\mathrm{G}, \mathrm{T}$ & in situ \\
\hline 359 & 0 & & 0 & & 0.025 & -0.6676 & BEA, BEC, BEM, BN, Me, Palm, TS & 0.010056 & IXC & W & $\mathrm{G}, \mathrm{T}$ & in situ \\
\hline 360 & 0 & & 0 & & 0 & & BEA, Iz, Pal & 0.001362 & Ixc & W & $\mathrm{T}, \mathrm{Ur}$ & in situ \\
\hline 361 & 0 & & 0 & & 0 & & $\mathrm{CaCe}$ & 0 & IXC & W & & \\
\hline 362 & 0 & & 0 & & 0 & & Iz, Palm & 0.002686 & IXC & W & G & in situ \\
\hline 363 & 0 & & 0 & & 0 & & $\mathrm{Iz}$ & 0 & Ixc & W & & \\
\hline 364 & 0 & & 0 & & 0 & & VR & 0 & Ixc & W & & \\
\hline 365 & 0 & & 0 & & 0 & & $\mathrm{CaCe}$ & 0 & Ixc & W & $\mathrm{F}$ & in situ \\
\hline 366 & 0 & & 0 & & 0 & & $\mathrm{CaCe}$ & 0 & IXC & W & & \\
\hline 367 & 0 & & 0 & & 0 & & $\mathrm{CaCe}$ & 0 & IXC & W & & \\
\hline 368 & 0 & & 0 & & 0 & & Sol & 0.000051 & TCV & W & P, Prp & ex situ \\
\hline 369 & 0 & & 0 & & 0 & & BEA, BEC, BN, Iz, Me, Palm, Paz, TS & 0.019153 & IXC & W & $F, T, U r$ & in situ \\
\hline
\end{tabular}


Table 5 Species, Spanish common names, number of uses, percentage of families that consume it; cognitive prominence values expressed as $S=$ Sutrop relative prominence index ${ }^{2}$ and biocultural importance expressed as first component value of the principal component analysis by use type (edible, medicinal, firewood, fodder, ceremonial and ornamental; distribution on vegetal types, importance ecological index value (EIVI); specie origin region, ecological status, management practices and management site with respect to species wild populations (Continued)

\begin{tabular}{|c|c|c|c|c|c|c|c|c|c|c|c|c|}
\hline 370 & 0 & & 0 & & 0 & & BEA, Sol & 0.000026 & $\mathrm{IXC}$ & W & $G, T, U r$ & in situ \\
\hline 371 & 0 & & 0 & & 0 & & BEA, BEC, BG, Iz, Palm, Sol, TS & 0.010247 & Ixc & W & $G, T, U r$ & in situ \\
\hline 372 & 0 & & 0 & & 0 & & Sol & 0 & EAAA & W & $\mathrm{P}, \mathrm{Ti}$ & ex situ \\
\hline 373 & 0 & -1.2217 & 0 & & 0 & & Palm & 0 & IXC & W & G & in situ \\
\hline 374 & 0 & & 0.0444 & & 0 & & Sol & 0.000205 & Mex & $\mathrm{D}$ & P, Prp & ex situ \\
\hline 375 & 0 & & 0 & & 0 & & $\mathrm{CaCe}, \mathrm{Me}$ & 0 & IXC & W & & \\
\hline 376 & 0 & & 0 & & 0 & & VR & 0 & Ixc & W & & \\
\hline 377 & 0 & & 0 & & 0 & & $B E A, I z$ & 0.002724 & Ixc & W & & \\
\hline 378 & 0 & & 0 & & 0 & & $\mathrm{BN}, \mathrm{Me}$ & 0.001886 & Ixc & W & & \\
\hline 379 & 0 & & 0 & & 0 & & $\mathrm{CaCe}$ & 0 & IXC & W & $F, G$ & in situ \\
\hline 380 & 0 & & 0 & & 0 & & Bal, Sol & 0.000205 & Nat-EAAA & $\mathrm{R}-\mathrm{W}$ & $E, G, P, T, U r$ & ex situ \\
\hline 381 & 0 & & 0 & & 0 & & BEA, BN & 0.000305 & IXC & W & $\mathrm{F}$ & in situ \\
\hline 382 & 0 & & 0 & & 0 & & BEA, BEC & 0.001155 & Ixc & W & & \\
\hline 383 & 0 & & 0 & & 0 & & Iz & 0 & IXC & W & & \\
\hline 384 & 0 & & 0 & 2.1047 & 0.2789 & 2.3609 & BEM & 0 & IxC & W & $\mathrm{F}, \mathrm{G}, \mathrm{P}, \mathrm{Ti}$ & ex situ, in situ \\
\hline 385 & 0 & & 0 & 0.4695 & 0.1446 & 0.4208 & BEA, BEM, BN, TS & 0.018170 & Ixc & W & $F, G, T$ & in situ \\
\hline 386 & 0 & & 0 & 0.2552 & 0.2789 & 0.2097 & BEM & 0 & IXC & W & $F, G, P$ & in situ \\
\hline 393 & 0 & & 0 & & 0.0099 & -0.3662 & BEA, Palm, TS & 0 & IXC & W & $F, G, P, T$ & in situ \\
\hline 387 & 0 & & 0 & & 0.0155 & -0.632 & Me, Palm & 0 & IXC & W & $F, G$ & in situ \\
\hline 388 & 0 & & 0 & 3.5799 & 0.7699 & 3.806 & BEA, BEC, Pal, Sol & 0.003111 & IXC & W & $F, G, P, \operatorname{Prp}, T$ & ex situ, in situ \\
\hline 389 & 0 & & 0 & 5.4336 & 0.7699 & 5.5501 & BEA, Me, Palm, TS & 0.048434 & Ixc & W & $\mathrm{F}, \mathrm{G}, \mathrm{P}, \operatorname{Prp}, \mathrm{T}, \mathrm{Ti}$ & ex situ, in situ \\
\hline 390 & 0 & & 0 & 0.0928 & 0.1446 & 0.1359 & BEM & 0 & Ixc & W & $F, G, P$ & in situ \\
\hline 391 & 0 & & 0 & -0.5067 & 0 & -0.8204 & $\mathrm{BG}, \mathrm{Pal}$ & 0 & IXC & W & $F, G, P$ & in situ \\
\hline 392 & 0 & & 0 & 0.9619 & 0.2136 & 0.9509 & BEA, BEC, TS & 0.024545 & Ixc & W & $F, G, P, T$ & in situ \\
\hline 395 & 0 & & 0 & & 0.0222 & -0.8145 & $\mathrm{Me}, \mathrm{TS}$ & 0.010266 & Ixc & W & $\mathrm{F}, \mathrm{G}, \mathrm{T}$ & in situ \\
\hline 396 & 0 & & 0 & & 0 & & Pal, Palm & 0 & Ixc & W & T & in situ \\
\hline 397 & 0 & & 0 & & 0 & & Sol & 0.000026 & EAAA & $\mathrm{D}$ & P, Prp & ex situ \\
\hline 398 & 0 & & 0.0386 & & 0 & & Sol & 0.000384 & EAAA & $D$ & P, Prp & ex situ \\
\hline 399 & 0 & & 0 & & 0 & & Sol & 0 & IXC & W & T & in situ \\
\hline 400 & 0 & & 0.0093 & & 0 & & Sol & 0 & EAAA & D & $\mathrm{P}, \mathrm{Ti}$ & ex situ \\
\hline 404 & 0 & & 0 & & 0 & & BEA, BEC & 0.001991 & Ixc & W & $F, G$ & in situ \\
\hline
\end{tabular}


Table 5 Species, Spanish common names, number of uses, percentage of families that consume it; cognitive prominence values expressed as $S=$ Sutrop relative prominence index ${ }^{2}$ and biocultural importance expressed as first component value of the principal component analysis by use type (edible, medicinal, firewood, fodder, ceremonial and ornamental; distribution on vegetal types, importance ecological index value (EIVI); specie origin region, ecological status, management practices and management site with respect to species wild populations (Continued)

\begin{tabular}{|c|c|c|c|c|c|c|c|c|c|c|c|c|}
\hline 405 & 0 & & 0.1512 & & 0 & & Sol & 0.000179 & EAAA & $\mathrm{D}$ & P, Prp & ex situ \\
\hline 406 & 0 & & 0 & & 0 & & Pal, Sol & 0.000128 & EAAA & $\mathrm{D}$ & P, Prp & ex situ \\
\hline 407 & 0 & & 0 & & 0 & & Sol & 0 & $A C$ & $\mathrm{D}$ & P, Prp & ex situ \\
\hline 408 & 0 & & 0 & & 0 & & BEA, Iz & 0.004148 & Ixc & W & $\mathrm{F}$ & in situ \\
\hline 409 & 0 & & 0 & & 0 & & $\mathrm{Me}$ & 0 & $1 \times C$ & W & $\mathrm{F}$ & in situ \\
\hline 410 & 0 & & 0 & & 0 & & $\mathrm{Iz}$ & 0 & Ixc & W & & \\
\hline 669 & 0 & & 0 & & 0.0036 & -1.1501 & BEA, BEC, BN, Me, Palm, TS & 0.008338 & Ixc & W & $G, T$ & in situ \\
\hline 411 & 0 & & 0 & & 0 & & Sol & 0.000026 & EAAA & $\mathrm{D}$ & P, Prp & ex situ \\
\hline 412 & 0 & & 0 & & 0 & & Me, Palm & 0.002292 & Ixc & W & $F, G$ & in situ \\
\hline 413 & 0 & 0.9569 & 0 & & 0 & & BEA, Me, Pal, Sol, VR & 0.000350 & Ixc & W & $\mathrm{G}, \mathrm{P}, \mathrm{Ti}$ & ex situ, in situ \\
\hline 414 & 0 & & 0 & & 0 & & BEA & 0 & Ixc & W & & \\
\hline 415 & 0 & & 0 & & 0 & & Sol & 0 & $1 \times c$ & W & $G, T$ & in situ \\
\hline 416 & 0 & & 0 & & 0 & & Sol & 0.000026 & Nat-EAAA & R-W & $E, P, T, U r$ & ex situ \\
\hline 417 & 0 & & 0 & & 0 & & Bal, Pal, Sol & 0.000205 & Nat-EAAA & $\mathrm{R}-\mathrm{W}$ & $G, T, U r$ & ex situ \\
\hline 418 & 0.0263 & & 0 & & 0 & & Sol & 0.000358 & EAAA & $\mathrm{D}$ & P, Prp & ex situ \\
\hline 419 & 0 & & 0 & & 0 & & Sol & 0.000077 & EAAA & $\mathrm{D}$ & P, Prp, Ti & ex situ \\
\hline 420 & 0.0183 & & 0 & & 0 & & Sol & 0.000307 & EAAA & $\mathrm{D}$ & P, Prp & ex situ \\
\hline 421 & 0 & & 0 & & 0 & & Sol & 0.000026 & EAAA & W & P, Prp & ex situ \\
\hline 422 & 0 & & 0 & & 0 & & Sol & 0.000026 & EAAA & W & P, Prp & ex situ \\
\hline 424 & 0 & & 0 & & 0 & & BEA, Iz, Me, Palm & 0.004494 & Ixc & W & $\mathrm{F}$ & in situ \\
\hline 425 & 0 & & 0 & & 0 & & $\mathrm{Me}$ & 0 & Ixc & W & G & in situ \\
\hline 423 & 0 & & 0 & & 0 & & $\mathrm{Iz}, \mathrm{Palm}$ & 0 & Ixc & W & G & in situ \\
\hline 426 & 0 & & 0 & & 0 & & Palm & 0 & Ixc & W & & \\
\hline 427 & 0 & & 0 & & 0 & & BEA & 0 & Ixc & W & $F, G$ & in situ \\
\hline 428 & 0 & & 0 & & 0 & & $\mathrm{Me}, \mathrm{TS}$ & 0 & Ixc & W & $\mathrm{T}, \mathrm{Ur}$ & in situ \\
\hline 429 & 0 & & 0.0159 & -1.0238 & 0 & & BEA, BEC, BN, Iz & 0.006393 & Ixc & W & G & in situ \\
\hline 430 & 0 & & 0 & & 0 & & BEA, BEM & 0 & Ixc & W & G & in situ \\
\hline 431 & 0 & & 0 & & 0 & & $\mathrm{Iz}, \mathrm{Me}$ & 0 & Ixc & W & G & in situ \\
\hline 432 & 0 & & 0 & & 0 & & Bal, Sol, TS & 0.000179 & Ixc & W & $G, T, U r$ & in situ \\
\hline 433 & 0 & & 0 & & 0 & & $B G, B N$ & 0.001376 & Ixc & W & & \\
\hline 434 & 0 & & 0 & & 0 & & Sol & 0 & Ixc & W & $G, T$ & in situ \\
\hline
\end{tabular}


Table 5 Species, Spanish common names, number of uses, percentage of families that consume it; cognitive prominence values expressed as $\mathrm{S}=$ Sutrop relative prominence index ${ }^{2}$ and biocultural importance expressed as first component value of the principal component analysis by use type (edible, medicinal, firewood, fodder, ceremonial and ornamental; distribution on vegetal types, importance ecological index value (EIVI); specie origin region, ecological status, management practices and management site with respect to species wild populations (Continued)

\begin{tabular}{|c|c|c|c|c|c|c|c|c|c|c|c|c|}
\hline 435 & 0 & & 0 & & 0 & & BEA & 0 & IXC & W & & \\
\hline 436 & 0 & & 0 & & 0 & & Paz & 0 & IXC & W & & \\
\hline 437 & 0 & & 0 & & 0 & & BEA & 0 & Ixc & W & G & in situ \\
\hline 438 & 0 & & 0 & & 0 & & Me & 0 & Ixc & W & $\mathrm{F}$ & in situ \\
\hline 439 & 0 & & 0 & & 0 & & VR & 0 & IXC & W & & \\
\hline 440 & 0.0263 & -0.2314 & 0 & -0.5565 & 0 & & BEA & 0 & IXC & W & G, Prp & in situ \\
\hline 441 & 0.0068 & & 0 & & 0 & & Pal, Sol, TS & 0.000281 & TCV & $\mathrm{D}$ & P, Prp, T, Ti & ex situ \\
\hline 442 & 0 & & 0 & & 0 & & $\mathrm{CaCe}$ & 0 & IXC & W & $\mathrm{F}$ & in situ \\
\hline 443 & 0 & & 0 & & 0.0151 & -1.4002 & $\mathrm{NE}, \mathrm{TS}$ & 0.000647 & Ixc & W & $F, G, T$ & in situ \\
\hline 444 & 0 & & 0 & & 0.0151 & -1.164 & BEA, BEC, Iz, SB, TS & 0.014436 & IXC & W & $F, G, T$ & in situ \\
\hline 445 & 0 & & 0 & & 0.0151 & -1.2108 & Bal, Sol & 0 & Ixc & W & $\mathrm{F}, \mathrm{G}, \mathrm{T}$ & in situ \\
\hline 446 & 0 & & 0 & & 0 & & BEA & 0 & Ixc & W & $\mathrm{F}$ & in situ \\
\hline 447 & 0 & & 0 & & 0 & & BEA, Pal & 0 & IXC & W & $\mathrm{T}$ & in situ \\
\hline 448 & 0 & -1.3613 & 0 & & 0 & & $\mathrm{BEA}, \mathrm{BEC}, \mathrm{BG}, \mathrm{BN}, \mathrm{Me}$ & 0.005148 & IXC & W & $\mathrm{F}$ & in situ \\
\hline 449 & 0 & & 0 & & 0 & & $\mathrm{Me}$ & 0 & Ixc & W & & \\
\hline 450 & 0 & & 0 & & 0 & & CaMy & 0 & Ixc & W & & \\
\hline 451 & 0 & & 0 & & 0 & & BEA, Iz & 0 & IXC & W & $\mathrm{F}$ & in situ \\
\hline 452 & 0 & & 0 & & 0 & & BN & 0 & Ixc & W & $\mathrm{F}$ & in situ \\
\hline 453 & 0 & & 0 & & 0 & & Paz & 0 & IXC & W & $\mathrm{F}$ & in situ \\
\hline 454 & 0 & & 0 & & 0 & & BEA, BEC, Me & 0.010922 & Ixc & W & $\mathrm{F}$ & in situ \\
\hline 455 & 0 & & 0 & & 0 & & BN, Palm & 0 & Ixc & W & $F, G$ & in situ \\
\hline 456 & 0 & & 0 & & 0 & & VR & 0 & IxC & W & & \\
\hline 457 & 0 & & 0 & & 0 & & Me & 0 & IXC & W & & \\
\hline 458 & 0 & & 0 & & 0 & & $\mathrm{BG}, \mathrm{Iz}, \mathrm{Me}, \mathrm{TS}$ & 0 & IXC & W & $F, G, T, U r$ & in situ \\
\hline 459 & 0 & & 0 & & 0 & & VR & 0 & IXC & W & & \\
\hline 460 & 0 & & 0 & & 0 & & BN, Iz, Palm & 0.002394 & IXC & W & $F, G$ & in situ \\
\hline 461 & 0 & & 0 & & 0 & & Iz & 0 & IXc & W & F & in situ \\
\hline 462 & 0 & -1.382 & 0 & & 0 & & $\mathrm{Me}$ & 0.000310 & Ixc & W & G & in situ \\
\hline 463 & 0 & & 0 & & 0 & & $\mathrm{BN}, \mathrm{VR}$ & 0 & Ixc & W & & \\
\hline 465 & 0 & -1.3855 & 0 & & 0 & & Palm & 0 & Ixc & W & G & in situ \\
\hline 464 & 0 & -1.1696 & 0 & & 0 & & Iz & 0 & Ixc & W & $F, G$ & in situ \\
\hline
\end{tabular}


Table 5 Species, Spanish common names, number of uses, percentage of families that consume it; cognitive prominence values expressed as $S=$ Sutrop relative prominence index ${ }^{2}$ and biocultural importance expressed as first component value of the principal component analysis by use type (edible, medicinal, firewood, fodder, ceremonial and ornamental; distribution on vegetal types, importance ecological index value (EIVI); specie origin region, ecological status, management practices and management site with respect to species wild populations (Continued)

\begin{tabular}{|c|c|c|c|c|c|c|c|c|c|c|c|c|}
\hline 466 & 0 & & 0 & & 0 & & BEA, BG, BN, Me, Palm, Paz, TS & 0.014139 & IxC & W & $F, T, U r$ & in situ \\
\hline 467 & 0 & & 0 & & 0 & & $B E A, I z, M e$ & 0.000993 & Ixc & W & $\mathrm{F}$ & in situ \\
\hline 468 & 0 & & 0 & & 0 & & Me & 0 & Ixc & R-W, W & $F, G$ & in situ \\
\hline 469 & 0.0015 & & 0 & & 0 & & Sol & 0.000026 & Mex & W & $F, P, \operatorname{Prp}$ & ex situ \\
\hline 470 & 0 & & 0 & & 0.0155 & -0.3836 & BG, Iz, Me, Palm, Sol & 0.001263 & IXC & $R-W, W$ & $F, G, T$ & in situ \\
\hline 472 & 0 & & 0 & & 0 & & $\mathrm{Iz}, \mathrm{Me}$ & 0.000616 & IXC & $R-W, W$ & $\mathrm{~F}$ & in situ \\
\hline 473 & 0 & & 0 & & 0 & & Iz & 0 & IxC & $R-W, W$ & & \\
\hline 471 & 0 & & 0 & & 0 & & BEA, BEC, BG, BN, Me, Palm & 0.009509 & Ixc & $R-W, W$ & $\mathrm{~F}$ & in situ \\
\hline 474 & 0.0066 & & 0 & & 0 & & Sol & 0.000026 & EAAA & $\mathrm{D}$ & $P, T$ & ex situ \\
\hline 475 & 0.0716 & & 0 & & 0.0134 & & AA, Sol & 0.000409 & TCV & $\mathrm{D}$ & G, P, Prp, T, Ti & ex situ \\
\hline 476 & 0 & & 0 & & 0 & & Sol & 0.000205 & TCV & $\mathrm{D}$ & P, Prp, T, Ti & ex situ \\
\hline 478 & 0 & & 0 & -1.6375 & 0 & & BEA & 0 & IxC & W & G & in situ \\
\hline 477 & 0 & -1.3842 & 0 & & 0 & & BEA & 0 & Ixc & W & G & in situ \\
\hline 479 & 0 & & 0 & & 0 & & BG, Palm, Paz & 0.000841 & Ixc & W & $\mathrm{F}$ & in situ \\
\hline 480 & 0 & & 0 & & 0 & & BEA, BN, Palm, Paz & 0.000846 & Ixc & W & $\mathrm{F}$ & in situ \\
\hline 481 & 0 & & 0 & & 0 & & Sol & 0 & Nat-EAAA & $R-W$ & F & ex situ \\
\hline 482 & 0 & & 0 & & 0 & & Sol & 0 & EAAA & W & $\mathrm{F}$ & ex situ \\
\hline 483 & 0 & & 0 & & 0 & & Sol & 0 & Nat-EAAA & W & $\mathrm{F}$ & ex situ \\
\hline 484 & 0 & & 0 & & 0 & & Palm, TS & 0 & IXC & W & T & in situ \\
\hline 485 & 0 & & 0 & & 0 & & Sol & 0 & Ixc & W & $\mathrm{G}, \mathrm{T}$ & in situ \\
\hline 486 & 0 & & 0 & & 0 & & Palm, TS & 0 & Ixc & W & $\mathrm{T}, \mathrm{Ur}$ & in situ \\
\hline 487 & 0 & & 0 & & 0 & & $\mathrm{CaCe}$ & 0 & Ixc & W & & \\
\hline 488 & 0 & & 0 & & 0 & & Sol & 0.000051 & TCV & D & P, Prp & ex situ \\
\hline 489 & 0.0543 & & 0 & & 0 & & Sol, TS & 0.000230 & TCV & $D$ & P, Prp, T & ex situ \\
\hline 490 & 0 & & 0 & & 0 & & BEA & 0 & IXC & W & F & in situ \\
\hline 491 & 0 & & 0 & & 0 & & Palm, TS & 0 & IXC & W & $\mathrm{T}, \mathrm{Ur}$ & in situ \\
\hline 492 & 0 & -1.1696 & 0 & & 0 & & BEA & 0 & IXC & W & $F, G$ & in situ \\
\hline 493 & 0 & & 0 & & 0 & & BEA & 0 & IXC & W & F & in situ \\
\hline 494 & 0 & & 0 & & 0 & & SB & 0 & IXC & W & G & in situ \\
\hline 495 & 0.0219 & & 0 & & 0 & & Sol & 0 & EAAA & D & $P, T$ & ex situ \\
\hline 496 & 0.0016 & -0.6126 & 0 & & 0 & -1.016 & Pal, SB, Sol & 0.000051 & Ixc & W & $F, G, T$ & in situ \\
\hline
\end{tabular}


Table 5 Species, Spanish common names, number of uses, percentage of families that consume it; cognitive prominence values expressed as $S=$ Sutrop relative prominence index ${ }^{2}$ and biocultural importance expressed as first component value of the principal component analysis by use type (edible, medicinal, firewood, fodder, ceremonial and ornamental; distribution on vegetal types, importance ecological index value (EIVI); specie origin region, ecological status, management practices and management site with respect to species wild populations (Continued)

\begin{tabular}{|c|c|c|c|c|c|c|c|c|c|c|c|c|}
\hline 497 & 0 & & 0 & & 0 & & $B E A, I z$ & 0 & $\mathrm{IXC}$ & W & $\mathrm{F}$ & in situ \\
\hline 498 & 0 & & 0 & & 0 & & BN, Palm & 0.000623 & Ixc & W & $\mathrm{F}$ & in situ \\
\hline 499 & 0 & & 0 & -1.1062 & 0 & & Iz & 0.001468 & Ixc & W & $F, G$ & in situ \\
\hline 500 & 0 & & 0 & & 0 & & BEA, Iz, Sol & 0.000026 & Ixc & $R-W, W$ & $F, T$ & in situ \\
\hline 501 & 0 & & 0 & & 0 & & BEA, Me & 0.000420 & Ixc & W & & \\
\hline 502 & 0 & & 0 & & 0 & & BEA & 0 & Nat-Uk & W & $F, G$ & ex situ \\
\hline 503 & 0.0243 & & 0 & & 0 & & Sol, TS & 0 & EAAA & D & P, Prp & ex situ \\
\hline 504 & 0 & & 0 & & 0 & & Paz & 0.000463 & Ixc & W & $F, G$ & in situ \\
\hline 505 & 0 & & 0 & & 0.0026 & -1.0672 & BEA, BEM, Iz & 0 & Ixc & W & $F, G$ & in situ \\
\hline 506 & 0 & & 0 & & 0 & & SB & 0 & Ixc & W & $\mathrm{F}$ & in situ \\
\hline 507 & 0 & & 0 & & 0 & & $\mathrm{CaCe}$ & 0 & Ixc & W & & \\
\hline 508 & 0 & & 0 & & 0 & & Me & 0 & Ixc & W & & \\
\hline 509 & 0 & & 0 & & 0 & & Me & 0 & Ixc & W & & \\
\hline 510 & 0 & & 0 & & 0 & & BEA & 0 & Ixc & W & $\mathrm{F}$ & in situ \\
\hline 511 & 0 & & 0 & & 0 & & Sol & 0 & Ixc & W & $\mathrm{Ti}$ & ex situ, in situ \\
\hline 512 & 0 & & 0 & & 0 & & $\mathrm{CaCe}$ & 0 & Ixc & W & & \\
\hline 513 & 0 & & 0 & & 0 & & $\mathrm{CaCe}$ & 0 & Ixc & W & & \\
\hline 514 & 0 & & 0 & & 0 & & $\mathrm{CaCe}$ & 0 & Ixc & W & & \\
\hline 515 & 0 & & 0 & & 0 & & CaMy & 0 & Ixc & W & & \\
\hline 516 & 0 & & 0 & & 0 & & SB & 0 & Ixc & W & $F, G$ & in situ \\
\hline 517 & 0 & & 0 & & 0 & & BG, Me, Palm & 0.001489 & Ixc & W & & \\
\hline 518 & 0 & & 0 & & 0 & & Me & 0 & Ixc & W & & \\
\hline 519 & 0 & & 0 & & 0 & & Palm, TS & 0 & Ixc & W & $F, G, T, U r$ & in situ \\
\hline 520 & 0 & & 0 & & 0 & & $\mathrm{Bal}, \mathrm{BEA}, \mathrm{BG}$ & 0 & Ixc & W & $G, T, U r$ & in situ \\
\hline 521 & 0 & & 0 & & 0 & & CaMy, Sol, TS & 0 & Ixc & W & Ur & in situ \\
\hline 522 & 0 & & 0 & -0.8814 & 0 & & Me, Sol, Palm, TS & 0.000112 & Ixc & W & $F, G, T, U r$ & in situ \\
\hline 523 & 0 & & 0 & -1.4276 & 0 & & BN, Palm, Sol & 0.010633 & Ixc & W & G & in situ \\
\hline 524 & 0 & & 0 & -1.563 & 0 & & BEA, BN, Paz & 0.003757 & Ixc & W & G & in situ \\
\hline 670 & 0.0129 & & 0 & & 0 & & Sol & 0.000307 & EAAA & D & E, P, Prp, T, Ti & ex situ \\
\hline 525 & 0 & -1.0654 & 0 & & 0 & & Sol & 0 & Ixc & W & $G, T$ & in situ \\
\hline 526 & 0 & & 0 & & 0 & & $\mathrm{CaCe}$ & 0 & IXC & W & & \\
\hline
\end{tabular}


Table 5 Species, Spanish common names, number of uses, percentage of families that consume it; cognitive prominence values expressed as $\mathrm{S}=$ Sutrop relative prominence index ${ }^{2}$ and biocultural importance expressed as first component value of the principal component analysis by use type (edible, medicinal, firewood, fodder, ceremonial and ornamental; distribution on vegetal types, importance ecological index value (EIVI); specie origin region, ecological status, management practices and management site with respect to species wild populations (Continued)

\begin{tabular}{|c|c|c|c|c|c|c|c|c|c|c|c|c|}
\hline 527 & 0 & & 0 & & 0 & & SB & 0 & $\mid x C$ & W & & \\
\hline 528 & 0 & & 0 & -1.3063 & 0 & & BEA, BEC, Iz, Me, Palm & 0.002876 & Ixc & W & $F, G$ & in situ \\
\hline 529 & 0 & & 0 & & 0 & & Iz & 0 & Ixc & W & G & in situ \\
\hline 530 & 0 & & 0 & & 0 & & $\mathrm{CaCe}$ & 0 & IXC & W & & \\
\hline 531 & 0 & -0.6124 & 0 & & 0 & & Me, Palm, TS & 0.001293 & IXC & W & $F, G, T$ & in situ \\
\hline 532 & 0 & & 0 & & 0 & & Sol & 0.000128 & EAAA & $\mathrm{D}$ & P, Prp & ex situ \\
\hline 533 & 0.0117 & -0.4905 & 0 & & 0 & & Bal, BEA, Pal, Sol, TS & 0.000026 & Ixc & R-W & $F, G, T, U r$ & in situ \\
\hline 534 & 0 & & 0 & & 0 & & Sol & 0 & TCV & $\mathrm{D}$ & P, Prp & ex situ \\
\hline 755 & 0 & & 0 & & 0 & & Me & 0 & Ixc & W & & \\
\hline 535 & 0 & & 0 & & 0 & & Sol & 0.000026 & EAAA & $\mathrm{D}$ & $\mathrm{P}, \mathrm{Ti}$ & ex situ \\
\hline 536 & 0 & & 0 & & 0 & & Sol & 0 & EAAA & W & $\mathrm{P}, \mathrm{Ti}$ & ex situ \\
\hline 537 & 0 & & 0 & & 0 & & Bal, Sol, TS & 0.000205 & Nat-EAAA & $R-W$ & $F, G, T, U r$ & ex situ \\
\hline 538 & 0 & & 0 & & 0 & & Sol & 0.000051 & EAAA & W & P, Prp, Ti & ex situ \\
\hline 539 & 0 & & 0 & & 0 & & Bal, Sol & 0 & IXC & W & $\mathrm{F}$ & in situ \\
\hline 540 & 0.0038 & -1.148 & 0 & & 0 & & Bal, Palm, Sol, TS & 0.000026 & IxC & W & $\mathrm{G}, \mathrm{T}$ & in situ \\
\hline 541 & 0 & & 0 & & 0 & & Sol & 0.000077 & Mex & W & $\mathrm{P}, \mathrm{Ti}$ & ex situ \\
\hline 542 & 0 & & 0 & & 0 & & Sol & 0.000026 & EAAA & W & $\mathrm{P}, \mathrm{Ti}$ & ex situ \\
\hline 543 & 0 & & 0 & 0.501 & 0 & & BEA, BM, Sol & 0 & Ixc & W & $\mathrm{G}, \mathrm{P}, \mathrm{Ti}$ & ex situ, in situ \\
\hline 544 & 0 & & 0 & & 0 & & Sol & 0 & EAAA & W & $\mathrm{P}, \mathrm{Ti}$ & ex situ \\
\hline 545 & 0.0219 & & 0 & & 0 & & Sol & 0.000179 & EAAA & $D$ & P, Prp, Ti & ex situ \\
\hline 546 & 0 & -0.8491 & 0 & & 0 & & Sol & 0.000026 & Ixc & W & $G, T$ & in situ \\
\hline 547 & 0 & & 0 & & 0 & & Sol & 0.000077 & EAAA & W & $\mathrm{P}, \mathrm{Ti}$ & ex situ \\
\hline 548 & 0 & & 0 & & 0 & & BG & 0.001066 & IXC & W & & \\
\hline 549 & 0.0096 & 0.3611 & 0 & & 0.0161 & 0.4875 & AA, Sol & 0.000384 & IXC & W & $P, T$ & in situ \\
\hline 550 & 0.0132 & & 0 & & 0 & & Sol & 0.000051 & EAAA & $\mathrm{D}$ & $\mathrm{P}, \mathrm{Ti}$ & ex situ \\
\hline 551 & 0 & & 0 & & 0 & & Pal & 0 & EAAA & W & $\mathrm{Ti}$ & ex situ \\
\hline 552 & 0.0263 & & 0 & & 0 & & BG & 0 & Mex & $\mathrm{D}$ & T & ex situ \\
\hline 556 & 0 & & 0 & & 0 & & Bal, BEA, Me, Pal, Palm, Sol, TS & 0.000241 & IXC & $R-W, W$ & $F, G, T, U r$ & in situ \\
\hline 557 & 0 & & 0.0025 & & 0 & & Sol & 0.000077 & $A C$ & $\mathrm{D}$ & P, Prp, Ti & ex situ \\
\hline 558 & 0 & & 0 & & 0 & & $\mathrm{Bal}, \mathrm{BG}$, Sol & 0.000128 & IXC & $R-W$ & $F, G, T, U r$ & in situ \\
\hline 559 & 0 & & 0 & & 0 & -1.218 & $\mathrm{BG}, \mathrm{Me}, \mathrm{TS}$ & 0.001730 & Ixc & W & $F, G, T$ & in situ \\
\hline
\end{tabular}


Table 5 Species, Spanish common names, number of uses, percentage of families that consume it; cognitive prominence values expressed as $\mathrm{S}=$ Sutrop relative prominence index ${ }^{2}$ and biocultural importance expressed as first component value of the principal component analysis by use type (edible, medicinal, firewood, fodder, ceremonial and ornamental; distribution on vegetal types, importance ecological index value (EIVI); specie origin region, ecological status, management practices and management site with respect to species wild populations (Continued)

\begin{tabular}{|c|c|c|c|c|c|c|c|c|c|c|c|}
\hline 560 & 0 & 0 & & 0.0137 & -1.0373 & BEM, Me & 0 & Ixc & W & $F, G$ & in situ \\
\hline 561 & 0 & 0 & & 0 & & BEA & 0 & Ixc & W & G & in situ \\
\hline 562 & 0 & 0 & & 0 & & Sol & 0.000077 & EAAA & W & $\mathrm{P}, \mathrm{Ti}$ & ex situ \\
\hline 563 & 0 & 0 & & 0 & & BEA & 0 & IXc & W & & \\
\hline 564 & 0 & 0 & & 0 & & Bal, Sol & 0.000026 & Ixc & W & $F, G, T, U r$ & in situ \\
\hline 565 & 0 & 0 & & 0 & & Bal, Sol, TS & 0.006657 & Ixc & R-W & $G, T, U r$ & in situ \\
\hline 566 & 0 & 0 & & 0 & & Bal, BEA, Me, Sol & 0.000051 & Ixc & $R-W, W$ & $G, T, U r$ & in situ \\
\hline 567 & 0 & 0 & & 0 & & Bal, Sol & 0.000051 & Ixc & $R-W, W$ & $G, T, U r$ & in situ \\
\hline 568 & 0 & 0 & 0.5508 & 0 & & CaMy & 0 & Ixc & W & $\mathrm{G}, \mathrm{P}, \mathrm{Ti}$ & ex situ, in situ \\
\hline 569 & 0 & 0 & & 0 & & Iz & 0 & Ixc & W & & \\
\hline 571 & 0 & 0 & & 0 & & Me & 0 & Ixc & W & $F, P$ & in situ \\
\hline 572 & 0 & 0 & -1.1913 & 0 & & BEA, Iz, Palm & 0 & Ixc & W & G & in situ \\
\hline 574 & 0 & 0 & 0.4864 & 0 & & $\mathrm{Me}$, Sol & 0 & Ixc & W & $\mathrm{G}, \mathrm{P}, \mathrm{Ti}$ & ex situ, in situ \\
\hline 575 & 0 & 0 & & 0 & & BEA, Pal & 0 & Ixc & W & $\mathrm{G}, \mathrm{P}, \mathrm{Ti}$ & ex situ, in situ \\
\hline 576 & 0 & 0 & & 0 & & BEA & 0 & Ixc & W & $\mathrm{Ti}$ & in situ \\
\hline 577 & 0 & 0 & 1.1964 & 0 & & BEA, BEM, Pal, Sol & 0.000051 & Ixc & W & $\mathrm{G}, \mathrm{P}, \mathrm{Ti}$ & ex situ, in situ \\
\hline 584 & 0 & 0.0333 & 2.2487 & 0 & & BEA, Pal, Sol & 0.000205 & Ixc & W & G, P, Prp, Ti & ex situ, in situ \\
\hline 578 & 0 & 0 & & 0 & & Me & 0 & Ixc & W & $F, P$ & in situ \\
\hline 573 & 0 & 0 & & 0 & & BEA & 0 & Ixc & W & $\mathrm{P}, \mathrm{Ti}$ & ex situ, in situ \\
\hline 579 & 0 & 0.0524 & 1.2962 & 0 & & Pal, Sol, TS & 0.000281 & Ixc & W & P, Prp, Ti & ex situ \\
\hline 580 & 0 & 0.0439 & 1.2722 & 0 & & AA, Pal, Sol & 0.000153 & Ixc & W & P, Prp, Ti & ex situ \\
\hline 581 & 0 & 0 & & 0 & & BEA & 0 & Ixc & W & & \\
\hline 582 & 0 & 0 & & 0 & & BEA & 0 & Ixc & W & $\mathrm{Ti}$ & in situ \\
\hline 570 & 0 & 0 & & 0 & & BEA, Iz & 0 & Ixc & W & & \\
\hline 583 & 0 & 0 & & 0 & & BEA, Pal & 0 & Ixc & W & $\mathrm{P}, \mathrm{Ti}$ & ex situ, in situ \\
\hline 585 & 0 & 0 & & 0 & & BEA & 0 & IXc & W & $\mathrm{Ti}$ & in situ \\
\hline 586 & 0 & 0.0046 & 1.2724 & 0 & & BEA, BEM, Pal, Sol & 0.000026 & Ixc & W & $\mathrm{G}, \mathrm{P}, \mathrm{Ti}$ & ex situ, in situ \\
\hline 587 & 0 & 0 & -1.6375 & 0 & & Iz & 0 & Ixc & W & G & in situ \\
\hline 588 & 0 & 0 & & 0 & & BEA, Me, Pal & 0 & Ixc & W & $\mathrm{P}, \mathrm{Ti}$ & ex situ, in situ \\
\hline 589 & 0 & 0 & & 0 & & BEA, Me, Pal & 0 & Ixc & W & $\mathrm{P}, \mathrm{Ti}$ & ex situ, in situ \\
\hline 590 & 0 & 0 & & 0 & & $\mathrm{NE}$, Sol & 0.000026 & Ixc & W & $\mathrm{P}, \mathrm{Ti}$ & ex situ, in situ \\
\hline
\end{tabular}


Table 5 Species, Spanish common names, number of uses, percentage of families that consume it; cognitive prominence values expressed as $\mathrm{S}=$ Sutrop relative prominence index ${ }^{2}$ and biocultural importance expressed as first component value of the principal component analysis by use type (edible, medicinal, firewood, fodder, ceremonial and ornamental; distribution on vegetal types, importance ecological index value (EIVI); specie origin region, ecological status, management practices and management site with respect to species wild populations (Continued)

\begin{tabular}{|c|c|c|c|c|c|c|c|c|c|c|c|}
\hline 719 & 0 & & 0 & & 0 & Iz & 0 & $\mathrm{IXC}$ & W & & \\
\hline 720 & 0 & & 0 & -1.0665 & 0 & BEA, BN, Me, Palm & 0.003728 & Ixc & $R-W, W$ & $F, G$ & in situ \\
\hline 591 & 0 & & 0 & & 0 & BEA, Pal & 0 & Ixc & W & $\mathrm{F}$ & in situ \\
\hline 722 & 0 & & 0.0389 & -1.1735 & 0 & $\mathrm{BEA}, \mathrm{BEC}, \mathrm{BEM}, \mathrm{Me}$ & 0 & Ixc & $R-W, W$ & G & in situ \\
\hline 723 & 0 & & 0 & -1.4246 & 0 & $\mathrm{Iz}, \mathrm{Pal}, \mathrm{Palm}$ & 0.000396 & Ixc & $R-W, W$ & $G, T$ & in situ \\
\hline 594 & 0 & & 0 & & 0 & $\mathrm{Bal}$, Sol & 0 & Ixc & R-W & $\mathrm{T}$ & in situ \\
\hline 592 & 0 & -0.0837 & 0 & & 0 & $\mathrm{Iz}, \mathrm{Me}$, Sol, TS & 0.038091 & Ixc & W & $F, P, T$ & in situ \\
\hline 593 & 0.0066 & 1.1688 & 0 & & 0 & $\mid z$, Sol & 0.000026 & Ixc & W & $F, G, P, \operatorname{Prp}$ & ex situ, in situ \\
\hline 595 & 0 & -0.7869 & 0 & & 0 & $\mathrm{BEA}, \mathrm{BEC}, \mathrm{BN}, \mathrm{Me}$ & 0.026267 & Ixc & W & $F, G$ & in situ \\
\hline 596 & 0 & & 0 & -0.909 & 0 & Bal, Pal, Sol, TS & 0.001314 & Ixc & R-W & $G, T, U r$ & in situ \\
\hline 597 & 0 & -0.7604 & 0 & & 0 & Sol & 0 & Ixc & W & $G, P, T$ & in situ \\
\hline 598 & 0 & & 0 & & 0 & Sol & 0 & Ixc & W & $G, T$ & in situ \\
\hline 761 & 0 & 1.1156 & 0 & & 0 & $\mathrm{CaCe}$, SB, Sol & 0 & Ixc & W & $\mathrm{G}, \mathrm{P}, \mathrm{Ti}$ & ex situ, in situ \\
\hline 721 & 0 & & 0 & & 0 & Iz & 0 & Ixc & W & $\mathrm{F}$ & in situ \\
\hline 599 & 0 & & 0 & & 0 & Iz & 0 & Ixc & R-W & G & in situ \\
\hline 600 & 0 & & 0 & & 0 & Palm, Sol & 0.000205 & Mex & W & $\mathrm{P}, \mathrm{Ti}$ & ex situ \\
\hline 601 & 0.0697 & 1.3422 & 0 & & 0 & BEM & 0 & Ixc & W & $\mathrm{G}, \mathrm{P}, \mathrm{Ti}$ & ex situ, in situ \\
\hline 602 & 0 & & 0 & & 0 & BEA & 0 & Ixc & W & $\mathrm{F}$ & in situ \\
\hline 603 & 0.0103 & & 0 & & 0 & Pal, Sol & 0.000102 & Mex & W & P, Prp, Ti & ex situ \\
\hline 717 & 0 & & 0 & & 0 & Sol & 0.000051 & EAAA & D & P, Prp & ex situ \\
\hline 718 & 0.0096 & -0.9247 & 0 & & 0 & Paz, VR & 0.000458 & Ixc & W & $F, G$ & in situ \\
\hline 724 & 0 & & 0 & & 0 & Sol & 0.000077 & Ixc & $R-W, W$ & $\mathrm{~T}$ & in situ \\
\hline 725 & 0 & & 0 & -1.4068 & 0 & BEA, Palm & 0.000385 & Ixc & $R-W, W$ & G & in situ \\
\hline 726 & 0 & & 0 & & 0 & BEA & 0 & Ixc & $R-W, W$ & F & in situ \\
\hline 604 & 0 & & 0 & & 0 & $B G, V R$ & 0 & Nat-EAAA & W & & \\
\hline 727 & 0 & & 0 & & 0 & $\mathrm{BEA}, \mathrm{BG}, \mathrm{BN}, \mathrm{Me}$ & 0.003738 & IXc & $R-W, W$ & G & in situ \\
\hline 728 & 0 & & 0 & & 0 & Sol & 0 & EAAA & w & P, Prp & ex situ \\
\hline 729 & 0 & & 0 & & 0 & BEA & 0 & Ixc & W & G & in situ \\
\hline 605 & 0 & & 0 & & 0 & $\mathrm{BG}, \mathrm{Pal}$ & 0 & Ixc & R-W & $\mathrm{T}, \mathrm{Ur}$ & in situ \\
\hline 606 & 0 & & 0 & & 0 & Bal & 0 & Ixc & W & $F, G, T, U r$ & in situ \\
\hline 607 & 0 & & 0 & & 0 & $\mathrm{BN}, \mathrm{Iz}, \mathrm{Me}$ & 0.001851 & Nat-EAAA & W & $F, G$ & ex situ \\
\hline
\end{tabular}


Table 5 Species, Spanish common names, number of uses, percentage of families that consume it; cognitive prominence values expressed as $S=$ Sutrop relative prominence index ${ }^{2}$ and biocultural importance expressed as first component value of the principal component analysis by use type (edible, medicinal, firewood, fodder, ceremonial and ornamental; distribution on vegetal types, importance ecological index value (EIVI); specie origin region, ecological status, management practices and management site with respect to species wild populations (Continued)

\begin{tabular}{|c|c|c|c|c|c|c|c|c|c|}
\hline 608 & 0 & 0 & 0 & BEA & 0 & $\mid x C$ & W & $\mathrm{F}$ & in situ \\
\hline 609 & 0 & 0 & 0 & BEA, BEC, BN, Me, Palm, Paz, TS & 0.059386 & IxC & W & $F, T, U r$ & in situ \\
\hline 610 & 0 & 0 & 0 & BG, Pal, Sol, VR & 0.001636 & Nat-EAAA & W & $F, P, \operatorname{Prp}$ & ex situ \\
\hline 611 & 0 & 0 & 0 & Bal, Sol, TS & 0 & Nat-EAAA & $\mathrm{D}$ & $F, P, \operatorname{Prp}, T, U r$ & ex situ \\
\hline 612 & 0 & 0 & 0 & Bal, Iz, Sol & 0 & Ixc & W & $F, G, T, U r$ & in situ \\
\hline 614 & 0 & 0 & 0 & Bal & 0 & Ixc & W & $F, G, T, U r$ & in situ \\
\hline 615 & 0 & 0 & 0 & Bal & 0 & IXC & W & $F, G, T, U r$ & in situ \\
\hline 613 & 0 & 0 & 0 & Paz & 0.004938 & IXC & W & $\mathrm{F}$ & in situ \\
\hline 616 & 0 & 0 & 0 & Sol & 0.000051 & EAAA & W & P, Prp & ex situ \\
\hline 617 & 0 & 0 & 0 & Bal & 0 & Nat-EAAA & R-W & $\mathrm{F}$ & ex situ \\
\hline 618 & 0 & 0 & 0 & Paz & 0.000709 & Nat-EAAA & R-W & F & ex situ \\
\hline 620 & 0 & 0 & 0 & Paz, TS & 0.005333 & IXC & W & $F, G, T, U r$ & in situ \\
\hline 621 & 0 & 0 & 0 & Bal & 0 & Ixc & W & $F, G, T, U r$ & in situ \\
\hline 619 & 0 & 0 & 0 & Bal & 0 & Ixc & W & $F, G, T, U r$ & in situ \\
\hline 622 & 0 & 0 & 0 & $\mathrm{Iz}$ & 0 & IXC & W & $F$ & in situ \\
\hline 623 & 0 & 0 & 0 & Iz & 0 & Ixc & W & F & in situ \\
\hline 624 & 0 & 0 & 0 & BN, Palm, Paz & 0.002708 & IXC & W & F & in situ \\
\hline 625 & 0 & 0 & 0 & Sol, TS & 0.000026 & EAAA & $D$ & P, Prp, T & ex situ \\
\hline 626 & 0 & 0 & 0 & Me & 0 & IxC & W & & \\
\hline 627 & 0 & 0 & 0 & Paz & 0.003002 & IXC & W & F & in situ \\
\hline 628 & 0 & 0 & 0 & Iz & 0.001189 & IXC & W & G & in situ \\
\hline 629 & 0 & 0 & 0 & BEA, BG & 0.003568 & Ixc & W & G & in situ \\
\hline 630 & 0 & 0 & 0 & $\mathrm{Iz}$ & 0 & Ixc & W & F & in situ \\
\hline 631 & 0 & 0 & 0 & Sol & 0.000026 & EAAA & $\mathrm{D}$ & $E, P$ & ex situ \\
\hline 632 & 0 & 0 & 0 & $\mathrm{Iz}$ & 0 & Ixc & W & G & in situ \\
\hline 638 & 0 & 0 & 0 & Paz & 0 & Nat-EAAA & R-W & $\mathrm{F}$ & ex situ \\
\hline 633 & 0 & 0 & 0 & Sol & 0.000026 & EAAA & $\mathrm{D}$ & P, Prp & ex situ \\
\hline 634 & 0 & 0 & 0 & BEA, BG, Me, Paz & 0.003708 & IXC & W & $F, G$ & in situ \\
\hline 635 & 0 & 0 & 0 & Iz, Palm, Paz & 0.002422 & IXC & W & $F, G$ & in situ \\
\hline 636 & 0 & 0 & 0 & $\mathrm{Me}$ & 0 & IXC & W & $F, G$ & in situ \\
\hline 637 & 0.0344 & 0 & 0 & Sol, TS & 0 & EAAA & $\mathrm{D}$ & P, Prp & ex situ \\
\hline
\end{tabular}


Table 5 Species, Spanish common names, number of uses, percentage of families that consume it; cognitive prominence values expressed as $S=$ Sutrop relative prominence index ${ }^{2}$ and biocultural importance expressed as first component value of the principal component analysis by use type (edible, medicinal, firewood, fodder, ceremonial and ornamental; distribution on vegetal types, importance ecological index value (EIVI); specie origin region, ecological status, management practices and management site with respect to species wild populations (Continued)

\begin{tabular}{|c|c|c|c|c|c|c|c|c|c|}
\hline 639 & 0.0376 & 0 & 0 & Sol, TS & 0.000230 & Mex & $\mathrm{D}$ & $F, P, \operatorname{Prp}, T$ & ex situ \\
\hline 640 & 0 & 0 & 0 & Paz & 0 & Ixc & W & $F, G$ & in situ \\
\hline 641 & 0 & 0 & 0 & Paz & 0.003967 & Ixc & W & $F, G$ & in situ \\
\hline 642 & 0 & 0 & 0 & BEA, BEC & 0.008764 & Ixc & W & F & in situ \\
\hline 643 & 0 & 0 & 0 & Iz & 0.020134 & Ixc & W & $\mathrm{F}$ & in situ \\
\hline 644 & 0 & 0 & 0 & Iz & 0 & Ixc & W & $\mathrm{F}$ & in situ \\
\hline 645 & 0 & 0 & 0 & Iz & 0 & Ixc & W & F & in situ \\
\hline 646 & 0 & 0 & 0 & iz & 0 & Ixc & W & F & in situ \\
\hline 647 & 0 & 0 & 0 & iz & 0 & Ixc & W & $\mathrm{F}$ & in situ \\
\hline 648 & 0 & 0 & 0 & BEA & 0 & Ixc & W & $\mathrm{F}$ & in situ \\
\hline 649 & 0 & 0 & 0 & Bal, Sol & 0 & Ixc & W & $F, G, T, U r$ & in situ \\
\hline 650 & 0 & 0 & 0 & BEA, BN, CaCe, Me, Pal, Palm & 0.011792 & Ixc & W & $F, G$ & in situ \\
\hline 651 & 0 & 0 & 0 & BEA, BEC, Iz & 0.008358 & Ixc & W & & \\
\hline 652 & 0 & 0 & 0 & $\mathrm{BN}, \mathrm{Iz}$ & 0.007838 & Ixc & W & $F, G$ & in situ \\
\hline 653 & 0 & 0 & 0 & Bal, Sol & 0 & Nat-EAAA & W & T & ex situ \\
\hline 654 & 0 & 0 & 0 & BEA, BEM & 0 & Ixc & W & $\mathrm{F}$ & in situ \\
\hline 655 & 0 & 0 & 0 & BEM & 0 & Ixc & W & & \\
\hline 656 & 0 & 0 & 0 & BEA, BEM & 0 & Ixc & W & $\mathrm{F}$ & in situ \\
\hline 658 & 0 & 0 & 0 & Iz & 0.001316 & Ixc & W & & \\
\hline 657 & 0 & 0 & 0 & Me & 0.000872 & Ixc & W & & \\
\hline 660 & 0 & 0 & 0 & BG & 0.003064 & Nat-Uk & W & $\mathrm{F}$ & ex situ \\
\hline 661 & 0.0066 & 0 & 0 & Bal, BN, Iz, Palm, Sol, TS & 0.002474 & Nat-EAAA & R-W & $G, T$ & ex situ \\
\hline 759 & 0 & 0 & 0 & $\mathrm{CaCe}$ & 0 & Ixc & W & & \\
\hline 662 & 0 & 0 & 0 & Sol & 0.000051 & EAAA & W & $\mathrm{P}, \mathrm{Ti}$ & ex situ \\
\hline 663 & 0 & 0 & 0 & Me & 0 & Ixc & W & & \\
\hline 664 & 0 & 0 & 0 & $B G, V R$ & 0.004886 & Ixc & W & G & in situ \\
\hline 665 & 0 & 0 & 0 & BEA & 0 & Ixc & W & & \\
\hline 666 & 0 & 0 & 0 & $\mathrm{Iz}, \mathrm{Me}$ & 0 & Ixc & W & & \\
\hline 667 & 0 & 0 & 0 & VR & 0 & Ixc & W & & \\
\hline 668 & 0 & 0 & 0 & Iz & 0 & Ixc & W & & \\
\hline 671 & 0 & 0 & 0 & BEA, BEM, BG, Pal, VR & 0 & IXc & W & $\mathrm{F}$ & in situ \\
\hline
\end{tabular}


Table 5 Species, Spanish common names, number of uses, percentage of families that consume it; cognitive prominence values expressed as $S=$ Sutrop relative prominence index ${ }^{2}$ and biocultural importance expressed as first component value of the principal component analysis by use type (edible, medicinal, firewood, fodder, ceremonial and ornamental; distribution on vegetal types, importance ecological index value (EIVI); specie origin region, ecological status, management practices and management site with respect to species wild populations (Continued)

\begin{tabular}{|c|c|c|c|c|c|c|c|c|c|c|c|c|}
\hline 672 & 0 & & 0 & & 0 & & $\mathrm{Me}, \mathrm{Paz}$ & 0 & Ixc & W & & \\
\hline 673 & 0 & & 0 & & 0 & & Sol & 0 & EAAA & W & E, P, Prp & ex situ \\
\hline 674 & 0 & & 0 & -1.5677 & 0 & & BEA & 0 & Ixc & W & G & in situ \\
\hline 675 & 0 & & 0 & & 0 & & Iz & 0 & Ixc & W & G & in situ \\
\hline 676 & 0 & -0.8476 & 0 & & 0 & & Pal, Sol & 0.000128 & IXC & W & $\mathrm{G}, \mathrm{T}$ & in situ \\
\hline 677 & 0 & & 0 & & 0 & & $\mathrm{CaCe}$ & 0 & Ixc & W & G & in situ \\
\hline 679 & 0 & & 0 & & 0 & & Me & 0 & Ixc & W & G & in situ \\
\hline 680 & 0.0108 & -0.4619 & 0 & & 0 & & Paz, TS & 0 & IXc & D & G, Prp, T & in situ \\
\hline 681 & 0.0082 & & 0 & & 0 & & Sol & 0.000205 & EAAA & D & E, P, Prp, Ti & ex situ \\
\hline 682 & 0 & & 0.0147 & -1.3761 & 0 & & $\mathrm{Me}, \mathrm{SB}$ & 0 & Ixc & W & G & in situ \\
\hline 678 & 0 & 0.033 & 0 & & 0 & & BEA, BEC, BN, Iz, Me, Palm, TS & 0.045749 & Ixc & W & $F, G, T$ & in situ \\
\hline 686 & 0.0150 & & 0 & & 0 & & Sol & 0.000409 & EAAA & $\mathrm{D}$ & $\mathrm{P}, \mathrm{Ti}$ & ex situ \\
\hline 683 & 0.0095 & & 0 & & 0 & & Sol & 0.000153 & EAAA & $\mathrm{D}$ & P, Prp, Ti & ex situ \\
\hline 684 & 0.0129 & & 0 & & 0 & & Pal, Sol & 0.000358 & EAAA & $\mathrm{D}$ & P, Prp, Ti & ex situ \\
\hline 685 & 0.0029 & & 0 & & 0 & & $\mathrm{Paz}, \mathrm{TS}$ & 0 & TCV & $\mathrm{D}$ & G, Prp, T & ex situ \\
\hline 687 & 0 & & 0.0486 & & 0 & & Sol & 0.000256 & EAAA & $\mathrm{D}$ & P, Prp, Ti & ex situ \\
\hline 688 & 0 & & 0 & & 0 & & Sol & 0.000026 & EAAA & $\mathrm{D}$ & $\mathrm{P}, \mathrm{Ti}$ & ex situ \\
\hline 689 & 0 & & 0 & & 0 & & Me & 0 & Ixc & W & & \\
\hline 690 & 0 & & 0.0058 & -1.6222 & 0 & & Me & 0 & Ixc & W & G & in situ \\
\hline 691 & 0 & & 0 & -0.5674 & 0 & & BEA, BEC, Me, Palm, Paz, TS & 0.001181 & Ixc & W & $F, G, T, U r$ & in situ \\
\hline 692 & 0 & & 0.0661 & 1.7204 & 0 & 0.6818 & $\mathrm{CaCe}, \mathrm{Me}$, Sol & 0.000291 & Ixc & W & $\mathrm{G}, \mathrm{P}, \mathrm{Ti}$ & ex situ, in situ \\
\hline 693 & 0 & & 0 & & 0 & & Iz & 0 & Ixc & W & & \\
\hline 694 & 0 & & 0 & & 0 & & BEA, Me, Palm & 0 & Ixc & W & & \\
\hline 695 & 0 & & 0 & -1.6375 & 0 & & Me, Palm & 0 & Ixc & W & G & in situ \\
\hline 696 & 0 & & 0 & & 0 & & BEA, BEC & 0.005571 & Ixc & W & & \\
\hline 697 & 0 & & 0 & & 0 & & BEA, Me, Pal, Palm & 0.003340 & Ixc & W & G & in situ \\
\hline 698 & 0 & & 0 & & 0 & & BEA, Pal, VR & 0 & Ixc & W & G & in situ \\
\hline 699 & 0 & & 0 & & 0 & & $\mathrm{CaCe}$ & 0 & Ixc & W & & \\
\hline 700 & 0.0132 & & 0 & & 0.0095 & & Sol & 0.000153 & TCV & D & $\mathrm{E}, \mathrm{P}, \operatorname{Prp}, \mathrm{T}$ & ex situ \\
\hline 701 & 0.0124 & & 0 & & 0 & & Sol & 0.000307 & EAAA & D & $\mathrm{E}, \mathrm{P}, \mathrm{T}, \mathrm{Ti}$ & ex situ \\
\hline 704 & 0 & & 0 & & 0 & & Sol & 0.000026 & EAAA & D & $\mathrm{P}, \mathrm{Ti}$ & ex situ \\
\hline
\end{tabular}


Table 5 Species, Spanish common names, number of uses, percentage of families that consume it; cognitive prominence values expressed as $S=$ Sutrop relative prominence index ${ }^{2}$ and biocultural importance expressed as first component value of the principal component analysis by use type (edible, medicinal, firewood, fodder, ceremonial and ornamental; distribution on vegetal types, importance ecological index value (EIVI); specie origin region, ecological status, management practices and management site with respect to species wild populations (Continued)

\begin{tabular}{|c|c|c|c|c|c|c|c|c|c|c|c|}
\hline 703 & 0 & & 0 & 0 & & Sol & 0.000051 & EAAA & $\mathrm{D}$ & $\mathrm{P}, \mathrm{T}, \mathrm{Ti}$ & ex situ \\
\hline 705 & 0 & & 0 & 0 & & Sol & 0.000051 & EAAA & $\mathrm{D}$ & $\mathrm{P}, \mathrm{T}, \mathrm{Ti}$ & ex situ \\
\hline 702 & 0 & & 0 & 0 & & Sol & 0.000026 & EAAA & $\mathrm{D}$ & $\mathrm{P}, \mathrm{Ti}$ & ex situ \\
\hline 706 & 0 & & 0 & 0.0071 & -0.7535 & BEA, BEC, BG, BN, Iz, Me, Palm, Sol, TS & 0.007574 & Ixc & W & $G, T$ & in situ \\
\hline 707 & 0 & & 0 & 0 & & Sol & 0.000230 & EAAA & W & P, Prp & ex situ \\
\hline 708 & 0 & & 0 & 0 & & $\mathrm{I}, \mathrm{SB}$ & 0.000678 & IXC & W & & \\
\hline 709 & 0 & & 0 & 0 & -1.932 & BEA & 0 & IXC & W & G & in situ \\
\hline 710 & 0 & & 0 & 0 & & $\mathrm{CaCe}$ & 0 & IxC & W & G & in situ \\
\hline 394 & 0 & & 0 & 0 & & $\mathrm{Me}$ & 0.000118 & IXC & W & & \\
\hline 711 & 0 & & 0 & 0 & & Pal & 0 & TCV & W & $\mathrm{Ti}$ & ex situ \\
\hline 779 & 0 & & 0 & 0 & & CaMy, BE, Iz & 0 & IXC & W & Ur & in situ \\
\hline 780 & 0 & & 0 & 0 & & Me, Sol & 0.000051 & Ixc & W & Ur & in situ \\
\hline 781 & 0 & & 0 & 0 & & $\mathrm{Iz}, \mathrm{Me}$ & 0 & Ixc & W & G, Ur & in situ \\
\hline 712 & 0 & & 0 & 0.0549 & -0.2043 & BEA, BEC, BN, Iz, Me, Palm, Sol, TS & 0.021155 & IxC & W & $\mathrm{G}, \mathrm{T}$ & in situ \\
\hline 713 & 0 & & 0 & 0 & & SB & 0 & IXC & W & & \\
\hline 715 & 0.0132 & & 0 & 0 & & Sol & 0.000051 & TCV & $\mathrm{D}$ & P, Prp, T & ex situ \\
\hline 716 & 0 & & 0 & 0 & & Iz & 0 & IXC & W & $\mathrm{F}$ & in situ \\
\hline 714 & 0 & & 0 & 0 & & $A A$ & 0 & IXC & W & & \\
\hline 730 & 0 & & 0 & 0 & & BEA, Me & 0 & Ixc & W & G & in situ \\
\hline 731 & 0 & & 0 & 0 & & SB & 0 & IXC & W & & \\
\hline 732 & 0 & & 0 & 0 & & BEA & 0 & IXC & W & & \\
\hline 733 & 0 & & 0.0139 & 0 & & Sol & 0.000051 & $A C$ & D & P, Prp & ex situ \\
\hline 734 & 0.0065 & 4.5368 & 0 & 0 & & SB, Sol & 0.000153 & IXC & $\mathrm{D}, \mathrm{W}$ & $\mathrm{E}, \mathrm{G}, \mathrm{P}, \operatorname{Prp}, \mathrm{T}, \mathrm{Ti}$ & ex situ, in situ \\
\hline 735 & 0.0020 & & 0 & 0 & & Sol & 0.000077 & $A C$ & $\mathrm{D}$ & E, P, Prp, T, Ti & ex situ \\
\hline 736 & 0 & & 0 & 0 & & Me & 0 & Ixc & W & G & in situ \\
\hline 737 & 0 & & 0 & 0 & & BEA, Pal, VR & 0 & IXC & W & $\mathrm{F}$ & in situ \\
\hline 738 & 0 & & 0 & 0 & & Pal & 0 & Ixc & $R-W, W$ & T & in situ \\
\hline 739 & 0 & -1.0133 & 0 & 0 & & Palm, Sol & 0 & IXC & $R-W, W$ & $\mathrm{G}, \mathrm{T}$ & in situ \\
\hline 740 & 0.0020 & -0.9978 & 0 & 0 & & BEA, BG, Pal, Palm, Sol & 0 & IXC & $R-W, W$ & $\mathrm{G}, \mathrm{T}$ & in situ \\
\hline 741 & 0 & & 0 & 0.0069 & & Bal, Pal, Sol, TS & 0.000026 & Nat-AC & $R-W$ & $\mathrm{G}, \mathrm{T}$ & ex situ \\
\hline 742 & 0 & & 0 & 0 & & Sol & 0.000077 & Mex & $\mathrm{D}$ & $\mathrm{G}, \mathrm{T}$ & ex situ \\
\hline
\end{tabular}


Table 5 Species, Spanish common names, number of uses, percentage of families that consume it; cognitive prominence values expressed as $\mathrm{S}=$ Sutrop relative prominence index ${ }^{2}$ and biocultural importance expressed as first component value of the principal component analysis by use type (edible, medicinal, firewood, fodder, ceremonial and ornamental; distribution on vegetal types, importance ecological index value (EIVI); specie origin region, ecological status, management practices and management site with respect to species wild populations (Continued)

\begin{tabular}{|c|c|c|c|c|c|c|c|c|c|c|c|}
\hline 743 & 0.0150 & 0.9152 & 0 & & 0 & Sol, Ts & 0.001383 & Ixc & $D, R-W$ & E, P, Prp, T, Ti & in situ \\
\hline 744 & 0 & & 0 & & 0 & Sol & 0 & $A C$ & $\mathrm{D}$ & $\mathrm{P}, \mathrm{Ti}$ & ex situ \\
\hline 745 & 0 & -1.1768 & 0 & & 0 & Sol & 0.000026 & Ixc & $\mathrm{R}-\mathrm{W}$ & $G, T$ & in situ \\
\hline 746 & 0 & & 0 & & 0 & Sol & 0.000026 & Ixc & $R-W, W$ & $\mathrm{G}, \mathrm{T}$ & in situ \\
\hline 747 & 0 & & 0 & & 0 & BEA, BEC, BG, Palm, Sol & 0.005064 & Ixc & $R-W, W$ & $\mathrm{G}, \mathrm{T}$ & in situ \\
\hline 748 & 0 & -1.3855 & 0 & & 0 & Paz & 0 & Ixc & W & G & in situ \\
\hline 749 & 0.0128 & & 0 & & 0 & Pal, Sol & 0.000205 & TCV & $\mathrm{D}$ & E, P, Prp, T, Ti & ex situ \\
\hline 750 & 0 & & 0 & & 0 & BEA & 0 & Ixc & R-W & G & in situ \\
\hline 751 & 0 & -1.2217 & 0 & & 0 & Sol, BEA, BEC, Me, Pal, Paz & 0 & Ixc & $R-W, W$ & G & in situ \\
\hline 753 & 0 & & 0 & & 0 & $\mathrm{CaCe}$ & 0 & Ixc & $\mathrm{R}-\mathrm{W}$ & & \\
\hline 754 & 0.0044 & & 0 & & 0 & Sol, TS & 0 & $A C$ & $\mathrm{D}$ & P, Prp & ex situ \\
\hline 752 & 0 & & 0 & & 0 & Bal & 0 & Ixc & R-W & $\mathrm{T}$ & in situ \\
\hline 756 & 0 & & 0 & & 0 & $\mathrm{CaCe}$ & 0 & Ixc & W & & \\
\hline 659 & 0 & & 0 & & 0 & BG & 0.001995 & Ixc & W & $\mathrm{F}$ & in situ \\
\hline 757 & 0 & & 0 & & 0 & Palm & 0 & Ixc & W & & \\
\hline 758 & 0 & & 0 & -1.6245 & 0 & BEA, Iz, Pal & 0.000658 & Ixc & W & G & in situ \\
\hline 760 & 0 & & 0.0056 & & 0 & Sol & 0.000077 & Nat-AC & R-W & P, Prp, T & ex situ \\
\hline 762 & 0 & & 0 & & 0 & VR & 0 & Ixc & W & & \\
\hline 764 & 0 & & 0 & & 0 & BEA, Pal, Sol, VR & 0.000026 & Ixc & W & $G, T$ & in situ \\
\hline 765 & 0 & & 0 & & 0 & BEA, Me, Sol & 0.000026 & Ixc & W & P, Prp & ex situ, in situ \\
\hline 766 & 0 & & 0 & & 0 & Sol & 0 & Ixc & W & $\mathrm{G}, \mathrm{T}$ & in situ \\
\hline 768 & 0 & & 0 & & 0 & SB & 0 & Ixc & W & & \\
\hline 769 & 0 & & 0 & & 0 & Palm & 0 & Ixc & W & & \\
\hline 770 & 0 & & 0 & & 0 & Bal, BEA, BN, Sol & 0.001928 & Ixc & $\mathrm{R}-\mathrm{W}$ & G & in situ \\
\hline 771 & 0 & & 0 & & 0 & BEA, BN, Me, Pal, Palm & 0.000747 & Ixc & $R-W, W$ & $F, G, T, U r$ & in situ \\
\hline 772 & 0 & 1.3843 & 0 & & 0 & BEA, BEC, BN, CaCe, Iz, Me, Palm, Sol & 0.003620 & Ixc & $R-W, W$ & $\mathrm{~F}, \mathrm{G}, \mathrm{P}, \mathrm{Ti}$ & ex situ, in situ \\
\hline 773 & 0 & 1.6392 & 0 & & 0 & BEA, BEC, BN, Iz, Me, Pal, Palm, Sol & 0.010387 & Ixc & $R-W, W$ & $\mathrm{G}, \mathrm{T}, \mathrm{Ti}$ & ex situ, in situ \\
\hline 774 & 0 & -0.8419 & 0 & & 0 & $\mathrm{CaCe}, \mathrm{Me}, \mathrm{Pal}$ & 0 & Ixc & W & $F, G$ & in situ \\
\hline 775 & 0.0066 & 1.002 & 0 & & 0 & $\mathrm{Me}$, Sol & 0 & Ixc & W & $\mathrm{G}, \mathrm{P}, \mathrm{Ti}$ & ex situ, in situ \\
\hline 776 & 0 & & 0 & & 0 & BEA & 0 & Ixc & $\mathrm{R}-\mathrm{W}$ & & \\
\hline 777 & 0 & & 0 & & 0 & SB & 0 & IXc & $\mathrm{R}-\mathrm{W}$ & & \\
\hline
\end{tabular}


Table 5 Species, Spanish common names, number of uses, percentage of families that consume it; cognitive prominence values expressed as $S=$ Sutrop relative prominence index ${ }^{2}$ and biocultural importance expressed as first component value of the principal component analysis by use type (edible, medicinal, firewood, fodder, ceremonial and ornamental; distribution on vegetal types, importance ecological index value (EIVI); specie origin region, ecological status, management practices and management site with respect to species wild populations (Continued)

\begin{tabular}{|c|c|c|c|c|c|c|c|c|c|c|}
\hline 778 & 0 & 0 & & 0 & BEA, Sol & 0 & Ixc & $R-W$ & $T$ & in situ \\
\hline 782 & 0 & 0 & & 0 & CaCe, Sol, TS & 0 & $\mid x c$ & R-W & $\mathrm{T}, \mathrm{Ur}$ & in situ \\
\hline 783 & 0 & 0 & & 0 & Sol & 0 & EAAA & D & $\mathrm{P}, \mathrm{Ti}$ & ex situ \\
\hline 27 & 0 & 0 & & 0 & Sol & 0.000205 & EAAA & $D, R-W$ & P, Prp, Ti & ex situ \\
\hline 82 & 0 & 0 & & 0 & BEM, Pz, Sol & 0.000026 & Nat-EAAA & W & $\mathrm{P}, \mathrm{T}, \mathrm{Ti}, \mathrm{Ur}$ & ex situ \\
\hline 83 & 0 & 0 & & 0 & Sol & 0.000102 & EAAA & W & P, Prp & ex situ \\
\hline 784 & 0 & 0 & & 0 & $\mathrm{CaCe}$ & 0 & Ixc & W & & \\
\hline 785 & 0 & 0.025 & -1.433 & 0 & BEM & 0 & Ixc & W & G & in situ \\
\hline
\end{tabular}




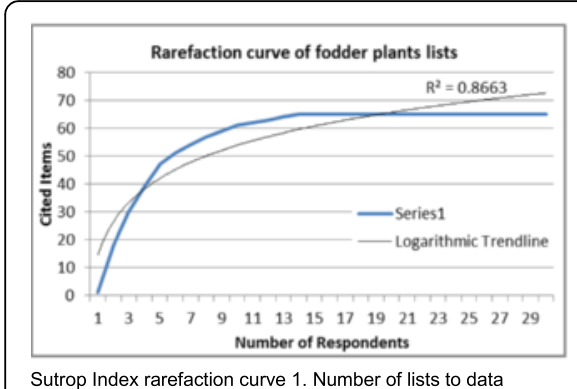

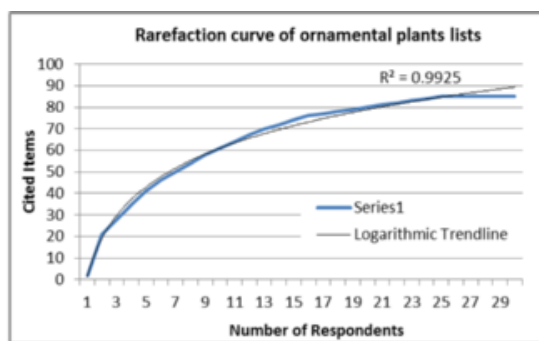

Sutrop Index rarefaction curve 1. Number of list to data saturation for ornamental plants.

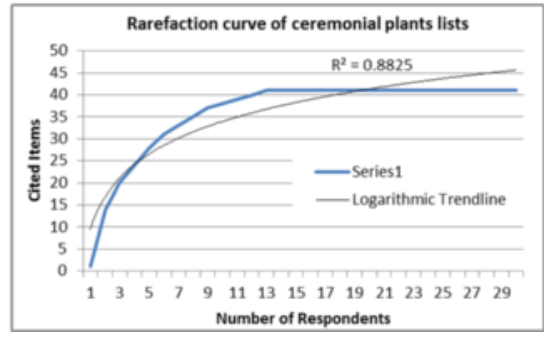

Sutrop Index rarefaction curve 1. Number of list to data saturation for ceremonial plants.

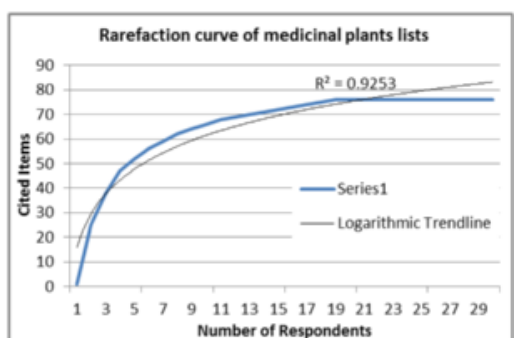

Sutrop Index rarefaction curve 1 . Number of list to data saturation for medicinal plants.

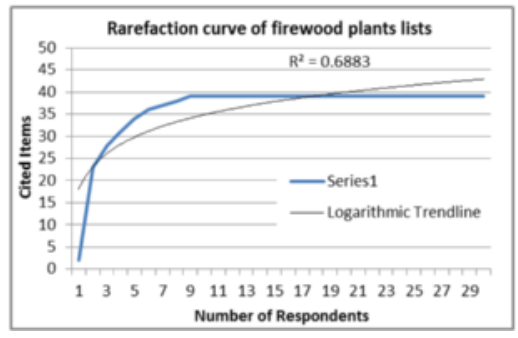

Sutrop Index rarefaction curve 1 . Number of list to data saturation for firewood plants.

Sutrop Index rarefaction cu
saturation for edible plants.

Fig. 6 Rarefaction curves of the Sutrop Index S

Table 6 Santa María Ixcatlán participants and activities in which collaborated

\begin{tabular}{|c|c|c|c|c|c|c|c|c|c|c|c|}
\hline \multicolumn{5}{|c|}{ Participants information } & \multicolumn{4}{|c|}{$\begin{array}{l}\text { Plants common name, use and management practices } \\
\text { documentation }\end{array}$} & \multicolumn{2}{|c|}{$\begin{array}{l}\text { Surveys to estimate } \\
\text { agricultural production } \\
\text { and consumption }\end{array}$} & \multirow[t]{2}{*}{$\begin{array}{l}\text { Free } \\
\text { lists }\end{array}$} \\
\hline ID & Sex & Age & Language & Main activities & $\begin{array}{l}\text { Participants } \\
\text { type }\end{array}$ & $\begin{array}{l}\text { Guide in } \\
\text { trials }\end{array}$ & Homegarden $^{a}$ & $\begin{array}{l}\text { Agricultural } \\
\text { field }^{\mathrm{a}}\end{array}$ & $2000^{\mathrm{a}}$ & $2012^{\mathrm{a}}$ & \\
\hline 1 & Male & 23 & SPA & Mescal production & Key participant & Yes & & & & & Yes \\
\hline 2 & Male & 48 & SPA & $\begin{array}{l}\text { Agriculture, mescal } \\
\text { production }\end{array}$ & Key participant & Yes & & 1 & & 16 & \\
\hline 3 & Male & 70 & SPA, IXC & Agriculture, palm weaver & & & & 6 & 11 & & \\
\hline 4 & Male & 64 & SPA & Agriculture, palm weaver & & & & & & 12 & \\
\hline 5 & Male & 44 & SPA & Agriculture, palm weaver & Key participant & Yes & & 2 & & & Yes \\
\hline 6 & Female & 42 & SPA & $\begin{array}{l}\text { Domestic chores, palm } \\
\text { weaver }\end{array}$ & $\begin{array}{l}\text { Occasional } \\
\text { participant }\end{array}$ & & 8 & & 3 & 15 & \\
\hline 7 & Female & 64 & SPA & $\begin{array}{l}\text { Domestic chores, palm } \\
\text { weaver }\end{array}$ & & & & & 21 & & \\
\hline 8 & Male & & SPA & Agriculture, palm weaver & & & & & 21 & & \\
\hline 9 & Male & & SPA & Agriculture, palm weaver & $\begin{array}{l}\text { Occasional } \\
\text { participant }\end{array}$ & & 5 & & & & \\
\hline 10 & Male & 46 & SPA & Agriculture, commerce & Key participant & & & & & 1 & Yes \\
\hline 11 & Male & 60 & SPA & $\begin{array}{l}\text { Agriculture, mescal } \\
\text { production }\end{array}$ & Key participant & Yes & 10 & & & & \\
\hline 12 & Male & 33 & SPA & $\begin{array}{l}\text { Agriculture, mescal } \\
\text { production, palm weaver }\end{array}$ & & & & & & 13 & \\
\hline 13 & Male & & SPA & Agriculture, palm weaver & $\begin{array}{l}\text { Occasional } \\
\text { participant }\end{array}$ & & & & & & \\
\hline 14 & Male & 65 & SPA & Agriculture, palm weaver & $\begin{array}{l}\text { Occasional } \\
\text { participant }\end{array}$ & & & 3 & & & \\
\hline 15 & Female & 20 & SPA & Palm weaver & $\begin{array}{l}\text { Occasional } \\
\text { participant }\end{array}$ & & & & & & \\
\hline
\end{tabular}


Table 6 Santa María Ixcatlán participants and activities in which collaborated (Continued)

\begin{tabular}{|c|c|c|c|c|c|c|c|c|c|c|c|}
\hline 16 & Male & 71 & SPA & $\begin{array}{l}\text { Agriculture, mescal } \\
\text { production, palm weaver }\end{array}$ & & & & & & & Yes \\
\hline 17 & Female & & SPA & Student & & & & & & & Yes \\
\hline 18 & Female & 58 & SPA & $\begin{array}{l}\text { Domestic chores, palm } \\
\text { weaver }\end{array}$ & $\begin{array}{l}\text { Occasional } \\
\text { participant }\end{array}$ & & 13 & & 2 & & \\
\hline 19 & Female & 33 & SPA & $\begin{array}{l}\text { Domestic chores, palm } \\
\text { weaver }\end{array}$ & & & & & & 1 & \\
\hline 20 & Female & 60 & SPA & $\begin{array}{l}\text { Domestic chores, palm } \\
\text { weaver }\end{array}$ & Key participant & & 15 & & & 7 & \\
\hline 21 & Female & 88 & SPA & $\begin{array}{l}\text { Domestic chores, palm } \\
\text { weaver }\end{array}$ & & & & & 7 & & \\
\hline 22 & Male & & SPA & Agriculture, palm weaver & & & & & 20 & & \\
\hline 23 & Male & 70 & SPA & Agriculture, palm weaver & $\begin{array}{l}\text { Occasional } \\
\text { participant }\end{array}$ & & 3 & & & & Yes \\
\hline 24 & Male & 68 & SPA & Agriculture, palm weaver & & & & & & 14 & \\
\hline 25 & Male & 49 & SPA & $\begin{array}{l}\text { Agriculture, mescal } \\
\text { production, palm weaver }\end{array}$ & & & & & 17 & 5 & \\
\hline 26 & Male & 80 & SPA, IXC & Agriculture, palm weaver & Key participant & Yes & & 4 & & & \\
\hline 27 & Male & & SPA & Agriculture, palm weaver & & & & & & 6 & \\
\hline 28 & Male & 66 & SPA & Agriculture, palm weaver & $\begin{array}{l}\text { Occasional } \\
\text { participant }\end{array}$ & & & & 8 & 4 & \\
\hline 29 & Female & 64 & SPA & $\begin{array}{l}\text { Domestic chores, palm } \\
\text { weaver }\end{array}$ & & & & & 15 & & \\
\hline 30 & Male & 57 & SPA & $\begin{array}{l}\text { Agriculture, mescal } \\
\text { production, palm weaver }\end{array}$ & $\begin{array}{l}\text { Occasional } \\
\text { participant }\end{array}$ & & & & & & Yes \\
\hline 31 & Male & & SPA & Shepherd & Key participant & Yes & & & & & Yes \\
\hline 32 & Male & 97 & SPA, IXC & Palm weaver & Key participant & & & & & & \\
\hline 33 & Female & 75 & SPA & $\begin{array}{l}\text { Domestic chores, palm } \\
\text { weaver }\end{array}$ & & & & & 19 & & \\
\hline 34 & Female & 46 & SPA & $\begin{array}{l}\text { Domestic chores, palm } \\
\text { weaver }\end{array}$ & & & & & 17 & 5 & \\
\hline 35 & Male & & SPA & Student & & & & & & & Yes \\
\hline 36 & Female & 68 & SPA & $\begin{array}{l}\text { Domestic chores, palm } \\
\text { weaver }\end{array}$ & $\begin{array}{l}\text { Occasional } \\
\text { participant }\end{array}$ & & 7 & & & & \\
\hline 37 & Female & & SPA & Commerce, domestic chores & & & & & & & Yes \\
\hline 38 & Female & 16 & SPA & Student & $\begin{array}{l}\text { Occasional } \\
\text { participant }\end{array}$ & & & & & & \\
\hline 39 & Female & & SPA & $\begin{array}{l}\text { Domestic chores, palm } \\
\text { weaver }\end{array}$ & $\begin{array}{l}\text { Occasional } \\
\text { participant }\end{array}$ & & & & & 20 & Yes \\
\hline 40 & Female & 66 & SPA & $\begin{array}{l}\text { Domestic chores, palm } \\
\text { weaver }\end{array}$ & & & & & 10 & & \\
\hline 41 & Female & 32 & SPA & $\begin{array}{l}\text { Domestic chores, palm } \\
\text { weaver }\end{array}$ & & & & & & & Yes \\
\hline 42 & Female & 62 & SPA, IXC & $\begin{array}{l}\text { Domestic chores, palm } \\
\text { weaver }\end{array}$ & Key participant & & 17 & & & 12 & Yes \\
\hline 43 & Male & & SPA & $\begin{array}{l}\text { Agriculture, mescal } \\
\text { production, palm weaver }\end{array}$ & $\begin{array}{l}\text { Occasional } \\
\text { participant }\end{array}$ & Yes & & & & & \\
\hline 44 & Male & & SPA & $\begin{array}{l}\text { Agriculture, construction } \\
\text { worker }\end{array}$ & $\begin{array}{l}\text { Occasional } \\
\text { participant }\end{array}$ & & & & & & \\
\hline 45 & Male & 78 & SPA & Agriculture, palm weaver & & & & & 7 & & \\
\hline 46 & Male & 52 & SPA & Agriculture, palm weaver & & & & & 3 & 15 & \\
\hline
\end{tabular}


Table 6 Santa María Ixcatlán participants and activities in which collaborated (Continued)

\begin{tabular}{|c|c|c|c|c|c|c|c|c|c|c|c|}
\hline 47 & Female & & SPA & $\begin{array}{l}\text { Domestic chores, palm } \\
\text { weaver }\end{array}$ & $\begin{array}{l}\text { Occasional } \\
\text { participant }\end{array}$ & & 10 & & & & \\
\hline 48 & Female & & SPA & $\begin{array}{l}\text { Domestic chores, palm } \\
\text { weaver }\end{array}$ & $\begin{array}{l}\text { Occasional } \\
\text { participant }\end{array}$ & & 12 & & & & \\
\hline 49 & Male & & SPA & Commerce & $\begin{array}{l}\text { Occasional } \\
\text { participant }\end{array}$ & Yes & & & & & \\
\hline 50 & Female & 39 & SPA & Commerce, domestic chores & & & & & & & Yes \\
\hline 51 & Female & 33 & SPA & $\begin{array}{l}\text { Domestic chores, palm } \\
\text { weaver }\end{array}$ & & & & & & 6 & \\
\hline 52 & Male & 74 & SPA, IXC & Agriculture, palm weaver & Key participant & Yes & 16 & & & 9 & Yes \\
\hline 53 & Male & & SPA & Agriculture, palm weaver & $\begin{array}{l}\text { Occasional } \\
\text { participant }\end{array}$ & & 12 & & & & \\
\hline 54 & Female & 43 & SPA & Commerce, domestic chores & $\begin{array}{l}\text { Occasional } \\
\text { participant }\end{array}$ & & & & 6 & & \\
\hline 55 & Male & 30 & SPA & $\begin{array}{l}\text { Agriculture, construction } \\
\text { worker, palm weaver }\end{array}$ & & & & & & & Yes \\
\hline 56 & Female & 73 & SPA & $\begin{array}{l}\text { Domestic chores, palm } \\
\text { weaver }\end{array}$ & & & & & 4 & & \\
\hline 57 & Female & & SPA & $\begin{array}{l}\text { Domestic chores, palm } \\
\text { weaver }\end{array}$ & $\begin{array}{l}\text { Occasional } \\
\text { participant }\end{array}$ & & 9 & & & & \\
\hline 58 & Female & 39 & SPA & $\begin{array}{l}\text { Domestic chores, palm } \\
\text { weaver }\end{array}$ & Key participant & & & & & 16 & Yes \\
\hline 59 & Male & 36 & SPA & Agriculture, palm weaver & & & & & & & Yes \\
\hline 60 & Female & 81 & SPA & $\begin{array}{l}\text { Domestic chores, palm } \\
\text { weaver }\end{array}$ & & & & & & 11 & \\
\hline 61 & Female & 86 & SPA, IXC & $\begin{array}{l}\text { Domestic chores, palm } \\
\text { weaver }\end{array}$ & $\begin{array}{l}\text { Occasional } \\
\text { participant }\end{array}$ & Yes & & & 9 & 2 & \\
\hline 62 & Male & 30 & SPA & Blacksmith & $\begin{array}{l}\text { Occasional } \\
\text { participant }\end{array}$ & & & & & & \\
\hline 63 & Female & 57 & SPA & $\begin{array}{l}\text { Domestic chores, palm } \\
\text { weaver }\end{array}$ & $\begin{array}{l}\text { Occasional } \\
\text { participant }\end{array}$ & & & 7 & 1 & & \\
\hline 64 & Male & & SPA & $\begin{array}{l}\text { Agriculture, mescal } \\
\text { production, palm weaver, } \\
\text { shepherd }\end{array}$ & $\begin{array}{l}\text { Occasional } \\
\text { participant }\end{array}$ & Yes & & & & & \\
\hline 65 & Male & 71 & SPA & Agriculture, palm weaver & & & & & & 18 & \\
\hline 66 & Female & 49 & SPA, IXC & $\begin{array}{l}\text { Domestic chores, palm } \\
\text { weaver }\end{array}$ & Key participant & Yes & & & 9 & 2 & Yes \\
\hline 67 & Male & 18 & SPA & Agriculture, palm weaver & & Yes & & & & & Yes \\
\hline 68 & Male & 59 & SPA & Agriculture, palm weaver & $\begin{array}{l}\text { Occasional } \\
\text { participant }\end{array}$ & & & & & & \\
\hline 69 & Male & & SPA & Student & & & & & & & Yes \\
\hline 70 & Female & 69 & SPA, IXC & $\begin{array}{l}\text { Domestic chores, palm } \\
\text { weaver }\end{array}$ & Key participant & & 4 & & & 18 & Yes \\
\hline 71 & Male & 46 & SPA & Painter & $\begin{array}{l}\text { Occasional } \\
\text { participant }\end{array}$ & & & & & & \\
\hline 72 & Male & 84 & SPA & Agriculture, palm weaver & & & & & & 11 & \\
\hline 73 & Female & 80 & SPA, IXC & $\begin{array}{l}\text { Domestic chores, palm } \\
\text { weaver }\end{array}$ & Key participant & & 5 & & & & \\
\hline 74 & Male & 36 & SPA & $\begin{array}{l}\text { Agriculture, construction } \\
\text { worker, palm weaver }\end{array}$ & & & & & & & Yes \\
\hline 75 & Female & 55 & SPA & $\begin{array}{l}\text { Domestic chores, palm } \\
\text { weaver }\end{array}$ & & & & & 5 & & \\
\hline
\end{tabular}


Table 6 Santa María Ixcatlán participants and activities in which collaborated (Continued)

\begin{tabular}{|c|c|c|c|c|c|c|c|c|c|c|c|}
\hline 76 & Female & 63 & SPA & $\begin{array}{l}\text { Domestic chores, palm } \\
\text { weaver }\end{array}$ & $\begin{array}{l}\text { Occasional } \\
\text { participant }\end{array}$ & & 3 & & & & Yes \\
\hline 77 & Male & 36 & SPA & Agriculture, palm weaver & & & & & & & Yes \\
\hline 78 & Female & 38 & SPA & $\begin{array}{l}\text { Domestic chores, palm } \\
\text { weaver }\end{array}$ & & & & & & 10 & \\
\hline 79 & Male & 57 & SPA & $\begin{array}{l}\text { Agriculture, mescal } \\
\text { production, palm weaver }\end{array}$ & & Yes & & & & 20 & Yes \\
\hline 80 & Male & 68 & SPA & $\begin{array}{l}\text { Agriculture, construction } \\
\text { worker, palm weaver }\end{array}$ & & & & & 2 & & \\
\hline 81 & Female & & SPA & $\begin{array}{l}\text { Domestic chores, palm } \\
\text { weaver }\end{array}$ & & & & & & & Yes \\
\hline 82 & Female & 60 & SPA & $\begin{array}{l}\text { Domestic chores, palm } \\
\text { weaver }\end{array}$ & $\begin{array}{l}\text { Occasional } \\
\text { participant }\end{array}$ & & 2 & & & & \\
\hline 83 & Female & 31 & SPA, IXC & $\begin{array}{l}\text { Domestic chores, palm } \\
\text { weaver }\end{array}$ & & & & & & & Yes \\
\hline 84 & Male & 12 & SPA & Palm weaver, student & Key participant & Yes & 6 & & & & \\
\hline 85 & Male & & SPA & $\begin{array}{l}\text { Mescal production, palm } \\
\text { weaver }\end{array}$ & $\begin{array}{l}\text { Occasional } \\
\text { participant }\end{array}$ & & & & & & \\
\hline 86 & Female & 53 & SPA & Commerce, domestic chores & & & & & 12 & & \\
\hline 87 & Female & & SPA & $\begin{array}{l}\text { Domestic chores, palm } \\
\text { weaver }\end{array}$ & $\begin{array}{l}\text { Occasional } \\
\text { participant }\end{array}$ & & 9 & & & & \\
\hline 88 & Male & 55 & SPA & Agriculture, palm weaver & & & & & & & Yes \\
\hline 89 & Female & 70 & SPA & $\begin{array}{l}\text { Domestic chores, palm } \\
\text { weaver }\end{array}$ & & & & & & & Yes \\
\hline 90 & Male & 24 & SPA & Agriculture, palm weaver & $\begin{array}{l}\text { Occasional } \\
\text { participant }\end{array}$ & & & & & & Yes \\
\hline 91 & Male & 78 & SPA & Agriculture, palm weaver & $\begin{array}{l}\text { Occasional } \\
\text { participant }\end{array}$ & & & & & 19 & Yes \\
\hline 92 & Female & 62 & SPA & $\begin{array}{l}\text { Domestic chores, palm } \\
\text { weaver }\end{array}$ & & & & & 13 & & \\
\hline 93 & Female & 64 & SPA, IXC & $\begin{array}{l}\text { Domestic chores, palm } \\
\text { weaver, shepherdess }\end{array}$ & Key participant & & 1 & & & 17 & Yes \\
\hline 94 & Male & 73 & SPA & Agriculture, palm weaver & $\begin{array}{l}\text { Occasional } \\
\text { participant }\end{array}$ & & & 5 & & & \\
\hline 95 & Male & 62 & SPA & $\begin{array}{l}\text { Agriculture, mescal } \\
\text { production, palm weaver }\end{array}$ & Key participant & & & & & 7 & Yes \\
\hline 96 & Female & 72 & SPA & $\begin{array}{l}\text { Domestic chores, palm } \\
\text { weaver }\end{array}$ & & & & & 18 & & \\
\hline 97 & Female & 77 & SPA, IXC & $\begin{array}{l}\text { Domestic chores, palm } \\
\text { weaver }\end{array}$ & Key participant & & 11 & & & 14 & \\
\hline 98 & Male & 86 & SPA, IXC & Palm weaver & Key participant & & 14 & & 4 & 3 & \\
\hline 99 & Male & & SPA & Baker & $\begin{array}{l}\text { Occasional } \\
\text { participant }\end{array}$ & & & & & & \\
\hline 100 & Female & 82 & SPA, IXC & $\begin{array}{l}\text { Domestic chores, palm } \\
\text { weaver }\end{array}$ & Key participant & & & & 8 & 4 & Yes \\
\hline 101 & Female & 92 & SPA, IXC & $\begin{array}{l}\text { Domestic chores, palm } \\
\text { weaver }\end{array}$ & & & & & 14 & & \\
\hline 102 & Female & 31 & SPA & $\begin{array}{l}\text { Domestic chores, palm } \\
\text { weaver }\end{array}$ & & & & & & 13 & \\
\hline 103 & Male & 23 & SPA & Agriculture, shepherd & Key participant & Yes & 15 & & & & Yes \\
\hline 104 & Female & 37 & SPA & $\begin{array}{l}\text { Domestic chores, palm } \\
\text { weaver }\end{array}$ & $\begin{array}{l}\text { Occasional } \\
\text { participant }\end{array}$ & & 1 & & & & \\
\hline
\end{tabular}


Table 6 Santa María Ixcatlán participants and activities in which collaborated (Continued)

\begin{tabular}{|c|c|c|c|c|c|c|c|c|c|c|}
\hline 105 & Female & & SPA & $\begin{array}{l}\text { Domestic chores, palm } \\
\text { weaver }\end{array}$ & $\begin{array}{l}\text { Occasional } \\
\text { participant }\end{array}$ & & & & & \\
\hline 106 & Male & 53 & SPA & $\begin{array}{l}\text { Agriculture, mescal } \\
\text { production, palm weaver }\end{array}$ & & & & & & Yes \\
\hline 107 & Male & & SPA & Agriculture & & & & & 8 & \\
\hline 108 & Female & 90 & SPA, IXC & $\begin{array}{l}\text { Domestic chores, palm } \\
\text { weaver }\end{array}$ & $\begin{array}{l}\text { Occasional } \\
\text { participant }\end{array}$ & & & & & \\
\hline 109 & Female & 30 & SPA & $\begin{array}{l}\text { Domestic chores, palm } \\
\text { weaver }\end{array}$ & & & & & & Yes \\
\hline 110 & Female & & SPA & Nurse & $\begin{array}{l}\text { Occasional } \\
\text { participant }\end{array}$ & Yes & & & & \\
\hline 111 & Female & 22 & SPA & Nurse assistant & $\begin{array}{l}\text { Occasional } \\
\text { participant }\end{array}$ & Yes & & & & \\
\hline 112 & Female & 18 & SPA & $\begin{array}{l}\text { Domestic chores, palm } \\
\text { weaver }\end{array}$ & & & & 16 & & \\
\hline 113 & Female & 46 & SPA & $\begin{array}{l}\text { Domestic chores, palm } \\
\text { weaver }\end{array}$ & $\begin{array}{l}\text { Occasional } \\
\text { participant }\end{array}$ & Yes & 6 & & & \\
\hline
\end{tabular}

Note

${ }^{a}$ The data provided make reference to the assigned number to the homegarden and agricultural field, since interview could be made to 1 or more household integrants. Is the same case for surveys in which one or two of the householders could provide information about productive activities and consumption of vegetal resources by the household

\section{Names of Botanical experts who contributed to determine the voucher specimens}

Aarón Rodríguez Contreras, Abisaí Josué GarcíaMendoza, Alfonso Valiente-Banuet, Ana Rosa Lopez Ferrari, Mario Adolfo Espejo Serna, Anna Paizanni Guillén, Claudio Delgadillo Moya, Darisol Pacheco Rivera, Eduardo Ruíz-Sánchez, Emmanuel Pérez Calix, Ernesto Velázquez, Montes, Gerardo A. Salazar Chávez, Guadalupe Cornejo-Tenorio, Guillermo Ibarra Manríquez, José Luis Villaseñor Ríos, Juan Ismael Calzada, Luz María GonzálezVillarreal, María de los Angeles Herrera, Mauricio Antonio Mora Jarvio, Oswaldo Tellez Valdés, Pablo Carrillo-Reyes, Rafael Lira Saade, Rosario Redonda-Martínez, Rosalinda Medina-Lemos, Salvador Arias, Sergio Zamudio Ruíz, Susana Valencia Ávalos, Verónica Juárez-Jaimes, Victor W. Steinmann

\section{Abbreviations}

TEK, traditional ecological knowledge; UNAM, Universidad Nacional Autónoma de México; USA, United States of America

\section{Acknowledgements}

We deeply thank people of Santa María Ixcatlán, the authorities and people who collaborated and gave us their hospitality. We also thank Luz E. García and Emanuel E. González for field work assistance, and Sandra Smith, Michael Swanton, Nicholas Johnson and Denis Costaouec for their support and collaboration in the Ixcatec Ethnobiology Project. We thank the botanical experts of MEXU, PATZ, IZTA and UDG herbaria (Appendix), for their help in identifying the voucher specimens, especially Rosalinda Medina-Lemos and Ismael Calzada, and to three anonymous referees for their suggestions for improving this manuscript.

\section{Funding}

The authors thank the Posgrado en Ciencias Biológicas at the Universidad Nacional Autónoma de México (UNAM) and the Consejo Nacional de Ciencia y Tecnología (CONACYT, Mexico) for supporting PhD studies and a grant for the first author. We also thank financial support for field work from CONACYT (Project CB CB-2013-01-221800), the PAPIIT, UNAM (Research project IN209214), Fundación Alfredo Harp Helú Oaxaca and Fundación UNAM (project IE-282.311.190).

Availability of data and materials

Data that support the analysis and additional data are provided in Appendix.

\section{Authors' contributions}

SRL main author, involved in the study design, field work, analysis of data, wrote the first draft and concluded the final version of this paper. AC main coordinator-supervisor of the research project; participated in data analyses and reviewed several drafts of the manuscript. ERL, MVR and RLF contributed to field work and reviewed final drafts of the manuscript. ITG contributed to data analyses and reviewed the final drafts of the manuscript. All authors read and approved the final manuscript.

\section{Authors' information}

SRL postgraduate student at the Instituto de Investigaciones en Ecosistemas y Sustentabilidad (IIES), UNAM. AC full-time researcher at IIES, UNAM. ERL Master in Sciences student at the Centro de Investigaciones en Geografía Ambiental (CIGA), UNAM. ITG \& MVR posdoctoral at IIES \& CIGA, UNAM. RLF technician of Pronatura México A.C.

\section{Competing interests}

The authors declare that they have no competing interests.

\section{Consent for publication}

Not applicable.

\section{Ethics approval and consent to participate}

Permits for conducting our investigation was obtained in the two phases of field work, with Federal agencies (SEMARNAT and Tehuacán-Cuicatlán Biosphere Reserve-CONANP), local authorities (municipal and land tenure) and Communitarian Assembly to realize the investigation and collect voucher plants in communal lands. Prior oral informed consent was obtained from all participants to realize the interview, survey, free lists and audio-visual recording or visit and gather plants in their homegardens or agricultural fields. Reports of activities and preliminary investigation outcomes have been doing via oral and written reports to the authorities and public presentations to the community of Ixcatlán. 


\section{Author details}

Instituto de Investigaciones en Ecosistemas y Sustentabilidad, UNAM Antigua Carretera a Pátzcuaro 8711, Morelia, Michoacán 58190, Mexico. ${ }^{2}$ Posgrado en Ciencias Biológicas, UNAM, Ciudad Universitaria Del. Coyoacan, C. P. 04510 México, Mexico. ${ }^{3}$ Centro de Investigaciones en Geografía Ambiental, UNAM, Antigua Carretera a Pátzcuaro 8711, Morelia, Michoacán 58190, Mexico.

\section{Received: 1 March 2016 Accepted: 2 July 2016}

Published online: 20 July 2016

\section{References}

1. Toledo VM, Ortiz-Espejel B, Cortés L, Moguel P, de Ordonez M. The multiple use of tropical forests by indigenous peoples in Mexico: a case of adaptive management. Conserv Ecol. 2003;7:9.

2. Berkes F, Folke $C$, editors. Linking social and ecological systems: managment practices and social mechanisms for building resilience. Cambridge: Cambridge University Press; 1998

3. Boege E. El patrimonio biocultural de los pueblos indígenas de México. México: Instituto Nacional de Antropología e Historia and Comisión Nacional para el Desarrollo de los Pueblos Indígenas; 2008.

4. Toledo VM, Barrera-Bassols N. La Memoria Biocultural: la importancia ecológica de las sabidurias tradicionales. Barcelona: Icaria Editorial; 2008.

5. Toledo VM, Boege E, Barrera-Bassols N. The biocultural heritage of Mexico: an overview. Langscape. 2010;2:8-13.

6. Casas A, Camou-Guerrero A, Otero-Arnaiz A, Rangel-Landa S, Cruse-Sanders J, Solís L, et al. Manejo tradicional de biodiversidad y ecosistemas en Mesoamérica: el Valle de Tehuacán. Investig Ambient Cienc y política pública. 2014;6:23-44.

7. Berkes F, Colding J, Folke C. Rediscovery of traditional ecological knowledge as adaptive management. Ecol Appl. 2000;10:1251-62.

8. Moreno-Calles Al, Toledo VM, Casas A. Los sistemas agroforestales tradicionales de México: una aproximación biocultural. Bot Sci. 2013;91:375-98.

9. Pretty J, Adams B, Berkes F, de Athayde SF, Dudley N, Hunn E, et al. The intersections of biological diversity and cultural diversity: Towards. Conserv Soc. 2009;7:100-12.

10. Casas A, Otero-Arnaiz A, Pérez-Negrón E, Valiente-Banuet A. In situ management and domestication of plants in Mesoamerica. Ann Bot. 2007;100:1101-15.

11. Blancas J, Casas A, Rangel-Landa S, Moreno-Calles A, Torres I, Pérez-Negrón E, et al. Plant management in the Tehuacan-Cuicatlan Valley, Mexico. Econ Bot. 2010;64:287-302

12. El OE. gobierno de los bienes comunes. La evolución de las instituciones de acción colectiva. 2nd ed. México: FCE, UNAM, IIS; 2011.

13. Toledo VM. Etnoecology: A conceptual framework for the study of indigenous knowledge of nature. In: Steep JR, editor. Ethnobiology and cultural diversity. USA: International Society of Ethnobiology; 2002. p. 511-22.

14. González-Insuasti MS, Martorell C, Caballero J. Factors that influence the intensity of non-agricultural management of plant resources. Agrofor Syst. 2008;74:1-15.

15. Blancas J, Casas A, Pérez-Salicrup D, Caballero J, Vega E. Ecological and socio-cultural factors influencing plant management in Náhuatl communities of the Tehuacán Valley, Mexico. J Ethnobiol Ethnomed. 2013;9:39.

16. Arellanes $\mathrm{Y}$, Casas A, Arellanes A, Vega E, Blancas J, Vallejo M, et al. Influence of traditional markets on plant management in the Tehuacán Valley. J Ethnobiol Ethnomed. 2013;9:38.

17. Blancas J, Pérez-Salicrup D, Casas A. Evaluando la incertidumbre en la disponibilidad de recursos vegetales. Gaia Sci. 2014;8:137-60.

18. Phillips O, Gentry $\mathrm{AH}$. The useful plants of Tambopata, Peru: II. Additional hypothesis testing in quantitative ethnobotany. Econ Bot. 1993;47:33-43.

19. Albuquerque UP, Soldati GT, Ramos MA, Melo JG, Medeiros PM, Nascimento ALB, et al. The influence of the environment on natural resource use: evidence of apparency. In: Albuquerque UP, Medeiros PM, Casas A, editors. Evolutionary ethnobiology. Switzerland: Springer; 2015. p. $131-47$.

20. Zent EL. Jotï ecogony, Venezuelan Amazon. Environ Res Lett. 2013:8:1-15.

21. Moreno-Calles A, Casas A, Blancas J, Torres I, Masera O. Javier Caballero, et al. Agroforestry systems and biodiversity conservation in arid zones: the case of the Tehuacán Valley, Central México. Agrofor Syst. 2010;80:315-31.

22. Vallejo M, Casas A, Blancas J, Moreno-Calles Al, Solís L, Rangel-Landa S, et al. Agroforestry systems in the highlands of the Tehuacán Valley,
Mexico: indigenous cultures and biodiversity conservation. Agrofor Syst. 2014:88:125-40.

23. MacNeish R. The origins of agriculture and setteled life. Oklahoma: University of Oklahoma Press; 1992.

24. Dávila P, Arizmendi M del C, Valiente-Banuet A, Villaseñor JL, Casas A, Lira R. Biological diversity in the Tehuacán-Cuicatlán Valley, Mexico. Biodivers Conserv. 2002;11:421-42.

25. Lira R, Casas A, Rosas-López R, Paredes-Flores M, Pérez-Negrón E, Rangel-Landa S, et al. Traditional knowledge and useful plant richness in the Tehuacán-Cuicatlán Valley, Mexico. Econ Bot. 2009;63:271-87.

26. Diario Oficial de la Federación. Resolución sobre conflicto por límites de bienes comunales al poblado de Santa María Ixcatlán, municipio del mismo nombre, Estado de Oaxaca. 1948. http://www.dof.gob.mx/. Accessed on 25 May 2015

27. Hironymous MO. Santa María Ixcatlan, Oaxaca: From colonial cacicazgo to modern municipio, Ph.D. thesis. Austin: University of Texas; 2007.

28. Servicio Meteorológico Nacional. Normales climatológicas 1951-2010: Estación 00020129 Santa María Ixcatlán, Oaxaca. 2010. http://www.smn. conagua.gob.mx. Accessed on 25 May 2015.

29. García E, García EC. Climas, Catálogo de metadatos geográficos 1:1000000. 2008. http://www.conabio.gob.mx. Accessed on 25 May 2015.

30. INEGI. Conjunto de datos vectoriales de la carta de uso del suelo y vegetación 1:250,000, serie V (Conjunto Nacional). 2013. www.inegi.org.mx. Accessed on 25 May 2015.

31. INEGI. México en cifras: Santa María Ixcatlán, Oaxaca. 2010. www.inegi.org.mx. Accessed on 25th May 2015.

32. Nava C, Romero M. Ixcatecos, pueblos indígenas del México contemporáneo. México: Comisión Nacional para el Desarrollo de los Pueblos Indígenas; 2007

33. Cook SF. Santa María Ixcatlán: habitat, population, subsistence. Ibero-Amer. Berkeley and Los Angeles: University of California Press; 1958.

34. Lewis MP, Simons GF, Fennig CD, Paul LM, Simons GF, Fennig CD. Ethnologue: Languages of the World. Eighteenthth ed. Dallas: SIL International; 2016. Online version: http://www.ethnologue.com. Accessed on Mar 2016

35. Swanton M. La escritura indígena como "material lingüístico". Una carta en lengua ixcateca al presidente Lázaro Cárdenas. In: Van Doesburg S, editor. Pictografía y escritura alfabética en Oaxaca. Oaxaca: Instituto Estatal de Educación Publica de Oaxaca; 2008. p. 353-87.

36. Valiente-Banuet A, Solís L, Dávila P, del Arizmendi M, Arizmendi Mdel C, Silva C, Ortega-Ramírez J, et al. Guía de la vegetación del Valle de Tehuacán-Cuicatlán. México: Universidad Nacional Autónoma de México, Comisión Nacional para el Conocimiento y Uso de la Biodiversidad, Instituto Nacional de Antropología e Historia, Universidad Autónoma de Tamaulipas, Fundación para la Reserva de la Biosfera Tehuacán-Cuicatlán; 2009.

37. The Plant List Version 1.1. Published on the Internet. 2013. http://www. theplantlist.org/. Accessed on Mar 2016.

38. Martin G. Ethnobotany a methods manual. London and Sterling Earthscan; 2004

39. Pérez-Negrón E, Casas A. Use, extraction rates and spatial availability of plant resources in the Tehuacán-Cuicatlán Valley, Mexico: The case of Santiago Quiotepec, Oaxaca. J Arid Environ. 2007;70:356-79.

40. Delgado-Lemus A, Torres I, Blancas J, Casas A. Vulnerability and risk management of Agave species in the Tehuacán Valley, México. J Ethnobiol Ethnomed. 2014;10:53.

41. Husson F, Lê S, Pagès J. Exploratory multivariate analysis by example using R. United States of America: Chapman \& Hall/CRC; 2011.

42. SAS-Institute-INC. JMP 8.0. 2008. http://www.jmp.com

43. Sutrop U. List task and a cognitive salience index. Field Methods. 2001:13:263-76.

44. Pennec F, Wencelius J, Garine E, Raimond C, Bohbot H. FLAME v1.0: Free-list analysis under Microsoft Excel. 2012.

45. Curtis JT. The vegetation of Wisconsin, an ordination of plant communities. Madison: University of Wisconsin Press; 1959.

46. Casas A, Viveros JL, Caballero J. Etnobotánica mixteca: sociedad, cultura y recursos naturales en la Montaña de Guerrero. México: CONACULTA e Instituto Nacional Indigenista; 1994

47. Farfán B, Casas A, Ibarra-Manríquez G, Pérez-Negrón E. Mazahua ethnobotany and subsistence in the Monarch Butterfly Biosphere Reserve, Mexico. Econ Bot. 2007;61:173-91.

48. Alcorn JB. Huastec Mayan Ethnobotany. Austin: University of Texas Press; 1984. 
49. Hunn ES. A Zapotec natural history. United States of America: The University of Arizona Press; 2008.

50. Moreno-Calles Al, Casas A, García-Frapolli E, Torres-García I. Traditional agroforestry systems of multi-crop "milpa" and "chichipera" cactus forest in the arid Tehuacán Valley, Mexico: their management and role in people's subsistence. Agrofor Syst. 2012;84:207-26.

51. Velázquez DeLara G. Relación de Ixcatlán, Quiotepec y Tecomahuaca. In: Acuña R, editor. Relaciones Geográficas del siglo XVI: Antequera, vol. I. México: Instituto de Investigaciones Antropológicas, UNAM; 1984. p. 223-41.

52. Rangel-Landa S, Rivera-Lozoya E, Casas A. Uso y manejo de las palmas Brahea spp. (Arecaceae) por el pueblo ixcateco de Santa María Ixcatlán Oaxaca, México. Gaia Sci. 2014;8:62-78.

53. García-Frapolli E, Toledo VM, Martínez-Alier J. Apropiación de la naturaleza por una comunidad Maya Yucateca: Un análisis económico-ecológico. Rev. Iberoaméricana Econ. Ecológica. 2008;7:27-42.

54. Toledo VM, Barrera-Bassols N, García-Frapolli E, Alarcón-Cháires P. Uso múltiple y biodiversidad entre los mayas yucatecos (México). Interciencia. 2008:33:345-52.

55. Belcher B, Ruíz-Pérez M, Achdiawan R. Global patterns and trends in the use and management of commercial NTFPs: Implications for livelihoods and conservation. World Dev. 2005;33:1435-52.

56. Casas A, Valiente-Banuet A, Viveros JL, Caballero J, Cortés L, Dávila P, et al. Plant resources of the Tehuacán-Cuicatlán Valley, Mexico. Econ Bot. 2001;55:129-66.

57. Casas A, Rangel-Landa S, Torres I, Pérez-Negrón E, Solís L, Parra F, et al. In situ management and conservation of plant resources in the TehuacanCuicatlan Valley, Mexico: an ethnobotanical and ecological perspective. In: de Albuquerque UP, Alves M, editors. Current topics in Ethnobotany. Kerala: Research Signpost; 2008. p. 1-23.

58. Casas A, Blancas J, Otero-Arnaiz A, Cruse-Sanders J, Lira R, Avendaño A, et al. Evolutionary ethnobotanical studies of incipient domestication of plants in Mesoamerica. In: Lira R, Casas A, Blancas J, editors. Ethnobotany of Mexico: interactions of people and plants in Mesoamerica. New York: Springer; 2016. p. $257-85$.

59. Halstead P, O'Shea J. Bad year economics: cultural responses to risk and uncertainty. Cambridge: Cambridge University Press; 1989.

60. Parlee B, Berkes F. Indigenous knowledge of ecological variability and commons management: a case study on berry harvesting from Northern Canada. Hum Ecol. 2006;34:515-28.

61. Casas A, Parra F, Rangel S, Guillén S, Blancas J, Fiqueredo CJ. Evolutionary ecology and ethnobiology. In: Albuquerque UP, Medeiros PM, Casas A, editors. Evolutionary ethnobiology. Switzerland: Springer; 2015. p. 37-57.

62. Atangana A, Khasa D, Chang S, Degrande A. Tropical agroforestry. Dordrecht: Springer; 2014.

63. Nair PKR. Classification of agroforestry systems. Agrofor Syst. 1985;3:97-128.

64. Moreno-Calles Al, Galicia-Luna VJ, Casas A, Toledo VM, Ramos MV, Santos-Fita D, et al. La Etnoagroforestería: el estudio de los sistemas agroforestales tradicionales de México. Etnobiología. 2014;12:1-16.

65. Casas A, Parra F, Blancas J. Evolution of humans and by humans. In: Albuquerque UP, Medeiros PM, Casas A, editors. Evolutionary ethnobiology. Switzerland: Springer; 2015. p. 21-36.

66. Mendoza E. Los eternos tejedores de Santa María Ixcatlán. México Desconoc. 1998;257:20-5.

67. Bartolomé M. Historia Ixcateca. México: Instituto Nacional de Antropología e Historia, CIESAS unidad Oaxaca and Gobierno del Estado de Oaxaca. 1991.

68. González-Insuasti MS, Caballero J. Managing plant resources: How intensive can it be? Hum Ecol. 2007:35:303-14

69. Paredes M, Lira R, Dávila P. Estudio etnobotánico de Zapotitlán Salinas, Puebla. Acta Botánica Mex. 2007;79:13-61.

70. Larios C, Casas A, Vallejo M, Moreno-Calles Al, Blancas J. Plant management and biodiversity conservation in Náhuatl homegardens of the Tehuacán Valley, Mexico. J Ethnobiol Ethnomed. 2013;9:74.

71. Blanckaert I, Swennen R, Paredes-Flores M, Rosas López R, Lira R. Floristic composition, plant uses and management practices in homegardens of San Rafael Coxcatlán, Valley of Tehuacán-Cuicatlán, Mexico. J Arid Environ. 2004;57:179-202.

72. Castaneda H, Stepp JR. Ethnoecological importance value (EIV) methodology: Assessing the cultural importance of ecosystems as sources of useful plants for the Guaymi people of Costa Rica [Internet]. Ethnobot Res Appl. 2007;5:249-57.
73. Challenger A, Bocco G, Equihua M, Chavero EL, Maass M. La aplicación del concepto del sistema socio-ecológico: alcances, posibilidades y limitaciones en la gestión ambiental de México. Investig Ambient Cienc y política pública. 2014;6:1-21.

74. Lucena RFP, Medeiros PM, de Araújo L E, Alves AGC, Albuquerque UP. The ecological apparency hypothesis and the importance of useful plants in rural communities from northeastern Brazil: an assessment based on use value. J Environ Manage. 2012;96:106-15.

75. Maldonado B, Caballero J, Delgado-Salinas A, Lira R. Relationship between use value and ecological importance of floristic resources of seasonally dry tropical forest in the Balsas river basin, Mexico. Econ Bot. 2013;67:17-29.

76. Medeiros PM, Ladio AH, Albuquerque UP. Local criteria for medicinal plant selection. In: Albuquerque UP, Medeiros PM, Casas A, editors. Evolutionary ethnobiology. Switzerland: Springer; 2015. p. 149-62.

77. Camou-Guerrero A. Los recursos vegetales en una comunidad rarámuri: aspectos culturales, económicos y ecológicos, Ph.D. thesis. Mexico: Universidad Nacional Autónoma de México; 2008.

78. González-Insuasti MS, Casas A, Méndez-Ramírez I, Martorell C, Caballero J. Intra-cultural differences in the importance of plant resources and their impact on management intensification in the Tehuacán Valley, Mexico. Hum Ecol. 2011;39:191-202.

79. Torres I, Blancas J, León A, Casas A. TEK, local perceptions of risk, and diversity of management practices of Agave inaequidens in Michoacán, Mexico. J Ethnobiol Ethnomed. 2015;11:1-20.

80. Berkes F, Turner NJ. Knowledge, learning and the evolution of conservation practice for social-ecological. Hum Ecol. 2006;34:4709-494.

81. Berkes F. Understanding uncertainty and reducing vulnerability: lessons from resilience thinking. Nat Hazards. 2007;41:283-95.

82. Espinosa-García FJ, Díaz-Pérez R. El uso campesino de plantas arvenses como forraje en el Valle de México. Etnoecológica. 1996;3:83-94.

83. Bhagwat $\mathrm{S}$, Willis KJ, Birks HJB, Whittaker RJ. Agroforestry: a refuge for tropical biodiversity? Trends Ecol Evol. 2008;23:261-7.

84. García LE. Aspectos socio-ecológicos para el manejo sustentable del copal en el Ejido de Acateyahualco, Gro, Bachelor thesis. Licenciatura en Ciencias Ambientales, México: Universidad Nacional Autónoma de México; 2012.

85. Fowler CS, Lepofsky D. Traditional resource and environmental management. In: Anderson EN, Pearsall DM, Hunn ES, Turner NJ, editors. Ethnobiology. New Jersey: Wiley-Blackwell; 2011. p. 285-304.

\section{Submit your next manuscript to BioMed Central and we will help you at every step:}

- We accept pre-submission inquiries

- Our selector tool helps you to find the most relevant journal

- We provide round the clock customer support

- Convenient online submission

- Thorough peer review

- Inclusion in PubMed and all major indexing services

- Maximum visibility for your research

Submit your manuscript at www.biomedcentral.com/submit
Ciomed Central 